\title{
A STUDY OF THE POTENTIAL FOR DIGITAL PLATFORMS \\ TO ACHIEVE DESIGN COLLABORATION BETWEEN HOMEBUYERS IN THE DEVELOPMENT PHASE OF MULTI-USER-ORIENTED HOUSING
}

\author{
by \\ Lo Tian Tian \\ B.Arch (NUS), BA Hons (NUS), M.Arch (NUS)
}

\begin{abstract}
A full thesis
submitted to the Victoria University of Wellington in fulfilment of the requirements for the degree of Doctor of Philosophy

In

Architecture

Primary Supervisor: Professor Marc Aurel Schnabel Secondary Supervisor: Tane Moleta
\end{abstract}

Wellington, New Zealand, 2018

(c) Lo Tian Tian 2018 



\section{Abstract}

Urban development and densification are increasing rapidly; this fact has been globally reported. According to a 2014 United Nations report, the world population will increase by $25 \%$ in the next three decades. This significant growth means urban density will also increase drastically, creating an increase in high-rise apartment living quarters to cater for the population growth. Subsequently, the development of housing has been advancing - especially around construction techniques which are becoming more efficient to meet the demand of fast-growing urban populations.

This thesis proposes that simply supplying housing is no longer sufficient to address the requirements of citizens. Denser living environments result in increased dissatisfaction, especially among those living in high-density housing. This research looks specifically into enabling homebuyers to voice their needs and design their living space. In this context, the social paradigm of high-density housing has not progressed much. There is still more a notion of supplying the needed quantity and homebuyers accepting the housing without question. Homebuyers, the main users of the housing, are often absent from both the planning and design process. Recent studies have shown that participation in their community is one of the key themes towards social sustainability. Many public participatory projects and platforms only allow participation in large scale urban developments and planning processes. There is a significant lack of initiatives that include homebuyers in the context of high-rise, high-density housing.

The aim of this research is to explore how a computational tool within a virtual environment can facilitate and support design collaboration and interactions - not only between architects and homebuyers, but among individual and collective homebuyers too. The methodology of the research includes using focus groups to examine how digital tools can support and contribute to the collaborative design process of high-rise, high-density housing. The study is then tested with the public to determine if such design tools facilitate and support design collaborations. 
Three studies were undertaken: The first is a pre-tool development study. It uses a focus group to understand the factors necessary to engage homebuyers, and those factors that hinder such process. A digital tool for collaboration was then developed, based on analysis of the focus group results. A second study determines if the factors identified are sufficient for design collaboration within a digital environment. Based on this analysis, the tool was enhanced and integrated with third party visualisation software to enable the desired digital collaboration. A final study involves the public, examining whether such a design tool facilitates and supports their design collaborations.

Throughout the research development, gamification techniques were introduced and adopted to further explore driving factors and to enhance design interactions. The target audience of this research is homebuyers, who are laypersons in architectural design processes and techniques. Gamification is, therefore, an effective technique to simplify the design process and enable homebuyers to immerse themselves in a collaborative design process. Virtual Reality is used at the final stage to immerse homebuyers further into the design environment and give them clearer feedback about their design decisions.

The findings of the research confirm the benefits this novel collaborative design process has on the overall outcome of high-rise, high-dense buildings. It demonstrates how a virtual design tool can influence the process of consultation and procurement for homebuyers. A metadesign framework has been developed to provide a guide to the decision-making support necessary for such a collaborative design process. Finally, the research explains how such an enhanced communicative design operation can achieve the kind of synergies that break out of the current housing paradigm and take a major step forward in urban development. 


\section{Content}

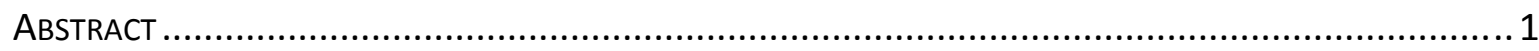

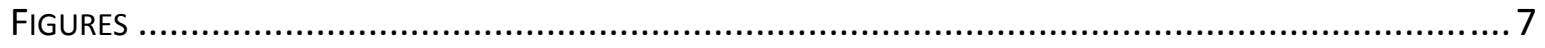

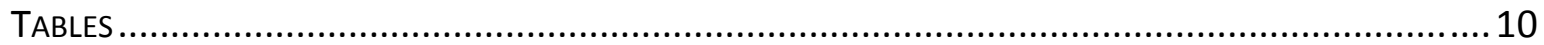

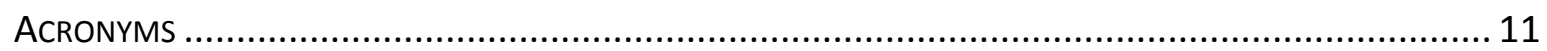

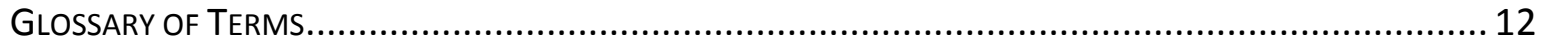

Prelude: Research Background \& Personal Motivation....................................................... 13

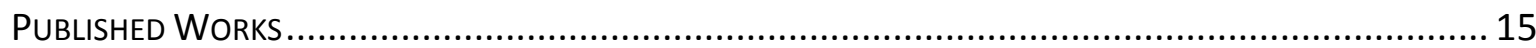

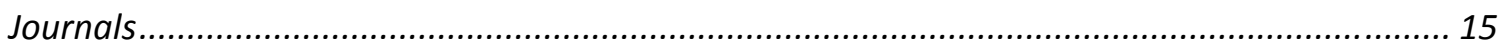

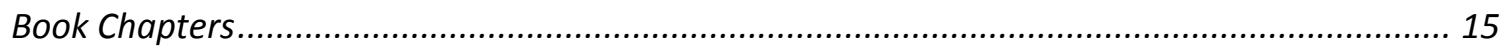

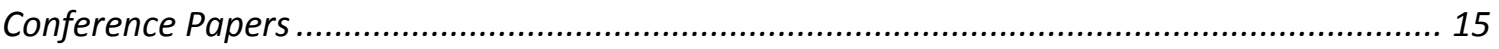

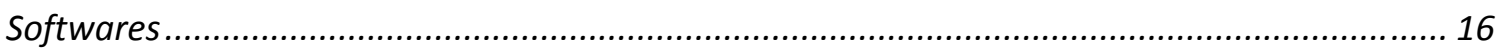

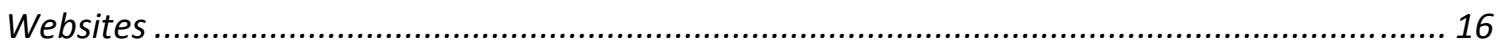

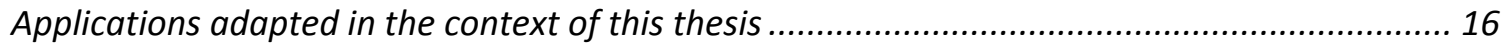

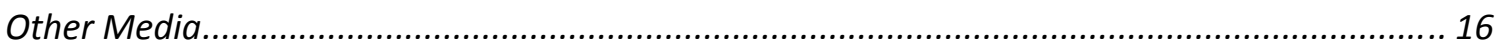

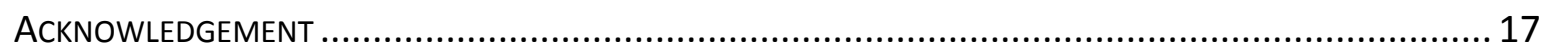

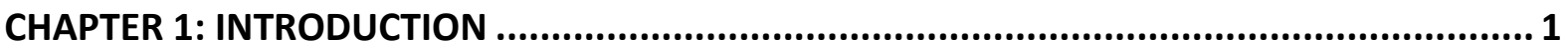

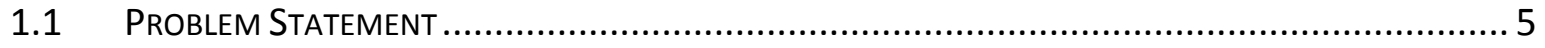

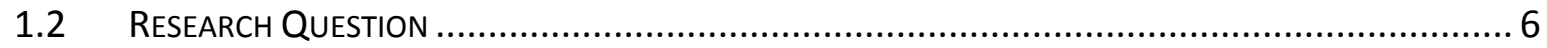

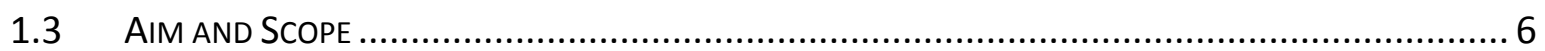

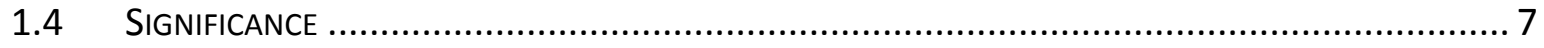

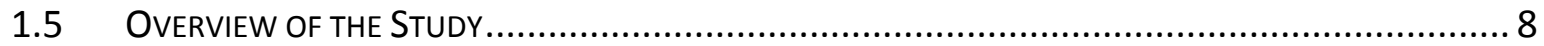

CHAPTER 2: MASS HOUSING FROM ANTICIPATION TO PARTICIPATION ........................... 10

2.1 CHANGING RELATIONSHIPS BETWEEN ARCHITECTS, HOMEBUYERS AND HOUSING .......................... 11

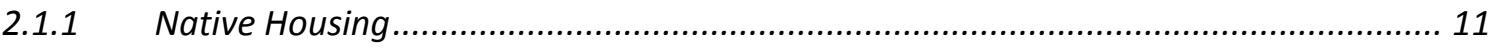

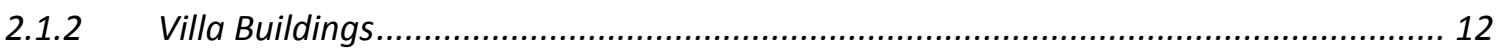

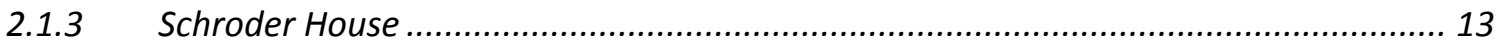

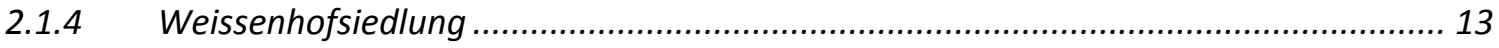

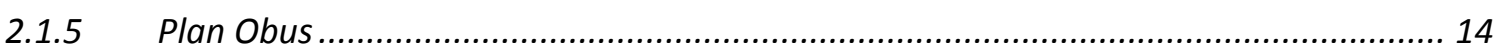

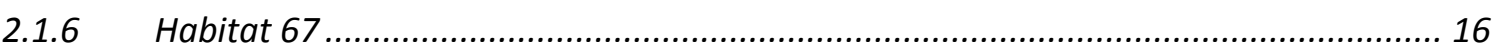

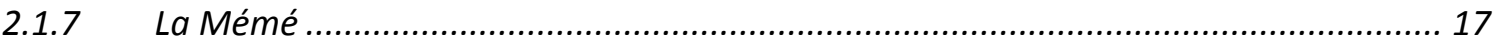

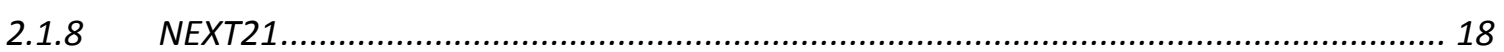

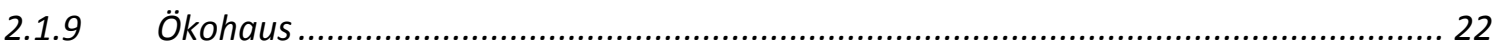

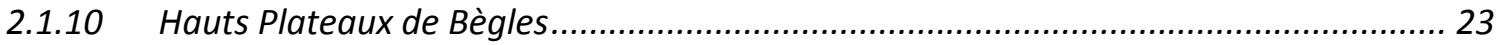




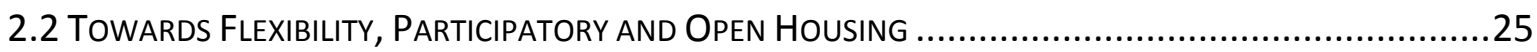

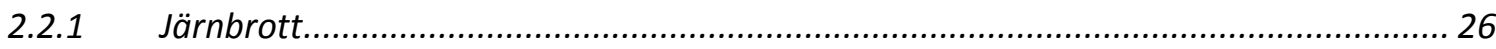

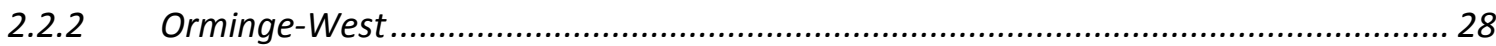

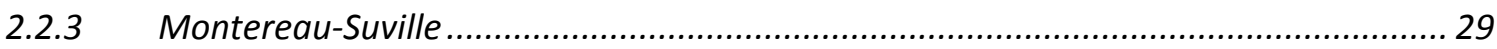

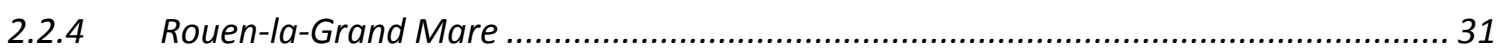

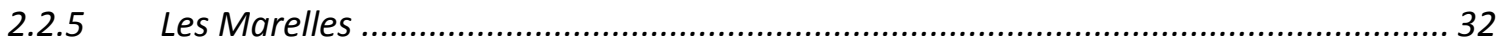

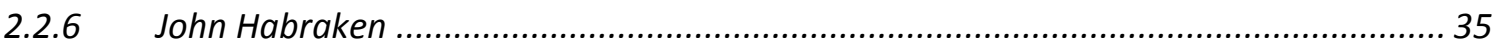

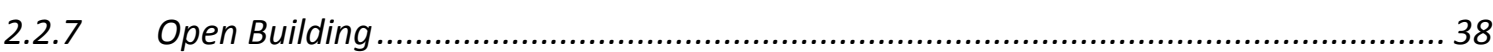

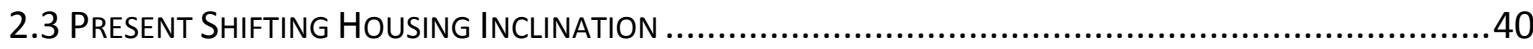

CHAPTER 3: TOWARDS A TECHNOLOGICAL MASS HOUSING SYNERGY ........................... 48

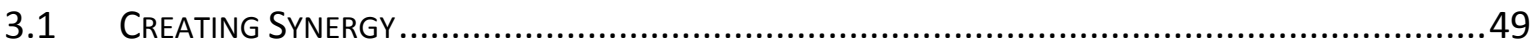

3.1.1 Bringing about Design Participation in other Practices............................................ 50

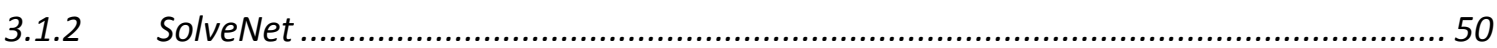

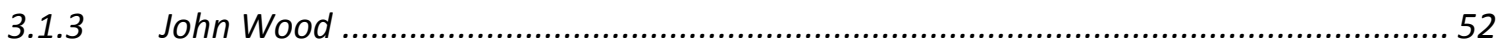

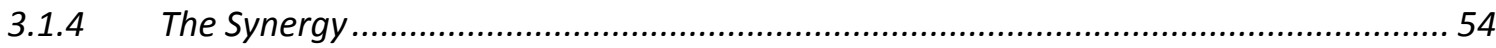

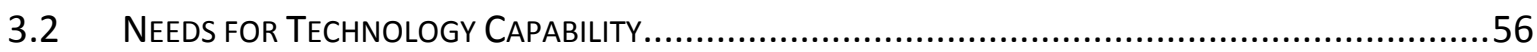

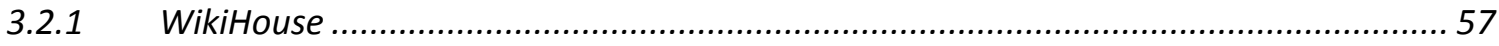

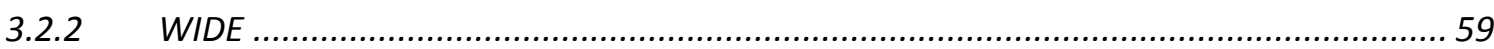

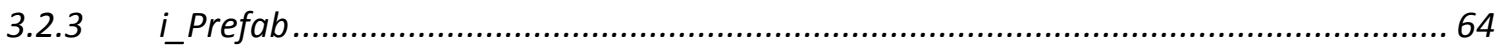

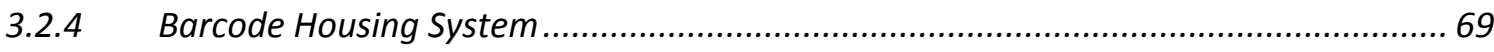

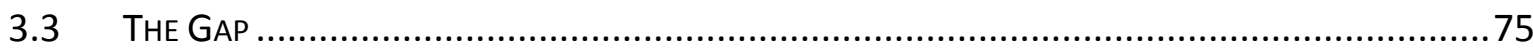

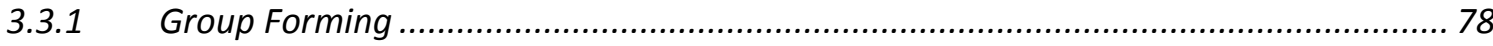

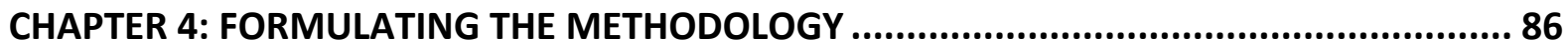

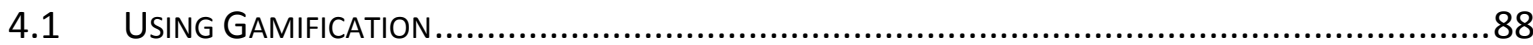

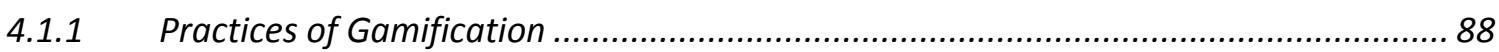

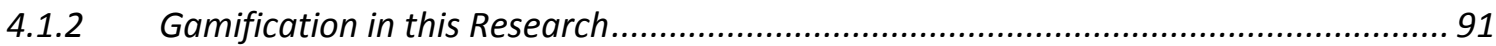

4.1.3 The Eight Core Drives Of Gamification ................................................................... 92

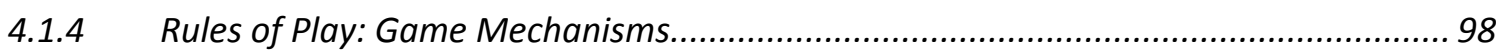

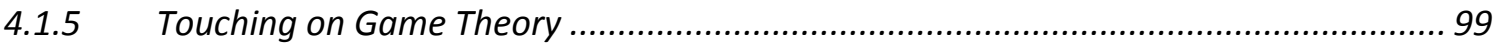

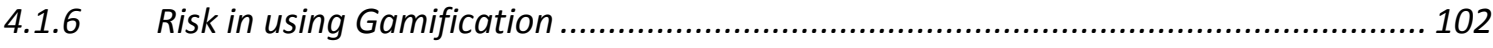

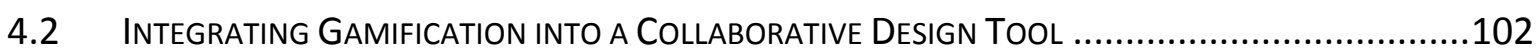

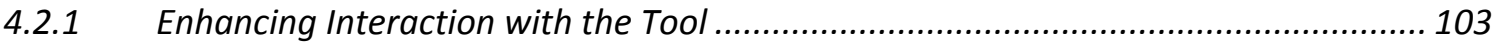

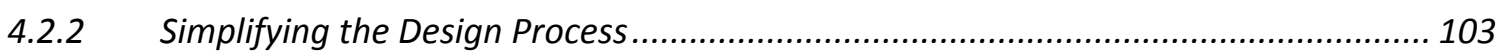

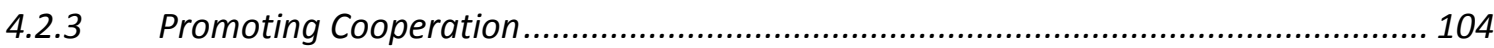

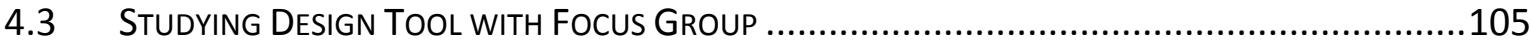




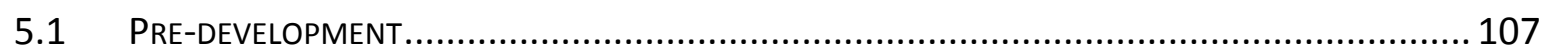

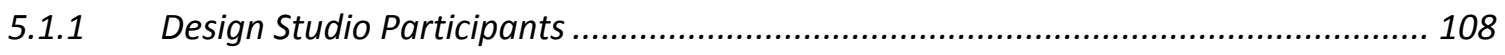

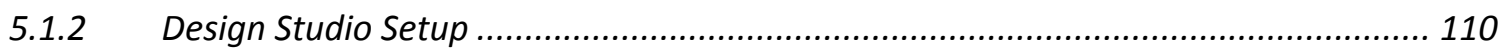

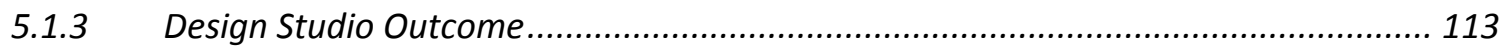

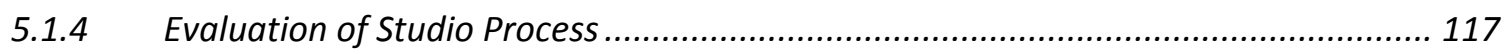

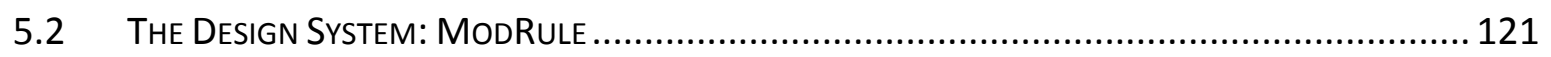

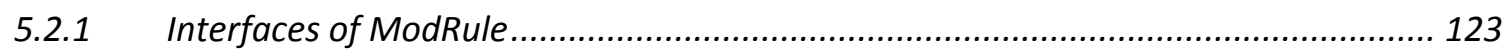

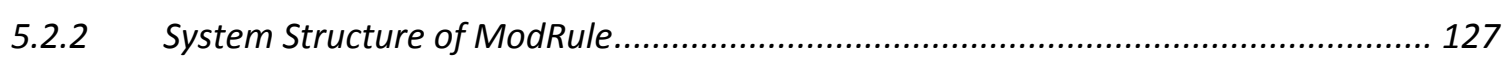

5.2.3 Testing Out ModRule - The Participants................................................................ 128

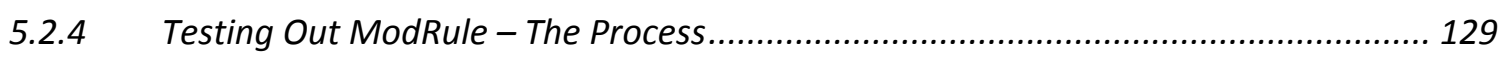

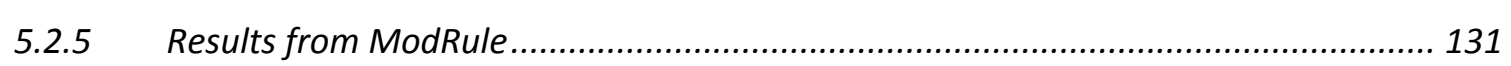

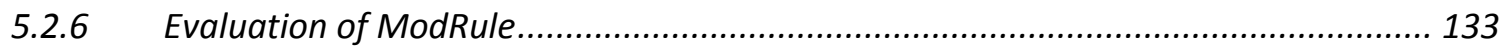

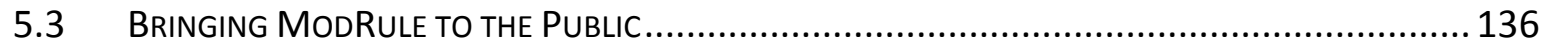

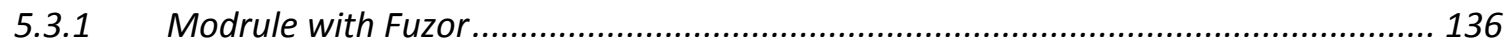

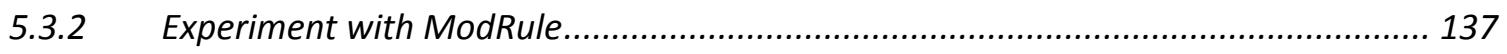

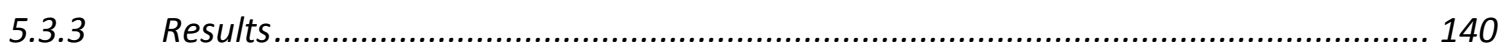

CHAPTER 6: ESTABLISHING A MASS HOUSING METADESIGN .................................. 142

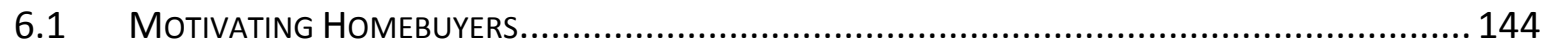

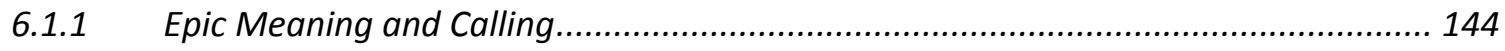

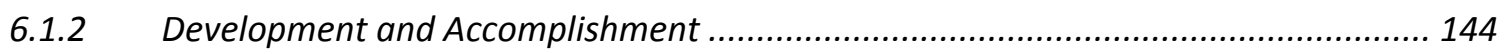

6.1.3 Creative Empowerment and Feedback .................................................................. 145

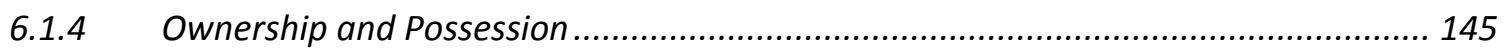

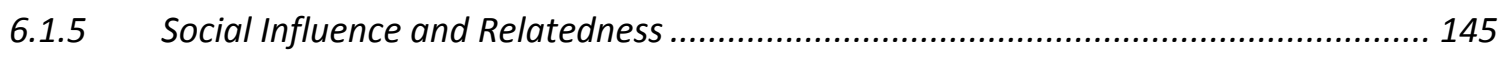

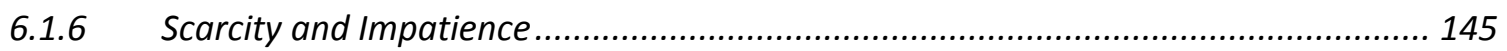

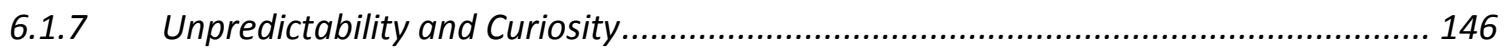

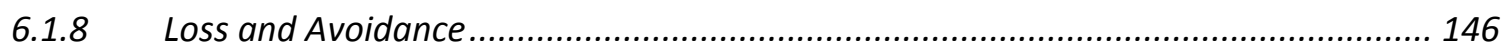

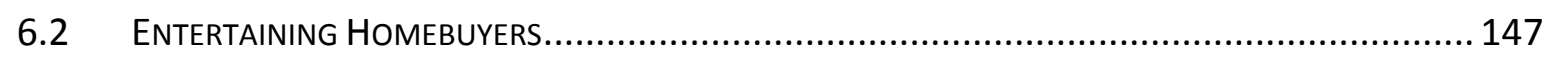

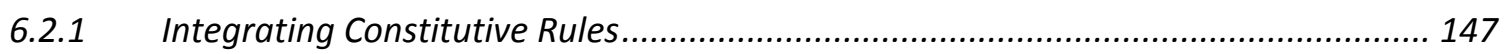

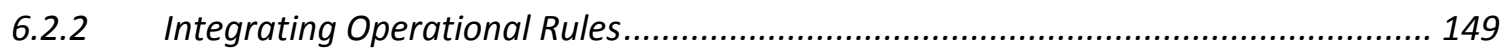

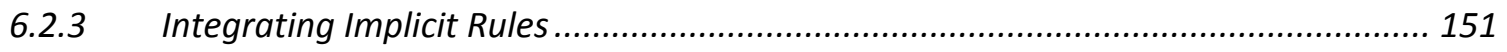

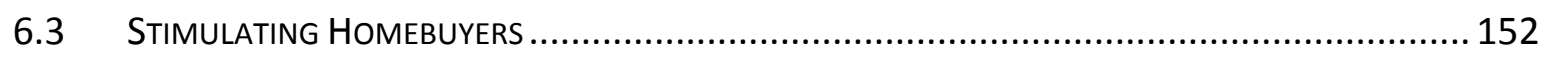

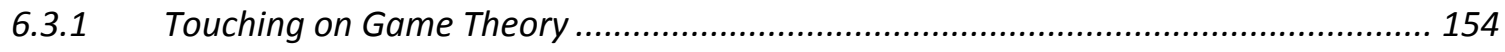

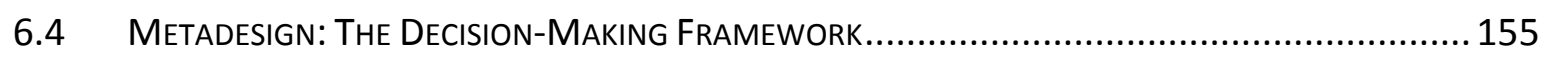

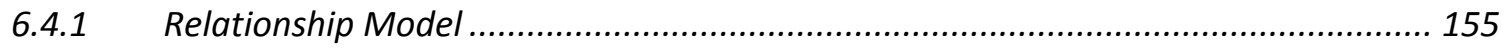

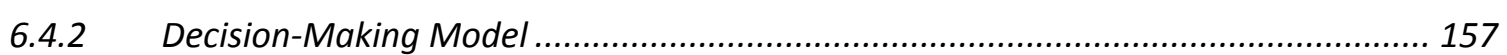

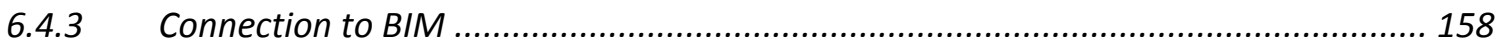




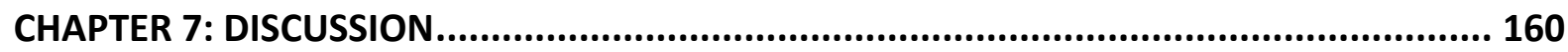

7.1 CONTRIBUtING TO THE HOUSING IMPLEMENTATION DESIGN ENVIRONMENT ...........................160

7.1.1 Bringing Changes to the Built Envrionment ............................................................ 161

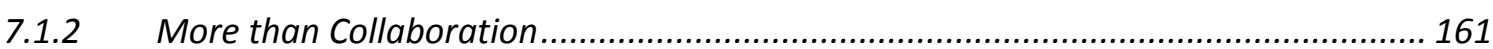

7.1.3 Challenges for Standard Architectural Practices ....................................................... 162

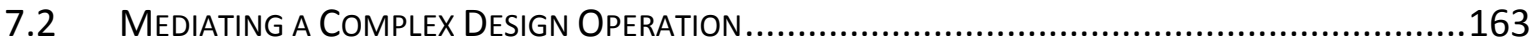

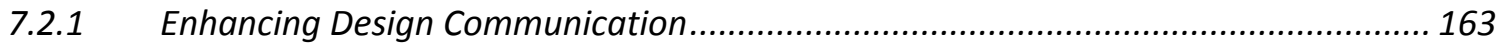

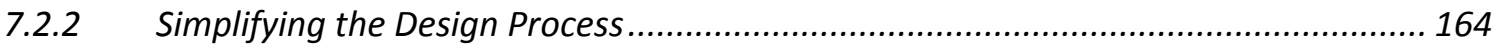

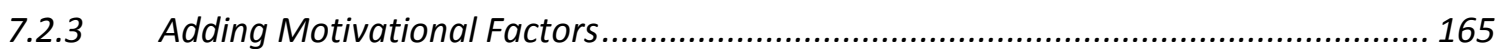

7.2.4 Formulating Design Decision Support Network..................................................... 166

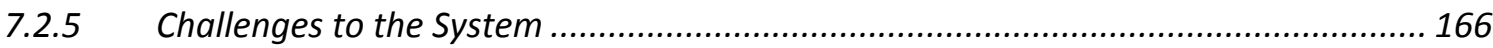

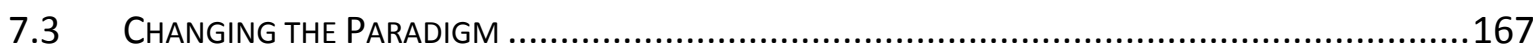

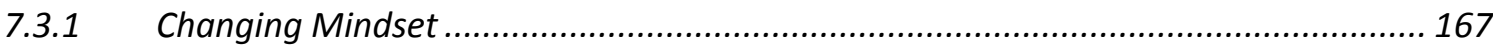

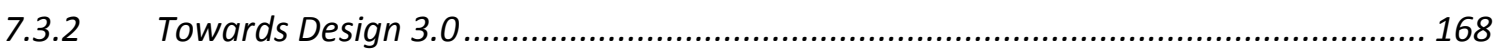

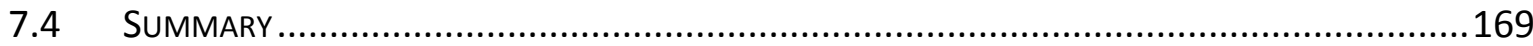

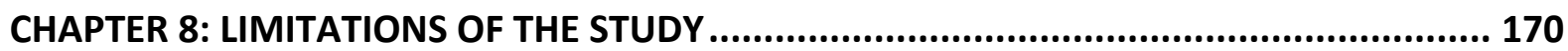

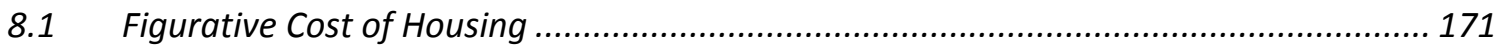

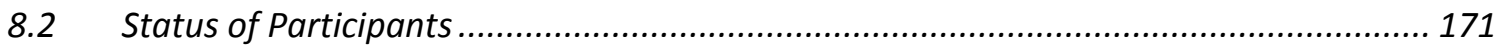

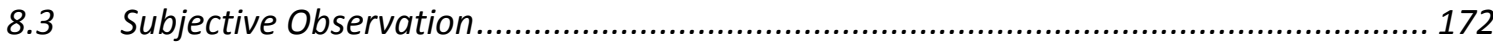

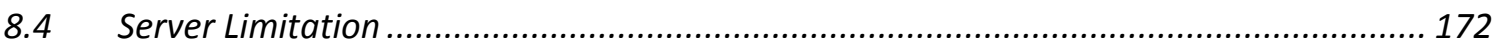

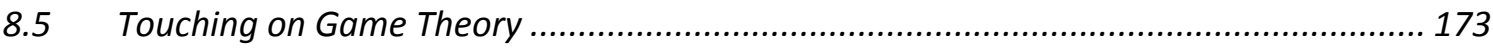

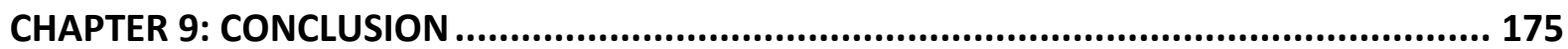

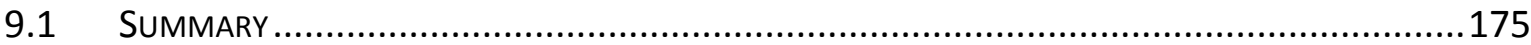

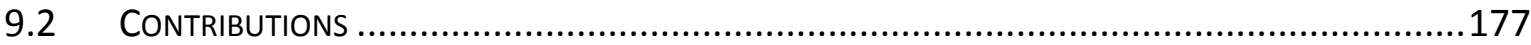

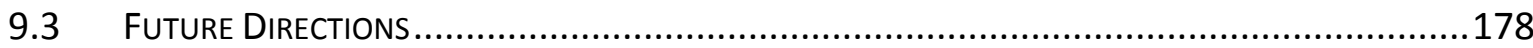

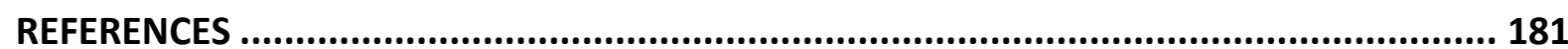

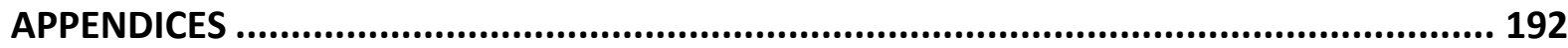




\section{Figures}

Figure 1 Screenshot of 'De Drager / A film about Architect John Habraken' showing architect communicating with a homebuyer

Figure 2 Villa-Building - every unit has its separate garden (Corbusier, 1923)

Figure 3 Flexible apartment of Mies Van der Rohe, Stuttgart, Weissenhofsiedlung (Top) Conventional Plans (Middle) Free plan and continuous space (Bottom) Plain exterior facade (Arch+, Oct 1989)

Figure 4 Plan Obus by Le Corbusier (Image taken on the web) ..... 15

Figure 5 Habitat 67 by Moshe Safdie (Merin, 2013) (Left) Prefabrication construction (Middle) Section of Habitat 67 (Right) Bird-eye view image of Habitat 67.

Figure 6 La Mémé by Lucien Kroll and the students (image by architect) (Left) Elevation of La Mémé showing different façade put together (Middle) Interior of La Mémé showing different size doors (Right) Design process by students ....

Figure 7 Application of flexible spaces with the five CHS layers and the eight-point synthesis (Center for better living, 1982).

Figure 8 (Top-left) Front image of Next21 (Top-middle) Changes of façade (Top-right) Separation of Support and Infill (Bottom) Distinctive differences in floor plans (images by Osaka Gas) . 21

Figure 9 (Top-left) Structure drawing of Ökohaus (Top-middle) Collaboration between homebuyers and architects using physical model of the structure (Top-right) Two images of the final constructed Ökohaus showing its integration with nature (Bottom) Digital model of the final outcome (sol•id•ar Architekten, 1999).

Figure 10 (Left) Concept and vision of Hauts Plateaux (Middle) Comparision of outcome with image drawn by SITE (Right) Final constructed Hauts Plateaux (images by architect) .................. 24

Figure 11 Building of the Järnbrott district, and three plans made by tenants (Krantz, 1976)........... 27

Figure 12 Diverse plans of Stockholm: Orminge-West, 1967-1971 (Krantz, 1976)............................ 28

Figure 13 (Top) Drawing and image of Montereau-Suville (Bottom-left) Plan of Montereau-Suville separated into four plots (Bottom-right) Unit plans by architects(top) and homebuyers(bottom) (Periáñez, 1972) ........................................................................ 30

Figure 14 Rouen La Grand Mare and the GEAI process (Fichelet \& Fichelet, 1973) ........................... 32

Figure 15 Les Marelles in Yerres (1971-1975) (Left-top) Overview and roof detail of Les Marelles (Leftbottom) Image and drawing detail of utilities embedded in structure (Right) Floor plan designed by homebuyers (Maurios \& Herrou, 1976) ........................................................... 34

Figure 16 Diagram of environmental levels in Open Building (Kendall S. , 2004) ............................... 38

Figure 17 Images of Torre David (Brillembourg \& Klumpner, 2012)(Google) ..................................... 42

Figure 18 Winning design for housing competition by Hsieh Ying-Chun (ATD, 2012) ........................ 43

Figure 19 Nodal diagram showing the number of players and the implications to comprehend the

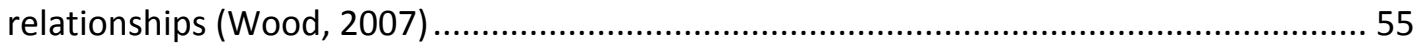

Figure 20 The players and relationships of BedZED (Wood, 2007) ................................................... 56

Figure 21 Assembly of WikiHouse and varied outcomes (Labarre, 2011) (Parvin, 2013) .................... 58

Figure 22 Customization process in Taiwan housing (Chien \& Shih, 2000) .......................................6 60 
Figure 23 System Architecture of WIDE (Chien \& Shih, 2000)

Figure 24 Interface screenshot of WIDE-Kindom (Chien \& Shih, 2000)

Figure 25 Interface screenshot of WIDE-Roadhouse and components of the building system (Chien \& Shih, 2001)...... 63

Figure 26 Existing models of home design and model of i_Prefab (Huang C.-h. , 2007) .. 65

Figure 27 Organisation of $i_{\text {_Prefab }}$ with the levels the questionnaires and its respective interfaces (Huang C.-h. , 2007)

Figure 28 Collaboration of various stakeholders working concurrently within the system, allowing inputs from different users at any stage of the process (Madrazo, Rivera, Costa, \& Sicilia, 2010).

Figure 29 BCHS generating floor plans by merging parametric 'bars' of various functions and the use of open building concepts (ARC Engineering and Architecture La Salle, 2014)...

Figure 30 Screenshot of the four interfaces in Housing Unit Configuration of BCHS (ARC Engineering and Architecture La Salle, 2014)....

Figure 31 Current communication approach between architects and homebuyers with the system established

Figure 32 Proposed approach to achieve constant dialogue and exchange among the homebuyers and the architects within the system

Figure 33 Cell definitions of Group Form (Ong, Janssen, \& Lo, 2013)

Figure 34 Snapshot of the design process of a homebuyer with a video available online on Vimeo (Ong E. H., 2011) (Ong, Janssen, \& Lo, 2013)

Figure 35 (left) current situation and worse outcome possible, (centre) experiment outcome, (right) expected outcome (Ong, Janssen, \& Lo, 2013).

Figure 36 Spatial collaboration outcome generated into a practical plan (Ong, Janssen, \& Lo, 2013) 83

Figure 37 The Eight Core Drives of Gamification (Chou, 2015)

Figure 38 Profiles of some social games and application based on the eight core drives (Chou, 2015)

Figure 39 The three primary schemas (Salen \& Zimmerman, 2003)................................................ 98

Figure 40 Precedent studies by some of the students ................................................................... 110

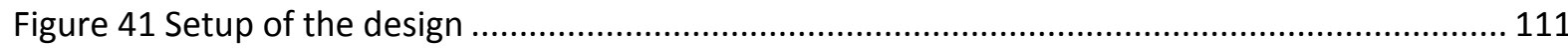

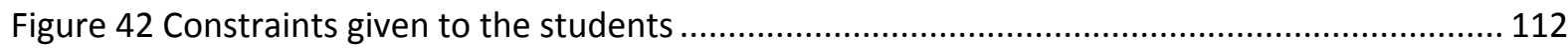

Figure 43 Housing types comprise of various number of units.................................................... 113

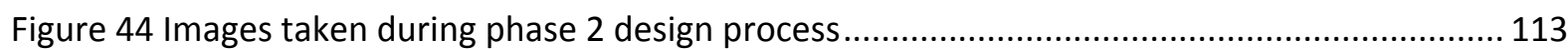

Figure 45 Various stages of design collaboration discussion, top image done digitally by researcher to keep track of the design progress in one of the focus group showing the circulation path decided by the students.

Figure 46 Further design collaboration discussion to work out the design details, progressing towards the building form and finally putting everything into digital to generate the final outcome

Figure 47 Detailed design of every units housing units by one of the students in each focus group 118 
Figure 48 Five major components of mass housing design: spatial driver, structure frame, skin modules, inner partition system and utility system.....

Figure 49 "Create" Interface of ModRule system showing the fixed building core and parameters (coloured) set by the architect....

Figure 50 "Collaborate" Interface of ModRule system showing the what the homebuyers see while interacting with the interface and collaborating with other homebuyers...... 124

Figure 51 "Watch" Interface of ModRule system showing the choices of the various homebuyers and the conflicts in red.

Figure 52 Defining the set of skin module and inner partitions for the homebuyers to select and place them into the space they created....

Figure 53 "Interior" Interface of ModRule system showing the spatial connection of the various functions that one homebuyer hope to have.

Figure 54 The design collaboration workflow with ModRule

Figure 55 The system structure of ModRule

Figure 56 The design form of each of the nine students

Figure 57 Rules and parameters set by one of the designers

Figure 58 Rules and parameters set by another designers.

Figure 59 Final outcome of the chosen design

Figure 60 Linking with Fuzor using its API (Kalloc, 2014)

Figure 61 The system structure of ModRule with Fuzor showing only an additional browser

Figure 62 Setup of the beta testing of ModRule-Fuzor system

Figure 63 Screenshot of the big screen showing the participants the options they chose in ModRule (top) with respect to the site environment shown in Fuzor (bottom)

Figure 64 Screenshot showing one participants placing a wall in a VR environment (top) with a selected site (bottom left) and a prepared outcome to show its possibility (bottom right)

Figure 65 Schematic map indicating the character of Metadesign by Jordan Dalladay-Simpson (Wood, 2016)

Figure 66 The level of implementation of the various core drives in ModRule, the further distance the points are from the centre means higher level of implementation to enhance engagement with homebuyers

Figure 67 The overall mapping of the possible decisions in ModRule. A highlighted path showing one possible set of decisions made by a homebuyer leading to a design option (in this case Type

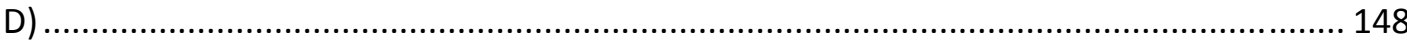

Figure $68 \mathrm{~A}$ tentative operation process of the homebuyers in designing their living space. 150

Figure 69 A screenshot of the game "Gauntlet Legends" showing the attributes of the character class (image from Pinterest) .

Figure 70 Homebuyers profiles created and played by participants from the study of ModRule..... 153

Figure 71 Suggested addition to the parameters to encourage more collaboration 154

Figure 72 Tetrahedron depicting minimum grammar of relations (left) and achieving best outcome (right) (Wood, 2007) 156 
Figure 73 Friedman- adapted (2011) diagram showing the digital intervention in the housing design process as decision support.

Figure 74 The possible connection of the housing design system ModRule to be expanded and connect to BIM with the professionals with architects playing the key role. 159

Figure 75 Model built by one of the groups among the participating students 166

Figure 76 Participants engaging the digital tools, ModRule, within a location 173

Figure 77 Row Housing West 8 in Borneo Sporenburg, Amsterdam (West 8, n.d.) showing a rich design outcome that gives every housing unit a unique outlook.....

Figure 78 Collective façade design by a group of students from a building science design course. An unintentional outcome but a very desirable design solution that this research is hoping to achieve.

\section{Tables}

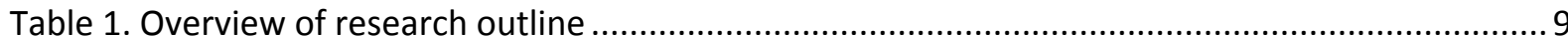

Table 2. Summary of examples used in this chapter with its analysis .................................................47

Table 3. Summary of system described in the previous section .......................................................... 76

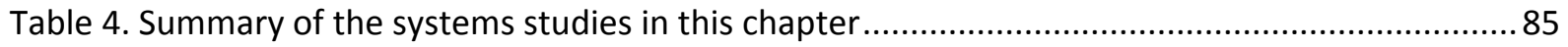

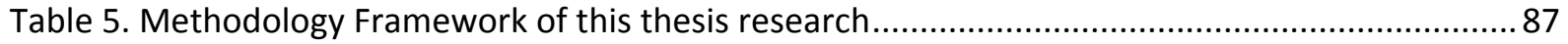

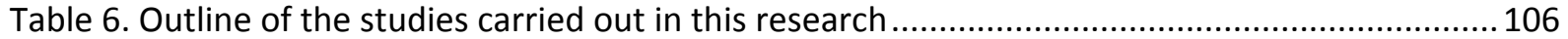




\section{Acronyms}

Acronyms used in this thesis:

2D

Two-dimensional

$3 \mathrm{D}$

Three-dimensional

AR

Augmented Reality

BIM

Building Information Modelling

CAD

Computer Aided Design

CAAD

Computer Aided Architectural Design

$\mathrm{HCl}$

Human-Computer Interaction

SAR Stichting Architecten Reseach

SQL Structured Query Language

VE Virtual Environment

VR Virtual Reality

WebGL Web Graphics Library 


\section{Glossary of Terms}

Definition of terms to clarify its meaning in this thesis:

Mass housing

High-rise

High-density

Homebuyer

Bottom-up

Top-down

Architect(s)
Housing for the mass, NOT specifically for the low-income

Housing/building that are multi-storey and equipped with elevator

Building with a great number of apartments units stacked on one another

Individual or family who is buying or renting an apartment units for inhabitation of at least a few years

Design approach that is clients-oriented where their needs will be the starting point of the design

Design approach driven by a person or an organisation. The design will be almost totally influenced by that person's style and taste. On an organisation level such as a government, the design could be driven based on directives.

A professional who engages in the field of architecture. In this research, this professional is not an individual but include the design team behind within a design firm. But does not include anyone from other professions. 


\title{
Prelude: Research Background \& Personal Motivation
}

\author{
How can man, as a poor terrestrial, manage to house himself? \\ Can one not achieve this same quality within the urban area at a high density? \\ - Frei Otto - \\ (2012)
}

This $\mathrm{PhD}$ thesis has been a persistent journey to fulfil a personal dream. It is a very simple dream, one which many others might share: to own a house. What it means 'to own a house' has changed as economies flourish and cities become ever denser. At present, 'to own a house' requires an average middle-income person a few decades to save enough or pay off the mortgage. Even when acquired, this 'house' is often an apartment unit within a high-rise building.

I am at the stage of my life where I am newly married and looking for a place to settle down in after graduation. This can be time-consuming and frustrating. Even though we have cleared the financial problem, this is just one part of a big picture. Our key obstacle is finding a home that suits our needs. We searched countless apartment units, but most fail to meet our requirements. The luxurious apartments provide the necessities, but also possess unnecessary functions that we cannot remove. As this place will be our home for the next few decades, it is natural for us to want something that suits our needs. Friends and relatives going through the same search process, all ended up with the same outcome: give up searching; take whatever is available; adapt to the space.

As a practising architect, it is a great challenge to provide a design to suit the living needs of every family. A typical floor plan with minor adjustments is usually applied to fit the majority. As a house-seeker, I do not have the means to design my own living space if I choose to live in an apartment. After years of architecture education and practice designing for people, there is still an apparent gap between these two main areas; architects trying to understand the individual living needs of individual people, and individuals wanting the capability to create their own living space. Whenever housing is put on the table for discussion, the focus is usually on meeting housing demands for the population, or dealing with the cost of housing stock. It 
seems that the common belief is that as long as you have a place to live in, you should be happy. This should hardly be the case, especially when housing is one of the basic needs of living, and a need that could take a person's lifetime to achieve.

This then, is the main motivation for my PhD research. There are numerous studies that try to cater for individual living needs, although most of these work only for individual houses and are not applicable to high-rise apartments. The complexity of high-rise housing makes it a totally different ballgame, from design to construction, and since the stakeholders for such a project are manifold, there are just too many limitations for any studies to proceed far. My main concern is the connection between the occupants and the design process, a connection through which individual needs can be answered.

A key stakeholder, one with the most varied unknown factors are the occupants. I imagined myself designing for the families of my group of friends. It is a major challenge to include all the different needs and requests into one building design. The time and effort for gathering information is too consuming.

My main interest in computational architecture brings me to considering a digital platform as a means of gathering information. It would be most efficient if the occupants themselves initiate the process willingly, just as I would do as a house-seeker. This bring us to the next challenge; how to motivate fellow house-seekers to collaborate without any professional design knowledge.

Here is where gamification becomes the key strategy for my research.

This thesis will explain in detail the research process examining how gamification and a digital medium can bridge the gap between occupants and the design process. My hope is for the proposed method to be implemented in the future for house-seekers like me to realise their dream of living space in the city. 


\section{Published Works}

Publications deriving directly from this research

\section{JOURNALS}

Lo, T. T., Schnabel, M. A., \& Moleta, T. (2018). Digitally-Built Housing Communities. How Possible Is It? Housing the Future book, by Graham Cairns, 10pgs. Libri Publishing (accepted, awaiting publication)

\section{BOOK CHAPTERS}

Lo, T. T., Schnabel, M. A., \& Gao, Y. (2015). ModRule: A User-Centric Mass Housing Design Platform. In G. Celani, D. M. Sperling \& J. M. S. Franco (Eds.), Computer-Aided Architectural Design Futures. The Next City - New Technologies and the Future of the Built Environment: 16th International Conference, CAAD Futures 2015, São Paulo, Brazil, Selected Papers (pp. 236254). Berlin, Heidelberg: Springer Berlin Heidelberg.

\section{CONFERENCE PAPERS}

Lo, T. T., Schnabel, M. A., \& Gao, Y. (2013). Collaborative Mass Housing Design Practice with Smart Models. International Conference on Digital Architecture (DADA), China.

Lo, T. T., \& Schnabel, M. A. (2013). Definition of Smart Parametric Model for Collaborative Design of Mass Housing. Cutting Edge: 47th International Conference of the Architectural Science Association (ANZAScA), (pp. 207-216). Hong Kong.

Lo, T. T., Schnabel, M. A., Aydin, S. \& Shi, K. (2014). MODRULE: Using Gamification for Collaborative Mass housing Design Process. Across: Architectural Research through to Practice: 48th International Conference of the Architectural Science Association (ANZAScA), (pp. 733-743). Italy.

Lo, T. T., Schnabel, M. A., \& Gao, Y. (2015). Digital Factory for the People: The Application of BIM. International Conference on Digital Architecture (DADA), Shanghai.

Lo, T. T., \& Gao, Y. (2015). ModRule: Sustainable Mass Housing System for the People. 11th China Urban Housing Conference (CUHC), Qingdao

Lo, T. T., Schnabel, M. A., \& Aydin, S. (2016). Modrule: A Gamified Design Communication Platform. Design Communication European Conference (DCAe), (pp. 339-347). Istanbul.

Lo, T. T., Schnabel, M. A., \& Moleta, T. (2016). Establish, Exchange and Engage: A Support System for Multiple Decisions to Co-produce. 13th International Conference on Design \& Decision Support Systems in Architecture and Urban Planning, Eindhoven.

Lo, T. T., Schnabel, M. A., \& Moleta, T. (2016). A Simple System for Complex Mass Housing Design Collaborations - A system development framework. Complexity \& Simplicity - Proceedings of the 34th eCAADe Conference - Volume 2, (pp. 137-146). Oulu. 
Lo, T. T. (2016). Building Communities through Inclusive Design Platform. Future Housing - Global Cities and Regional Problems - Architecture, Media, Politics, Society (AMPS) Proceeding Series 7, (pp. 196-205). Melbourne.

Lo, T. T., Schnabel, M. A., \& Moleta, T. (2017). Gamification for User-Oriented Housing Design - A Theoretical Review. Protocols, Flows, and Glitches - Proceedings of the 22nd CAADRIA Conference, (pp. 63-72). Suzhou.

\section{SOFTWARES}

Lo, T. T., \& Shi, K. (2014 - 2017). ModRule [Web-based system]. http://modrule.com

\section{WEBSITES}

Lo, T. T. (2014, Aug 4). Parametric Design Thinking (Mass Housing Context). Retrieved from Facebook: https://www.facebook.com/groups/digitaldesignthinking/

Lo, T. T. (2017, Jul 8). Social Gaminology. Retrieved from Facebook:

https://www.facebook.com/groups/463848703970289/

\section{APPLICATIONS ADAPTED IN THE CONTEXT OF THIS THESIS}

Yu, H. (2013 - 2018). Fuzor [Computer Software]. https://www.kalloctech.com/index.jsp

\section{OTHER MEDIA}

Lo, T. T., Schnabel, M. A., \& Aydin, S. (2014). Collaborative Design with Quasi-Grammars. Rethinking Comprehensive Design: Speculative Counterculture, Proceedings of the 19th International Conference on Computer-Aided Architectural Design Research in Asia (CAADRIA 2014), (pp. 941-942). Kyoto.

Lo, T. T., Schnabel, M. A., \& Moleta, T. (2016). Multi User Online Design Environment for Mass Housing - A collaborative decision-support tool. Complexity \& Simplicity - Proceedings of the 34th eCAADe Conference - Volume 1, (pp. 77-80). Oulu. 


\section{Acknowledgement}

I would like to express my sincere gratitude to my supervisor, Professor Marc Aurel Schnabel, for his confidence in me, his endless, patient support throughout my PhD journey, and for his guidance through many critical moments in and outside of this work. I am also very grateful to my secondary supervisor, Tane Moleta, who has constantly encouraged me and pushed me forward with his kind words and positive attitude.

I am truly blessed to have met my wife, Qiu Yan, during the journey of this PhD. She has brought joy and colour into my life, making my experience so much more memorable. I dedicate most of this $\mathrm{PhD}$ to her, for she has made a great many sacrifices to follow me through this tough journey, and been understanding and supportive.

I must thank my parents and my siblings for their strong support; they are the pillars of my life. I also would like to thank my in-laws, who have known me only for the two years since I met my wife and yet who have already provided so much support in my PhD studies.

In addition, I would like to thank all the friends and colleagues I have met and who have made my days through the PhD journey memorable: Serdar Aydin for being a caring friend; Zhang Yingyi, Shuva Chowdhury and Harry He Yi for sharing the joy and pain; Chen Yong Ming for constant drive and passion for research.

I also thank mentors who have helped me in various phases of my study: Professor Gao Yan for exposing me to the practical aspect of design research; Professor Tsou Jin Yeu for giving me deep insight into the field of high-rise housing; Professors from CAADRIA conferences such as Andrew Li and Nik Chien who have provided me with great advice.

Finally, I would like to acknowledge Kalloc Studio, the development team behind Fuzor, for providing all the technical support during my $\mathrm{PhD}$. I would especially like to thank Henry $\mathrm{Yu}$, the CEO of Kalloc Studio, for his constant support of my research and development. 



\title{
Chapter 1: Introduction
}

\begin{abstract}
Families living in private houses often take photographs of their children at the front door for example on their first day at school. However, they do not feel like taking photos in front of their homes in communal housing blocks. I am sure that the families living in NEXT21 will take photos on such occasions. I think this shows that this form of housing can have an identity and be loved by its residents and that will be remembered for a long time.
\end{abstract}

\author{
-Seiichi Fukao- \\ Professor of Tokyo Metropolitan University \\ NEXT21 team (2015)
}

At present, 'a house' may be simply an apartment unit within a high-rise building. To meet high demand, housing has been simplified to prefabricated, mass-produced 'containers' for shelter and living. However, values and needs differ widely across cultures. Differences also exist in spatial needs (Nasar \& Kang, 1999) (Vulture, 2014).

Family structures in contemporary societies come in various forms; the typical modern 'two parents, two children' families are becoming less common. With globalisation, these differences can be seen even more clearly. One such difference is interracial marriage; there is much research considering this diversity in America, but racial demographics are changing around the world (Ratliff, 2017). Another change is globally decreasing marriage and birthrates (World Family Map, 2014). This could mean an increasing divergence of housing needs.

The industry is currently in such a top-down operating state that occupants usually cannot have any input into the design process; they can choose only from predefined options; selecting the one most suitable for them. Although every family is different with diverse needs, houses are usually categorised quite generally into studio apartments, and 3-room to 5-room apartments. Instead of design responding to family needs, the opposite situation is the norm: the family must adapt itself to the units' design. This results in many mass housing designs reacting ineffectively to multi-faceted social needs, 'forcing' people to live in identical units designed and prefabricated for efficiency and affordability (Gao, et al., 2015). 
Prefabrication is a construction technique that became popular during the post-World War II period to quickly meet the high demand for new accommodation after houses were destroyed during the war (Hayes, 2014). This technique has since evolved and is now applied to building high-rise housing for cities with fast-growing populations increasing their density. For example, the Singapore government established the Housing Development Board (HDB) to provide fast and affordable housing through the prefabrication method as a form of nation building (HDB, 2016). Similarly, and more intensively in Hong Kong, prefabrication is a key strategy for meeting the high demand for affordable housing. This technique fulfilled its primary purpose to supply for the high housing demand (HKHA, 2016). However, it is not a long-term solution. In the UK, for example, prefabrication is still regarded as a 'make-do alternative to serious construction' (Hayes, 2014). Housing objectives have transformed from just providing a roof over people's head to including conditions and performance standards that will eventually give everybody high quality accommodation. Architects have put in the effort to provide limitless design possibilities to meet an individual's needs, wants and budget; masscustomization was developed to provide such capabilities. However, this is yet to apply to highrise. Prefabrication is about uniformity, and it is no longer flexible. In the Netherlands, where there is a strong focus on the housing industry, uniform floorplans have slowed the development of a truly industrialised system (Habraken, 2002). A system that can be achieved through the distinct separation of infill systems from the open building.

The result is the idea of Open Building (OB). This approach, developed in the sixties for building design, is internationally recognized and has come to represent a new wave in the architectural field (Kendall S. , 2004). It was developed by John Habraken (1961) who created an architectural research foundation, SAR. The term $O B$ is used to indicate a number of different but related ideas that surround this kind of environment-making. These include: (Habraken, 2000):

- The idea of using distinct levels of intervention in the built environment such as 'support' and 'infill'. 
- The idea of including users / inhabitants in the design decision process.

- The idea of design collaboration among different kinds of professionals.

- The idea of flexibility in the technical system, allowing interchangeability between one system with another performing the same or different functions.

- The idea that the built environment is constantly transforming, and that it is necessary to embrace changes.

- The idea that the design process for the built environment is endless and transforming part by part.

The main objective of his research is to promote a flexible design that could see all players collaborate in the design-build-promoting process. OB provides the necessary foundation to ground this study.

This research proposes that the main reason for housing failing to provide for the needs of the people, is the exclusion of the people who will live in the building from the design process. Participation is, therefore, the central topic of this research. In the past, architects spent time with house-owners, discussing their needs and preferences before a design was built (Figure 1). Now, high-rise occupants are not just one family but hundreds living together in one building. Occupants' needs are surveyed and studied, but occupants are not involved in design. The experts make all the decisions and try to design the best 'one-fits-all' apartment based on the study results. John Habraken suggested that such a housing model is outdated and a new model required for the future (Luthi \& Schwarz, 2013); one which includes the participation of the occupants. 


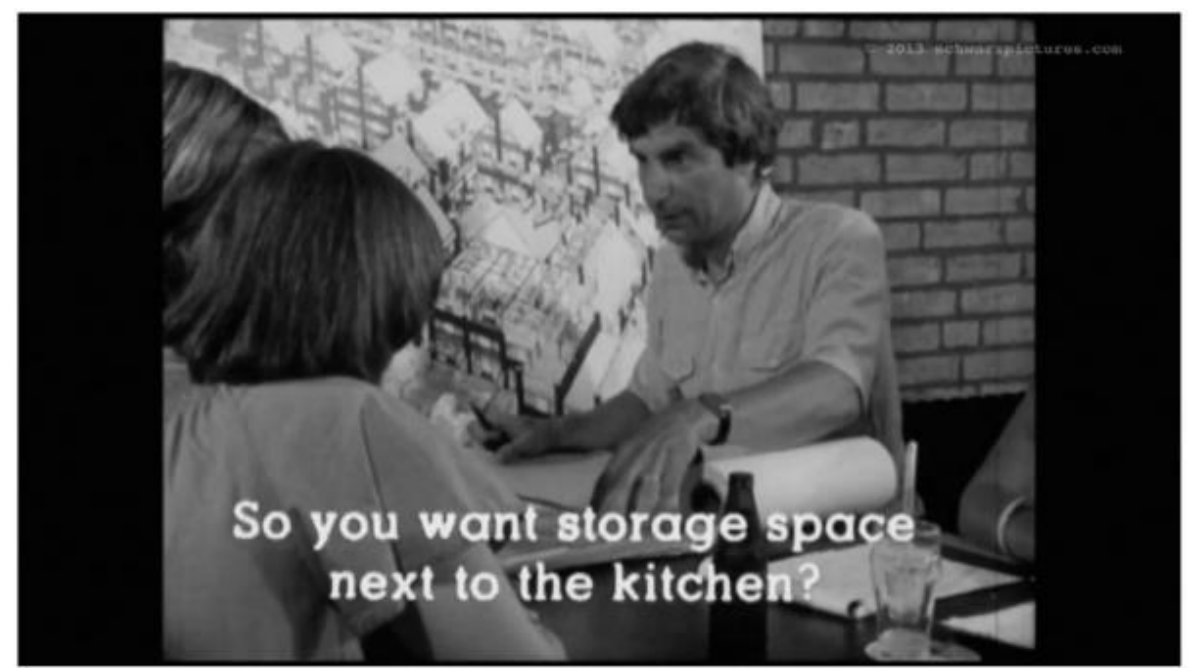

Figure 1 Screenshot of 'De Drager / A film about Architect John Habraken' showing architect communicating with a homebuyer

Participation of homeowners in the multi-family residential building is not new. One very successful example is Frei Otto's Ökohaus project (Eco-home) in Berlin, Germany. It is collective housing which exercises user participation and open design. Frei Otto sees this as an opportunity to consider new ways of living in a high-density urban context. Every occupant participates in the design with the help of the architects. Just like they are building a detached house. Next21 is another experimental multi-family housing project, in Osaka, Japan. It demonstrates the new concept of multi-family housing units that incorporate sustainable design methods and advanced technologies, allowing occupants to participate in the design process and have their needs met as much as possible. In Belgium, Lucien Kroll, a Belgium architect, also demonstrates the importance of a participatory process through his La Mémé student accommodation. Although these examples are not high-rise apartments, they are all multi-users housing typology. The charm of participatory approaches is clear, yet its practice is seldom undertaken. The problem lies in the time and effort required for such a process. These three examples took at least a year just to engage the homebuyers. This research identifies that the development of a participatory process has great potential to be adopted into a high-rise scenario. The main obstacle is the management and coordination of a wide variety of occupants. This research, therefore, identifies the need to increase the efficiency of design 
participation in creating flexible yet cost effective mass housing, and that a digital platform could be a solution.

Technology advancement has pushed architecture design to the digital era. Parametric modelling tools have allowed building designs to come in any form and shape, as long as the architects can justify it. Building Information Modelling (BIM) has allowed close collaboration between building stakeholders, and vastly improves the efficiency of building construction. Yet the inclusion of homebuyers in the design process is yet to come about. Prefabrication has produced considerable change, but mostly in improving cost savings and accuracy, spurring more housing of this typology. Still, there is a handful of research that tries to develop a digital system to improve the design outcome and engage the homebuyers. Benros (2007) developed a system that could generate potential floorplans based on a specific algorithm, diverging the outcomes to isolate one that suits the users and fits the overall context of the building. "Barcode housing system" (Madrazo et al., 2009) allows the prospective occupants to adjust their plan layout according to their needs. However, the systems developed either fail to engage the homebuyers directly, or contain too many constraints - obstructing the desired variety. Most importantly, the designs were not high-rises. There is a challenge in developing an integrated system for homebuyers. How to develop a system that is comprehensive enough to include most housing design details for an immersive design experience and a practical outcome, with a process that is sufficiently simple for anyone to engage with intuitively?

\subsection{Problem Statement}

To date, there is insufficient research examining the connection between user-participation and actual use of digital tools in the design process. If user-participation does indeed make a difference to the design process with the help of digital tools, it is important to understand the correlation between these factors. In summary, if we could systematically integrate the pieces of information derived from research into the characteristics of digital tools, user-participants' background characteristics and the design process, it would be intriguing to know the degree 
to which these factors account for the development of digital tools and the extent to which userparticipants are actually immersed in the design process.

\subsection{Research Question}

To respond to such a problem, this research focuses on a specific question: How can homebuyers design their living space collaboratively in the development stage of residential building, in the presence of the architects through the use of computational tools within a virtual environment?

There is a huge variety of computational tools within the virtual environment. Every tool is developed to serve a particular purpose. To engage in the entire housing design process, however, demands more than a simple tool. A clear narrative of the process is necessary. To immerse participants in the journey sufficiently to acquire a fitting design requires a certain level of enjoyment. A reasonable amount of challenge could bring about a sense of achievement. Being high-rise, design collaboration is unavoidable, creating the need for enhanced capability for communication. All these characteristics point toward one method: gamification.

This research proposes the integration of gamification strategies into a digital housing design system. The speculation is that the nature of gamification could enhance the participatory process and provide an immersive design experience for the architects and, more importantly, the homebuyers. The research question therefore concentrates on how can homebuyers design their living space collaboratively in the development stage of residential building in the presence of the architects through gamification within a virtual environment?

\subsection{Aim and Scope}

The aim of this research is to critically assess the digital interaction and design operations for apartment living space in order to identify factors that can contribute to the development of an engaging digital tool, suitable for collective design in a high-rise residential context. 
As housing is a substantial topic, it is important to set specific scope to the research. Homebuyers in this research are only those who are buying for their own inhabitation. Even purchasing as a rental, they must occupy the space for at least five years. This is a decent period, referencing Singapore's HDB regulation of purchasing government public housing. Next21 also sets its minimum occupancy period to five years.

Ideally, the desired system outcome allows collaboration among various stakeholders, both professional and public, but this research targets only the communication between homebuyers and the architects who design the overall outcome of the residential building.

As practical as this research hopes to be, there is a limitation to how much construction and building standards the design can include. There is also the issue of different regulations in different countries. In general, the focus is on the design engagement process. Therefore, generic structure and utility designs are applied in the building design.

There is a crucial limitation resulting from the nature of this research. As there is no physical building underway, the participants are only imagining the process of acquiring a home. The real nature of public engagement might not be truly reflected in the result, but the discussion among the participants provides valuable insights to the potential development of such a digital tool.

\subsection{Significance}

One intended outcome of the study, on a theoretical level, is to identify a preliminary set of factors that arise out of extended exposure to a different housing design approach, based on the development of participatory engagement. A second intended outcome of the study is to develop a research methodology that will enable efficient investigation of the ability of homebuyers to engage with a complex housing design process. The third intended outcome further contributes to the development of a digital housing design tool by articulating a set of gamification guidelines based on game design rules. There are already findings from various studies demonstrating the significant impact that gamification has on participants' responses 
to different forms of digital tools. Finally, a further concern of the research is the sequencing of the overall metadesign of this kind of participatory housing design process.

In a wider context, this research could provide insights into the relationship between the bottom-up, participatory design approach and the top-down, architects-oriented design approach. On the one hand, the participatory process provides a great opportunity to bring people together to design their living space. They could communicate with each other and get to know the other homebuyers going to live in the same building before moving in. The significance of this bottom-down approach is that it could bring about community building in the pre-occupancy stage. On the other hand, this design method will challenge the role of the architects, but it is important to note that the role of the 'architect' has been changing over time. Instead of playing the original role of designing the whole building, the architect could play more of a 'coordinator' role, organising the homebuyers sufficiently to design the building collectively and collaboratively. Most significant in this top-down approach, is that by attaching the same weight to all relevant aspects; the architects can play a more central role.

\subsection{Overview of the Study}

This thesis consists of eight further chapters within three main parts. Part 1 (Chapters 2, 3 \& 4), situates the current study in related literature and establishes the research methodology. In Chapter 2, the theoretical underpinnings of housing design and the participation of homebuyers are discussed to guide the enquiry. This includes a critical review of the current practice, the transforming context of housing and the place of participatory practices. It also addresses the issues with integrating participatory practices into housing design processes. Chapter 2 argues for the need to investigate the place of participatory practices in the whole process, from housing design to construction to occupancy, and into post-occupancy building maintenance. Chapter 3 examines the need to adopt technology for the participatory process. A number of developed systems are examined to identify significant gaps in the literature. A cross-disciplinary approach is undertaken to draw on areas such as Human-Computer Interaction $(\mathrm{HCl})$, Building Information Modelling (BIM) and providing choices for decision- 
making. Combining insights from the preceding chapters, Chapter 4 clarifies the deduction of the research question. The method of using gamification as the key strategy is explained in detail. This chapter also deals further with the methodological issues and research design that provide the theoretical and procedural consideration in the collection, presentation and analysis of data.

In the second part (Chapters $5 \& 6$ ), this thesis presents the results and data analysis from the studies conducted at the different stages of tool development. The overall metadesign, system framework, technical components and design rules are detailed as open source for any interested parties to further develop such a tool in the future. The last section (Chapters 7 , $8 \& 9)$ contains the analysis, discussion and conclusions drawn from the current study. In Chapter 7, the discussion of the key findings is expanded and appropriate concepts integrating gamification into a participatory housing design tool are developed. Chapter 8 draws upon the challenges faced during the studies of the tool and the limitations of developing the tool to inform the implications of, and recommendations for, ongoing digital development. Finally, Chapter 9 provides the conclusions and reflective evaluation of the study and suggests further research agendas. Table 1 gives an overview of the research:

\begin{tabular}{|c|c|c|c|c|c|c|c|}
\hline \multicolumn{3}{|c|}{$\begin{array}{c}\text { Part } 1 \\
\text { Establishing the Research }\end{array}$} & \multicolumn{2}{|c|}{$\begin{array}{c}\text { Part } 2 \\
\text { Conducting the Research }\end{array}$} & \multicolumn{3}{|c|}{$\begin{array}{c}\text { Part 3 } \\
\text { Analysing the Research }\end{array}$} \\
\hline Chap 2 & Chap 3 & Chap 4 & Chap 5 & Chap 6 & Chap 7 & Chap 8 & Chap 9 \\
\hline $\begin{array}{c}\text { Literature } \\
\text { review of } \\
\text { Housing }\end{array}$ & $\begin{array}{c}\text { Literature } \\
\text { review of } \\
\text { Digital } \\
\text { Tools }\end{array}$ & $\begin{array}{l}\text { Deriving } \\
\text { Research } \\
\text { Methodology } \\
\text { based on } \\
\text { Research } \\
\text { Gap }\end{array}$ & $\begin{array}{l}\text { Studying } \\
\text { the } \\
\text { participation } \\
\text { process } \\
\text { with the } \\
\text { developed } \\
\text { tool }\end{array}$ & $\begin{array}{l}\text { Establishing } \\
\text { a general } \\
\text { framework } \\
\text { from the } \\
\text { study }\end{array}$ & $\begin{array}{l}\text { Discussion } \\
\text { of findings }\end{array}$ & $\begin{array}{c}\text { Challenges } \\
\text { and } \\
\text { limitations }\end{array}$ & Conclusion \\
\hline
\end{tabular}

Table 1. Overview of research outline 


\section{Chapter 2: Mass Housing from Anticipation to Participation}

According to Vitruvius, the Roman master builder, architecture was the combination of three virtues: utilitas, firmitas and venustas. He forgot humanitas [...]

That, I felt, needed changing.

-Lucien Kroll-

(2016)

This chapter expands on the research background introduced in Chapter 1. Here, a brief history of housing is outlined to show the evolution of housing design with efforts to include homebuyers. The aim is to investigate ongoing changes and identify the key characters promoting participation in housing design. The list of precedents in this chapter informs that architects started to include homebuyers in the mass housing design process through flexible spaces. Homebuyers anticipate by modifying the flexible spaces to adapt to their needs. However, there are limitations to how much homebuyers can do. Later precedents show that architects then further the idea to include the homebuyers by having them participate in the development phase of the projects. Note that the list of the key projects closely references an online article by Professor Manuel Periáñez which is in French (Periáñez, 2013) and provides an extensive timeline of the evolution of habitat. The examples selected are reinterpreted for this chapter and only key points are extracted to focus specifically on links relevant to this research topic.

Structurally, this chapter consists of three thematic sections with relevant sub-sections. The first section outlines a brief history of evolving housing design contributing towards the participation of homebuyers. Some key precedents of built works or ideas display the degree of implementation of various concepts. The second section focuses on the issues faced in participatory housing design processes, and the establishment of organisations and groups that have tried to tackle these problems. A discussion in the third section pinpoints the gaps in existing research and the opportunities for applying alternative or enhanced strategies to the 
problem. To prevent any ambiguity in the understanding of this research, key terms are clearly defined in this chapter too.

\subsection{Changing relationships between architects, homebuyers and housing}

Mass housing, as the name suggests, is for the masses. Most of the time, it is associated with social or public housing. In this research, mass housing can be either public housing supplied by the government, or private sector housing developed by private companies. The 'masses' refers to homebuyers who will buy the units to live in them to meet their own needs; not for financial return via rent. However, due to the evolution of housing ownership systems, the definition of 'homebuyers' could also include families who are renting the units long-term. This is especially true in Japan where the apartments belong to corporations. Further details are explained later, but in short, 'homebuyers' means the families or individuals who will be living in the units.

Here, 'mass housing' is associated more closely with the cooperative housing which has proven to be a more effective and socially-relevant way to provide housing units. This is housing provided by enterprises who aim to tackle housing problems by organising demand and combining small groups of homebuyers economically. This method provides negotiating power to individuals and reduces the final cost of housing by supplying to many. Successful examples can be seen around the world such as Redditch Co-operative Homes in the UK, an organisation which has created 400 homes spread around the town of Redditch, through a series of five neighbourhood co-operatives - each self-managed by residents. However, most cooperative housing comprises individual houses. Mass housing in this research focuses on high-rise apartments, developing a more collective, collaborative manner with homebuyers to meet their individual needs.

\subsubsection{NATIVE HOUSING}

Housing has evolved dramatically over time to accommodate various human needs. Tracing all the way back to the Indians of South America, Alfred Métraux wrote in his handbook (1946) 
that Guyanese or Amazonian Indians will design their hut based on the environment to facilitate comfort.

Although the vernacular element is still widely in practice today, what is interesting is that the hut of every Guyanese family is compartmentalised differently based on their role and needs.

\subsubsection{VILLA BUILDINGS}

Moving forward to urbanism, increased housing demand resulting from masses flocking to cities, required a rapid solution. This is when verticality and repetition began to take shape. There were concerns about putting families into 'concrete boxes'. One main issue was that gardens, common in individual housing, were impossible in apartments. In 1922 Le Corbusier offered 'villa buildings' where each apartment had a garden (Figure 2). However, these failed to attract the desired attention as the masses felt that the garden did not have anything (clouds, stars, birds, sky) above it except a concrete ceiling.

Villa Buildings demonstrated that vertical housing need not be just simple apartment units but can be as luxurious as a villa. Importantly, however the apartments were still repeated and unable to reflect the needs of individual families.

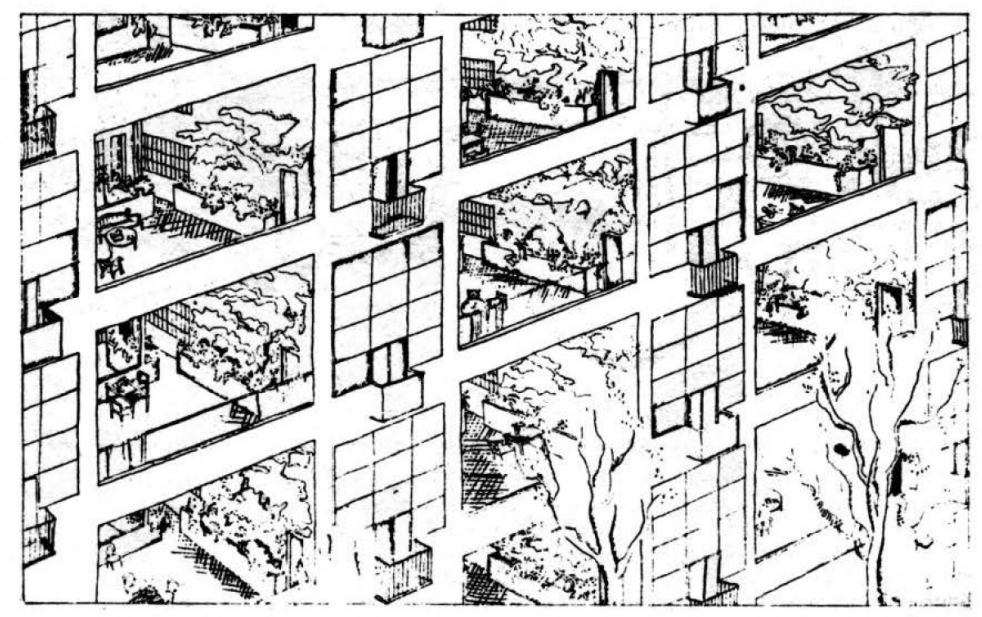

Figure 2 Villa-Building - every unit has its separate garden (Corbusier, 1923) 


\subsubsection{SCHRODER HOUSE}

This first sign of flexibility before Le Corbusier's 'Five Points for a modern Architecture' happened in Holland. In 1924, Rietveld was commissioned by Mrs Schroder to build a house in Utrecht for herself and her three children. The house later became the manifesto of the De Stijl movement. Named 'Schroder house', the play of sliding partitions on the second floor provide a flexible space for different functions at different occasions.

Although flexibility did not play a major role in characterising the De Stijl movement, integrating it in Schroder house, which is not an apartment, provides a leading example of non-structural moving walls providing spatial flexibility to adapt to the functional needs of the users in housing design.

\subsubsection{WEISSENHOFSIEDLUNG}

Then in 1927, in Stuttgart, a whole district of functional housing, named Weissenhofsiedlung, is built. It was a collective design project built by most of the later well-known architects for an exhibition. Mies van der Rohe was one of those architects and erected a set of apartments transformable by the inhabitants. It was a very avant-garde design given the possibilities of the plans.

This was one of the first projects of significance where a design intention was to make use of architectural components to allow design flexibility to meet the needs of different homebuyers. There are no records of how the homebuyers designed their space, but one thing is sure; the housing in Stuttgart offered the inhabitants semi-permanent partition of the space accordingly to their needs or desires. In exchange, the homebuyers had to accept the collective finishes of the building façade as shown in Figure 3. 

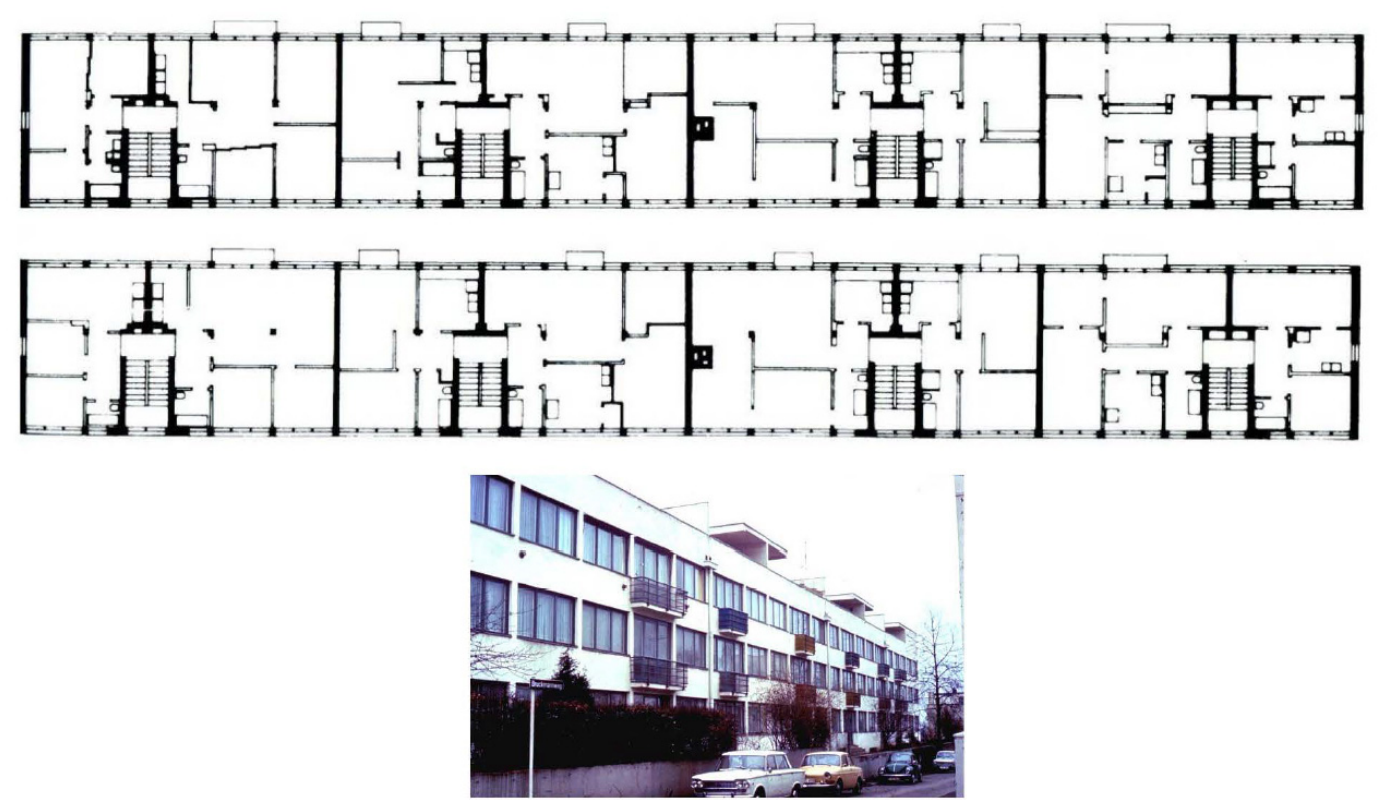

Figure 3 Flexible apartment of Mies Van der Rohe, Stuttgart, Weissenhofsiedlung (Top) Conventional Plans

(Middle) Free plan and continuous space (Bottom) Plain exterior facade (Arch+, Oct 1989)

\subsubsection{PLAN OBUS}

Although the progress so far indicates signs of flexibility and homebuyer-involvement, the next point of progress provides a greater picture of what a city might look like if the public is given the opportunity to participate. In 1931 - 1935, Le Corbusier drew a plan for the Algiers, named Plan 'Obus' where he envisioned apartments for the supposed quarter million newcomers into the city. Manfredo Tafuri was one of the few who analysed Plan Obus in depth and the observations in his book (Tafuri, 1976) very much apply to this research. Tafuri states that Le Corbusier's Algiers 'imposes a total involvement upon the public'. The main residential area in Plan Obus is underneath an elevated highway arching between cities. Those raw spaces, Le Corbusier believed, should 'little by little' be filled up by the people themselves. Le Corbusier also aimed for maximum flexibility, interchangeability and accommodation of rapid consumption. In his sketch (Figure 4), eccentric and eclectic elements are added to a network of fixed structures. Although this was sketched by Le Corbusier himself, it is possible to see how he anticipates the public expressing their own 'bad taste' design. In this example, every residential cell can be substituted or changed in some way to meet any individual necessity. 
This plan remained no more than a vision and became purely intellectual. Its 'failure' was in disregarding Algerian social and religious traditions, and the idea of allowing the people to shape the city was not what the government was then looking for. The design was too far ahead of its time, and to date, in fact, it remains unsurpassed from the point of view of ideology and form.

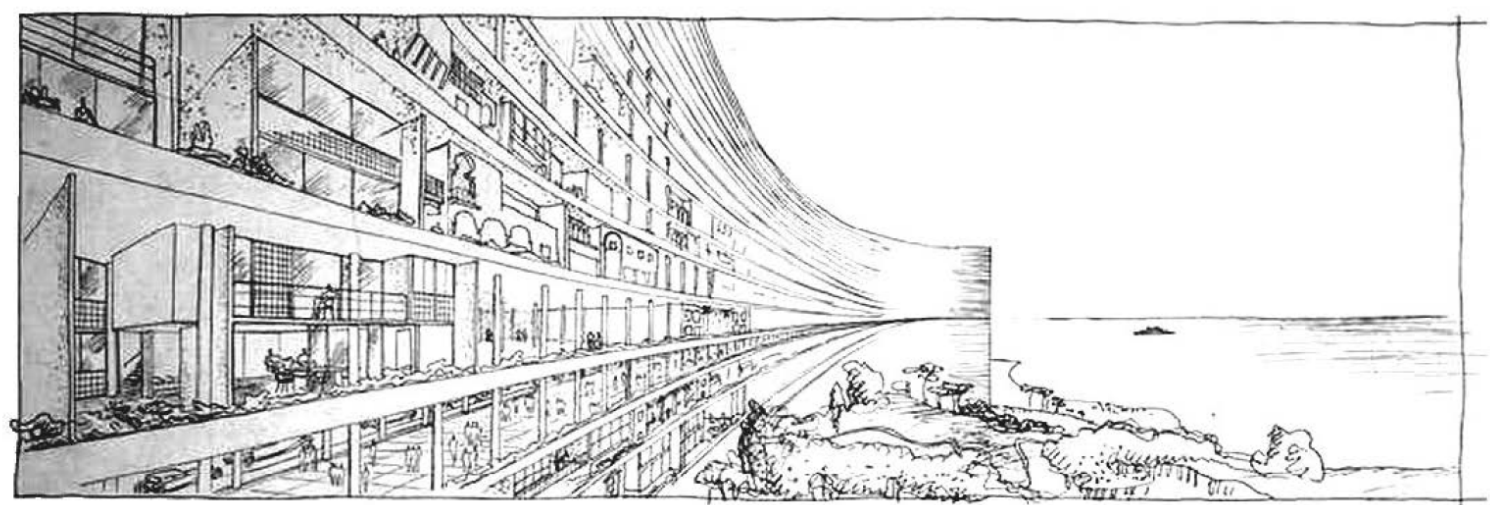

Figure 4 Plan Obus by Le Corbusier (Image taken on the web)

The legacy of Plan Obus was not lost. In the 1960s, John Habraken set up the Stichting Architecten Research (SAR) (Foundation of Architects Research) group in the Netherlands. The lack of variation and individual personalization in housing had initiated a new participatory paradigm in design and production, with the aim of allowing homebuyers' input into the design of their homes. While Le Corbusier, Walter Gropius, Frank Lloyd Wright, Buckminster Fuller, and Jean Prouvé were realising many works allowing homebuyers' input into the design of their individual homes, Habraken's work focused on multi-family housing. He developed the 'support' theory (Habraken, 1972) to establish the decisions over which the community has control. The 'support' is also a structure that is designed and built; comparable to the 'housing system' but without non-load bearing elements. The community are the homebuyers and the community decides 
how elements are placed within the 'support'. Although the Algiers plan notably influences Habraken, no SAR project provides a direct connection. Habraken's work plays a major role in involving homebuyers in the design of high-rise apartments - which is the focus of this research. The details of his concepts and methodology are further discussed in the next section.

\subsubsection{HABITAT 67}

The next 'evolution' happened in physical form. There was neither the participation of homebuyers nor flexibility of space. In 1967, Israeli-Canadian architect Moshe Safdie designed Habitat 67 (Figure 5), the Canadian Pavilion for the World Exposition.
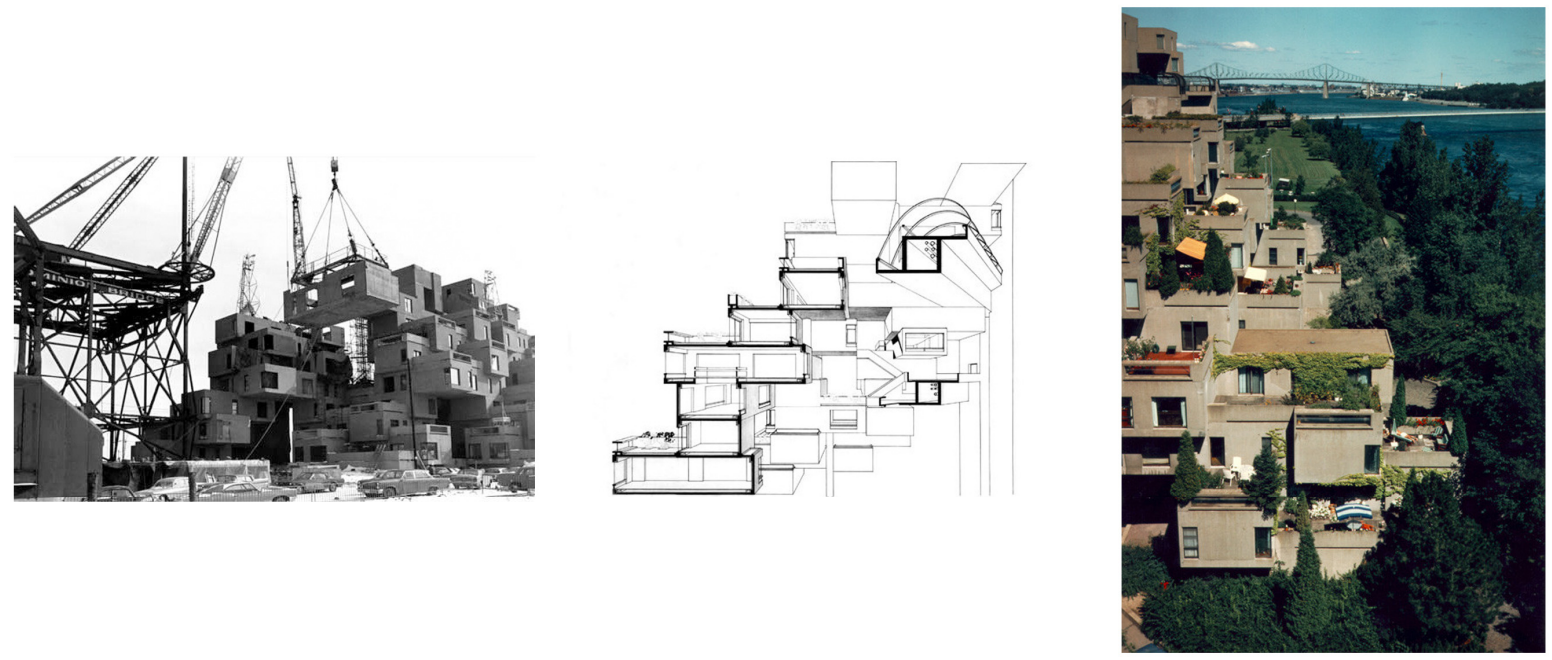

Figure 5 Habitat 67 by Moshe Safdie (Merin, 2013)

(Left) Prefabrication construction

(Middle) Section of Habitat 67

(Right) Bird-eye view image of Habitat 67

The project was originally Safdie's thesis, which he developed into a master plan for the world expo containing shopping centres, a school and 1000 housing units (Merin, 2013). The idea was to 'reinvent the apartment building', breaking out of the standard rectilinear building yet retaining the modularity for prefabrication construction techniques, potentially cutting the cost of the housing. Despite his great ambition, there are two sides of the story in the outcome. On the one hand, the project was reduced by the government to provide only 
158 units, causing construction costs to go over budget. The price of the housing was therefore increased tremendously to recoup these costs, to a level which no one could afford (Paiement, 2015). On the other hand, although Habitat 67 started off with a number of maintenance issues, its design remains a breakthrough even to this day.

Every apartment can be accessed via pedestrian street and bridges. By stacking the concrete 'boxes', every apartment has access to a roof garden with a constant flow of fresh air and maximum daylight. Looking back at Le Corbusier's villa-building, Safdie, in this Habitat, has achieved what Corbusier could not. Resident Jeannie Saunders mentioned in an article in 'The Guardian', that Habitat will always be "a community, where people have a feeling of friendship with neighbours, a special place to live". This is what this research aims to achieve more extensively.

\subsubsection{LA MÉMÉ}

The 1970s finally saw the idea of resident participation taken almost to full conclusion. It was a student housing project on the outskirts of Brussels (Figure 6).
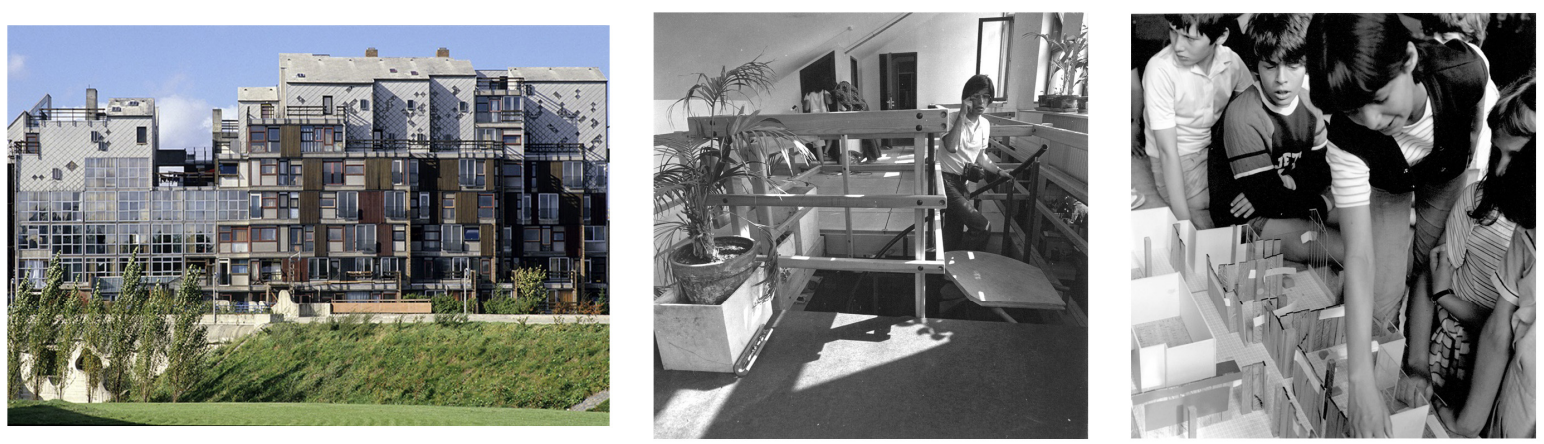

Figure 6 La Mémé by Lucien Kroll and the students (image by architect) (Left) Elevation of La Mémé showing different façade put together (Middle) Interior of La Mémé showing different size doors (Right) Design process by students

Due to some internal tension, the medical faculty of the Catholic University of Louvain had to be relocated. The students at that time protested what the university-selected architect proposed. They set up their own committee and produced a counterproposal by Lucien Kroll, 
an architect known for his co-operative manner of practising architecture (Graaf, 2016). Their choices approved, the students worked closely with Kroll for a period of two years in the privacy of his home. The location enabled their close collaboration and distanced them from the institution, allowing the necessary freedom to unfold a unique design process. Kroll pushed the collaboration to the extreme. Building only a shell house, he allowed the students who would be living in the housing to transform anything in it. Even when a tall American student decided to design a 7 meters tall but small room, neglecting the wishes of other students who would be the room's next inhabitants, Kroll remained silent. He gave the design decision totally to the students and was later proved right; that special room became a great attraction. The process was enjoyable until the school representative finally went to visit two years later. The university felt that the outcome, named La Mémé by the students, did not represent their 'values', and since it was also substantially over budget, Kroll was fired. Kroll, however, did not back down. He sought alternative suppliers and lowered material costs to fit the budget. He was determined to see the project to the end without making a single alteration to the community-led design.

This project shows that a bottom-up design process is simply responding to the needs of homebuyers. Although Kroll faced many obstacles with the authorities, the process also proved two things that this research is looking for: Flexibility could allow homebuyers to adjust their budget dynamically, and the design process could bring homebuyers closer together at the pre-occupancy stage.

\subsubsection{NEXT21}

So far, most of the design processes bringing about flexibility and involving homebuyers were initiated or envisioned by the architects. In Japan, there were even numerous efforts to develop the idea of a building system. In 1980, a national campaign took place to try to integrate all the various efforts into a single system and the Century Housing System (CHS) was formed 
(Schmidt, Eguchi, \& Austin, 2010). The primary aim was to extend the life of Japanese housebuilding by developing a systemic approach that allowed changeability of building components, increasing the adaptability of building functions. The system was based closely on the 'support' theory developed by Habraken; separating the building components into various layers based on durability and economic rationality. Initially, five layers (Figure 7) and eight points of synthesis were developed as a building guide. This was later simplified into just two levels (unchangeable 'support' and changeable 'infill').

\begin{tabular}{|l|c|c|}
\hline \multicolumn{2}{|c|}{ CHS 5 layer breakdown } \\
\hline \multicolumn{1}{|c|}{ Component Examples } & $\begin{array}{c}\text { Life span } \\
\text { category }\end{array}$ & average \\
\hline light bulbs, packing & $3-6$ & 4 \\
\hline hot water heater, home appliances, piping, wiring & $6-12$ & 8 \\
\hline movable partitions, built-in furniture & $12-25$ & 15 \\
\hline exterior door and windows, roof & $25-50$ & 30 \\
\hline foundation, main columns and beams & $50-100$ & 60 \\
\hline
\end{tabular}
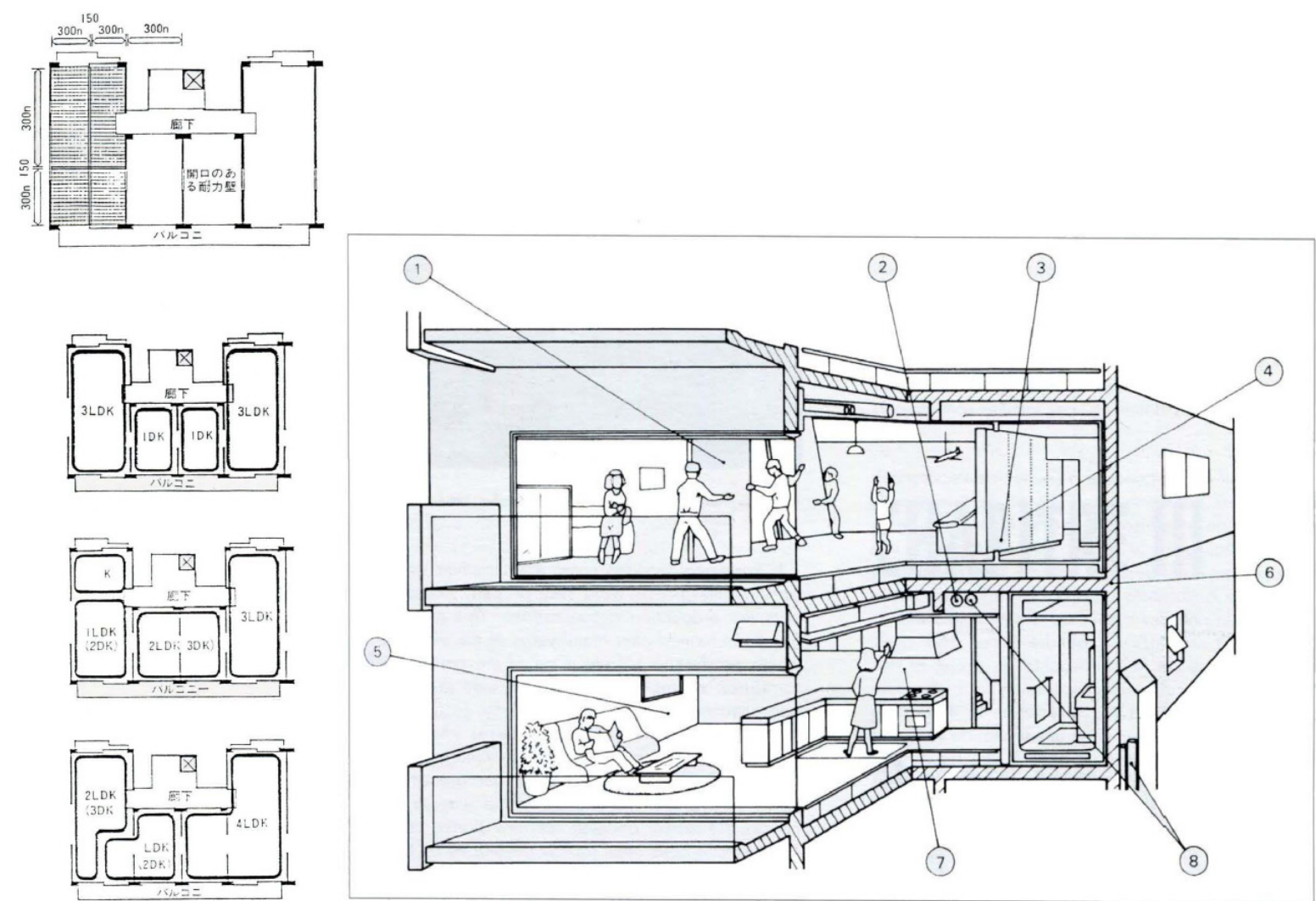

Figure 7 Application of flexible spaces with the five CHS layers and the eight-point synthesis (Center for better living, 1982) 
This SI (support/infill) system was later successfully implemented into the building industry and recognised by the whole of Japan. The system brought about a number of housing projects that provided flexibility for homebuyers. A ground-breaking example called 'Free Plan Rental Apartment House' was built in 1986 by the Japan Housing Corporation in Hikarigaoka, Nerima-Ku, Tokyo. Not only were designs separated into two levels, the ownership of each level was also separated. The building skeleton (Support) still belonged to the Corporation but the infill, including various facilities such as kitchen, bathroom and toilet, was totally owned by the homebuyers. This allowed the homebuyers to design the room layout freely, according to their needs and desires. Although the homebuyers were to pay several million yen for the infill, this allowed them to have better control of their budget. They also paid much cheaper rent (by fifty thousand yen or more per month) compared to other typical apartments (Seiichi, 2008).

In light of the government initiatives and many successful projects, a major city gas supplier, Osaka Gas, decided to develop an experimental, near-future-type apartment building and sought Professors Yositika Utida and Kazuo Tatsumi to develop the project (Seiichi, 2008). The building, Next21 (Figure 8), consists of 18 individual units designed by 13 different architects independently, and it has six floors with one basement. 

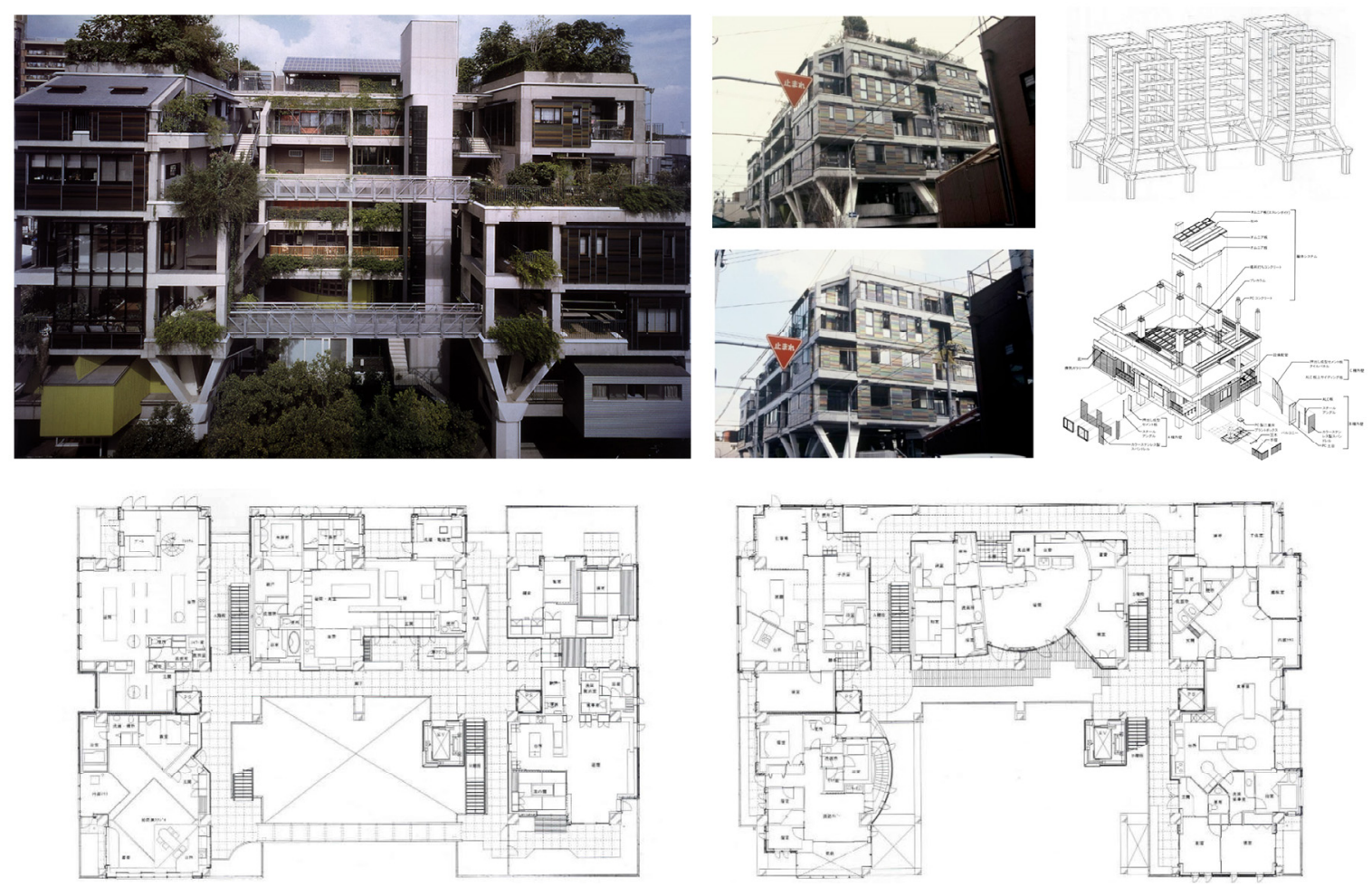

Figure 8 (Top-left) Front image of Next21

(Top-middle) Changes of façade

(Top-right) Separation of Support and Infill

(Bottom) Distinctive differences in floor plans (images by Osaka Gas)

For Next21, the focus is not solely on room layout. To inject character back into the cityscape of Japan, which lost their traditional beauty in World War II, Next21 also examines the elevation of the houses. Initially, too many varying designs by many different architects spoiled the overall design of the building. An external wall system was established to develop a sense of unity. The system follows a certain order based on material and colour, but still allows for freedom of arrangement based on a homebuyer's liking. It was a success, and another experiment was conducted to test the capability of changing the position of external walls.

Completed in October 1993, Next21 was opened to the public in April 1994 to begin its five-year occupancy experiment. Most of the first homebuyers were employees of the Osaka Gas Company and their families. User participation was an integral part of the design decisionmaking processes, and prospective homebuyers were interviewed to identify their needs and wants in the collective housing. There were indeed diverse needs recorded (Kim, Brouwer, \& 
Kearney, 2002) but the SI system, in addition to design modularity and coordination, provided the much-needed flexibility to fulfil every need of the homebuyers.

Next21 is one of the most comprehensive buildings that demonstrates and experiments with various design methods for flexibility of design, construction, operation and even maintenance. Its involvement of homebuyers was also well planned and coordinated. This is the one project which this research heavily references in a later chapter on developing a digital tool to enhance the participatory design process of mass housing.

\subsection{9 ÖKOHAUS}

Moving backwards a little, while Japan has developed CHS, another project is happening in Berlin. In 1987 the Ökohaus project (Eco-home), is conducted by Frei Otto and Herman Kendel for the International Building Exhibition (IBA). It is a collective housing which exercised, to a great extent, homebuyers' participation and open design. Frei Otto saw this as an opportunity to consider new ways of living in a high-density urban context. Frei Otto planned the design with reference to Le Corbusier's five points of architecture, which connects indirectly to Habraken's support theory (Danielsen, 1996). By providing only a two-storey concrete structure, Frei Otto allowed the homebuyers to do whatever they wanted for their houses with some 'ecological' restrictions. These included installing large bay windows for natural indoor sunlight, setting up green roofs and conserving the surrounding nature. The project started off as an article published in the local newspaper to attract potential homebuyers, or in this case 'co-builders and co-participants' (Lendt, 2012). There were many applicants, but several backed out after discovering the uncertainty of cost and time. In the end, 18 families were formed, and with the help of 9 architects (some were part of the 18 families), a community was formed to begin the design and construction of Ökohaus (Figure 9). 


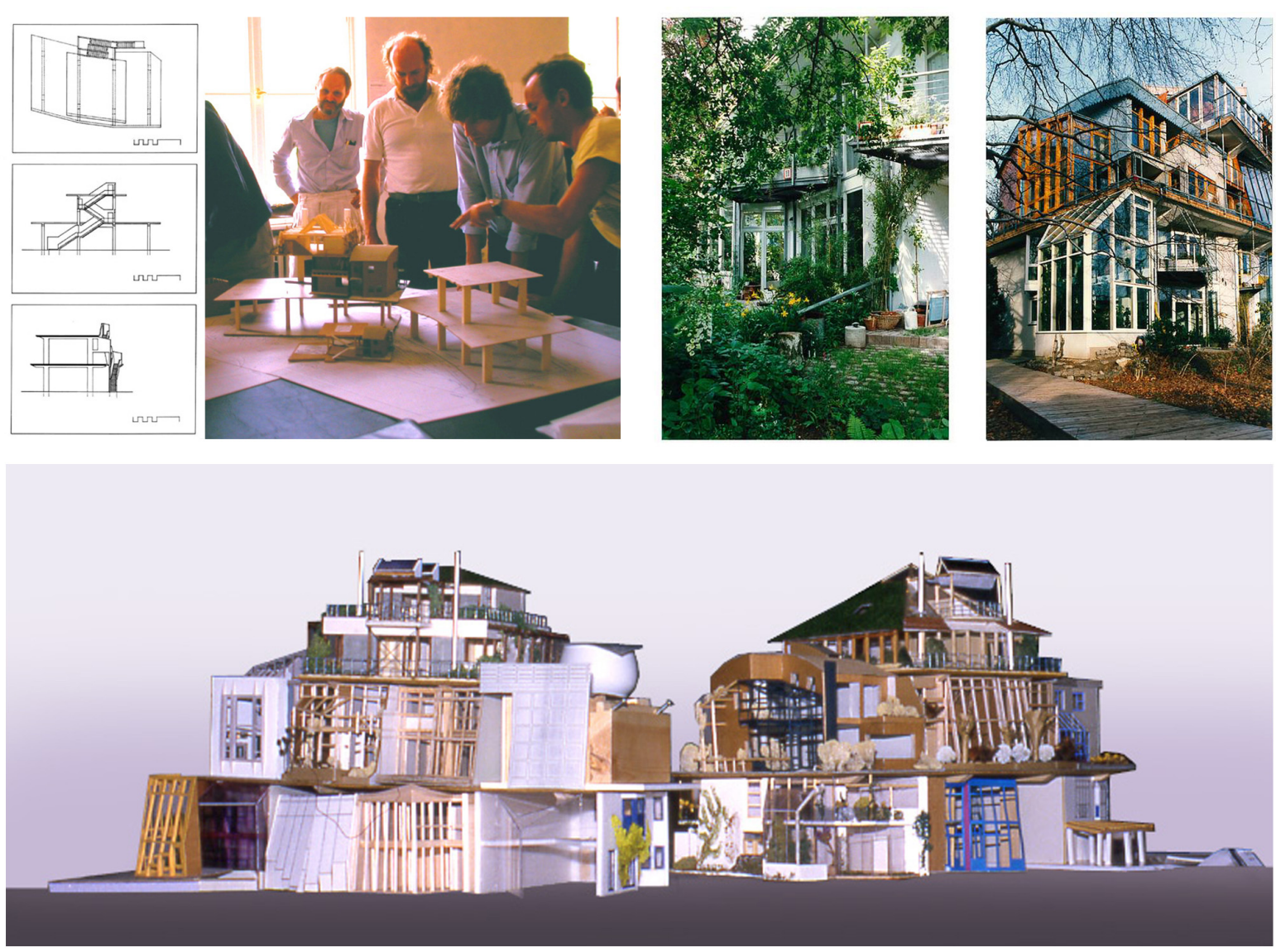

Figure 9 (Top-left) Structure drawing of Ökohaus

(Top-middle) Collaboration between homebuyers and architects using physical model of the structure (Top-right) Two images of the final constructed Ökohaus showing its integration with nature (Bottom) Digital model of the final outcome (sol•id•ar Architekten, 1999)

The design and planning stage took two years, and the construction took another two years. The community had an agreement that if one had financial problems, the rest would stand in to keep the project safe. It was a project with full devotion. Individual homebuyers had to invest more than they expected as the process faced a lot of obstacles. However, the outcome for most of the homebuyers was a success. Not only did they design and construct their house, they also formed a close bond with other homebuyers and some families stayed there for generations (Lendt, 2012).

\subsubsection{HAUTS PLATEAUX DE BÈGLES}

After the 'success' of Frei Otto's Ökohaus, there was almost 30 years (although Japan started their initiatives and Switzerland also began its series of projects (Beisi, 1995)) before a 
'successor'. In 2012, the deputy mayor Noël Mamère, of Bègles, France, decided to promote 'vertical subdivision' to reconcile individual houses with gardens and personalization of housing, flexibility and scalability to counter urban sprawl (Rougé, 2013). Influenced by Ökohaus in Germany and Next21 in Japan, the project, named Hauts Plateaux de Bègles, is designed by architect Christophe Hutin, who worked in partnership with the city of Bègles, Domofrance, a social housing landlord and the Lafarge group, a construction company. It has 21 houses that are flexible from $120 \mathrm{~m}^{2}$ to $220 \mathrm{~m}^{2}$, with a garden allocated at least $25 \%$ of the area and all implemented in a 'megastructure' support (Figure 10).
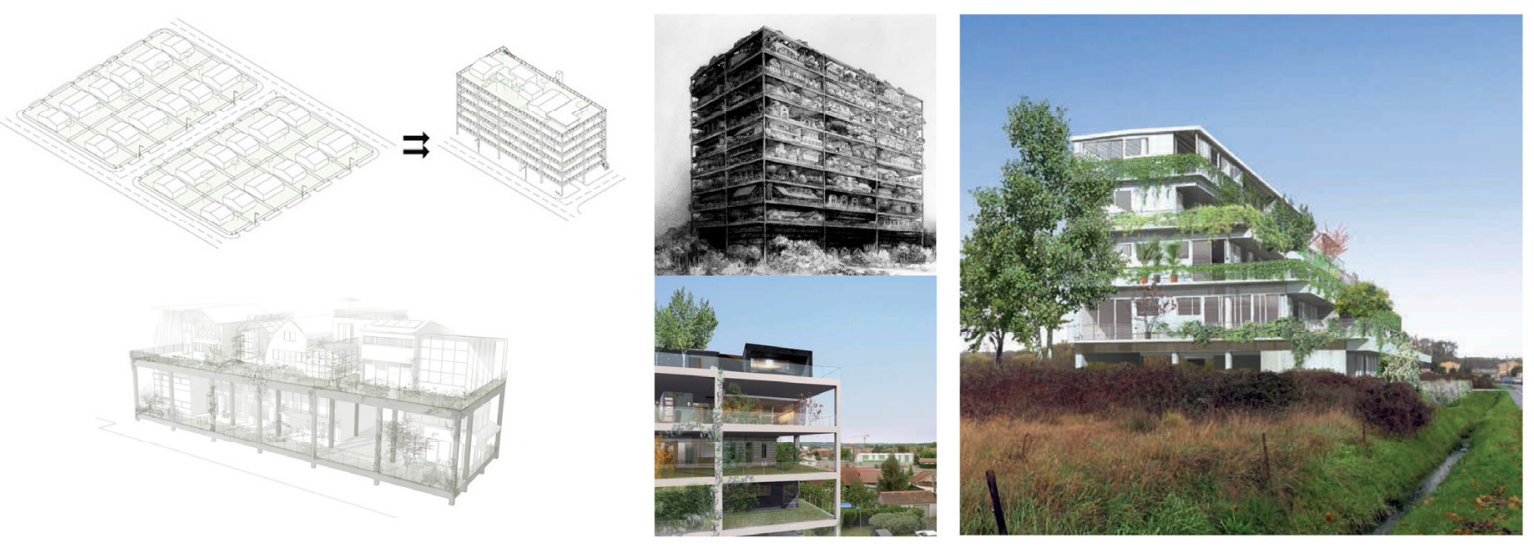

Figure 10 (Left) Concept and vision of Hauts Plateaux

(Middle) Comparision of outcome with image drawn by SITE

(Right) Final constructed Hauts Plateaux (images by architect)

Interestingly, the idea was very similar to the utopian project, 'Highrise of Homes', developed by the American agency SITE (Sculpture in the Environment). The difference is that in this example, the involvement of various stakeholders from politicians to economic actors made this concrete utopia real (Rougé, 2013). This innovation pushed the boundary of housing design and is the most current project that promotes flexibility and scalability in housing to answer to the different needs of homebuyers.

In summary, this brief background outlined the history of design evolution, each stage highlighting a valuable aspect. The early reflection of human needs in the Guyanese example; the vision of Corbusier's villa-building and Plan Obus; the first sign of flexibility in the Schroder 
house by Rietveld, and later in the Stuggart apartment by Mies; the ideology of support by Habraken; the form design by Moshe Safdie; the desire of the students in La Mémé with Lucien Kroll; the initiative of the Japanese government, which generated the system that shaped Next21; the multi-stakeholder collaboration in Ökohaus by Frei Otto, which inspired a more extensive collaboration in Hauts Plateaux de Bègles in France. The key designs, experiments and visions outlined here have contributed to the development of housing design that could involve homebuyers for their design inputs, understanding their needs to generate appropriate outcomes. At this stage, it is apparent that the capacity to involve homebuyers is there, and that advancements in the construction industry have already reached a stage suitable to support participatory design. It is thus easy to wonder why such housing design lost its appeal over time, and not taken up more generally. In the next section, this research looks into some more examples that were studied in depth with homebuyers, and that could provide further insights into the issues and ideas that could be adopted to encourage the involvement of homebuyers.

\subsection{Towards Flexibility, Participatory and Open Housing}

One of the most important tasks of architects in housing design, is the creation of space that reflects the desires and needs of the homebuyers. In order to do so, a 'bottom-up' approach is usually considered. The terms 'top-down' versus 'bottom-up' strategy is usually used by computer scientists to describe a way to approach programming design (Tutorialspoint, 2006). However, in architectural design, a top-down design is defined as an approach driven by a person or an organisation. The design will be almost totally influenced by that person's style and taste. On an organisational level such as a government, the design could be driven by directives. A bottom-up design is client-oriented, where clients' needs are the starting point of the design (Skipsey, 2015).

The initiatives of the architects outlined previously, demonstrate various ways of approaching tasks using a bottom-up strategy. However, to what extent do homebuyers demand a design methodology that enables their involvement in the design process? Various 
studies were carried out with homebuyers who stayed in those housing projects aimed at providing flexibility and enabling the involvement of the homebuyers.

\subsubsection{JÄRNBROTT}

In Sweden, architects seem to have been influenced greatly by the apartment designed by Mies van der Rohe in Weissenhofsiedlung, Stuttgart, 1927 (Krantz, 1976). The idea of flexibility was not an original objective. There was a shortage of skilled labour in Sweden, and it was necessary to reduce the amount of skilled labour required on site. The obvious step was to factory fabricate as many parts as possible, and thus detachable partitions and then the Skarne 66 system were formed (Pollit, 1981). In 1954, the first experimental housing towards adaptable collectivism was built in Järnbrott district by Tage and Olsson (Figure 11). It comprises 20 apartments on five floors with only two static elements: the bathroom and the kitchen. Everything else is removable, including cupboards that act as partitions at room height. A survey was done ten years after the building was built and occupied. The result is as follows (Krantz, 1976):

- There were 38 families living in the 20 -apartment building.

- The original living room of $18 \mathrm{~m}^{2}$ was changed by tenants and subsequently varied from $18 \mathrm{~m}^{2}$ to $37 \mathrm{~m}^{2}$.

- The function of the living room had changed to serve as a playroom for children more frequently, when in conventional Swedish traditional style apartments children's access to the living room was forbidden.

- Almost a quarter of the families carried out the transformation, and the most common reason was for the children in terms of need for surface space, storage or isolation. 

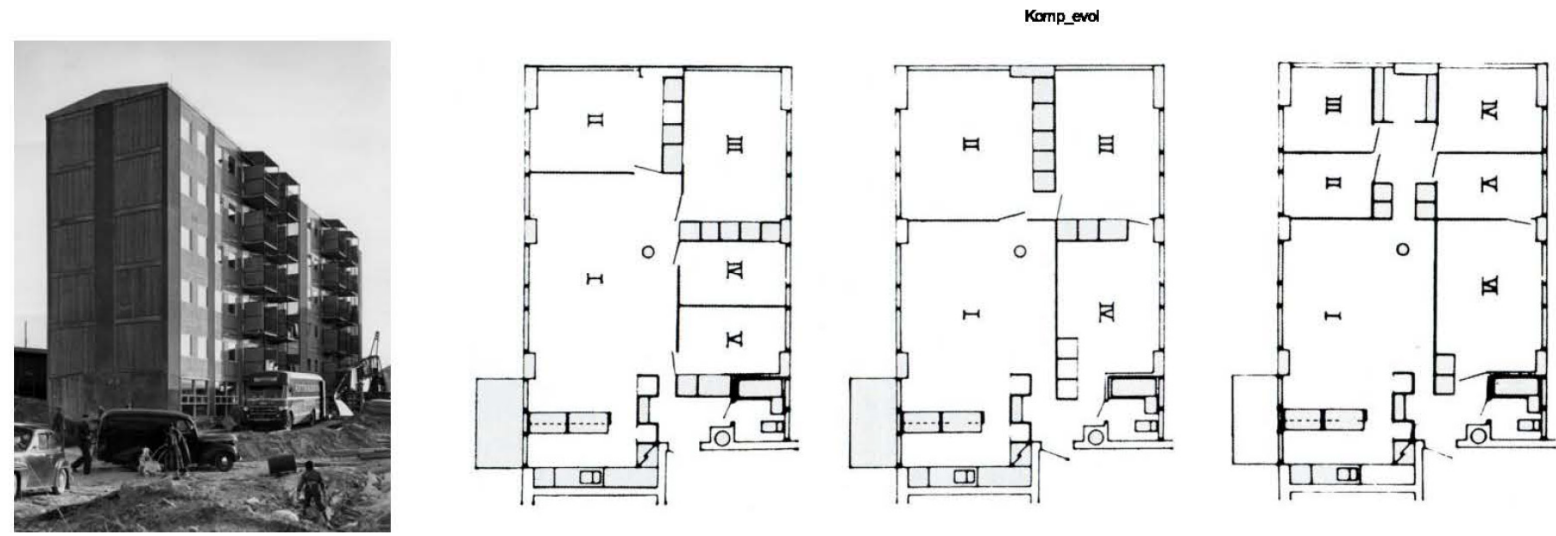

Figure 11 Building of the Järnbrott district, and three plans made by tenants (Krantz, 1976)

Then in 1966 another flexible building of 16 apartments, quite similar to Stuttgart (1927) and Järnbrott (1954), was built in Uppsala. The one difference is that this building used fabrication techniques. Two surveys were done, one after two years of use and another in 1971 (after five years). The following was recorded:

- The homebuyers find the changes of elements hard to manage when the assembly requires two people.

- Most of the families voluntarily chose this experimental apartment specifically for the potential to make changes.

- Half of the families had already made changes to their units; more than half of the remainder made clear their intention to do so later.

- Most changes diverge from the traditional Swedish plan.

The study of the projects showed that the living style and needs of people changes over time. It is necessary for housing design to accommodate the changing needs of the people. Instead of learning such needs through post-occupancy surveys, the better way will include the homebuyers in the design process. 


\subsubsection{ORMINGE-WEST}

The last and largest studied example in Sweden, also by the University of Lund (Krantz, 1976), is Orminge-West, a group of residential units in Stockholm. It comprises 2600 units, of which 550 are flexible units. The study was carried out throughout the occupancy period (1967-1971) with the following recorded:

- There were ten plans proposed to the families, but none were taken. Also, the final layouts (Figure 12) were quite close to the proposed plan; being more open and having the kitchen incorporated into the living room. Children also had small individual rooms separate from the parents' room.

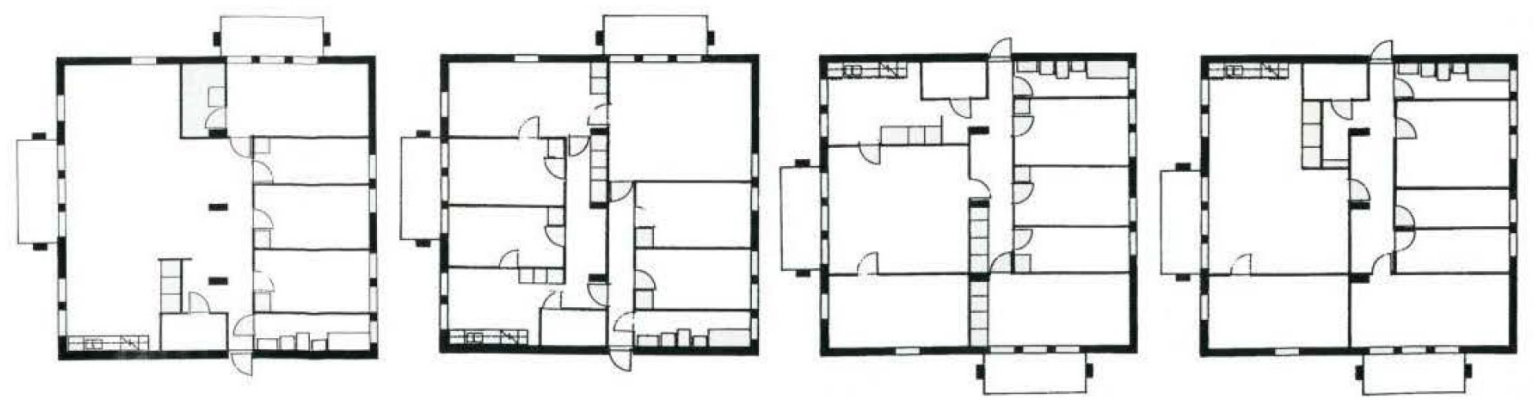

Figure 12 Diverse plans of Stockholm: Orminge-West, 1967-1971 (Krantz, 1976)

- The diversity of housing situations was considerable. Each variant of housing is inhabited by a different type of family, and similar families also adopted different housing solutions.

- Although preference for flexibility only came third place, the most satisfied families were those who utilised this capability often.

- Social contacts between neighbours seemed to be more frequent among the families of flexible units to talk about their unique space and housing qualities.

- The families of flexible units did not only transform their units but even began to take initiatives to make changes to the environment.

- The architect who designed the housing believed that the plans created by the families were better than those he designed himself. 
The three studies done by Birgit Krantz and the University of Lund showed that homebuyers do have the desire to make changes to their living spaces to suit their needs. The advantages go beyond spatial advantage. With the activities of the homebuyers and their creativity seemingly proportional to the opportunities provided for them to be involved in the creation of the environment, it seems that flexibility could also change the social aspect between homebuyers.

\subsubsection{MONTEREAU-SUVILLE}

However, although such participation in housing design worked in Sweden, the same applications did not work out quite as satisfactorily in France. As early as the 1950s, PaulHenry Chombart de Lauwe, an urban sociologist, stressed the need for new experiments in living accommodation to improve housing conditions (Periáñez, 1972). Montereau-Suville, designed by Arsène-Henry and Bernard Schoeller, was accepted by Motereau HLM (Habitation à Loyer Modéré) as an experimental building and was also the first to be completed in France. The building is designed as a simple square plan separated into four units per level (Figure 13). A unique feature of this building is the accessible terrace on the tenth floor being made into a collective residential area of kitchen and bathroom. Another is the gridding of the plan to aid the design process. 

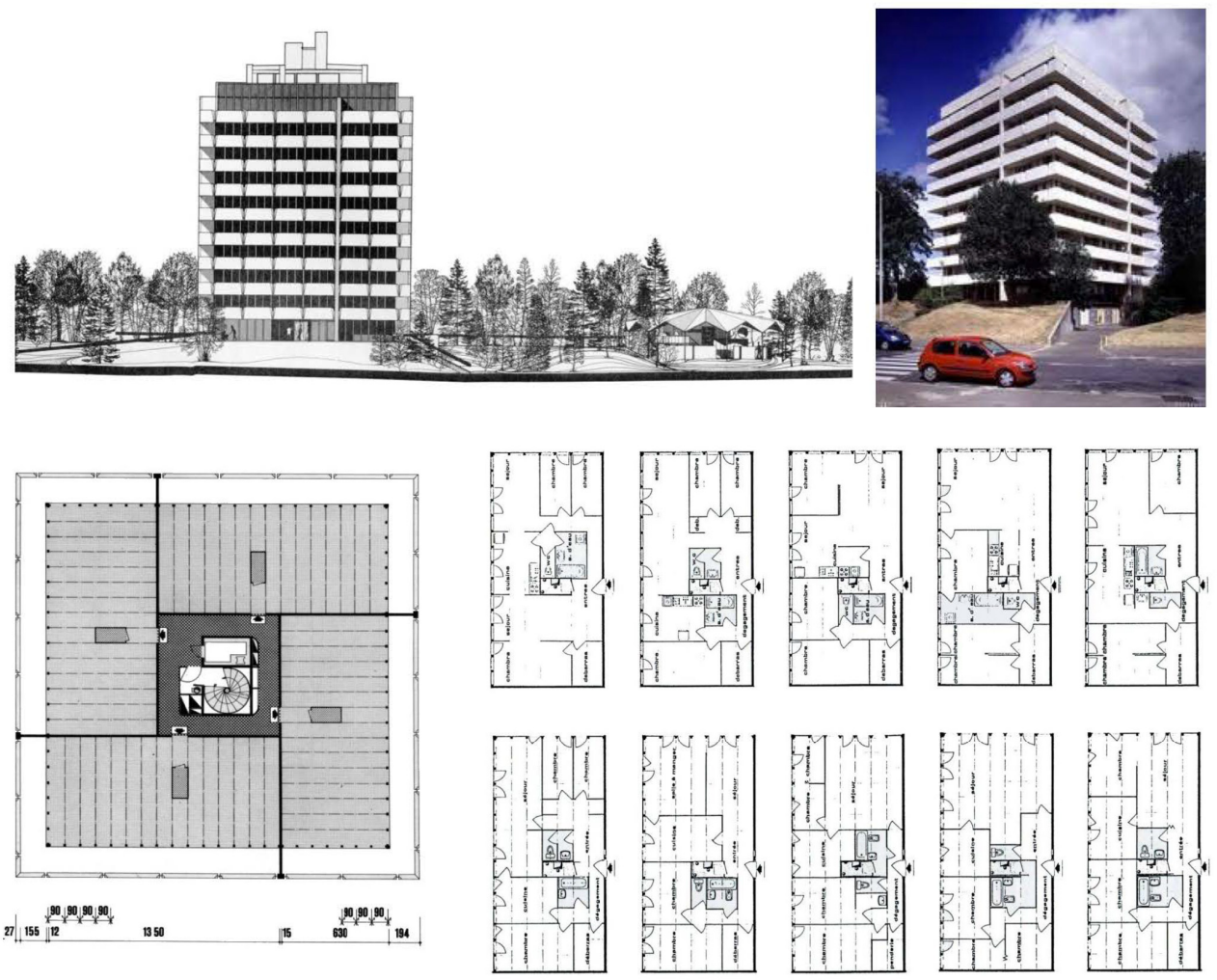

Figure 13 (Top) Drawing and image of Montereau-Suville (Bottom-left) Plan of Montereau-Suville separated into four plots (Bottom-right) Unit plans by architects(top) and homebuyers(bottom) (Periáñez, 1972)

Communication between the architects and the homebuyers was very 'directive-driven' and based on the Office of Sociology Research of the Ministry of Housing. This was to avoid hesitation by the homebuyers in making decisions. The first two years of occupation of the Montereau building saw its peak in performance where the homebuyers used the terrace residential area for various activities and friendly relationships between families were formed. Five years later, however, the euphoria dropped when the terrace area was locked due to degradation. The flexibility came at quite a cost, and homebuyers left to pay more than they could afford - though they were willing to do that initially (Pollit, 1981). In addition, the authority was not pleased with the weight of management. They were concerned with spending to revert the units back to standard when families moved out (this was later disproven as new homebuyers could live in the units without any difficulty and make changes if necessary). Quite differently from the Swedish cases where most changes happened in small units, the French only observed a change of plans in larger units of about $27 \mathrm{~m}^{2}$ per person (Periáñez, 1972). 
This project shows that similar intention to include the homebuyers can generate different results if the apartment building is not maintained well and managed accordingly. Also, different countries might have different needs which is an important factor to consider during the design process.

\subsubsection{ROUEN-LA-GRAND MARE}

Around the same period (1969-1972), another building, Rouen-la-Grand Mare (Figure 14), was being realised by Jean-Philippe Rameau Group. This was more of an architectural and technical innovation than flexible housing for homebuyers. The project was the result of the work of GEAI (a research group for industrialised architecture), to allow a great deal of freedom in partitioning, reassignment of space and modifiable façade. The building brought glass and aluminium manufacturers together with the steel sector, and was prefabricated and assembled on site in sixty days (BMC2, 2016).

A study on flexible housing was done by Raymond Fichelet, who interviewed the families staying in the building. His study found that homebuyers were not informed about the flexibility of the units. Although glazed windows provided natural light and entrance halls were large, new homebuyers of the time were unused to this design and complained about the soundproofing and heating of the wooden partitions. Moreover, one interviewed family member exclaimed fear after realising that all partitions could be removed (Fichelet \& Fichelet, 1973). The construction materials proved a poor choice, with fire breaking out three times in a decade. Yet the families rallied to defend the buildings from being demolished near the end of the 1990s. Although the project was poorly executed, the community bond was strong. 

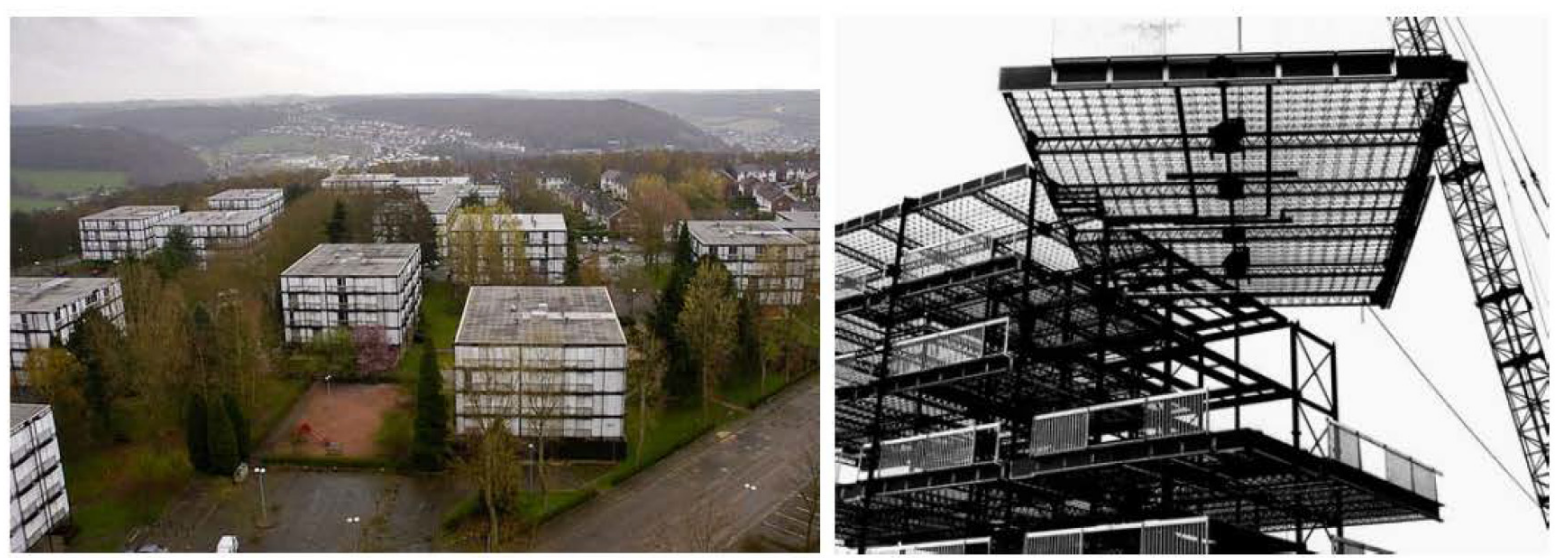

Figure 14 Rouen La Grand Mare and the GEAl process (Fichelet \& Fichelet, 1973)

This study did not demonstrate much about flexibility and the participation of homebuyers. The project does, however, demonstrate the importance of management and design within design methodologies of this type. Communication between the authority and the homebuyers must be clear to ensure sure this kind of project is targeted to the right type of homebuyer. The next example presents management failure of a different kind.

\subsubsection{LES MARELLES}

Les Marelles in Yerres (1971-1975) was experimental housing by architects B. Kohn and G. Maurios. The design and development of the project were very well planned and broken down into three ambitious components (Maurios \& Herrou, 1976):

Technological objectives towards 'total flexibility.'

- Construct a 'support structure' that can input independent subsystems

- Allow homebuyers to arrange the subsystems at their own will

- The structure can be broken down into modular, industrialised, precast concrete elements

- The structural elements can be assembled as a 'meccano', without the need for additional components. 
Sociological objectives towards greater motivation of the homebuyers:

- Homebuyers can design their own housing plan

- The total area can be divided flexibly, according to the needs and constraints of the homebuyers

- The units can be enlarged or shrunk by the homebuyers, with neighbours' agreement

- Flexible operations can be renewed over time without technical constraints

- Motivations of homebuyers should be observed throughout the housing design process

Research hypotheses with rigorous protocols, 1:10 model, video recorder, life-size simulations of the desired spaces:

- Is the process of self-design of the plan based on any essential psychological factors?

- Is the spatial relationship based on hierarchy dominance, conflict, understanding, etc. between family members?

- Design assistant provided by a psychologist whose role is to facilitate the development of the housing

With such a well-prepared scheme drawn out, one could easily imagine a success with a long lasting impact on future works of a similar genre. However, this project, which could accommodate 100 units, only had 15 buyers (6 withdrew afterwards) who benefited from the design process and drew their own apartment plans (Figure 15). 

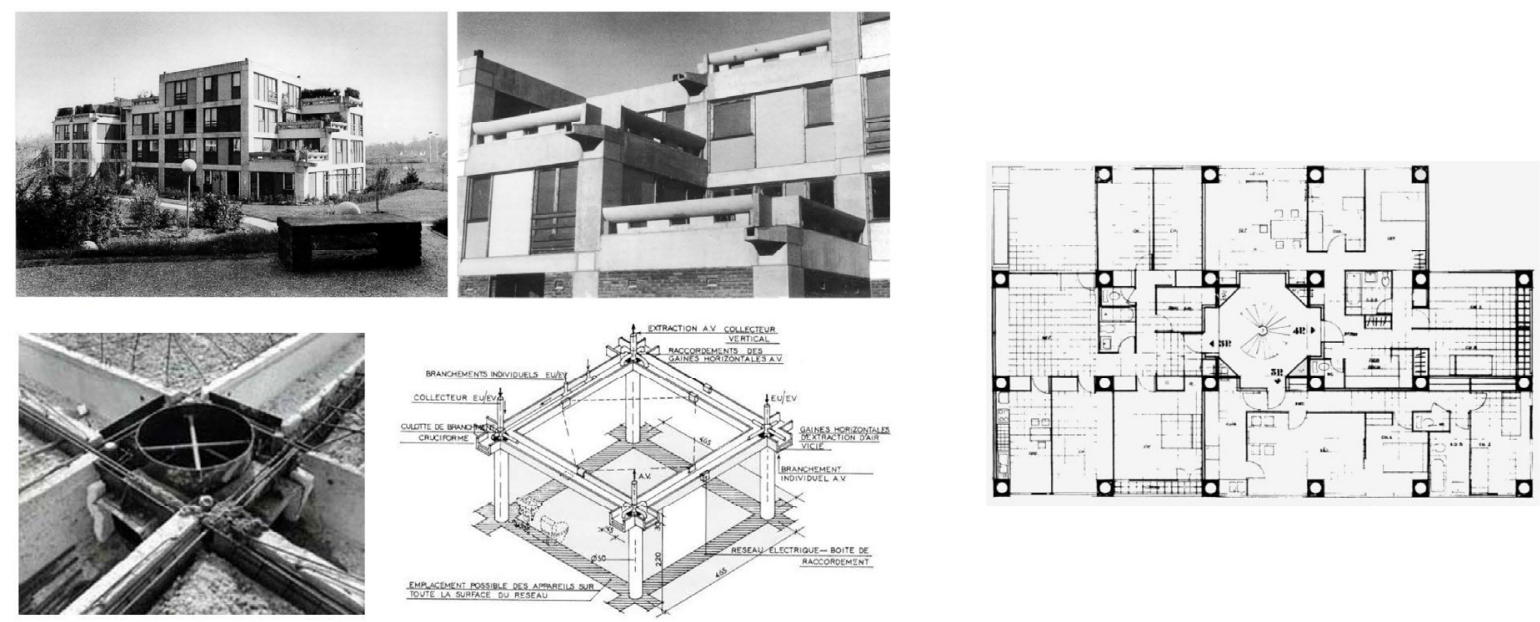

Figure 15 Les Marelles in Yerres (1971-1975)

(Left-top) Overview and roof detail of Les Marelles

(Left-bottom) Image and drawing detail of utilities embedded in structure

(Right) Floor plan designed by homebuyers (Maurios \& Herrou, 1976)

After a year, the experiment was terminated prematurely and the following explanation given (Maurios \& Herrou, 1976):

- The flow of funds was blocked

- There was no advertising outside of Val d'Yerres where the project is situated

- The press failed to promote the experience

- Reception for homebuyers during the whole process was badly prepared

- Design was chaotic with a lack of green space

- Delivery of apartments was delayed

It seems that although Les Marelles incorporated the teachings of the previous two French experiments, it was not well-executed and failed commercially. The failure happened exactly at the point where the decision-making was given to the homebuyers. At a management level, managers assumed that since the design 'power' was given to the homebuyers, they need do nothing; everything would be 'self-managed'. If the design 
works, managers need not commit any effort, and where it fails they can make use of the 'directive' measures to resolve it (Maurios \& Herrou, 1976) This approach caused the project to end in disaster.

The examples in France demonstrate that if a project is not managed well, the potential will not be realised. Even with homeowners who have a strong desire to design their own living space, the wrong approach can easily cause negative results. In fact, it is proven later that the design process demands more than just design desires and managerial preparation to ensure a smooth execution (Beisi, 1995).

\subsubsection{JOHN HABRAKEN}

Projects around the world involving the idea of flexibility and the participation of homebuyers were, to a great extent, influenced by Habraken, and his idea of the support structure and industrialised detachable units. It is therefore necessary to look deeper into Habraken's concept and gain an understanding of its capabilities and limitations.

John Habraken is a Dutch architect whose main interest is in mass housing and strategies for the participation of users and residents in the building process (Spatial Agency, n.d.). In 1962, he published the book 'De Dragers en de Mensen', later translated into English as 'Supports: an alternative to mass housing', and set up the SAR group to further his research. His book questions the nature of residential architecture built for people who are specifically excluded from making basic decisions about their living environment. He advocates against the idea of a house as a repetitive, finished consumer product; rather that it is a progressive relationship between people and their environment, based on the act of their daily lives. He proposed an alternative to housing design by separating it into two distinct components; the support and the infill. The 'support' being the building structure or 'base building', the design of which is based strictly on regulation and usually performed in a top-down manner. The 'infill' is the transformable components such as interior divisions and furniture. This, however, is only the technical aspect. The most important idea for which Habraken advocates is for homebuyers 
to take responsibility for their living environment; to be able to make autonomous dwelling decisions on their own behalf, rather than simply being provided with one (Habraken, 1999). For this to happen, flexibility is necessary, requiring his two-level techniques. At the same time, the support means more than just the building structure. It includes the setting up of a framework to support a bottom-up adaptive process that takes place throughout the habitation of the homebuyers.

It seems that projects such as Les Marelles took Habraken's idea too literally, providing only the technical structure and planning as support and expecting homebuyers to generate the final outcome themselves. In fact, Habraken's idea accounts for the decision-making process. By separating the building framework, he divides the responsibility and decisionmaking between administrators and homebuyers respectively. However, that does not mean that the administrators are free of other responsibilities. In Habraken's view, he is not creating a new type of housing architecture; he is just hoping to change the way architects design housing and their role in it; to take a step back and collaborate with the inhabitants collectively, using an open-ended architectural framework that could change and adapt over time. This is especially true for large residential buildings, for which a design by just one architect becomes a 'ludicrous stance' (Habraken, 2001).

Although Habraken was the most influential person with regards to flexible housing and the involvement of homebuyers, he was not the only person coming up with alternative ways of thinking about designing the built environment. After World War II, Europe was going through a major housing crisis. Most planning policies were repetitive, massive and homogeneous to alleviate the housing shortage. Policies that Jane Jacobs stated were destroying inner-city communities by creating dysfunctional settings that ignored local details and human-centred considerations in favour of the 'big picture' envisioned by master planners and architects (Nabian \& Ratti, 2012). This inspired the emergence of various other ideas to counter the modernist planning, including Bernard Rudofsky's 1965 exhibition, Architecture Without Architects, at the Museum of Modern Art. It is accompanied by a book (Rudofsky, 1964) to 
provide a unique view into what Rudofsky defined as 'communal vernacular', 'anonymous', 'spontaneous', 'indigenous', 'rural', 'non-formal', 'non-classified', or 'non-pedigreed' architecture. Moreover, Christopher Alexander's book, 'A Pattern Language', provided an 'open source' methodology involving the use of 253 architectural 'patterns' which he defined to enable people a say in designing their own environment. Each pattern describes an environmental problem and its core solution. The patterns could be mixed and matched, adjusted and supplemented as necessary, giving a high degree of design freedom to the people with its infinite potential combinations of multi-layered problems and corresponding multi-layered solutions (Alexander, et al., 1977).

Housing flexibility became a trend for a period later known as Metabolist movement, with the appearance of the iconic Nagakin Capsule Tower by Kisho Kurokawa in Nagakin, Japan (currently facing demolition) and eventually with the formation of Archigram. Although the ideas behind these examples provided an inspiring approach and methodology for addressing the shortcoming of modernism's top-down, authority-controlled paradigms of city building; they failed to revolutionise the way cities were built. Most of the examples are one-off experimental projects. In Sweden, the notable examples left behind only observational studies without any successors. Flexibility, on the one hand, was criticised as propagating a 'false neutrality'; often considered an ideological myth or merely an architectural toy (Till \& Schneider, 2005). James Stirling even declared that he was 'sick and tired of the boring, meaningless, non-committed, faceless flexibility and open-endedness of the present architecture' (Forty, 2000). Although his comment was to justify his design for the Stuttgart Staatsgalerie in the early 1980s, it brought about widespread concern that the concept of flexibility had outgrown its ability to deliver. Of the participation of homebuyers, Christopher Alexander in his Oregon Experiment said that "Participation will create chaos" (Alexander, 1975, p. 45). Moreover, in the Nature of Order he wrote, "the idea of involving users in the discussion, hearing their conflicting visions, trying to reconcile opposing points of view can become a political and administrative nightmare" (Alexander, 2002, p. 261). 


\subsubsection{OPEN BUILDING}

It was only when Habraken's idea was subsequently developed into an approach generally known as 'Open Building' (OB) (Kendall \& Teicher, 2000), that the core of his idea was interpreted more clearly and better applied. The basic tenets of flexibility and involving people with housing design remained, and the whole idea of support and infill further extended to environmental levels, encompassing the organisation of processes from a district level, through to the detail level of furniture in unit layouts. This breaking down of specific levels (Figure 16) acts as a principle tool for incorporating the principles of $O B$ into building design.

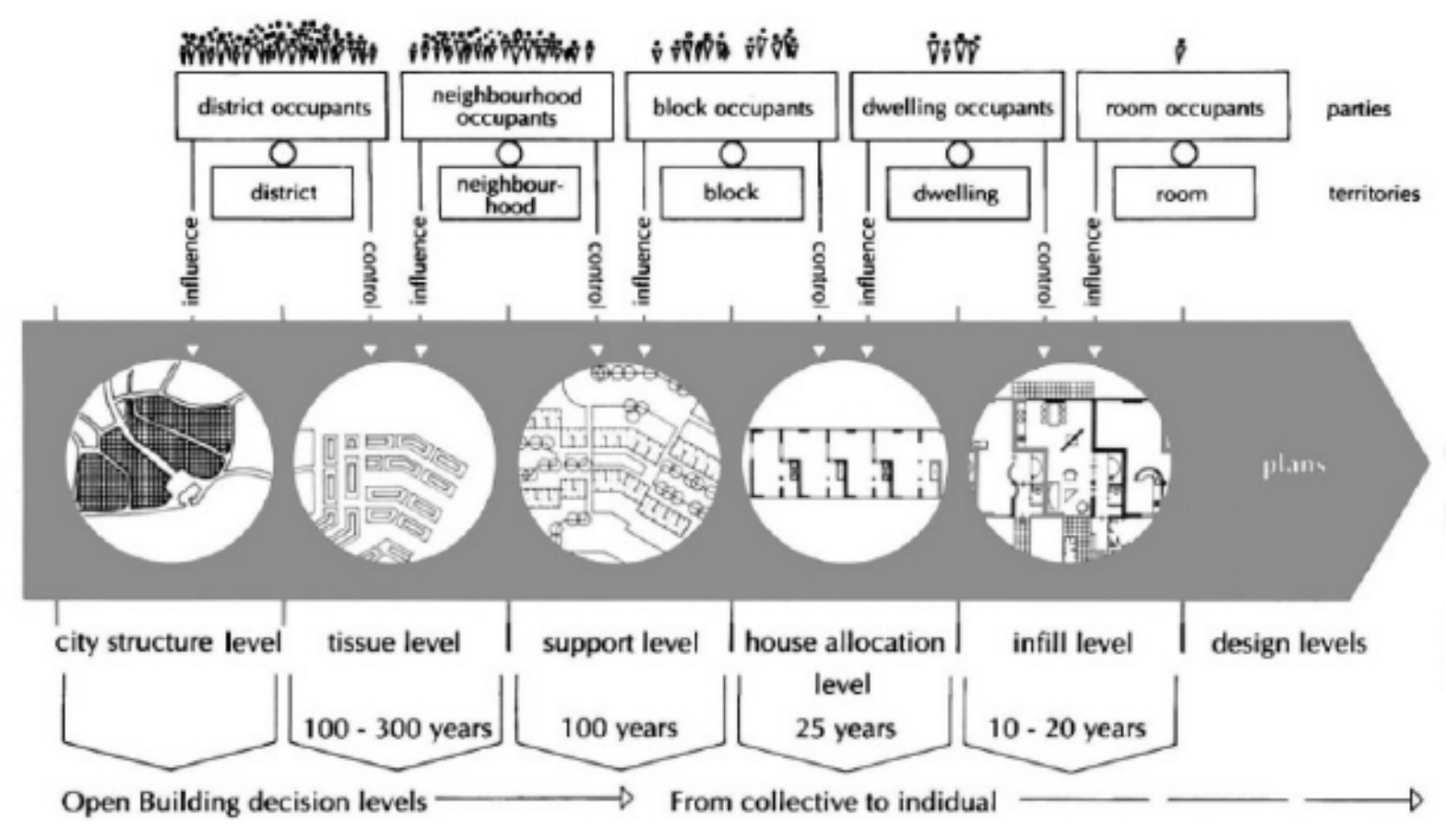

Figure 16 Diagram of environmental levels in Open Building (Kendall S. , 2004)

The application of $\mathrm{OB}$ is not restricted to residential building. In fact, the idea of environmental levels is not new, but a clearly formulated structure of these is. The structure is based on Habraken's 'The Structure of the Ordinary: Form and Control in the Built Environment' (Habraken, 1998). Each level is separate, yet dependent. The town fabric (tissue level) is of a higher level than the buildings, positioned within the town fabric. Buildings can be altered or replaced, while the town fabric remains consistent. The buildings, in turn, can be divided into the base building (support level) and the fit-out (infill level). The higher level (support) 
accommodates and limits the lower level (infill), which in turn determines its requirements towards the higher (Cuperus, 2001). On every level, there is an 'ultimate customer': the consumer on the infill level, the housing corporation or developer on the support level, the municipality on the tissue level. The idea of OB can be more easily identified based on these factors (Kendall \& Teicher, 2000):

- Recognising and organising the project according to the levels

- Decision-making is distributed

- Separating the environmental level physically

- Putting disentangling subsystems into buildings

- Structuring the professional service to support the decision-making process

- Using specific OB methodological tools

- Using support technologies with infill systems

- Using specific infill technologies

- Using $O B$ financial instruments

Moreover, according to Habraken, $\mathrm{OB}$ is used to indicate the various different yet related ideas about making the environment in general (Kendall S. , 2004):

- The level of 'intervention' to the built environment represented by 'base building' and 'fitout' or urban design and architecture respectively

- The design outcome involves the design decisions made by users / homebuyers with the professionals

- The design process that involved multiple participants with the various stakeholders of the project

- The overall system that can allow one subsystem to be replaced independently by another of the same function 
- The recognition and understanding of the building environment as being in constant transformation and change

- The acknowledgement that the built environment is a 'product' of a forever-ongoing design process which is being changed part by part

The concept of OB has since been applied around the world at every level, especially in the urban context (Kendall \& Teicher, 2000). Although the application is not yet extensive in the context of residential building, the change in global trends is slowly seeing a need for just such a design method.

\subsection{Present Shifting Housing Inclination}

In contemporary urban centres, population growth has transformed housing into a high-rise mass housing typology where individual families live in apartment units. To accommodate increasing demand, apartment blocks have been simplified to mass-produced 'containers' for shelter and living. Such models, however, lack individuality; a crucial quality for successful housing developments. Individuality is commonly held to encompass varying factors of value and need; factors that differ widely among sociodemographic groups. These can have a remarkable impact on spatial requirements. According to recent reports by the Organization for Economic Co-operation and Development (OECD, 2016), household structure in modern society is changing, and the typical modern 'two parents, two children' family becoming less common. This results in changing patterns of family living, education, work, entertainment and technology.

With globalisation, variations in lifestyle and household structure are seen most clearly in developed cities. This results in mass housing designs ineffective for multi-faceted social needs; 'forcing' people to live in identical units designed and built on concepts of mass production for efficiency and affordability. Today, as a result of new socio-economic realities, homebuyers are becoming more selective in their demand for change. The "one model fits all" approach seems to have run its course (Kieran \& Timbrelake, 2004). In response, a new 
strategy providing resilience for high-rise housing is necessary. While the viability of mass production techniques fluctuated in other economic sectors over time, in the 1970s a sharp increase in demand for personalised goods and products contributed to the call for a new production model.

However, the spatial categories of apartments remain at only studio and 3-room to 5room apartments (in Asia context). In the conventional design of high-rise mass housing, developers base plans on their experience and market analysis (Gao et al., 2015). They then proceed with design based on their visualisation and realise it in a profitable and cost-effective manner. To provide efficient and affordable housing, modular systems and fabrication techniques are adopted. Developers and architects even developed standards to further enhance the efficiency and fitness of housing products. The housing industry is still in such an architect-oriented operating state that it is hard for occupants to have any means of participating in the design process to request what they want or need.

Ironically, if we look back to the Indians of South America, their purposeful hut served a greater function. Elsewhere,traditional canal houses, such as the one in Amsterdam, had a distinctive typology but each family managed their own house independently with the ability to change every detail of the house to suit their own needs and desires (Kendall \& Teicher, 2000). Although solving housing demands is still an issue, standardisation in the pre-war period was inevitable; because of time constraints, and the lack of a skilled workforce. It is hard to understand why, with the current advancement of technology and skills and the many successful examples around the world, is housing becoming more of a mass-produced, consumer product?

One argument is that homebuyers are incapable of planning (Friedman Y. , 2016). It may be true that in the past a layperson might not be able to plan for themselves, but with the present abundance of information readily accessible through the Internet and social media, it is easy grasp a basic knowledge of the process. One great example of a total bottom-up development is Torre David, a 45-storey office tower in Caracas, Venezuela. It was designed 
by a Venezuelan architect, Enrique Gómez, but abandoned before completion with the death of its developer in 1993 and the collapse of the Venezuelan economy in 1994 which caused the country to go bankrupt (Brillembourg \& Klumpner, 2012). Although the building was not finished, the main structure and floor plates were. The economic crisis also created many homeless families during that period and in 2007 Torre David (Figure 17) became an informal, improvised vertical home for such families. At present, it accommodates a community of 750 families. Without any support or control from the government, the whole occupation process was self-initiated by families. Internal walls were laid with cheap bricks. Living at higher levels is very inconvenient as families must climb a long flight of stairs every day. Stores were opened at specific floors to cater high-level living families as there are no elevators. In 2012, Urban Think Tank led by Alfredo Brillembourg \& Hubert Klumpner began to work with the community to improve conditions and understand the whole bottom up process. However, this was not what the government desired and in 2014, Torre David was transformed into a commercial centre and office tower, and the residents were relocated. This is an extreme example of the integration of formal structure and informal adaptation (Urban ThinkTank, 2015) and demonstrates the capability of a bottom-up development.
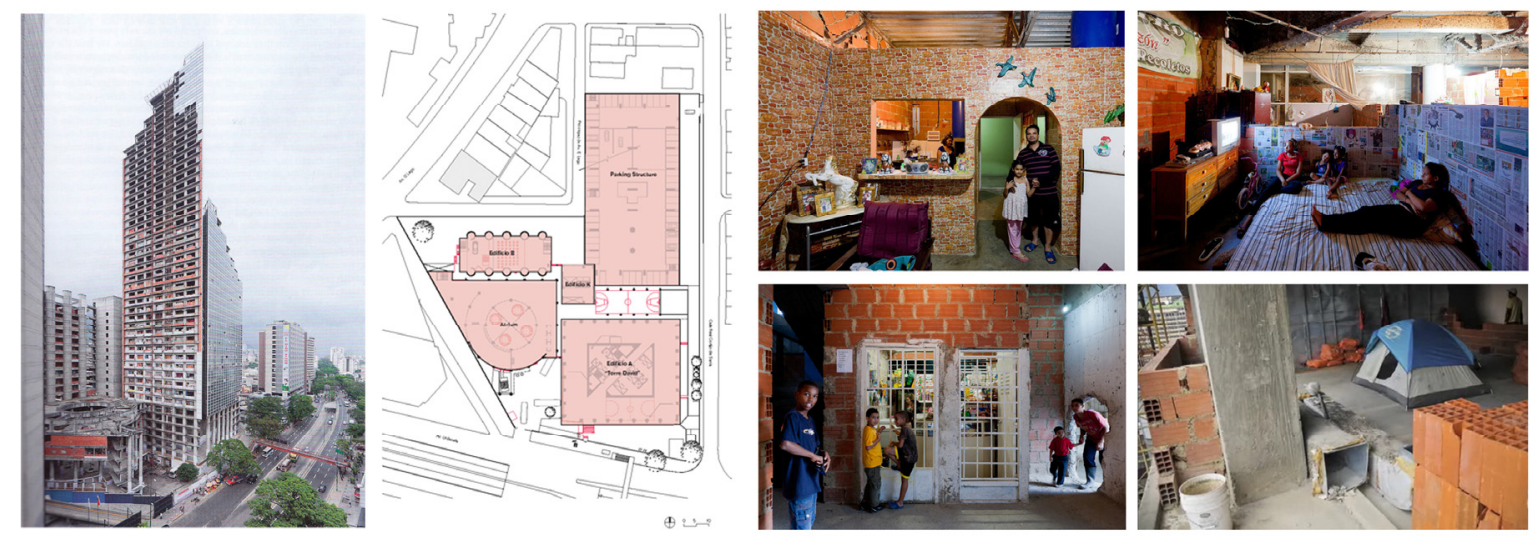

Figure 17 Images of Torre David (Brillembourg \& Klumpner, 2012)(Google)

Another argument is that providing flexibility and involving homebuyers fails to provide the lucrative economy that developers and investors are expecting (Till \& Schneider, 2005). This is also untrue. Japan already shows that flexible building tends to last longer and is 
cheaper in the long run without need for wholesale refurbishment. In addition, a Taiwan architect, Hsieh Ying-Chun, famous for his bottom-up construction and building processes with locals, did a detailed calculation for an urban design making use of open building techniques (Figure 18). He proved that such a design would provide more useable space for public facilities or housing expansion and subsequently provide more economic benefits. His design 'City for people' was submitted for a housing design competition held in Shenzhen, China, and won first prize. His design was a fixed urban structure that would allow residents to make flexible housing decisions. He also stated that a prior condition for design techniques of this nature is that the land must be public-owned (ATD, 2012). This demonstrates the benefits of such a design ideology.

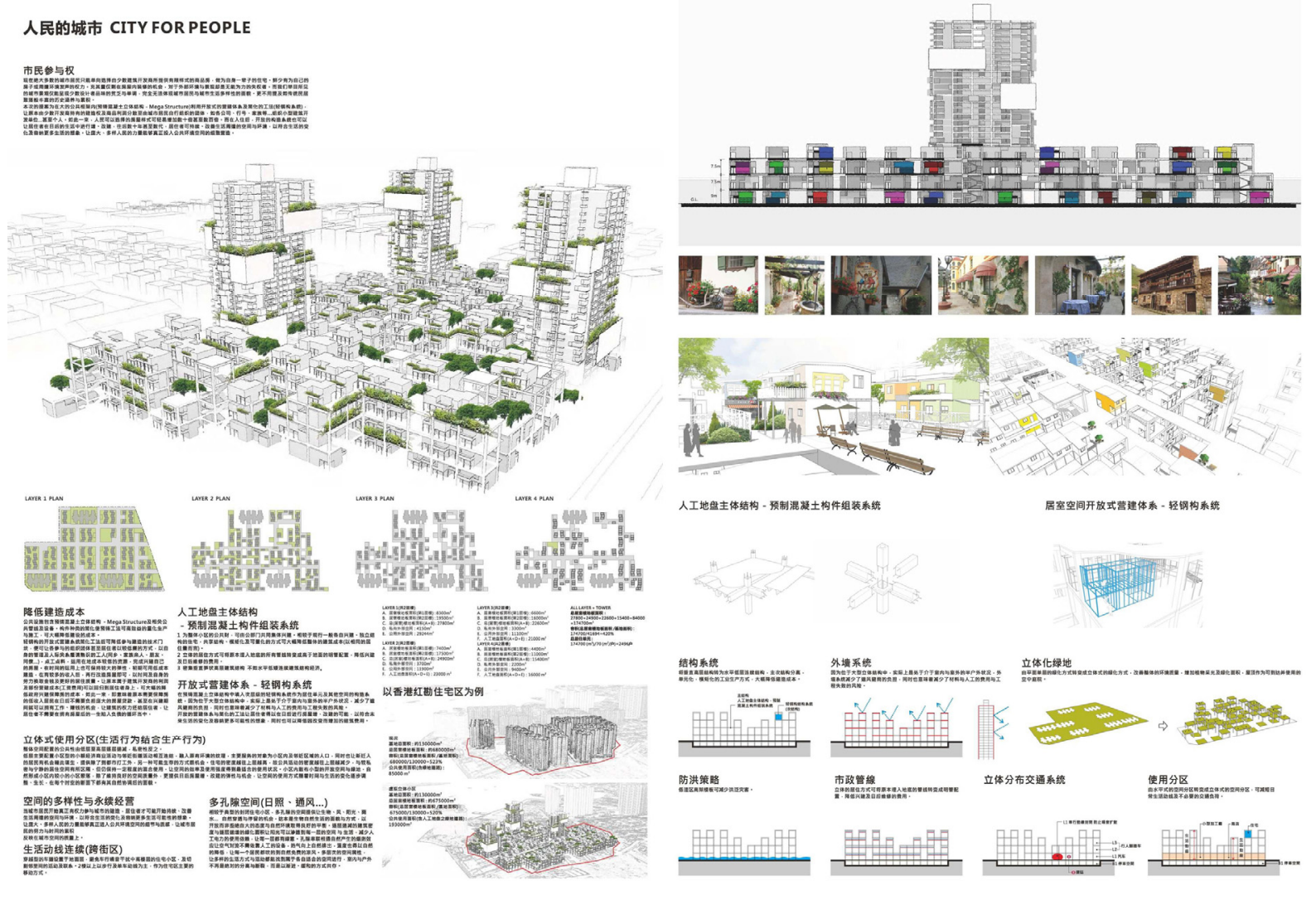

Figure 18 Winning design for housing competition by Hsieh Ying-Chun (ATD, 2012)

The shifting trend can also be seen in other initiatives which could provide indirect support to the open building design movement. One of these is Open Source Architecture (OSA). In product development, open source is a philosophy that promotes universal access 
via free license to a product's design or blueprint, and universal redistribution, including subsequent improvements to it by anyone (Smuts et al., 2010). Opening source code enables diversified customization to further develop original products past the limitations of the initial version. This is the basis of collective intelligence for innovation (Gao et al., 2015). OSA is an emerging paradigm that advocates new procedures in imagination and formation of virtual and real spaces within a universal infrastructure (Smuts et al., 2010). In the architectural field, the ideas and the approaches of open source design have been borrowed for years. However, it has not yet been applied to mainstream architectural practice because of the complexity of the architectural industry. This includes, but is not limited to, design, procurement, construction and many other intertwined issues. But again, the potential to apply it to OB should not be overlooked.

Another indication of shifting trends is the increasing application of a technique being incorporated into the OB concept: mass customization. Termed by Stanley Davis in his 1987 book "Future Perfect", this process was formally systematised by Joseph Pine in 1993. Pine defined mass customization as the production of individually customised goods and services. It relates to the ability to provide customised products or services through flexible processes, in high volumes and at reasonably low costs (Pine, 1993). The process of mass customization is a multi-faceted one and includes many various aspects, from managerial to technical. This production strategy aims to provide customers with individualised products with near mass production efficiency (Blecker \& Friedrich, 2006). Kieran and Timberlake (2004) argue that mass customization has increasingly influenced construction processes and components over the past few decades. Most recent production approaches, which employ specific digital design environments and manufacturing processes, relate to the concept of mass customization, although its influence is at times discrete.

A major challenge of mass customization strategies in architecture lies in evaluating the efficacy of a product. It must be concurrently customizable, properly designed, in concordance with design codes and regulations, and accurately manufactured. Consumer 
products are usually modularized in a way that partially limits customization for technical pragmatism. Architecture, however, is unique in its interlinked structure of responsibilities. In the design, production and verification processes of creating a building, there is usually no single party with the necessary specialisation in all areas to manage the project. Accordingly, realising a mass customization design and fabrication environment requires a high level of communication between users, designers and manufacturers. Fragmentation poses a major obstacle, as fabricators in the building industry generally consist of small- to mid-size companies whose production volumes are insufficient for generating the economy-of-scale effects of modularized production in a typical mass customization model (Kieran \& Timbrelake, 2004). These aspects were derived from general theories and approaches to mass customization, and situate the user, designer, and manufacturer in a complementary relationship via direct or indirect communication. Buildings, however, are products whose design involves typological, cultural and social aspects that are yet to be thoroughly considered in the customization process. Despite this, the increase in demand for personalized goods and products that contributed to the call for mass customization is slowly influencing the building industry as well.

Confirming findings from the analysis of past examples, it might be true that providing flexibility and involving homebuyers means incurring more costs. In all previous cases, it can be observed that this 'cost' is not solely monetary; there is the need for more time and effort in managing, the need for the cooperation of government authorities and most importantly, the desire of the homebuyers must not be lacking. Reluctance is understandable from the developers' point of view. However, increased cost is a short-term drawback. The investment can be offset against long-term factors such as a higher sense of ownership of the units, encouraging homebuyers' involvement, reducing occupant fluctuation and improving the capability to react quickly to the changing needs and desires of existing and potential homebuyers, and also to those of the housing market (Till \& Schneider, 2005). This is much 
needed at present where there is a higher rate of uncertainty around future homebuyers and housing demand.

Rapoport (1983) compared building design with music. He contended that architectdesigned buildings are like jazz music, which is stylish, and the rest like popular art or folk music, belonging to 'mass culture'. He is suggesting that buildings designed by architects lack the spontaneous character of urbanism, which represents certain values about lifestyle. Rapoport also suggested that by observing what houses the people buy, a comparison between people's choices and architects' designs can provide insight into the needs, values and desires of the people. However, this research argues that without the participation of the people, the observation of choices remains constrained by what the architects provide. By allowing the bottom-up involvement of homebuyers, spontaneous results could provide more accurate feedback to better understand demand.

Kendall stated in his book (Kendall \& Teicher, 2000) that there is already a growing trend towards $\mathrm{OB}$. This is reflected in investment patterns, construction, manufacturing and, most importantly, is enabled through advances in information technology that areenhancing design and building processes. This could subsequently result in better design for housing flexibility and involving homebuyers. The recipient of 2016's Pritzker Architecture Prize was Alejandro Aravena, whose design projects all focus on the design participation of homebuyers. This further indicates the changing trend and recognition of these design processes among the community.

In the chapter that follows, this research looks at how technology has advanced to involve homebuyers in the development and decision-making of the housing design process. Based on the analysis of this chapter, various technological tools and the concepts behind them are examined to determine if they respond to the issues raised by homebuyerparticipation and contribute to a methodology that could enhance this process. Table 2 below provides a summary of the analysis and findings of the examples in this chapter. 
Chapter 2 | Mass housing from Anticipation to Participation

\begin{tabular}{|c|c|c|c|c|c|c|c|c|c|c|c|c|c|c|c|c|}
\hline 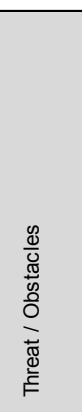 & 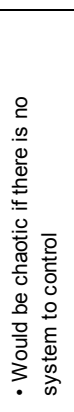 & 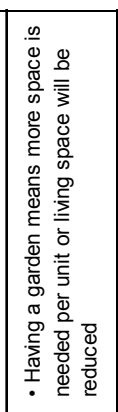 & 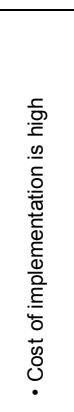 & 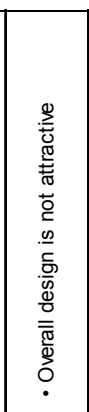 & 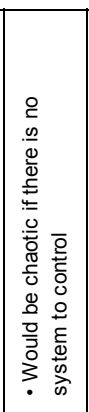 & 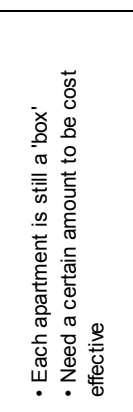 & 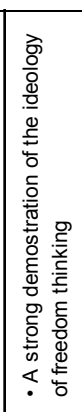 & 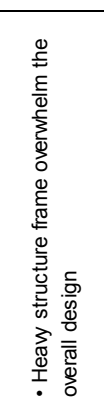 & 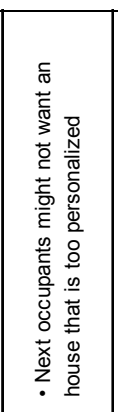 & 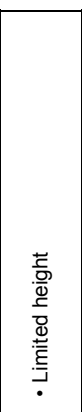 & 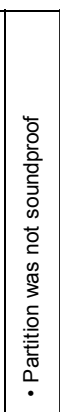 & 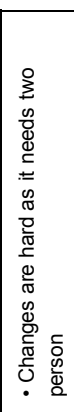 & 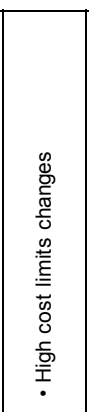 & 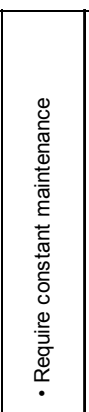 & 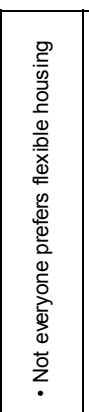 & 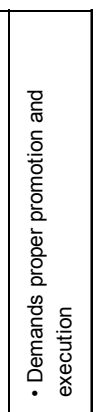 \\
\hline $\begin{array}{l}\text { 訔 } \\
\text { 言 } \\
\text { 응 }\end{array}$ & 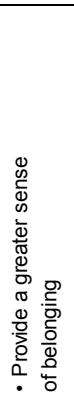 & 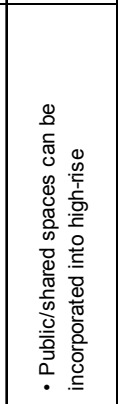 & 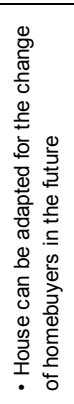 & 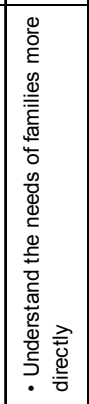 & 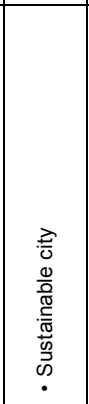 & 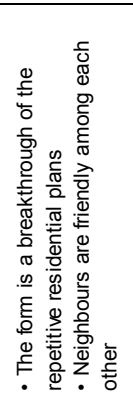 & 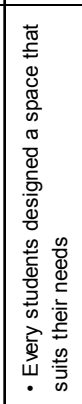 & 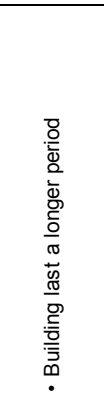 & 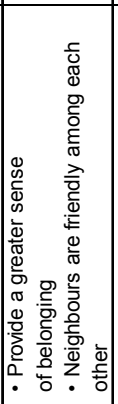 & 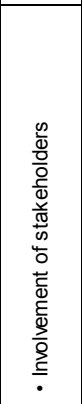 & 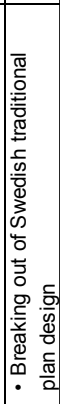 & 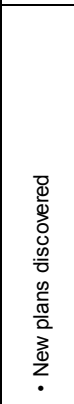 & 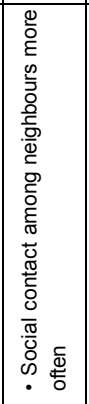 & 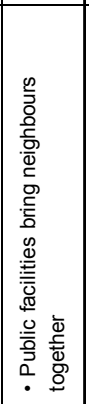 & 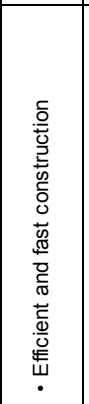 & 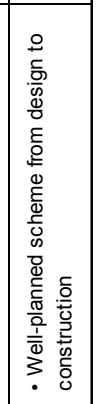 \\
\hline 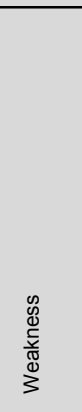 & 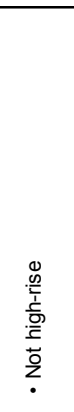 & 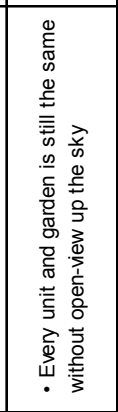 & 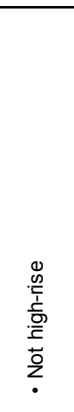 & 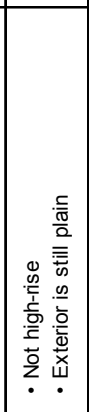 & 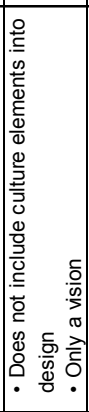 & 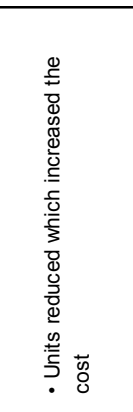 & 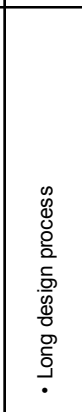 & 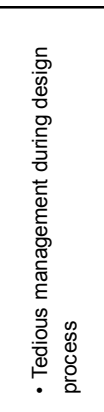 & 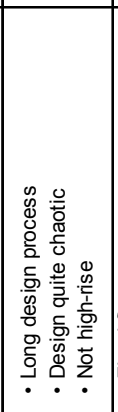 & 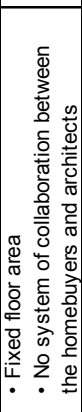 & 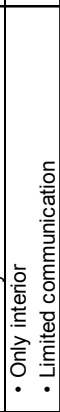 & 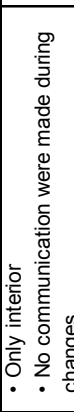 & 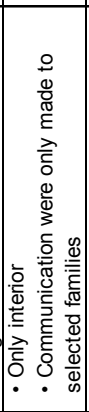 & 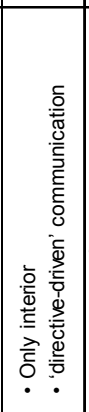 & 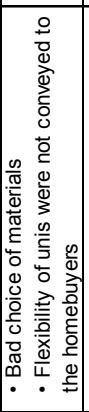 & 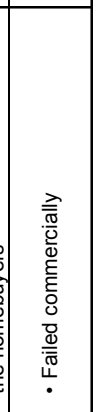 \\
\hline 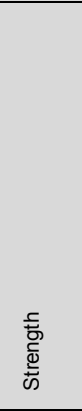 & 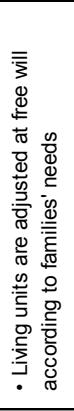 & 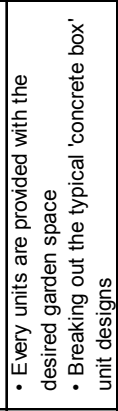 & 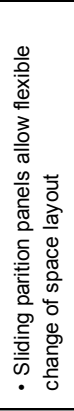 & 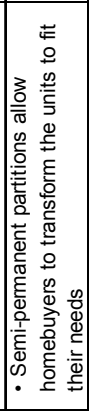 & 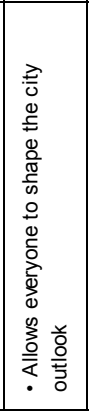 & 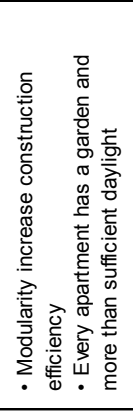 & 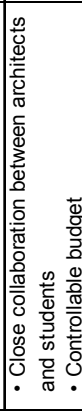 & 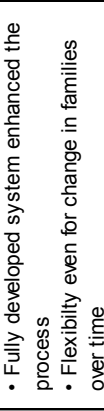 & 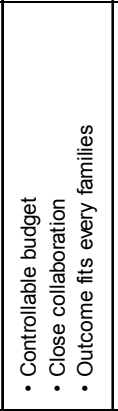 & 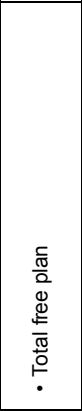 & 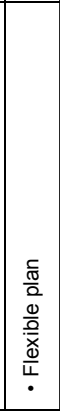 & 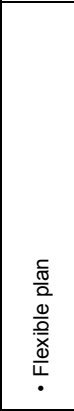 & 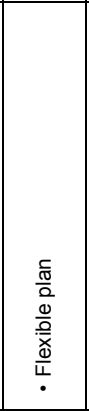 & 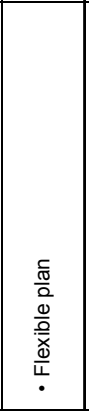 & $\begin{array}{l}\frac{c}{\frac{0}{0}} \\
\frac{0}{\frac{0}{3}} \\
\frac{1}{4} \\
\end{array}$ & 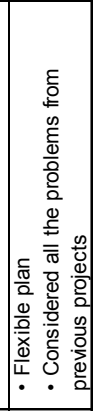 \\
\hline 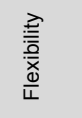 & $\stackrel{\infty}{\nu}$ & z & $\stackrel{\infty}{\succ}$ & $\stackrel{\infty}{\nu}$ & $\stackrel{\mathscr{D}}{\succ}$ & z̊ & $\stackrel{2}{2}$ & $\stackrel{\mathscr{D}}{>}$ & $\stackrel{8}{2}$ & $\stackrel{\mathscr{D}}{\succ}$ & $\stackrel{\infty}{\succ}$ & $\stackrel{\mathscr{D}}{\succ}$ & $\stackrel{\mathscr{D}}{\succ}$ & $\stackrel{\infty}{\nu}$ & $\stackrel{\mathscr{D}}{\nu}$ & $\stackrel{\circlearrowright}{\succ}$ \\
\hline 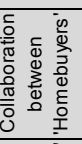 & $\stackrel{\mathscr{\infty}}{\rightleftharpoons}$ & $\stackrel{\circ}{z}$ & $\stackrel{\circ}{z}$ & 우 & $\stackrel{\circ}{z}$ & z & $\stackrel{\mathscr{g}}{\rightleftharpoons}$ & zo & $\stackrel{\infty}{\succ}$ & $\stackrel{\circ}{z}$ & $\stackrel{\circ}{z}$ & $\stackrel{̊}{z}$ & qo & 우 & zo & $z$ \\
\hline 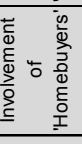 & $\stackrel{\infty}{\rightleftharpoons}$ & $\stackrel{2}{z}$ & $\stackrel{\infty}{\nu}$ & $\stackrel{\mathscr{D}}{\rightleftharpoons}$ & $\stackrel{\mathscr{D}}{\succ}$ & \&̊ & $\stackrel{\mathscr{D}}{\rightleftharpoons}$ & $\stackrel{\mathscr{D}}{\rightleftharpoons}$ & $\stackrel{\mathscr{D}}{\rightleftharpoons}$ & $\stackrel{\infty}{\rightleftharpoons}$ & $\stackrel{\infty}{\succ}$ & $\stackrel{\infty}{\succ}$ & $\stackrel{\mathscr{D}}{\supset}$ & $\stackrel{\infty}{\rightleftharpoons}$ & ż & 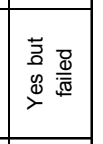 \\
\hline$\stackrel{\text { Ф }}{\succ}$ & $\stackrel{\circlearrowright}{\check{I}}$ & 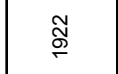 & $\underset{\sigma}{\stackrel{\Xi}{\sigma}}$ & $\widehat{\widehat{\sigma}}$ & 芯 & $\stackrel{\stackrel{\circ}{\circ}}{ }$ & ફे & $\stackrel{\text { 。 }}{\circ}$ & $\stackrel{\stackrel{\circ}{\circ}}{\circ}$ & స్ & \% & $\begin{array}{l}\stackrel{\leftrightarrow}{\stackrel{\circ}{\circ}} \\
\end{array}$ & 疋 & స్ & స్ & $\stackrel{\widehat{\sigma}}{\stackrel{2}{2}}$ \\
\hline 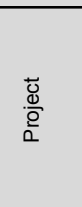 & 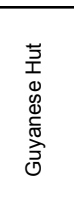 & 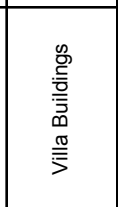 & 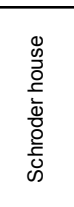 & 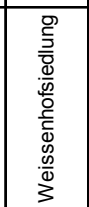 & 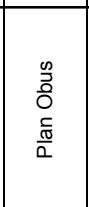 & 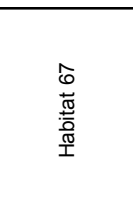 & 离) & $\frac{\vec{x}}{\frac{\tilde{x}}{2}}$ & 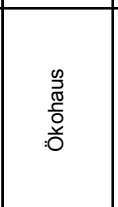 & 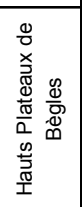 & 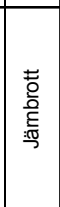 & $\begin{array}{l}\frac{\sqrt{0}}{0.0} \\
\frac{0}{3} \\
\frac{0}{3}\end{array}$ & 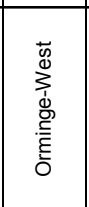 & 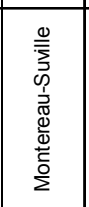 & 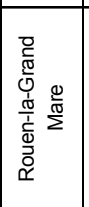 & 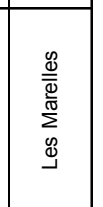 \\
\hline
\end{tabular}

Table 2. Summary of examples used in this chapter with its analysis 


\title{
Chapter 3: Towards a Technological Mass housing Synergy
}

\begin{abstract}
We must face the fact that we are on the brink of times when man may be able to magnify his intellectual and inventive capability, just as in the nineteenth century he used machines to magnify his physical capacity. Again, as then, our innocence is lost. And again, of course, the innocence, once lost, cannot be regained. The loss demands attention, not denial.
\end{abstract}

-Christopher Alexander-

(1964)

The summary table in the last chapter indicates that the process of incorporating homebuyers in the design process for mass housing faces many obstacles. There are multiple issues to resolve to ensure a transparent and practical process. The most common weaknesses of the projects are interconnected: The long design duration, and inefficient communication with homebuyers. Due to the lack of an efficient communication medium, the design process requires the architect to communicate with homebuyers individually, or with a team of architects as in the case of Next21. In most cases communication is through countless meetings, as in the case of Ökohaus and La Mémé. The demand for manual labour and the time-consuming communication adds a heavy burden to the cost of the project; a great threat to such a design process. There is a need to develop improved methods. In addition, mass housing is a collective design process but most of the projects do not have collaboration between homebuyers. The challenge is to develop a means of communication between architects and homebuyers, and between homebuyers, to achieve a higher level of collaboration for mass housing design.

Technology advances have seen the development of architectural design tools such as Rhino-Python ${ }^{\mathrm{TM}}$ script, Grasshopper, and Autodesk Revit ${ }^{\mathrm{TM}}$ that focus on Building Information Modelling (BIM) and could aid architects in providing alternative and better solutions for housing design. The development of 'Citizen Design Science' by Gerhard Schmitt (2015) and the 'Eco-building' toolkit on 'Kickstarter' by Marcin Jakubowski (2016) also allow 
layperson involvement in urban and housing design. In this chapter, this research studies various digital tools that allow the involvement of homebuyers with the design process. The aim is to analyse the capability of the tools in terms of allowing homebuyers' participation and providing design variations. The previous chapter found that it is possible for a layperson to design their own living space according to their needs. This chapter seeks to understand how each system empowers the homebuyers to design their living space, individually or collaboratively, without many difficulties. An important note is that the focus of this chapter is examining the level of homebuyers' involvement with various digital tools implemented for such a purpose, and the possibilities for enhancing the process. Although flexibility is part of the analysis in the previous chapter, it is a method used to allow the participation of homebuyers. This research uses it only to better examine the progress of such a design process. This research still makes use of such flexibility in housing design, and this is further explained in later chapters.

There are three main sections in this chapter. Firstly, the problems of participatory communication are discussed and how these could be addressed with a synergistic system. The complexity is also be explained, and the next section examines the various tools that have already been developed to provide for the complicated involvement of homebuyers with the housing design process. Lastly, the tools are compared with the examples of the previous chapter to outline a discussion of challenges and observe if the issues are addressed. This will set the stage for identifying the gap that is the focus of this research.

\subsection{Creating Synergy}

Although Alexander stated that participation would create chaos, he also believes that technological advancements will magnify humanity's intellectual and inventive capability (Alexander, 1975). The capability of technology and the widespread access to it has transformed communication, between people, and how they obtain new information. The idea of mass participation, once thought impossible, can now be achieved through the network. 


\subsubsection{BRINGING ABOUT DESIGN PARTICIPATION IN OTHER PRACTICES}

Product design, such as that of sports shoes by Nike, has used the internet (NIKEiD) to allow consumers to customize their own sports shoes (Wong D. , 2011). Governments can be seen using it (Paris, 2014) to get public opinions before implementing urban policies that do not reflect the needs and desires of the people. Software such as MetroQuest ${ }^{\mathrm{TM}}$ (MetroQuest, 2015 ) is also emerging to provide a platform for industry to engage with the public and obtain valuable information that could help spearhead development more efficiently. In science, researchers are using public engagement to solve problems that scientists have spent years on, and yet failed to find a solution for (Mohammadi, 2014). The potential of participatory design is endless, yet in the context of housing it still faces many challenges and doubts by the public authority. Habraken stated in an interview that (Lüthi \& Schwarz, 2013):

The architect wants to, but he can only do it if the industry is willing to deliver the built-in elements. The industry is ready to produce but wants to be sure that investors and dealers are willing to use these products. The people financing it would gladly do it, but they want to be sure that public authorities are willing to support such initiatives. And the public authorities, in turn, are wondering if architects are able to do it and if the users really want it. And so we are stuck in a vicious cycle, which can only be broken if enough people are convinced about the urgency of the problem.

Despite the many examples from around the world promoting the involvement of homebuyers in design, the advantages have yet to encourage authorities and developers to break out of this vicious cycle and adopt collaborative design processes more generally. This research aims to tackle this by putting the focus on homebuyers. If design participation can gain more support among the people (or 'users' as Habraken puts it), it could reverse the cycle and influence other stakeholders along the chain to incline towards this design direction too.

\subsubsection{SOLVENET}

It is challenging to provide a means for the public to participate in projects. The SMART Method of Public Policy founded by Oprea Radu Adrian stated that participative processes face the following challenges (Oprea \& Sarpe, 2009): 
- Unwillingness to dedicate time because this process does not provide any personal economic benefits.

- Organizing collaborative projects requires investment which might not have any direct returns.

- Discussions are sometimes monopolized by one or a few dominant members and requires experienced facilitators - again requiring time and money.

- A lack of knowledge may result in poor-quality output. This is undesirable and more prevalent in projects of short duration. Again, time is necessary to allow participants to understand the project and think about the issues to provide better solutions.

- Ineffective processes due to a lack of accountability. The participatory process demands transparency, but it is hard to obtain access to people's data to assess their input. The participants are not confident that their input will influence or impact the project and therefore do not dedicate themselves to the process.

The idea behind the SMART method is to use a digital tool, SolveNet, to provide a social platform that allows everyone to raise a problem of public concern, suggest solutions and push it to the extent of effecting policy. It utilizes the advantages of 'social networks' which Brian E. Adams states, in addition to 'time, money and civic skills' (Verba, Schlozman, \& Brady, 1995), are important for the participation of people. This creates opportunities for people to mobilize each other, fosters civic virtues, helps develop communication skills and raises awareness of public issues. (This is before Mark Zuckerberg brought his social network online in 2004.) By making the participatory process online, it addresses the problems of time and cost. Participants can use the platform at the time and location of their choice, while still able to connect to other participants. They can also use the internet to gain a better understanding of the issues and spread this knowledge quickly.

SolveNet endeavours to address the problems mentioned above, and Oprea also tries to 'move past the civic stereotypes and create environments that will appeal to real citizens' (Oprea \& Sarpe, 2009). His idea is based on Matt Leighninger's (2006) book 'The Next Form of Democracy' in which the participatory process focuses on citizens rather than the goals and agenda of the organizers. Opera also increases the appeal of such participation by giving 
recognition and credit to those who are helpful and constructive. This motivates participants to be responsible for their inputs and results in a more accountable process.

Although these strategies are for participation in the development of public policies, they provide useful observations and indicate why participatory processes in the housing context face the situation described by Habraken. Participation in housing design faces the same challenges: It is difficult to find homebuyers willing to dedicate time for such a tedious design process (Lendt, 2012). Concomitantly, authorities are focused on supplying housing units and do not feel the need to spend extra effort to involve homebuyers and potentially threaten the order in their system (Gao et al., 2015). Oprea's observation can serve as a guide in determining the factors that must be considered for this research.

In 2007, the UK government agreed to create a bill on climate change based on the government sponsored 'Stern Report' by Nicolas Stern (Osborne, 2006). The main reason for the government to finally act after numerous appeals on the negative effects of climate change, is that as an economist Stern explained the problems in a 'language' which made sense to politicians (Wood, 2007, p. 2). This implies that simply making the homebuyers the focus might be insufficient. Providing fast and effective means of engaging homebuyers in an economically sound way, could better attract various stakeholders to willingly support the process.

\subsubsection{JOHN WOOD}

The first thing that comes to mind is to develop a digital tool to facilitate the participatory process. However, compared to government policies and urban developments, housing appears to be a much more personal matter; for many, the chance to own a house is like a dream come true. But in big and dense cities, such a 'dream' is limited to only homogenous apartments. It requires more than a communication tool for every homebuyer to engage in design collaboratively and break out of monotonous design in a bottom-up manner. This, to a 
certain extent, coincides with John Wood's definition of 'micro-utopia'. He stated that the idea of utopias by Sir Thomas More '... not only courts criticism of being 'unrealistic' or 'idealistic' but also has a whiff of revolutionary fervour' (Wood, 2007, p. 2). But he also stated that it is necessary to 'dream', especially in today's pragmatic culture which has reduced long-term vision, and promote visionary, optimistic projects which can bring about real changes. $\mathrm{He}$ therefore introduced the idea of micro-utopia, 'a more tentative, temporary, pluralised or truncated version' of utopia and, most importantly, achievable (Wood, 2007, p. 2). This research takes a similar ambitious stand with John Wood's ideology to develop a system that could enhance the collaborative participation of homebuyers to bring about a better version of housing design processes and outcomes.

Looking deeper into Wood's design for micro-utopia, he uses the contexts of politics and economics to question the current situation and examine how a different system might be developed to enable everyone to participate and contribute to the system, achieving a global 'synergy of synergies' (Fuller, 1975). He suggested some ways in which everyone's microutopias can be brought together, simplified into five steps:

- Step 1 - breaking the first barrier that is neither technological nor political but psychological: Willingness and daring to dream beyond what is attainable.

- Step 2 - bringing people together to co-imagine the dream in a more shareable form. This involves exchanging dreams and seeing how each can enhance another.

- $\quad$ Step 3 - checking that the dream suits what they need.

- Step 4 - determining how many of those dreams are attainable.

- Step 5 - working together to share the tasks of producing and sharing the dream.

This is a very useful reference for developing a system of participatory housing design. The objective is to use design participation as a starting point for achieving new possibilities, and hopefully to subsequently bring about harmony and, indirectly, political and economic development. 


\subsubsection{THE SYNERGY}

Wood's interpretation of synergy is what truly inspires this research to adopt the ideology of micro-utopia. In common terms, a 'synergistic system' is understood as situations or conditions in which the result exceeds the sum of its parts and where 'synergy' simply means collaborative. In other words, connecting each other's micro-utopias collaboratively is more beneficial than just putting them together. Therefore, the principle of synergy is that it encompasses more than the exchange between individuals and the system and includes that between individual parts and their separate relationships to the system (Wood, 2007, p. 149). This means that the systems are complex and, no matter how carefully the process is planned, the outcome unpredictable and emergent (Fuller, 1975), potentially diverging from the initial design aim and intention. To better manage this 'intelligent form of sharing' (Wood, 2007, p. 148), a design practice that differs from that of design done for 'performance' and 'efficacy' is necessary. Wood offers four provisional orders of synergy to better identify the different clusters of synergy types (Wood, 2007, p. 151):

- First Order of Synergy: synergies that emerge from the sharing of data (facts and figures).

- Second Order of Synergy: synergies that emerge from the sharing of information (processed data that are meaningful and useful).

- Third Order of Synergy: synergies that emerge from the sharing of knowledge (information and skills acquired over time and experiences).

- Fourth Order of Synergy: synergies that emerge from the sharing of wisdom (synthesis of knowledge that provides deep insights).

To better grasp the aspects of a complex system, Wood implies it is necessary to work with clear data sets and map out synergistic relationships using a nodal diagram (Figure 19). 


\begin{tabular}{|c|c|c|c|}
\hline Nodal Diagram & Feature & Comprehensiveness & Implications \\
\hline & $\begin{array}{l}2 \text { players } \\
1 \text { relationship }\end{array}$ & Very high & Minimum collective potential \\
\hline & $\begin{array}{c}3 \text { players } \\
3 \text { direct relationships }\end{array}$ & High & $\begin{array}{l}\text { With more players, relationships starts to } \\
\text { become indirect }\end{array}$ \\
\hline & $\begin{array}{c}4 \text { players } \\
4 \text { direct relationships } \\
2 \text { intersect relationships }\end{array}$ & Comprehensive enough & $\begin{array}{l}\text { Team misunderstanding may build up if } \\
\text { collaborators have to also deal with } \\
\text { intersecting relationships which is confusing }\end{array}$ \\
\hline & $\begin{array}{c}5 \text { players } \\
5 \text { direct relationships } \\
5 \text { intersect relationships }\end{array}$ & $\begin{array}{c}\text { Exceeds the scale of } \\
\text { exclusively direct relationships }\end{array}$ & $\begin{array}{l}\text { Going beyond the average person's conscious } \\
\text { grasp }\end{array}$ \\
\hline & $\begin{array}{c}6 \text { players } \\
6 \text { direct relationships } \\
9 \text { intersect relationships }\end{array}$ & $\begin{array}{c}\text { Very rich but increasing hard } \\
\text { to manage and more } \\
\text { dependent on intuitive } \\
\text { management }\end{array}$ & $\begin{array}{l}\text { Increasingly relies heavily on experiences, } \\
\text { and/or intuitive skills and insights }\end{array}$ \\
\hline
\end{tabular}

Addition possible configuration with only direct relationships
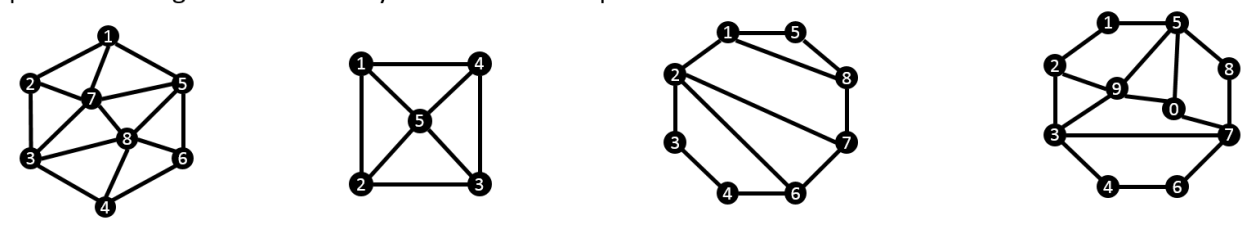

Figure 19 Nodal diagram showing the number of players and the implications to comprehend the relationships (Wood, 2007)

With the increase in the number of players, the number of relative relationships increases exponentially. Any attempt to deal with each relationship between all players consciously becomes practically impossible. To provide a more practical example to explain the nodes and relationships, the case study of 'BedZED' is mapped accordingly (Figure 20). The Beddington Zero Energy Development (BedZED) is a successful example of innovative, zero-energy, sustainable housing on a multi-unit scale (Andrews, 2008). Its success was not its reliance on new, sophisticated building technologies, but its ability to gain support from groups of interested parties, each with their own different interest. It's fascinating to consider why this type of project did not happen earlier, and why there is no equivalent successful scheme after (Wood, 2007, p. 164). By breaking down the complex system into parts, it is easier to identify the sustained synergies required for future projects to adopt. 


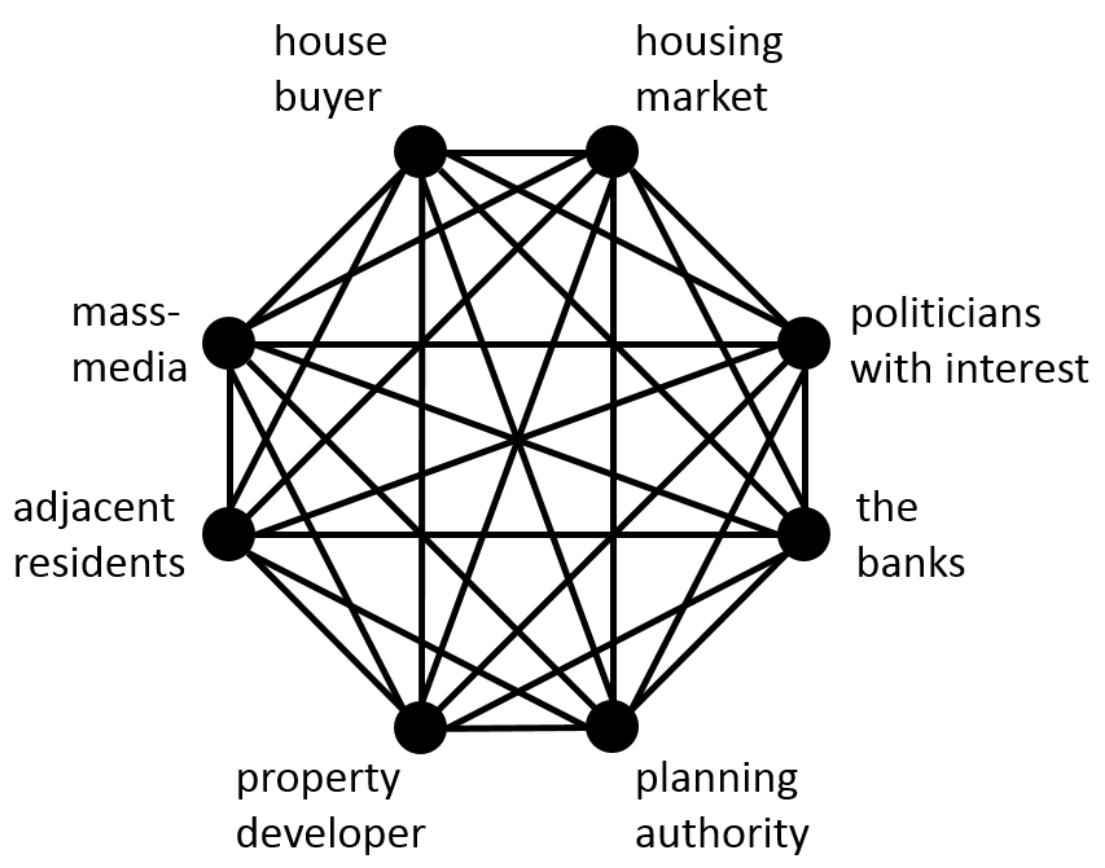

Figure 20 The players and relationships of BedZED (Wood, 2007)

With only 8 'players' there are already 28 possible relationships within a project. This can be further magnified to some 56 relational viewpoints. The map provides a clear picture of the example, and presents an opportunity to devise a new solution for a situation very similar to the housing context in this research. The challenge is therefore to not only develop a digital tool for the collaboration of homebuyers, but also to devise a framework that could deal with specific relationships accordingly and bring about the kind of synergies that Wood describes. The next section looks at some digital tools that have been developed by researchers and practitioners to incorporate homebuyers into the design process.

\subsection{Needs for Technology Capability}

Lucien Kroll, in his book 'The Architecture of Complexity' (1987), suggests 'computer use in design' (CUD) instead of 'computer aided design' (CAD). He claimed that the former is more representative of his vision of future tools, allowing more open-endedness and the integration of architectural; design and social relationships. He criticized CAD for being like artificial 
intelligence, leading to designs that are more 'self-contained, closed and repetitive' (Pak \& Verbeke, 2014). He employed various algorithms to generate diversity and differentiation for his users, but was limited by the technology of his time. He emphasised that computers simply generate, evaluate, and modify designs which still require revision by architects, and that social interaction must not be lost in order to deal with the diversity of the real world. He wrote a full chapter outlining the need for technology capable of handling complex processes and he still believes it is necessary for the present social situation (Kroll, 2013).

Enabling homebuyers to design their own house is nothing new, there are already several tools developed with some being commercialised. However, the extent to which these systems provide for a synergistic approach is what this section hopes find out.

\subsubsection{WIKIHOUSE}

When it comes to flexible housing and using digital tools to involve homebuyers, the first system to come to mind is the WikiHouse. It was a project initiated by Alastair Parvin and Nick Lerodiaconou to promote an online, open source construction kit that allows people to design and build themselves a new house - even without professional construction skills (Labarre, 2011). The process is designed to be simple: Homebuyers go to the WikiHouse website and select their desired house layout and design. The system then automatically generates jigsaw puzzle-like pieces from plywood which are then sent to a CNC (Computer Numerical Control) milling machine to cut out the parts. The pieces can then be easily transported and constructed (Figure 21) with instruction manual attached. The first official WikiHouse was built at the Gwangju Design Biennale in South Korea in 2011 and several WikiHouses have been built since in other countries (Mens \& Vreeswikj, 2016).

The innovation of WikiHouse is not in using new technology, but in bringing the technology into the public domain (Parvin, 2012). Integrating parametric software tools (specifically using Sketchup ${ }^{\mathrm{TM}}$ plug-in) into WikiHouse lowered the threshold to design and build a house. The idea to encourage self-build housing can be dated back to the 70 s by Walter 
Segal who designed a self-build housing system based on timber frame construction (Spatial Agency, 2011). Parvin has enhanced this process with the help of technology.
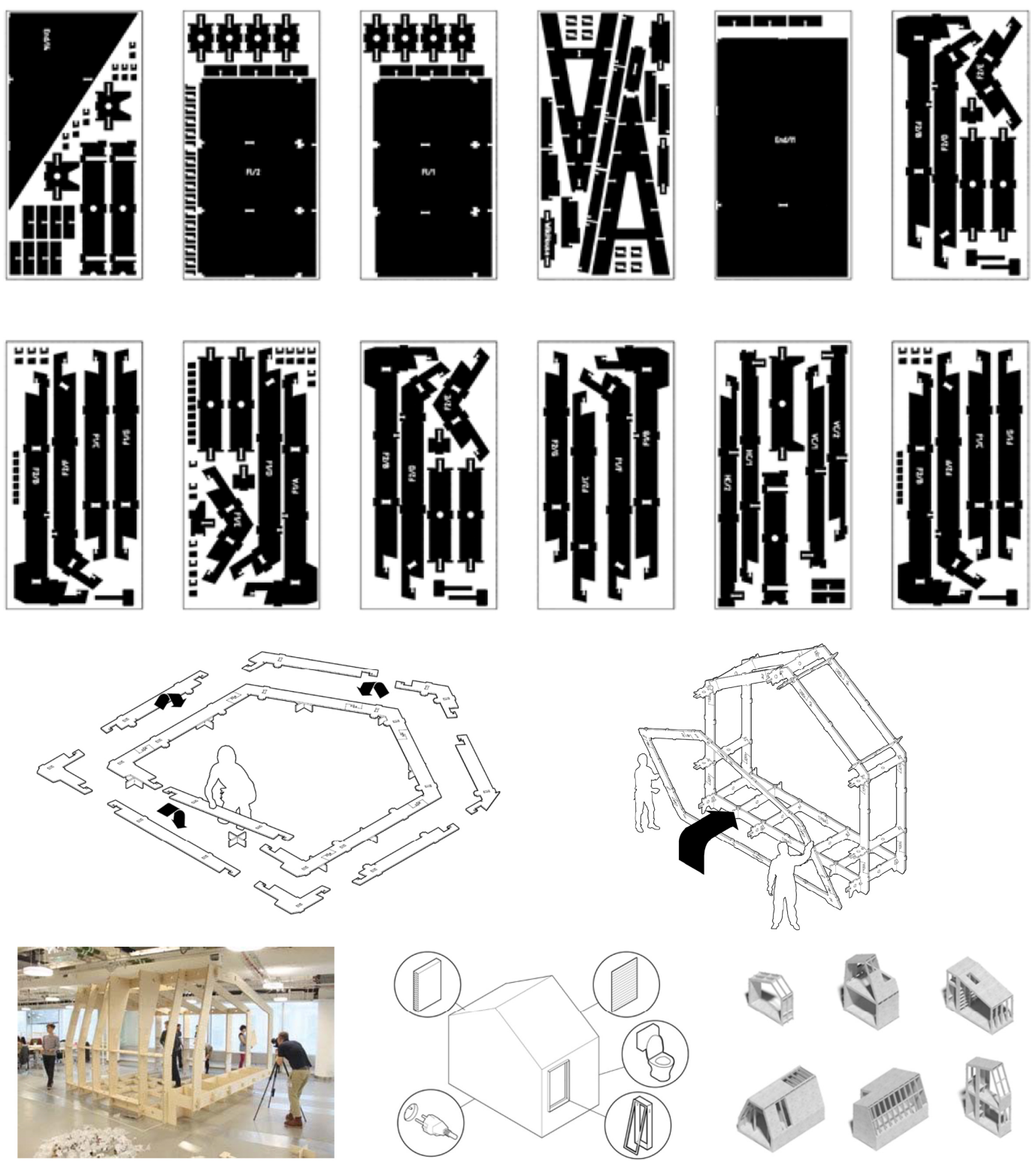

Figure 21 Assembly of WikiHouse and varied outcomes (Labarre, 2011) (Parvin, 2013)

He pushed it further and made everything open-source by publishing all information under Creative Commons, a website that allows anybody to share anything to everyone without monetary gain. His aim is to make design and knowledge accessible to everyone (Parvin, 2012). This posts a great challenge as it means the project depends greatly on funders, supporters and collaborators. 
Looking into WikiHouse gives this research an initial impression of how digital systems can be used to engage the public and extend to the construction process. Comparing it to Wood's synergy model, WikiHouse did provide the technological means to achieve the five steps towards everyone's micro-utopia, but to a limited extent. WikiHouse is a system for individual houses. Participation in design is only between individual families and the system. Interaction with architects happens only when requested, or if architects are interested enough to be involved in the process. In other words, the cooperative part is not fully realised. The sharing aspect of WikiHouse can be considered to reach only second order as useful information like plans and construction details are open-sourced.

This is not, however, the complete picture of WikiHouse. The EDFAB research group at the University of Auckland used the idea from WikiHouse and digitally fabricated a sleepout (McMeel, 2015). The group extended the research further to include BIM capabilities and improve the qualities and performances of the potential house. As Parvin states, what they have done so far is just a small experimental part of a big picture, in which only the structural assembly is looked at. Many other aspects, from housing systems such as ventilation and sanitation to democratizing the housing market, can be included to fulfil the potential of WikiHouse (Parvin, 2012). That is to say; there is a possibility that WikiHouse could realise fully Wood's micro-utopia and bring about a high level of synergy among the communities.

\subsubsection{WIDE}

What WikiHouse cannot provide is potentially understood through other systems such as 'WIDE', a Web Interactive Design Environment developed for homebuyers in Taiwan to customize their housing interior layout to suit their needs (Chien \& Shih, 2000). Land cost in Taiwan is high so apartment units are sold to homebuyers before being built, to allow developers the capital to realise the projects. The system is designed to allow homebuyers to customise their purchased units before construction begins. With all the customizations within 
a project, a large amount of information must be documented and exchanged among agencies. WIDE is, therefore, a system that could be used to support three main functions: Customization processes; management and information exchanges with a focus on human-computer interaction; information management (Chien \& Shih, 2000).

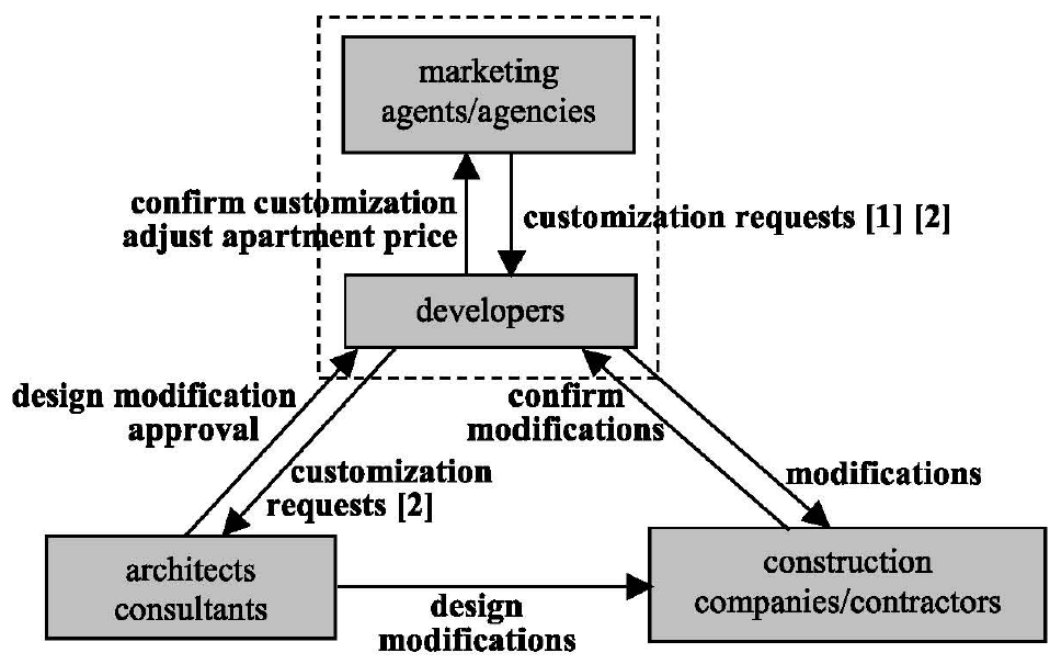

Figure 22 Customization process in Taiwan housing (Chien \& Shih, 2000)

There are four key users with developers being the central figure (Figure 22). Homebuyers' customization usually goes through marketing agents and developers. If the changes become too complex, they go through architects for approval. This customization process also reflects the hierarchy of design and how design is communicated in the Taiwanese context. With WIDE, the first focus, design interaction, is arranged into three levels (Chien \& Shih, 2000):

- Interior finishes/equipment selection: Homebuyers can make changes to finishes and furniture which are reflected through computer rendering and a virtual reality walkthrough experience.

- Layout arrangement selection: Homebuyers can choose from a library of pre-designed floor plans to suit their needs.

- Advanced layout adjustment: Homebuyers can further customise the interior layout through system design aids. 
For information management, the system architecture (Figure 23) of WIDE was designed to facilitate an exchange between stakeholders. It includes the calculation of cost for customizations, provides the necessary feedback and stores data in a central database eliminating conflicting file versions among stakeholders.

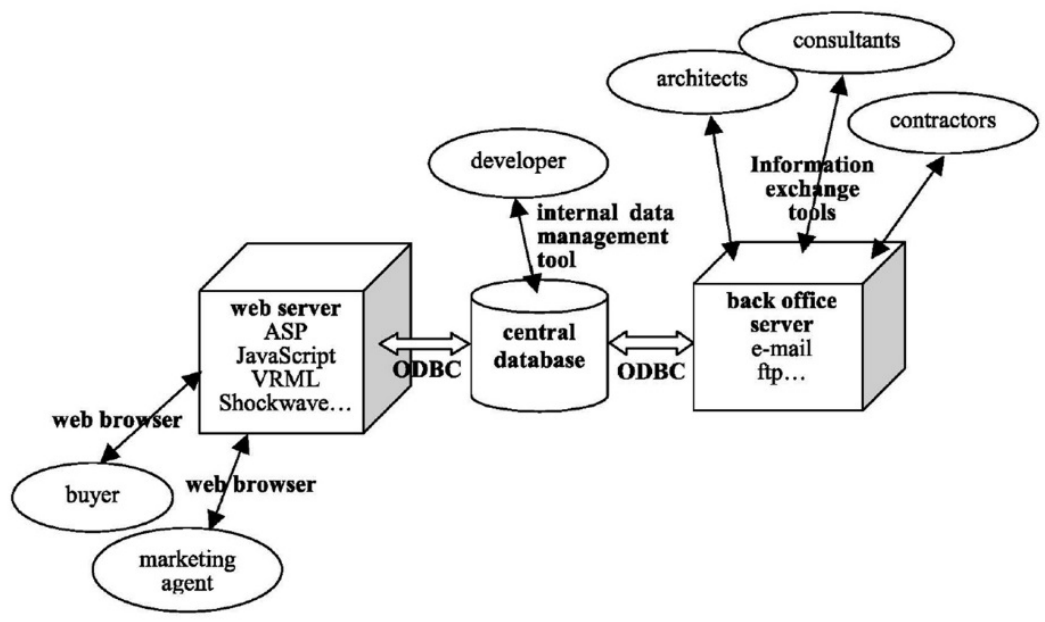

Figure 23 System Architecture of WIDE (Chien \& Shih, 2000)

Kindom Construction Corporation, a developer in Taiwan, used this framework to develop WIDE-Kindom (Chien \& Shih, 2001). It is one of the earliest prototypes using web browsers to examine using the internet to provide real-time modifications and visualization of buildings with the direct participation of homebuyers. As the system is geared towards developers, there is no back-end service provided to the architects, consultants and contractors shown in Figure 23. However, WIDE-Kindom does have a central database using 'Microsoft Access' and a more intensified front-end web-server that provides five services for the participation of homebuyers: virtual open house; community information; apartment selection guide; buyer customization centre; Feng-Shui recommendations. Homebuyers can customize the apartment units with the help of the apartment selection guide to fit their preferences and needs. The interface (Figure 24) provides a left panel for components selection and instant view of changes made, and a right panel to show the recommended options and cost estimation. 

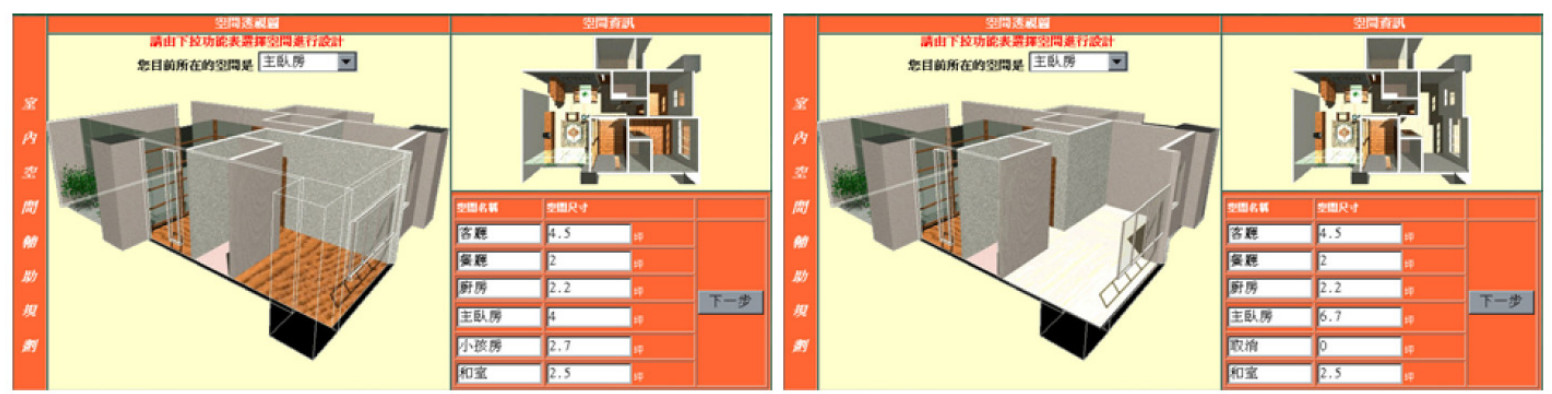

Figure 24 Interface screenshot of WIDE-Kindom (Chien \& Shih, 2000)

WIDE-Kindom provides a rich set of interactions for homebuyers to select the apartment units of their choice. As this system is for apartment building projects, it provides great insights for this research when determining how such techniques can also be applied in other contexts. Although WIDE-Kindom provides well-rounded information for homebuyers, it is still highly limited in terms of design collaboration and communication.

The involvement of homebuyers is at the level of pre-designed plan selection. The technology provides a database for more variations of plans and more information for homebuyers' reference. The only design element in the system is for homebuyers to select partitions and remove them to merge spaces and change finishes around the interiors. So, if one homebuyer prefers to have the living room smaller for a bigger bedroom, unless there is a pre-designed plan for it, the homebuyer cannot achieve it.

As for communication, without a back-server, WIDE-Kindom is only for the developers and the homebuyers. There is no clear and direct communication between the two within the system. The homebuyers interact with the system individually, and the data obtained by the developers for the next phase of the project.

What WIDE-Kindom provides is similar to WikiHouse; they have tried to include the homebuyers with the design process but not to the extent which Wood is trying to achieve in his micro-utopia. Apartment building projects, where groups of homebuyers interact within one project, are a great opportunity to try and establish Wood's model within the system. However, without communication between homebuyers and with only information being put 
across, the level of synergy which WIDE-Kindom provides can only be at the second level. Third level synergy can only be achieved when the homebuyers are using the system, and developers or architects are also present to provide knowledge and guidance. This is also the case for WikiHouse when there is direct communication between the architects and the homebuyers during the construction of the house. The difference between WIDE-Kindom and WikiHouse is the stage of the design process on which they focus.

The constraints of WIDE-Kindom are due to the limitations of the technology of the time. As the number of design options increases, more web script layers are required, and to include the user interaction, the web browser could not handle the additional layers. However, the system did manage to fulfil its aim of supporting the participation of homebuyers and information management. Moreover, a second prototype, WIDE-Roadhouse, was later developed. (Figure 25).
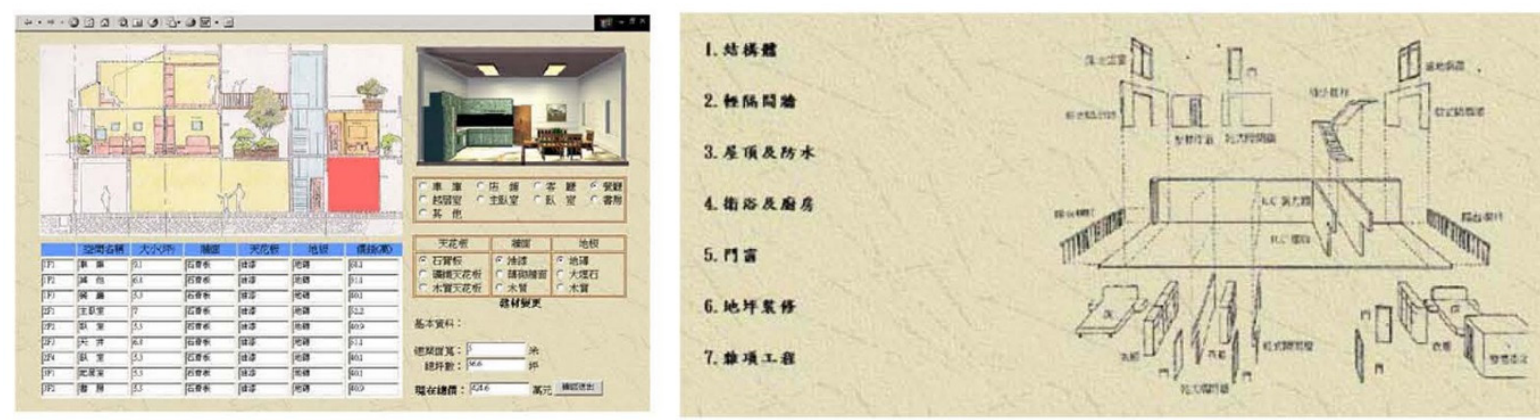

Figure 25 Interface screenshot of WIDE-Roadhouse and components of the building system (Chien \& Shih, 2001)

The second prototype breaks down architectural components so homebuyers can assemble them based on their needs. The level of customization was also increased to allow configurations of orientation, lot size, and primary functions. This provides a much more flexible design system for homebuyers to achieve what they need. However, as this prototype was created for another context (Chien \& Shih, 2001), the system is for houses and not for apartment buildings. 
The two prototypes of WIDE provide great insights into how the system can be structured to enable a more interactive environment for homebuyers, while maintaining the management needed for data collection. As most homebuyers do not have a design background, WIDE also separates complex design knowledge, or the lower level component (the search space), into a black box; while showing the system (the interface) as a glass box (Chien \& Shih, 2001). This is inspired by Karlgren (1994), and the idea plays an important role in the design of the participatory framework of this research.

\subsubsection{I_PREFAB}

With two systems explored, there are still many gaps to be filled. One of them is the connection between the needs of homebuyers and the designs. WIDE and WikiHouse provide numerous choices for the homebuyers, but these might not be sufficient. It is necessary to modify the process by understanding homebuyers' choices first. 'i_Prefab', a system that develops a choice model based on homebuyers' needs, could provide a missing piece of the puzzle for this research. This system has been developed by Joseph Huang from Illinois Institute of Technology for his PhD thesis (Huang \& Krawczyk, 2006).

In the United States, only five percent of people hire and pay an architect to design and build a home based on their needs (Huang \& Krawczyk, 2006). i_Prefab is developed to break out from the existing home design models (Figure 26) and use technology to provide a better means of communicating design with homebuyers and further reduce the extensive time required for design and construction. This could attract more adoption of technology of this type. 
Traditional Home Design:

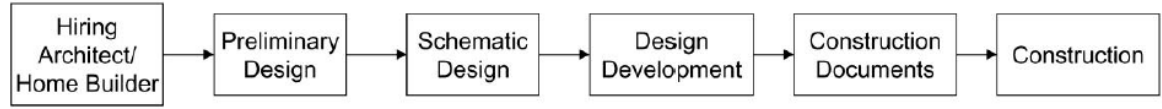

Factory-made Home Design:
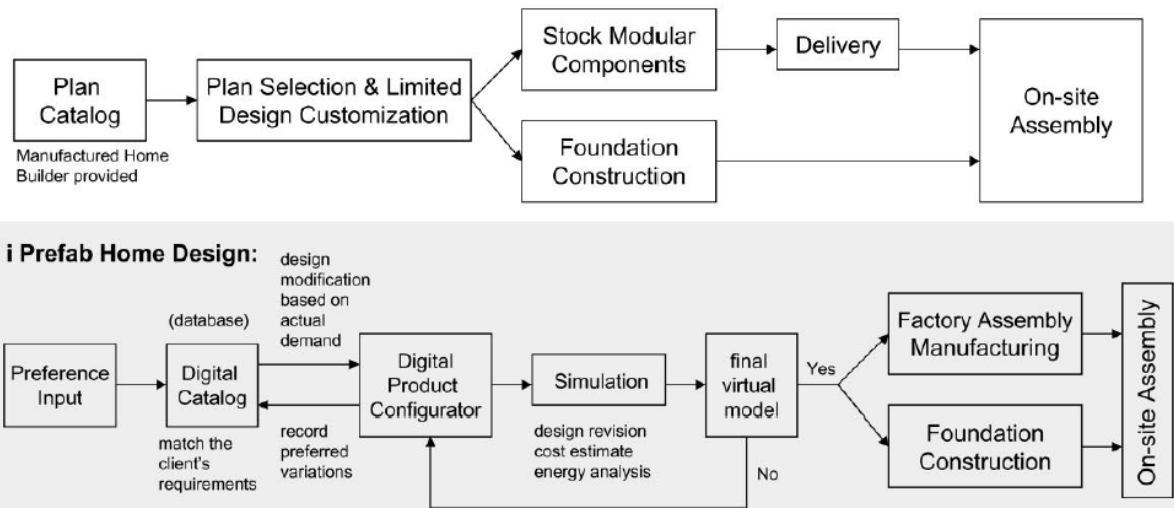

Figure 26 Existing models of home design and model of i_Prefab (Huang C.-h. , 2007)

Prefabrication techniques have been adopted in the United States for some time but have not flourished as expected as industry does not cater to the diversified needs of homebuyers. Still, companies such as Alchemy Architects, Sandonato \& Wehmann and Lazor Office have created customizable houses like weeHouse, kitHAUS and FlatPak respectively, based on different modular systems (Huang \& Krawczyk, 2006). Joseph found that these three housing systems are insufficiently customizable and flexible to fit a random test context of his choice. Moreover, those systems are still the typical 'catalogue-providing-homebuyers-select' process.

To facilitate the collaboration design process into a more homebuyers-oriented design interface, Joseph developed a series of multiple-choice questionnaires linked to respective pre-established answers, which can be translated into the architectural implementation of the homebuyers' needs (Huang C.-h. , 2007). The system provides real-time feedback; as the homebuyers answer questions, a display shows the outcome of their choices. This choice model utilizes TheBrain (a web interface by TheBrain Technologies) to map out a decision tree that can generate a maximum number of options with the least number of choices for homebuyers to make. To make the process clearer, the questions in the system are grouped 
into four levels; each a stage of the design process, gathering information specific to that stage (Figure 27):

- Level 1 - Generate list of required space (homebuyers' needs)

$>$ Info A - Household profile

$>$ Info B - Lifestyle

$>$ Info C - Activities at home

- Level 2 - Determine plan layout and room sizes (building geometry and space adjacency)

$>$ Info A - General site context

$>$ Info B - Lifestyle (spatial preferences)

$>$ Info C - Room size

- Level 3 - Define room layout and design

- Level 4 - Customise finishes and appliances 


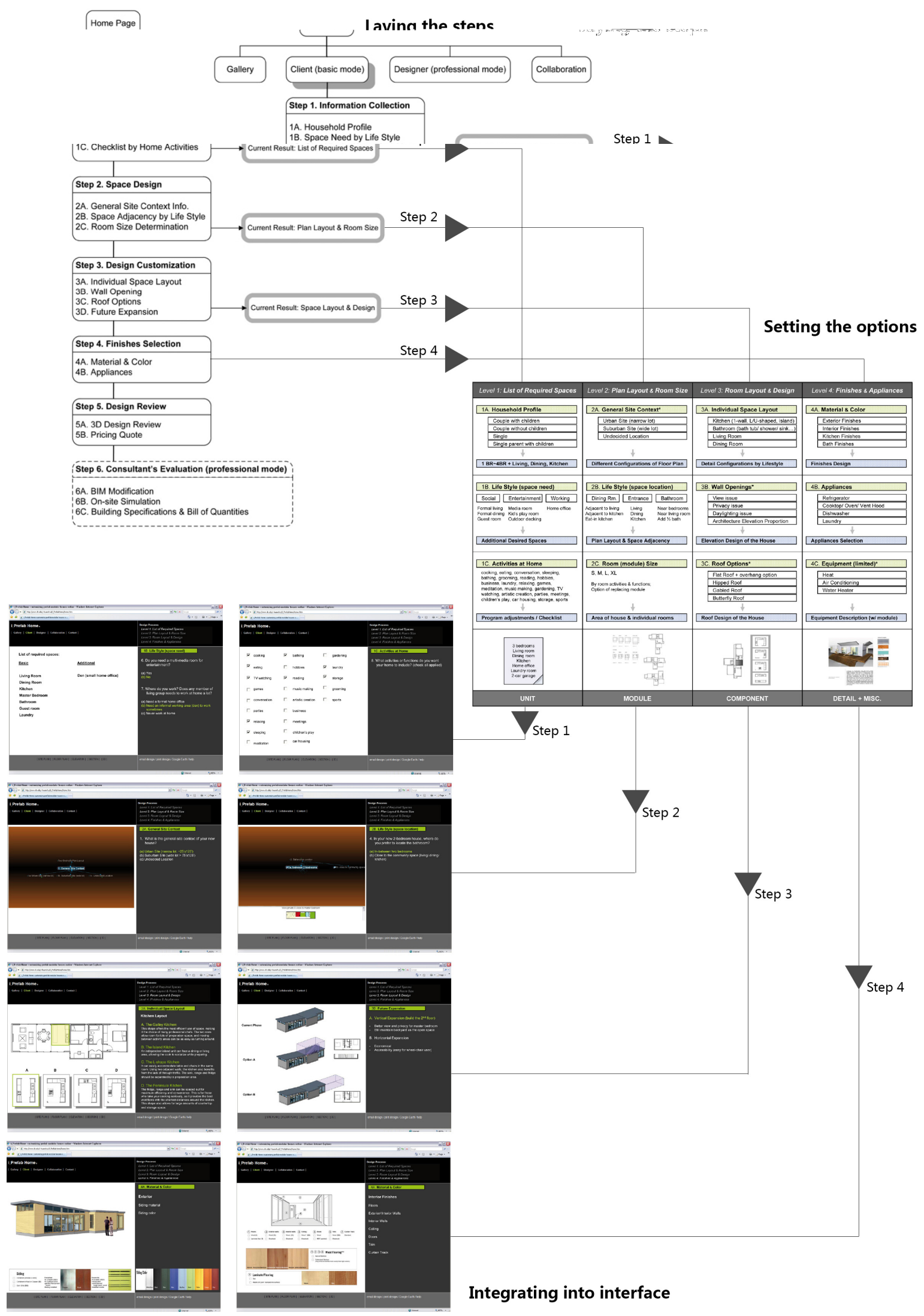

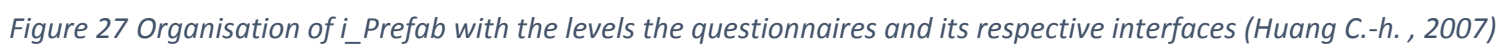


The first level seeks to understand the spaces needed by the homebuyers, even to the extent of knowing possible future needs, like family expansion, so that the design can cater to changing needs. The second level focuses on getting site information to make sure the design can fit the site boundary. This level plays a key role in generating layout by asking which space homebuyers want to see first when entering the house. This acts as an anchor point from which to start the plan layout. The third level of questions determine various room layouts, such as the kitchen and the connection between bedrooms and toilets. The last level is the selection of room finishes, and the house is designed. The questionnaire is well established as each decision made by the homebuyers provides the information necessary to generate an outcome. Moreover, at the end of each level, there is a summary of the information collected for homebuyers to confirm. Although the primary purpose of i_Prefab is to connect homebuyers with design and architects, it is also necessary that the generated design can be constructed. Therefore, BIM is integrated into the process so that the digital outcome can be exported into BIM applications such as Autodesk Revit for professional review and price quoting. The model can also be imported into Google Earth to ensure the design fits the site.

i_Prefab provides a comprehensive model of a method of obtaining homebuyers' input for spatial requirements, for organising them with an advisory tool, and for generating outcomes for architects to review and revise. However, there are still some downsides to this system. Firstly, it is only for individual housing. Secondly, homebuyers cannot manipulate design directly though the design options provided; customized alternatives can be quite extensive. Thirdly, homebuyers cannot visualise and experience the design outcome even with a simple walkthrough. Lastly and most importantly, although this system is for architects, salespeople and homebuyers, the only direct communication in i_Prefab is through an online help centre to provide assistance with the 
system. There is no direct design communication between the parties to establish a higher level of synergies than those of the previous two examples. Nevertheless, i_Prefab still provides valuable knowledge for this research to bring it to a residential apartment context.

From i_Prefab, it can be observed that prefabrication is no longer a series of repetitive production of product designs based on a predetermined set of standards. This is better replaced with 'mass customization'. Kieran and Timberlake stated in their book (2004, p. 111) that:

Mass production was all about the economy of making things in quantity, but mass customization does not depend on quantity to be cost effective. Mass customization is about cultural production as opposed to the industrial output of mass production.

\subsubsection{BARCODE HOUSING SYSTEM}

In other words, mass customisation, if formulated properly, could provide a cost-effective manufacturing solution while maintaining the capability to facilitate a variety of products. Barcode Housing System (BCHS) is a system which examines the use of mass customization in residential housing design to develop a digital environment for homebuyers. BCHS is developed by Madrazo (2009) for the participation of not just the users, but also other stakeholders such as manufacturers and builders (Figure 28). 


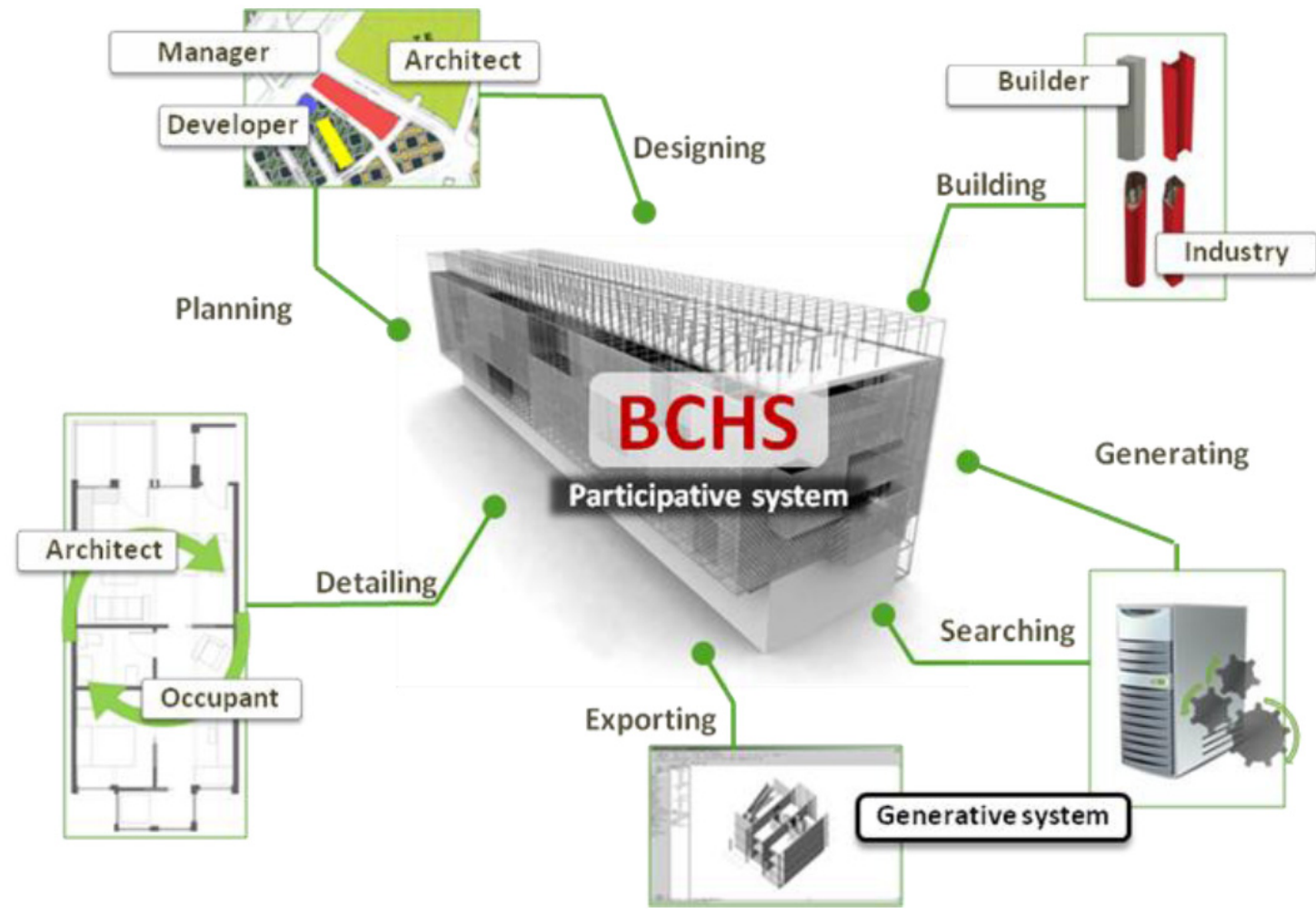

Figure 28 Collaboration of various stakeholders working concurrently within the system, allowing inputs from different users at any stage of the process (Madrazo, Rivera, Costa, \& Sicilia, 2010)

Compared to the previous systems, the housing layouts of $\mathrm{BCHS}$ are different; they are generated based on a parametric grid with each cell representing a type of space (kitchen, bedroom, toilet) and graphical values of its attributes (privacy, size), its edges indicating possible connections with other cells and specifying the degree of visibility and accessibility (no opening wall, door, window) (Figure 29). However, the detailed plans are still created from a pool of predesigned solutions in a database; though they are not as restrictive with each cell, in the shape of a bar, capable of varying its neighbouring cell based on the homebuyers' needs. One very important factor of BCHS is its application of the Open Building concept. By using the support/infill method of separating the building components and integrating the parametric grid technique into the infill element, the design becomes an open, flexible design that can be introduced for homebuyers' participation. 


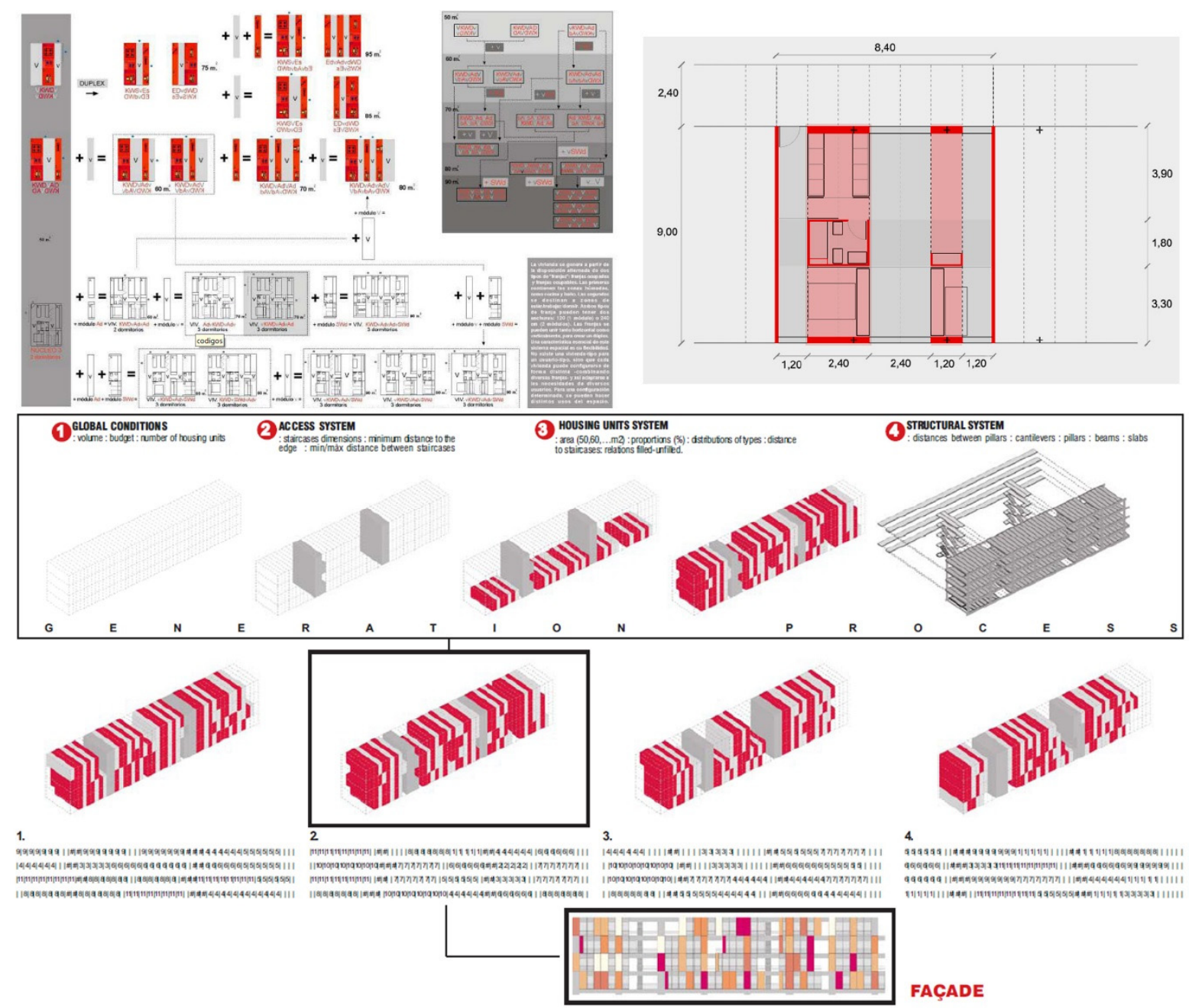

Figure 29 BCHS generating floor plans by merging parametric 'bars' of various functions and the use of open building concepts (ARC Engineering and Architecture La Salle, 2014)

The system architecture of BCHS is made up of six connected working spaces. Various stakeholders are involved in the design process in a synchronous and asynchronous manner at various stages of the whole building process, from design to construction as follows (Madrazo, Rivera, Costa, \& Sicilia, 2010):

- Project Development - this working space is for developers, architects and building managers to set the properties of the building projects such as site boundary, building height and volume and types of units.

- Housing Unit Layouts - the architects determine the set of cells that will be used for the generation of floor plans.

- Housing Unit Configuration -homebuyers are mainly in this working space to determine their housing design. 
- Housing Units Assembly - the architects define the parameters for assembling the housing units; the degree of compactness, for example, and the position of utilities.

- Housing units' specification - collaboration between architects and homebuyers happens here to set specifications for items such as finishes, partitions and furniture.

- Building Information Model - the information generated by the various stakeholders is collected and compiled into a comprehensive building description, to be exported for further review and in preparation for construction.

Although described as working spaces, these are also the different stages of a building project. Madrazo does so because he does not want a linear design process, but one where every stage can be happening concurrently, and where at any stage, should the design be found to be flawed or insufficient, the process can be rewound to make the necessary adjustments.

To engage with homebuyers more efficiently and effectively, the interfaces for the Housing Unit Configuration workspace are designed to allow homebuyers to use it easily. BCHS also utilizes questionnaires to simplify the engagement process. However, instead of using merely texts like i_Prefab, BCHS makes use of adequate terms, icons, images, colours and highlighting techniques and structures the sequences to only four steps; each with an interface of its own (Madrazo, Rivera, Costa, \& Sicilia, 2010). For this, the process is linear as preceding information provides input for the next step:

- Step 1 - Occupants and spaces (top-left of Figure 30): homebuyers input their family size, the room types they need and their respective sizes by selecting the choices available and clicking on icons in the interface. Additional information might include the kind of activities homebuyers think might happen in the various spaces.

- Step 2 - Spatial qualities (top-right of Figure 30): the homebuyers then build on their previously established activities to choose the kind of spatial qualities they prefer by choosing images with various qualities.

- Step 3 - Room interrelation (bottom-left of Figure 30): after the spatial qualities, the following step sets the connection between spaces. An initial version is generated based on the images selected at previous steps, and the homebuyers interact with the circles to further set the connections to suit their needs. 
- Step 4 - Plan layout (bottom-right of Figure 30): with the information collected from the previous steps, the system generates ten floor plans that fit the homebuyers' descriptions of their needs and preferences. Homebuyers choose the one they believe best suits their requirements and determine the accessibility between each space. The plans are shown as simple coloured blocks for easy interactions.
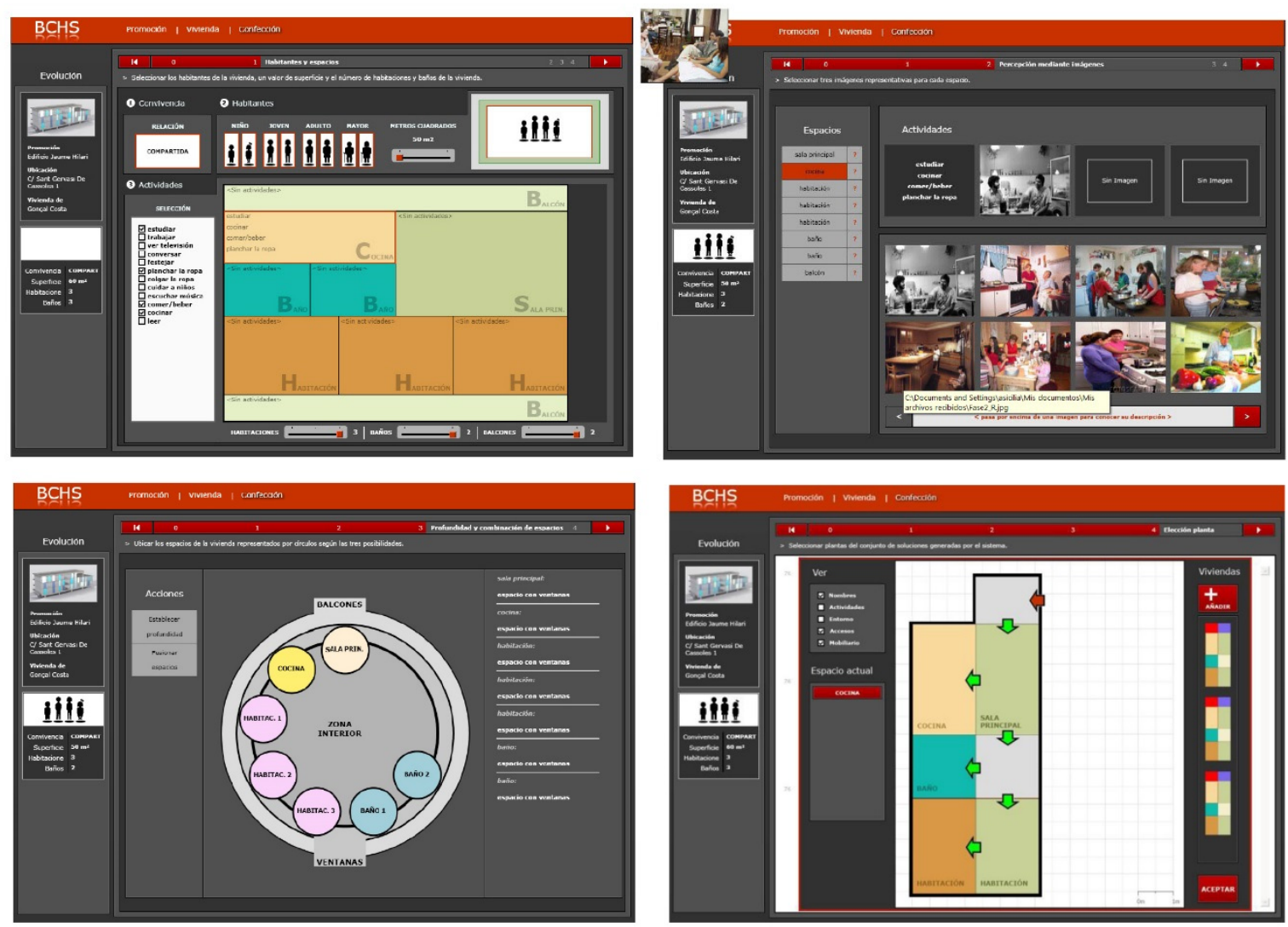

Figure 30 Screenshot of the four interfaces in Housing Unit Configuration of BCHS (ARC Engineering and Architecture La Salle, 2014)

The outcome is then collated by architects for assembly before being brought to the last interface, where homebuyers work with architects to work out interior space finishes and building components. This ensures that openings such as doors and windows reflect the final arrangements of the housing units. The interaction with homebuyers provided by BCHS is very similar to i_Prefab. The process is straightforward. It takes information from homebuyers and generates a design step by step from spatial configurations to spatial layout and, finally, interior finishes. The utilization of more visual-based interfaces for the interaction of homebuyers makes BCHS a more visually pleasing experience. However, it lacks the instant feedback 
where homebuyers can immediately see how their selection will affect the outcome. It is only after all information is collected that plans are generated.

The system architecture of BCHS also encompasses the generation of plan layouts (Madrazo, Sicilia, Mar, \& Angel Martin, 2009), information exchange and communication between stakeholders (Madrazo, Cojo, Sicilia, \& Costa, 2010) and using clustering techniques to select and merge cells into housing units from a repository (Sicilia, Madrazo, \& González, 2011). However, the focus here is on understanding how the systems are used to engage the homebuyers. As a result, these aspects are not described in detail but are referenced in a later chapter discussing the development of a prototype for the purpose of this research.

BCHS provides a comprehensive understanding of how a digital system, with the help of a parameterized design method and open building concept, could enable the design participation of homebuyers within an apartment housing context. The design methodology of this system is very close to what this research is seeking. However, the design process still interacts with individual homebuyers separately, without any communication between homebuyers. Even communication with the architects is established only at the end, during the choosing of finishes. Although BCHS tries to educate homebuyers throughout the design process (Madrazo, Rivera, Costa, \& Sicilia, 2010), there is a limit to what the system can achieve. Communication with architects and between homebuyers plays an important role in achieving the kind of synergy this research aims for.

Many other systems have been developed to enhance the participation of homebuyers with housing and apartment design. These include examples such as House-ID v.2 (Elmasry \& Farid, 2007), Hinterland Köln (Frohn, Rojas Toledo, Ong, \& Behrbohm, 2010), Toll Brothers (Toll Brothers, n.d.) and Creatomus (Puusepp, Loke, \& Kivi, 2017). Most of these systems are very similar to the examples presented in this chapter so far, and most focus on individual 
housing. By studying the systems, the various concepts and system architecture necessary for the design participation of homebuyers can be understood and applied to this research.

Alexander acknowledged the capability of technology, but he also claimed that such technologies cost people their innocence and required attention (Alexander, 1975). Even though his remark was in the 70 s, when technology had just started to thrive, it is still relevant today. The web provides easy access to all kinds of information; to the extent that anyone with the internet can learn about anything through the screen. To put it into context, housing design can be picked up by anybody. Even if a 'layperson' does not have any architectural background, it does not mean they are 'innocent' or have zero knowledge about designing a house. The participation process should not be limited to just collecting information from homebuyers within the boundaries of predesigned plans, but include collaboration with homebuyers to generate new possibilities. This research believes that the role of the architects is, therefore, not simply to design houses, but also to facilitate design communication and embrace the capability of homebuyers to generate an outcome both visionary and practical; suiting the needs of the homebuyers. The system should subsequently serve as a tool to facilitate such a collaboration process. The next section examines the gap between the available systems and the aim of this research and how a new method, gamification, can play an important role in achieving that aim.

\subsection{The Gap}

Table 3 shows the summary of the systems discussed in the earlier section. Comparing all the systems, a significant similarity can be identified; the systems only serve as an information bridge between the homebuyers and the architects. 
Chapter 3 | Towards a Technological Mass housing Synergy

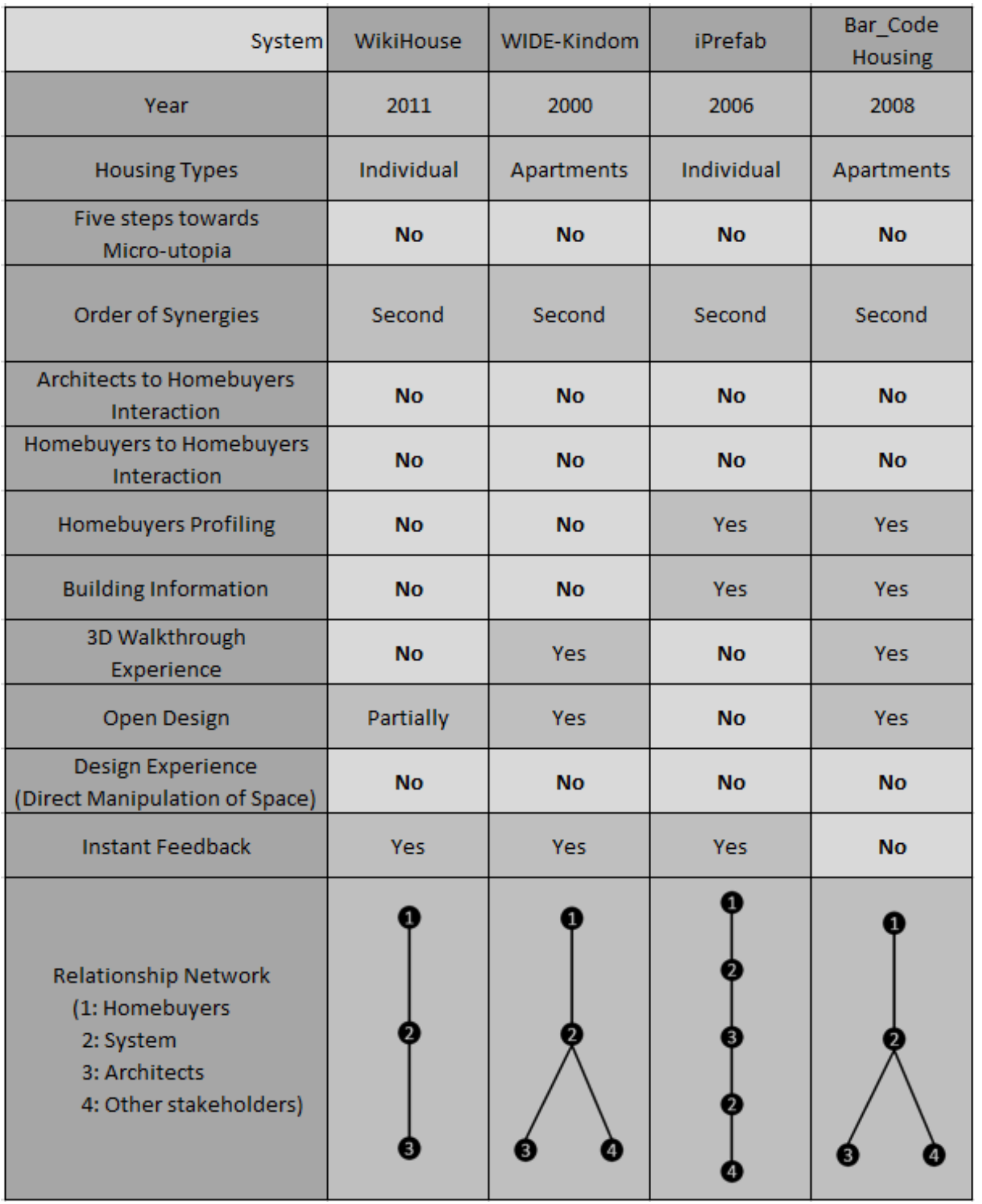

Table 3. Summary of system described in the previous section 
The architects pre-set architectural components and homebuyers interact with the systems to input their selections. The systems then use the information collected to generate a design and the architects use these to realise the project. This can be summarized as follows:

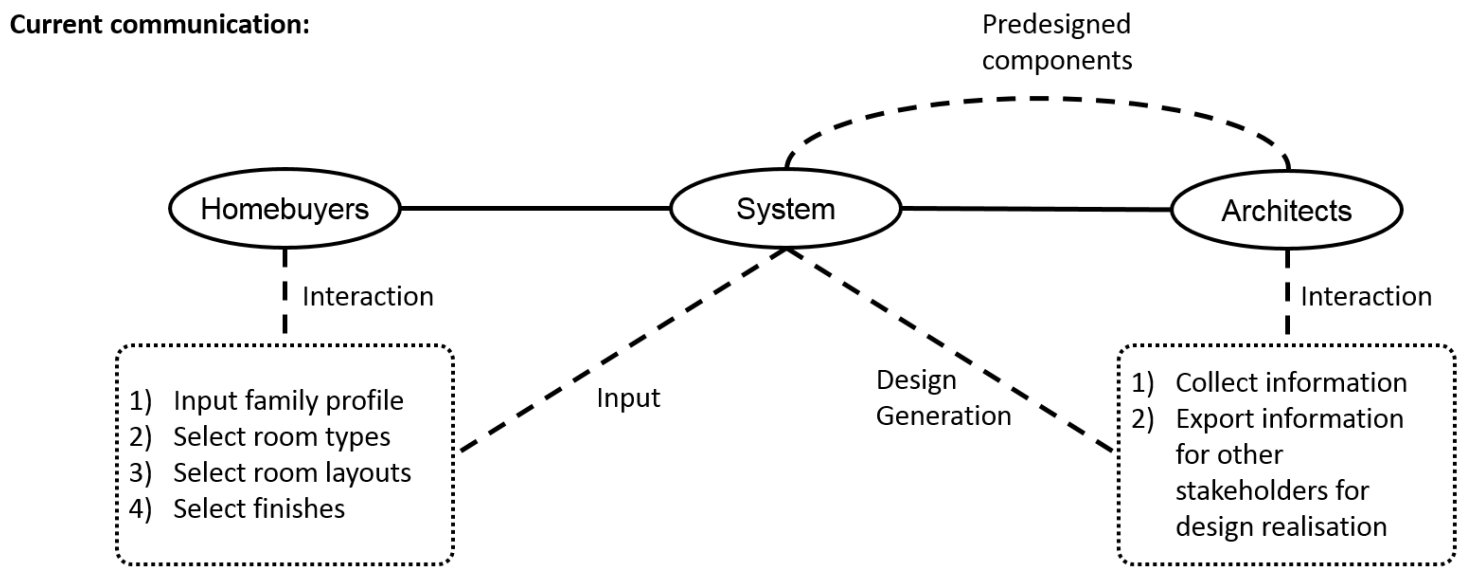

Figure 31 Current communication approach between architects and homebuyers with the system established

Reiterating the aim of this research, it is to identify the factors that could enhance the co-design participation of homebuyers in a residential context using a computational tool within a virtual environment. Through understanding of Wood's model of micro-utopia and the concept of synergy, this aim can be furthered to achieve at least the third level of synergy where knowledge is shared among homebuyers and architects. There should be a constant dialogue and exchange among them. The system then plays the role of facilitating synergistic communication to achieve design outcomes like those of Ökohaus and La Mémé, where the designs exceed the architects' expectations. The ideal relationship can be summarized as follows: 
Proposed communication:

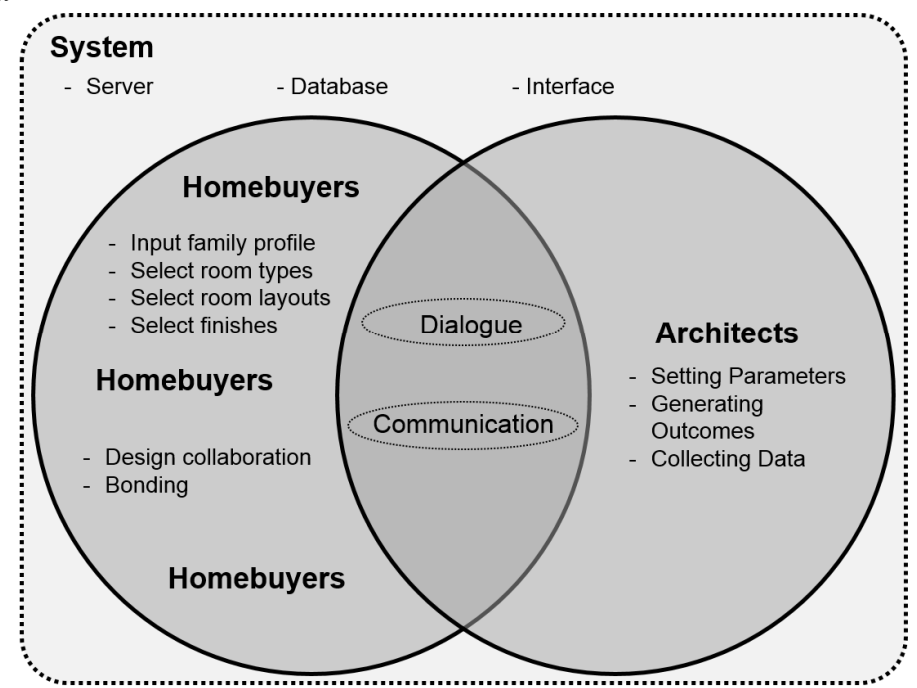

Figure 32 Proposed approach to achieve constant dialogue and exchange among the homebuyers and the architects within the system

Specifically, the gap identified through the study of existing systems is a need for a system that enables smooth communication between architects and homebuyers, and between homebuyers. More importantly, the available systems do not allow homebuyers to directly manipulate spatial design and architectural components. This research aims to enhance design collaboration and answer the research question using these factors as a basis.

\subsubsection{GROUP FORMING}

Before beginning this research, the involvement of the author with a master's thesis project by Ong (2013) provided a foundation for the establishment of the objectives for this research. Fabian's project is to develop a web-based design participatory tool for collaboration among homebuyers to create spatial and communal qualities of Group Form Architecture (Maki, 1965). Group Form Architecture is found in old medieval cities and traditional vernacular buildings. They emerged from a long period of spatial negotiation between neighbours while land was being urbanized and old buildings being replaced with new ones (Ong, Janssen, \& Lo, 2013). The desirable qualities of Group Form are in the spatial relationship, and architects like Koji 
Tsutsui have attempted to recreate these qualities using certain spatial rules. In his project, Annular Orphanage, in Uganda, Africa, he clustered rectangular housing units at 45 degree angles from each unit to the adjacent units (Tsutsui, 2010) to create pockets of space for communal activities. However, as the design is done in a top-down manner, it is missing the key elements for the homebuyers (in this case, the children as occupants) that would emerge if the design develops in a bottom-up manner.

The tool is developed in a residential context to focus on the design involvement of homebuyers with architects in a structured process consisting of the following three steps (Ong, Janssen, \& Lo, 2013):

- Schema development - In the first stage, the architects develop a set of spatial rules, referred to as the design schema. These rules are encoded into a user-friendly webbased Group Form design tool that allows non-experts to design their own residential units.

- Design development - In the second stage, end-users use the Group Form design tool to develop their own designs. The design possibilities are constrained by the architect's spatial rules embedded within the tool. Multiple users can work on their designs at the same time within a single site over a specified limited period of time. Each participant can see their neighbours' designs emerging over time, and various mechanisms allow multiple conflicting design decisions to be negotiated through online interaction and collaboration.

- Detailed design - In the third stage, the architect takes over the final negotiated design and develops it into a detailed design proposal.

The tool is a web application written in Processing language. It uses a client-server architecture to host all the design data and connect homebuyers so they can edit the designs. Each homebuyer makes changes to their designs on their own machines, but as every homebuyer's system is connected to the server, all changes made by any homebuyers are visible to the others almost instantaneously. In addition, to differentiate each homebuyer, they are given an avatar where they can see each other in the system interacting with the design. To make the design more interesting, and especially to encourage communication between homebuyers, the design is fully open without defining a specific plot for any of them. In other 
words, homebuyers compete with each other and negotiate through consensus or through auction-style bidding to achieve their desired space within the site boundary.

This design approach uses a very different method to those of the systems studied earlier in this chapter. This is necessary in order to engage the homebuyers more deeply with the design process, directly manipulating the space themselves. The first step was to define a set of spatial schema rules that produce design variations but maintain sufficient constraints for it to be practical. A hybrid approach of cell selection combined with various other techniques is selected, and the homebuyers design their space using three simple steps (Figure 33):

- Step 1 - the site is divided into a cellular grid and homebuyers define the area of their house by selecting a set of cells.

- Step 2 - the homebuyers rotate the cells to connect the habitable space into one area.

- Step 3 - the cells are further divided into smaller cellular grids to define the walls so homebuyers can specify the insertion points for doors and windows.

The cell size and definition are developed through a number of iterations. The habitable room (grey part) area is set to be smaller than the size of the cell. The cell size is set to $8 \times 8$ meters, and the habitable room $6 \times 6$ meters. Once a homebuyer selects a cell, they can offset the habitable room area by pushing it into any of the four cell corners. In a second step, they can further tweak the dimensions of the habitable room area. The original $6 \times 6$ meter space can be resized in steps of 0.5 meters; with a maximum of three steps. Hence, in plan, the area of each habitable room could vary from 25 to 36 square meters. This allows homebuyers to easily define their housing unit by selecting a small number of cells, while simultaneously allowing them fine-grained control over the dimensions of each habitable room area. 
Cell definition
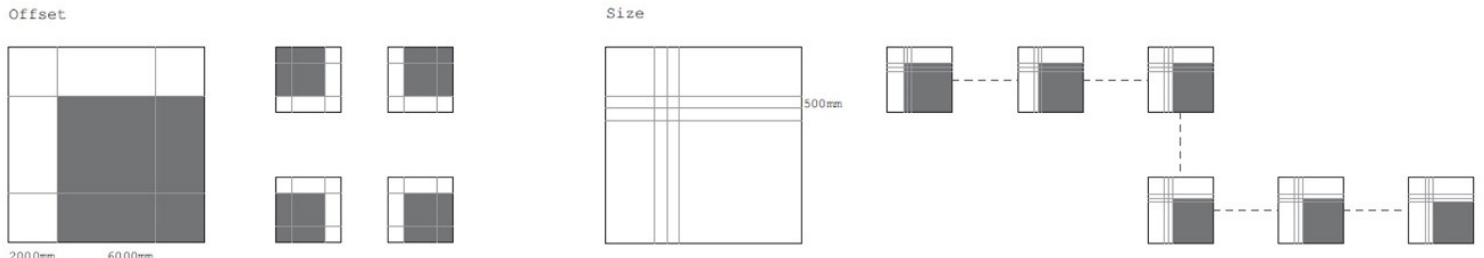

Plot negotiation
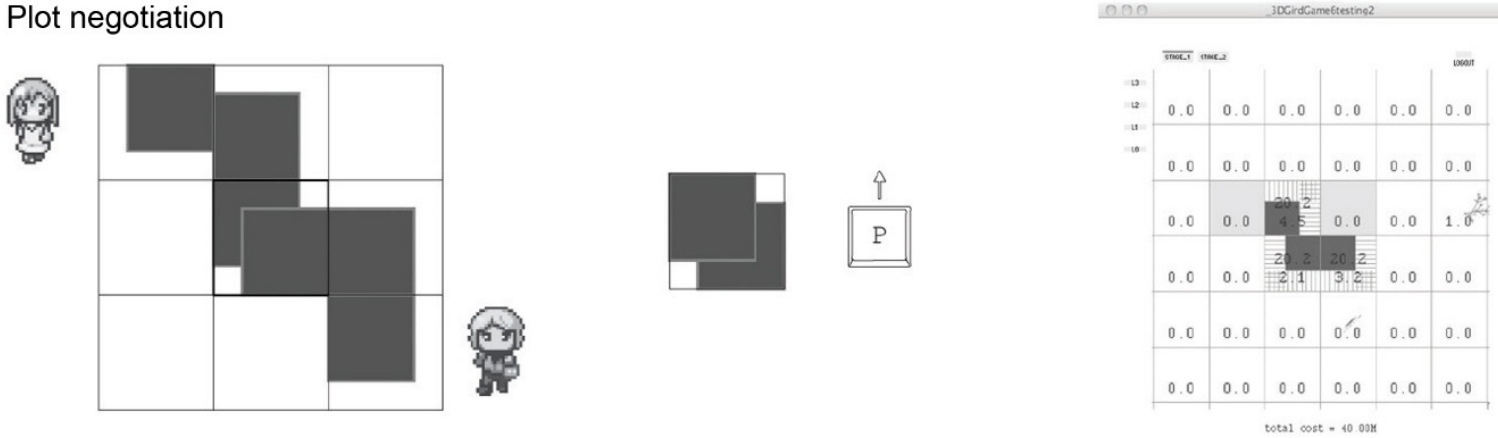

Figure 33 Cell definitions of Group Form (Ong, Janssen, \& Lo, 2013)

There are three key constraints defined to ensure reasonable housing units are generated (Ong, Janssen, \& Lo, 2013):

- Gravity - this constraint ensures there are no floating cells. The homebuyers can only select cells on the ground or on top of existing cells.

- Connectivity - subsequent cells can only be selected adjacent to (including above and below) the already selected cells by the same homebuyers.

- Size - the number of cells that can be selected is limited to four, so homebuyers do not over-select and design an over-sized house.

Once the cells are selected and the habitable areas connected, homebuyers move to the next stage to define the more detailed components, doors and windows. The walls are divided into square 'bricks' measuring $0.5 \times 0.5$ meters. Windows and doors can then be inserted into the walls by specifying the insertion point in this wall grid. Figure 34 shows screen shots of a homebuyer's interaction with the Group Form tool. For clarity, this scenario contains only a single homebuyer. The top three screen captures show step 1 and step 2 , in which grid cells are selected, and habitable room areas are defined. The bottom three screen captures show step 3, in which windows and doors are inserted. 
Design process of a homebuyers

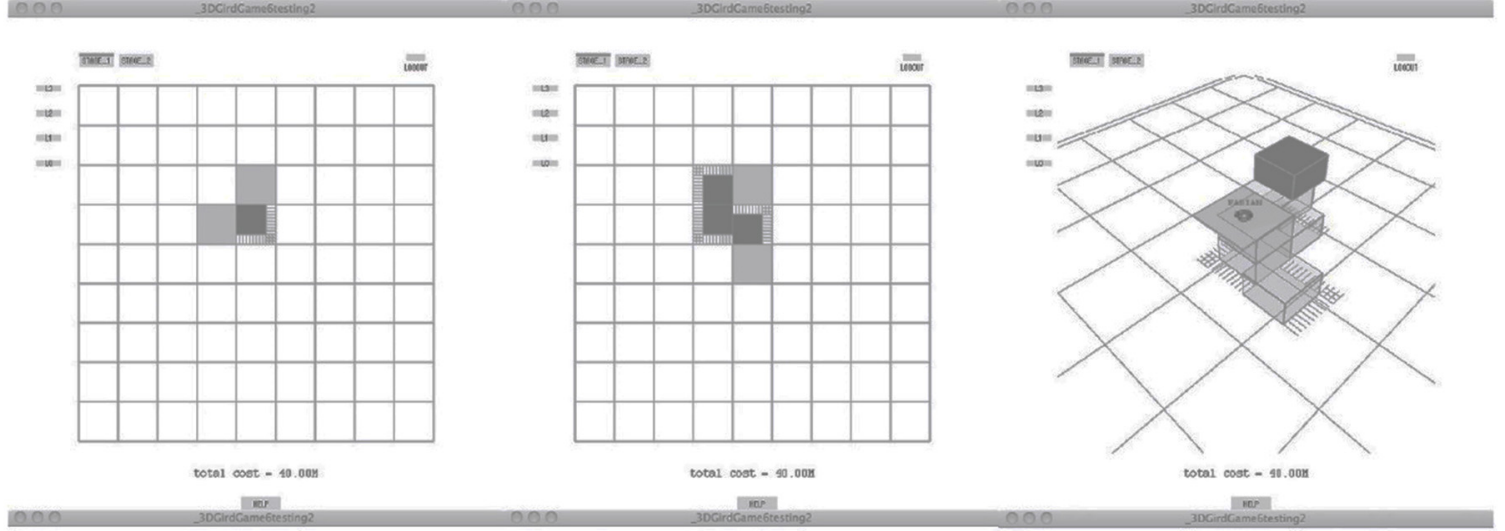

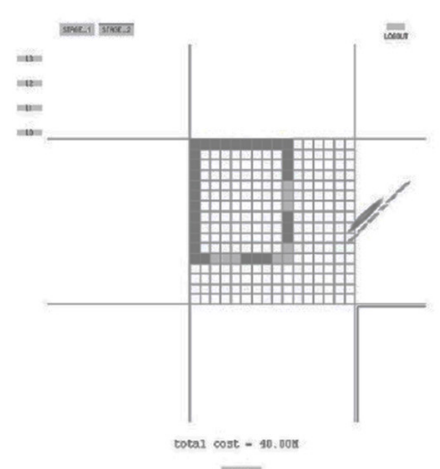

(n)

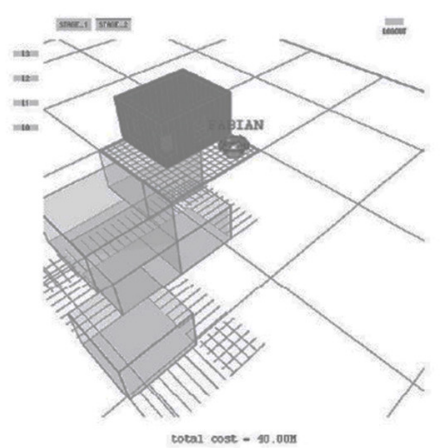

(n)

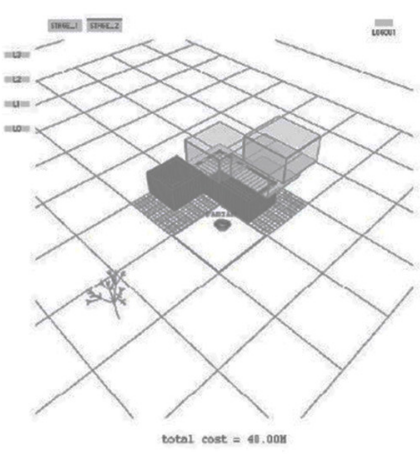

tate

Figure 34 Snapshot of the design process of a homebuyer with a video available online on Vimeo (Ong E. H., 2011) (Ong, Janssen, \& Lo, 2013)

A simple experiment tested the actual behaviour of the homebuyers while they collaborated in the Group Form system. Since available participants are limited, architecture students were invited to join the participatory process. They were each given a character card with a defined role based on age, gender, budget and occupation to role-play. Further details such as the relationship with other characters were also added to encourage more communication among the students and to have the characters they role-played living near or away from each other. The Group Form system does not have a communication platform, so the experiment was held in a room with all the students each interacting with their Group Form system. All communication is verbal and observed. The outcome was a complex and spatially rich arrangements of cells (Figure 35). It was very close to the expected outcome that the project was trying to achieve. The students also provided positive feedback regarding the design process and outcome. During the process, the students were asked to write down their aims, if they managed to achieve these, and if they are satisfied with the outcome. Their 
feedback was positive that the design process could enable the characters they role-played to achieve their desired outcome.
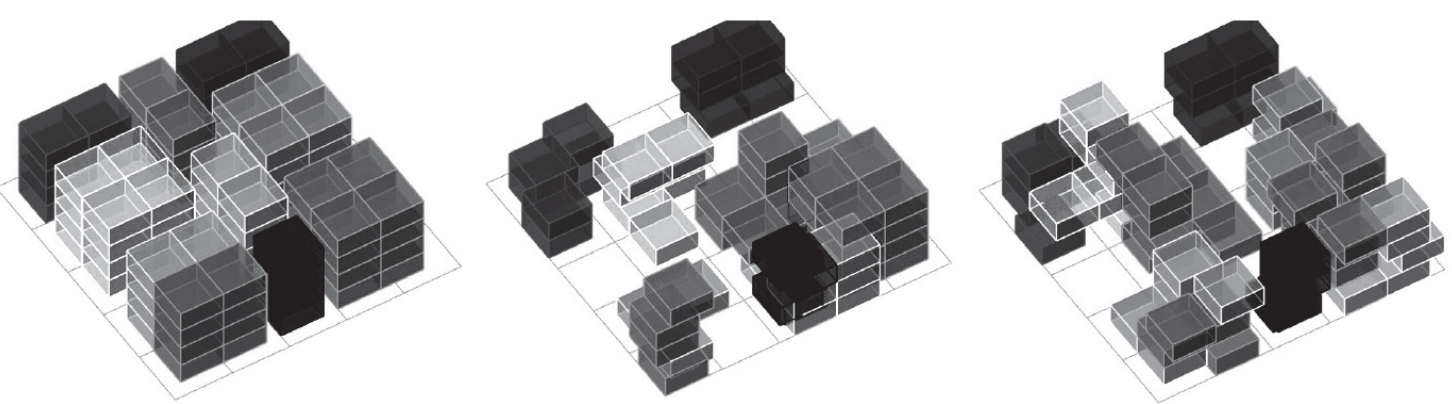

Figure 35 (left) current situation and worse outcome possible, (centre) experiment outcome, (right) expected outcome (Ong, Janssen, \& Lo, 2013)

As the project was a short master's thesis, the details of the housing design were not as extensive as the previous examples. The final outcome was developed into a design plan in a top-down manner by Fabian (Figure 36) to show the possibility of a practical outcome. However, the experiment has provided valuable observations regarding homebuyer-tohomebuyer interactions and how architects can play their role in this collaboration process.

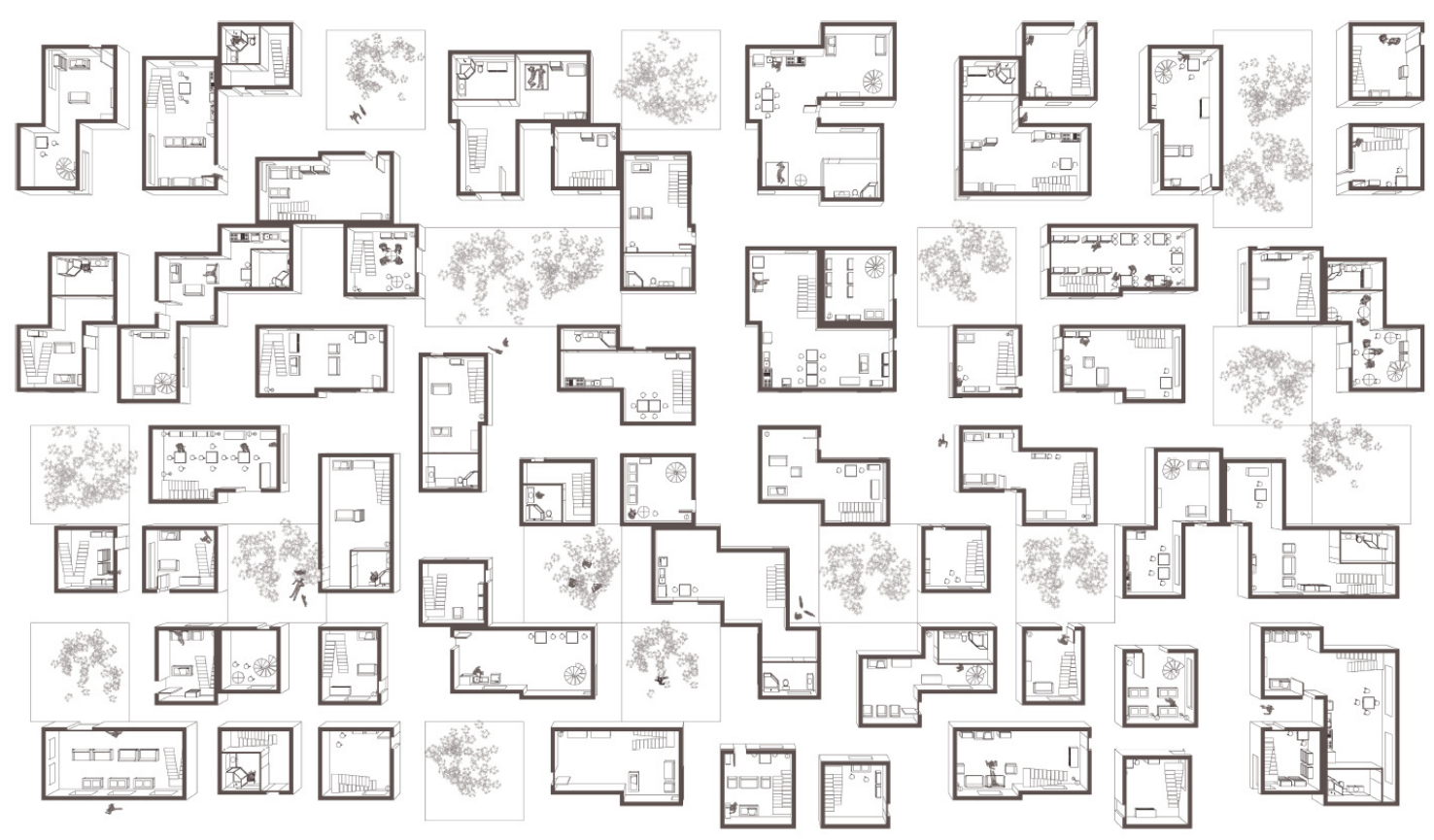

Figure 36 Spatial collaboration outcome generated into a practical plan (Ong, Janssen, \& Lo, 2013) 
Firstly, as the design is much more open, there is a need to define more parameters to facilitate communication among homebuyers and prevent unnecessary conflicts. The auction-style bidding proved insufficient.

Secondly, additional feedback can be provided to help homebuyers make more accurate design decisions. Building information such as energy consumption and daylight levels can also be incorporated. The family profiling used in previous systems to reflect whether homebuyers are fulfilling their needs is not included in this system.

Thirdly, the design approach can be further developed to bring about more flexibility with the interior spatial layout.

In conclusion, this project was an initial investigation reacting to an identified gap in existing design practices. By applying a suitable design approach, housing design can be simplified for homebuyers who can then manipulate the housing design directly, without hindering the final outcome. This can generate results that architects may not have thought of; developing the third order of synergy.

One very interesting factor arising during the experiment was that all participants were very excited by the game-a-like outlook of the interface. Participants' interactions with the system demonstrate a need for instant feedback, simple interaction, and conflict resolution, and encourage collaboration and constant communication and exchange of information. This is a great opportunity to use game logic and design method to enhance the participatory process in the housing context. The next chapter develops the idea of using gamification as a method for this housing design participatory process. Further analysis of games is undertaken to understand how game interfaces are designed to encourage the interaction of the players, how game mechanisms can be used for education and to simulate the real world and how game logics and theory can facilitate competition among players. These can provide insights to the framework of this research. Below is a table expanded from Table 4 that summarizes the systems studied in this chapter, including the initial experiment with Group Forming. 
Chapter 3 | Towards a Technological Mass housing Synergy

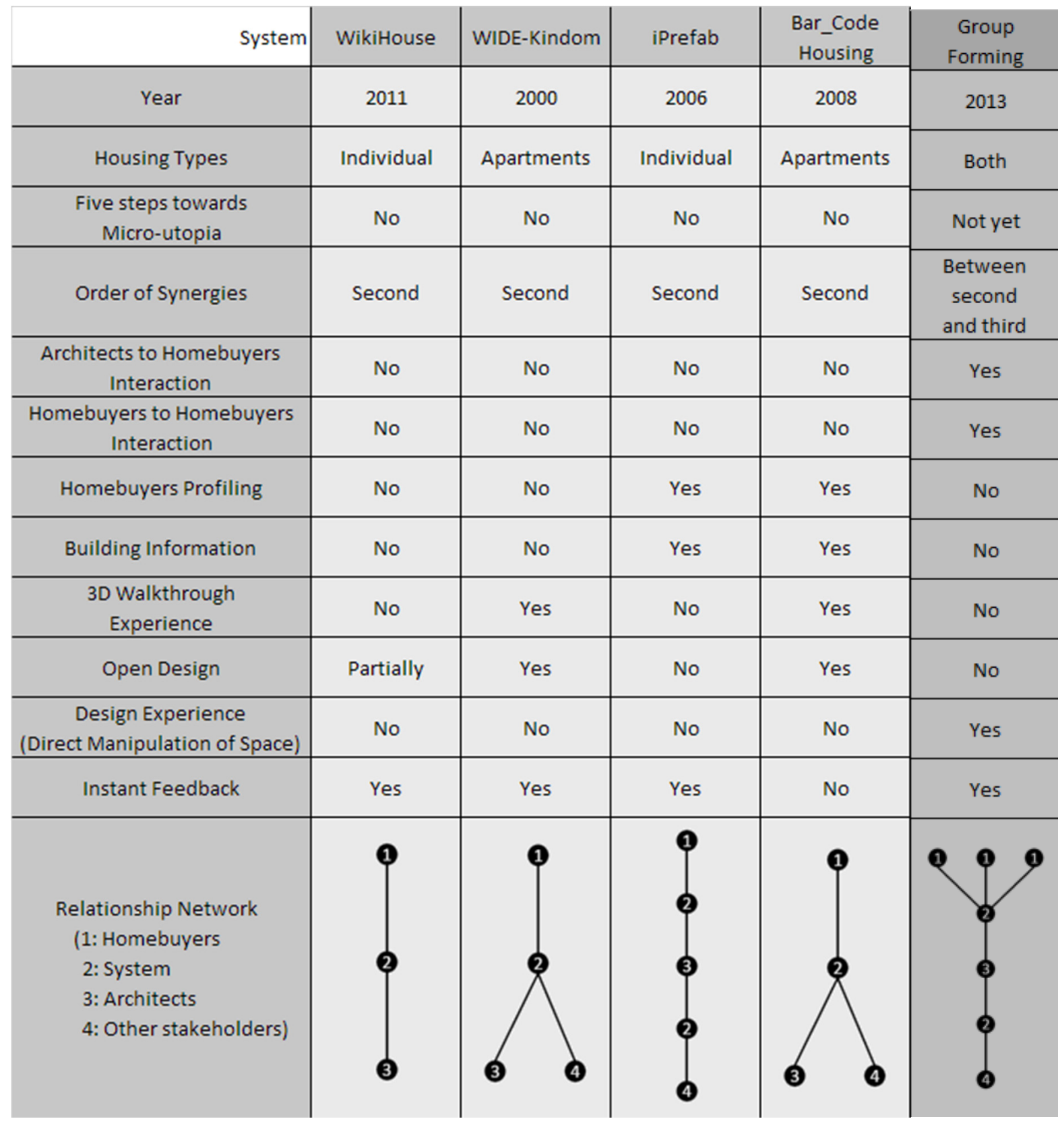

Table 4. Summary of the systems studies in this chapter 


\section{Chapter 4: Formulating the Methodology}

If you make a game about something that matters, your "players" will want to participate in that larger discussion. If you genuinely make that participation meaningful in the game, it can also be meaningful in real life.

- Ken Eklund -

(2014)

So far, this research has established the importance of enabling homebuyers involved in the design process of apartment buildings to collaborate with one another, and with the architects. Next, the capacity of technology to handle the participatory design process is demonstrated and reviewed. A research gap is then identified based on the study of the technology and how the existing tools are insufficient for handling the ideal participatory design process. Just before this chapter, an observation from a previous experiment provided an initial concept for the direction of this research, which proposes gamification as the strategic response to the research objectives. The quote above by game designer Ken Eklund (Mufson, 2014) after he created FutureCoast, a tool to inform the community about Earth's shifting climate using gamification, inspired this research to adopt the same method to encourage the participation of the people (in this case, the homebuyers). This chapter aims to strengthen the proposition by introducing the characteristics and functions of gamification, showing its potential to bridge the research gap as a methodology.

This chapter consists of three main sections. The first goes into the details of gamification; its meaning, its application in other industries and its successes and justifies how this is the desirable research method for this research. The next section further clarifies how gamification is integrated into the research process and explains the methods used to meet the objectives of this research. The third section presents the approach adopted for examining the research question. The research methodology is defined and the connection of the method to the literature review explained in detail. The desired outcome is to establish the target for this research and consider the appropriate approach for evaluating the test results. 
The table below gives an overview of the methodology framework of this research. The three sections described in the previous paragraph are the strategies, approach and methods respectively. The details of the participants are also explained in the last section of this chapter to provide the context for the data collection and analysis in Chapter $5 \& 6$.

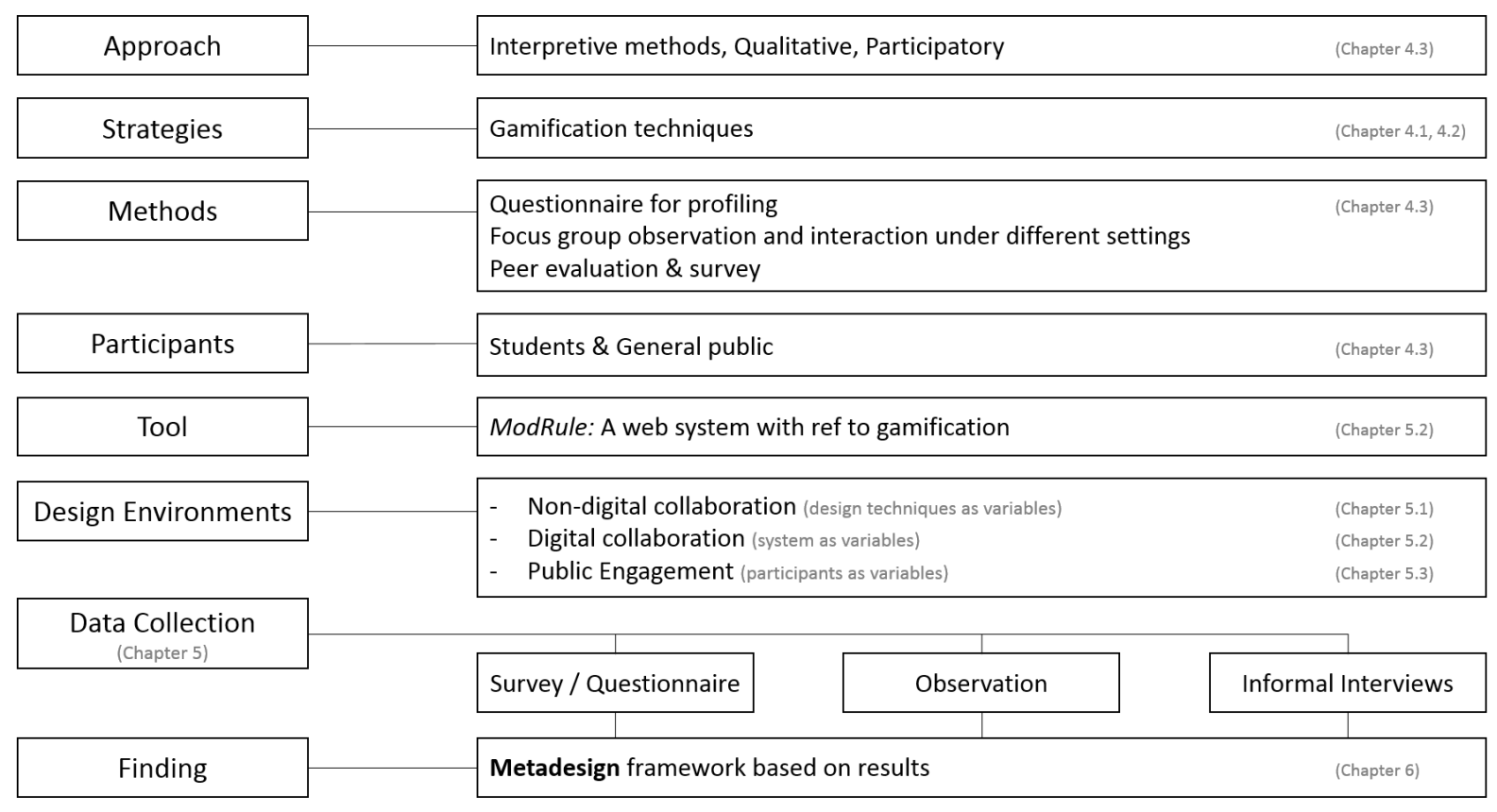

Table 5. Methodology Framework of this thesis research 


\subsection{Using Gamification}

In the previous chapter, the study of existing tools brought to light the factors necessary to realise the level of design engagement between homebuyers and architects. Developing a tool that includes all the elements could be a solution, but one yet insufficient to achieve the crucial third order of synergy. Wood argued that for synergies to take place, the system needs to be complex enough (Wood, 2007, p. 152); to the extent where the relationships are entangled with one another, each determining the state of the others. In this way, the architects' decision cannot be based solely on available data. They must be more intuitive and inventive, using their experience to guide an obvious course of action. He furthers his argument by using the example of the Mafia, whom he categorises as 'social organisation'; describing them as a synergistic organism very different from a corporation, as they have minimal managerial overheads and are capable of self-management. Wood believes that humans are smart enough to handle complex synergistic systems. This is a great advantage in housing design. He describes the crucial part of the complexity as coming from human emotions and maintains that most of the important synergy will remain 'elusive and intangible' (Wood, 2007, p. 153) as a result. In other words, one of the most important factors to bring about the levels of synergy is the participant's state of mind.

\subsubsection{PRACTICES OF GAMIFICATION}

This is where the research potentially leads to gamification. Johan Huizinga (1949 / 1938) argued that "playing" plays an important role in the development of societies and civilisation. Roger Caillois (1960, 1961 and 1964) later argued that the act of play might be more of an instinctive reaction to adapt to the surrounding environment. And Charles Coonradt (1984) is one of the earliest people to adapt gameplay practices within a workplace environment and explore the value of adding gameplay elements at work.

The use of the term 'gamification' can be dated back to the beginning of the last century. In 1912 Cracker Jack, a snack brand, started to give toys as a gift in their products (Aydin, Lo, 
\& Schnabel, 2014) but it is not until 2008 that the term is first documented, and widespread adoption only began in the second half of 2010. More recently, gamification is being promoted by marketing gurus and designers to describe a much more limited practice (Fuchs, Fizek, \& Ruffino, 2014). Several events and publications can be observed contributing to the definition of gamification. These include (ibid.):

- Gabe Zichermann's and Cristopher Cunningham's book (2011) which brings gamification into a business context, later started the associated annual conference Gamification Summit in San Francisco in 2011 and have held it annually ever since.

- Jane McGonigal's TED talk (Gaming can make a better world, 2010) about how gamers could save the world. Jane later wrote a book (Reality is Broken: Why Games Make Us Better and How They Can Change the World, 2012) showing how she brought gamification to corporations such as McDonald's and the Olympic Games organisation where she consulted on marketing campaigns based on alternate reality games.

Although gamification in this instance focuses on the domain of marketing to encourage more 'consumption', it describes the use of game design elements in non-game contexts (Deterding, Dixon, Khaled, \& Nacke, 2011). Definitions of gamification differ between people, and depend largely on their familiarity with this term. This research defines gamification as 'collectively productive'.

How gamification is connected to the 'state of mind' described by Wood can be better understood through Chou Yu-Kai's eight core drives of gamification (2015). In his book "Actionable Gamification: Beyond Points, Badges, and Leaderboards", he describes gamification as a form of behavioural design or human-focused design. This means systems that are not designed to simply get the job done but are optimized for human feelings, motivations and engagement. He believed that games, if not focusing on escapism, have the capability to engage people for long durations, foster meaningful relationships between people and, most importantly, develop creative potential among them; concepts with which this research stands parallel. The emergence of social and mobile games like Candy Crush and 
Angry Birds have shown that games are no longer just for youth but appeal to middle-aged executives and senior retirees alike (Chou, 2015). Moreover, gamification has already been adopted unconsciously by consumers in every aspect of our lives (ibid.):

- $\quad$ Marketing - the U.S. Armed Forces is spending more money on recruitment games.

- Work - Entertainment Software Association has $70 \%$ of its major employers using gamification to enhance performance.

- Healthcare - Nike uses gamified feedback to encourage their users to train above their personal fitness goals.

- Education - Beat the GMAT has used a gamified platform to increase the time students spent on the websites to improve their test scores.

- Research - A 15-year AIDS virus protein problem was solved in 10 days by gamers after researchers created the gamified online platform, Foldit.

- Design - Volkswagen uses its People's Car Project to attract the public to their websites and to contribute ideas to design the 'perfect car'.

The potential application of gamification is endless and, most importantly, targeted at engagement with the public; a means of which this research is specifically searching for. Charles narrows down the reasons for people's willingness to engage in such activities to the following five points (Coonradt , 1984):

- Clearly defined goals

- Better scorekeeping and scorecards, or rewards

- More frequent feedback

- A higher degree of personal control over processes and results

- Consistent tutorials and help throughout the engagement 
Referring to the table in the last chapter, these five points by Charles are also the factors identified in this research through the study of housing design systems. This provides a strong basis for this research to adopt gamification as the primary method for achieving extensive engagement between homebuyers and architects during housing design processes using computational tools within a virtual environment. The next section provides an in-depth understanding of gamification, Chou's eight core drives of gamification, the various game mechanics, theories and techniques that could be used for the study of this research and some of the risks potentially entailed.

\subsubsection{GAMIFICATION IN THIS RESEARCH}

Gamification is not turning everything into a game. The purpose of gamification is not to pull us out of reality but rather to find what is fun in an activity that requires collaboration and engagement. They are not necessarily the 'serious games' such as those used as training and learning environments in military and educational examples. The focus of simulations in serious games is on testing the abilities of learners and on improving their skill sets in a virtual environment similar to real conditions. Game theory, in contrast, is used to mathematically analyse decision-making 'strategies' or individual 'choices' (Kelly, 2003), and gamification may be helpful to improve collaboration on 'a choice' and encourage involvement in 'a strategy' (Kapp, 2012). Also, points, badges and leaderboards (PBLs) are irrepressibly penetrating every aspect of our daily lives in tandem with the growing use of social media. PBLs are one of the most common game elements. However, they are not sufficient with regards to what games and game design can provoke. The approach is to gamify a situation by thinking like a game designer, which is different to being a game user. When using a gamified system, a player is still considered a user (Aydin, Lo , \& Schnabel, 2014). The term 'play' used in the following text is not just about playing a game but includes using an application, or engaging with a system. 


\subsubsection{THE EIGHT CORE DRIVES OF GAMIFICATION}

Going beyond PBLs is what drives Chou to develop the eight core drives of gamification. Most gamers or application users do not play the games or use the applications for the PBLs (Chou, 2015). It is usually the presence of elements of strategy, a means to spend time with friends and family, or simply the challenges and excitement they provide. The PBLs are instead a bonus as part of the gamification mechanics. Chou categories the eight core drives as the following (also shown in Figure 37) (ibid.):

- Epic Meaning \& Calling

- Development \& Accomplishment

- Empowerment of Creativity \& Feedback

- Ownership \& Possession

- Social Influence \& Relatedness

- Scarcity \& Impatience

- Unpredictability \& Curiosity

- Loss \& Avoidance 


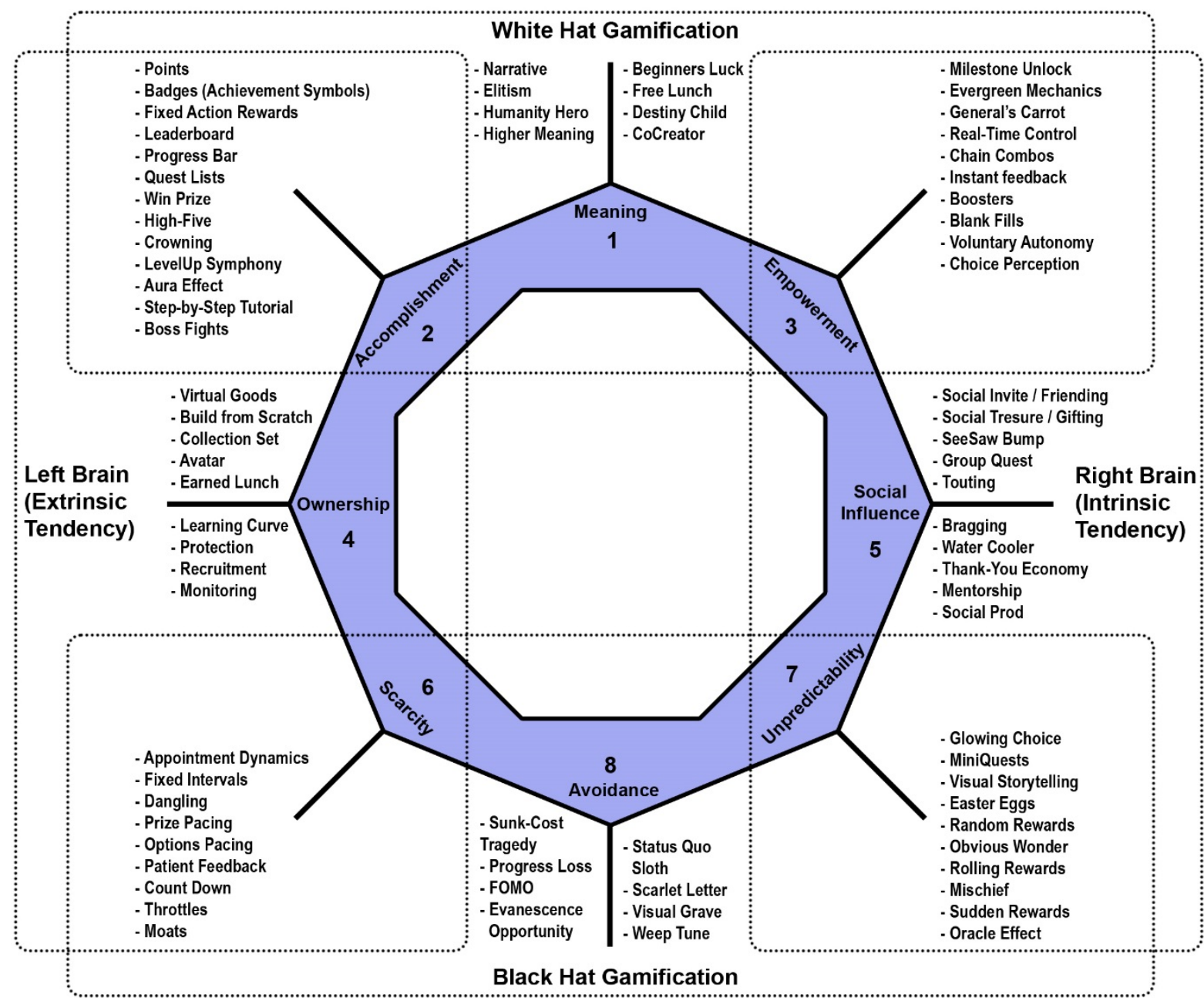

Figure 37 The Eight Core Drives of Gamification (Chou, 2015)

The first core drive is providing meaning to the person in play. This could be the 'chosen hero' to save the world - what many games are about. Or simply giving users a sense that they are contributing to something much bigger than themselves.

The second core drive is about progress. This is what PBLs are most focused on. The purpose is to inspire and keep users motivated to grow with the system; developing skills, achieving mastery, and eventually overcoming challenges which the system is trying to cultivate. This could be through gaining experience points to improve the game character and allow it to advance through the story narrative, or simply getting badges for various achievements like in mobile app, Foursquare, which encourages people to 'check-in' to place they go to gain points and get discounts for certain products. 
The third core drive is about pushing users' creativity through constant feedback. The idea is that users are not simply engaging with the system but are involved in a creative process where they constantly create and develop new results. These are monitored through feedback from the system or from the community within it, and the users can then make further adjustments. If the techniques are properly designed, like Lego bricks, the system can eliminate the need for constant new content and users simply entertain themselves through the creative process. This core drive is also what this research will maximise to engage homebuyers with a creative process.

The fourth core drive is where the motivation comes from giving users a sense of ownership of something, or of being in control of someone. This could come in the form of virtual goods or currency within the system. 'They' could also be a certain virtual character or avatar which users can customize as they like. The more time and effort users spend to accumulate 'wealth' or improve their 'character', the more they feel ownership over a process, project or organization and thus, the more immersed in the system they become. This is what this research hopes to instil in homebuyers during the participatory design process.

The fifth core drive is to gather people together to bring about social elements such as mentorship, social acceptance, social feedback, companionship and even competition. This can be easily seen in social media such as Facebook, Twitter, and Linkedln. This drive can induce people who want to share and learn from everyone to build up a community that they can closely relate to. Making use of the fast-growing internet community, people are so connected now that social activities can be easily organised and promoted. Corporations are even using them to connect to people and promote their products. Websites like Kickstarter are also using online connections to foster interest in new products and projects and draw crowdfunding from the people. This core drive is an important factor for this research to bring about social interaction and collaboration between homebuyers.

The sixth core drive is branding something as extremely rare or exclusive, like 'limited edition' to bring about a sense of urgency to the people towards the product. It could also be 
making a product available only after an initial restricted time-period. Facebook was originally only for Harvard Students; later extended to a few prestigious schools. By the time it was released to the world, everyone was so eager to join it became a phenomenon. Such 'Appointment Dynamics' or 'Torture Breaks' are also methods that many games use to encourage players to spend more time with the games. But in a non-marketing context, to integrate this drive into a design system, there is also a need to refer to Jane McGonigal's 'urgent optimism'. That is to drive the people to act to solve a problem with a sense of urgency and the belief that they have a reasonable hope of success (McGonigal, 2012).

The seventh core drive is to attract the attention of the people by appealing to their curiosity. This can be observed in movies where an unpredictable plot draws more viewership to the ending. This can also be related to the third core drive, making the system open-ended so users are drawn to engage with it - using their creativity to develop an outcome that they cannot expect. This research anticipates that if this core drive can be applied not only to homebuyers but also to architects, the unexpected outcome generated by the homebuyers could encourage architects to use the system more regularly.

The last core drive capitalises on people's fear of loss or missing out on something of value. This can be related to the sixth core drive but works more towards preventing people from stopping using the system. Instead of rewarding, this core drive gives users an impression that what they have contributed will be wasted unless they continue to use the system.

Every core drive serves a different purpose towards gamification, but they are also related to one another. Chou further groups them into four categories based on the nature of their motivation (Chou, 2015):

- Left brain (Extrinsic tendency) drives vs Right brain (Intrinsic tendency) drives

- White hat gamification vs Black hat gamification 
In Chou's categories left and right brain does not literally mean the actual topography of the brain, but rather a symbolic differentiation between the functions of the two sides of the brain related to the purpose of the core drives. Left-brain core drives are factors that rely on tangible returns to motivate; right-brain core drives depend on self-motivation. This classification is important in designing with gamification because rewards or extrinsic motivators soon get exhausted and users lose interest rapidly (ibid.). This classification describes clearly where methods fall within the respective categories and helps prevent them from being too one-sided.

The next classification is 'White hat gamification' vs 'Black hat gamification'. This is more straightforward as white hat simply means positive motivations. These include making users feel a sense of achievement through skill mastery or a sense of responsibility, or higher sense of meaning from the role and tasks given to the users in the system. On the contrary, black hat means negative motivation. These are usually a sense of fear of losing something or uncertainty about progress, or trying to achieve something that might not work, yet still driving users to consistently work with the system.

Last but not least, there is a ninth core drive which Chou does not include and that is sensation. Compared to the eight core drives which bring motivation through psychological means, this core drive is the emotion or preference in people driven by physical feelings through the five human senses.

Recalling Wood's five steps towards micro-utopia, the first step is to break through the psychological barrier among users. Since this research references Wood's model to enhance the interaction between homebuyers and architects using a digital system, the focus is on the psychological factors. However, this does not mean that this core drive is negligible. Instead, this core drive will be taken into consideration when necessary during the tool development. 
Applying gamification is not just engaging people through PBLs but more about motivating and driving people to develop and socialise within the system. By connecting the dots, it is easy to see that these core drives are much related to Wood's description of levels of synergy. Core drives motivate people to engage with a certain 'object' and through the process, develop growth, interaction and collaboration between them. This research simply defines the 'object' as the housing design participatory system for specific users such as homebuyers and architects. This provides an extensive guideline for contemplating the gamification structure of the housing design system. It need not be necessary to include all eight core drives but to vary the degree of the respective cores depending on objectives.

To provide a better illustration of how they can be applied, Chou analysed some games and applications based on the core drives as shown in Figure 38.

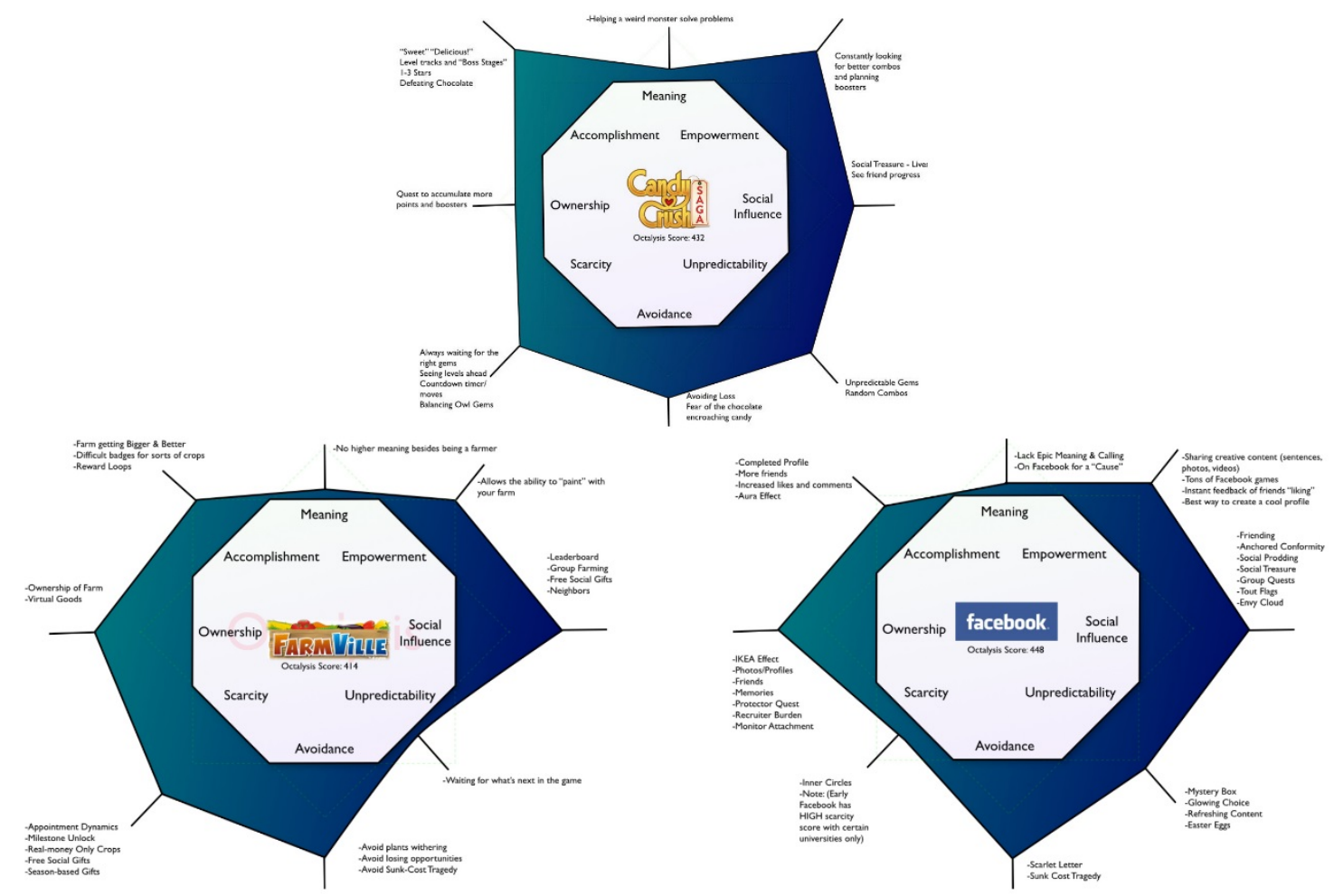

Figure 38 Profiles of some social games and application based on the eight core drives (Chou, 2015) 


\subsubsection{RULES OF PLAY: GAME MECHANISMS}

Although the eight core drives show how to motivate people to use a system, the approach to designing such a system is yet unclear. The most direct way is to examine how games are designed. Katie Salen and Eric Zimmerman wrote a book (Rules of Play: Game Design Fundamentals, 2003) to describe the fundamentals of game design. This research does not go into too much detail about the elements of game design, but studies the framework of the book around the idea of game design schemas. This acts as a lens to magnify the understanding of the analysis and creation of games, which consist of three primary schemas: Rules, Play and Culture as in Figure 39.

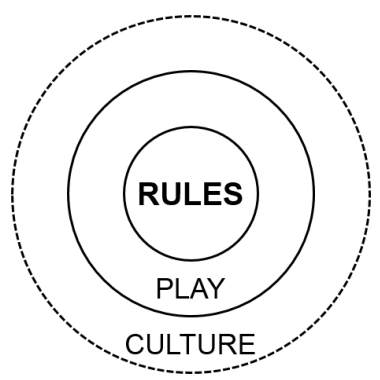

Figure 39 The three primary schemas (Salen \& Zimmerman, 2003)

The Rules schema is the formal element of the games which focus on intrinsic mathematical structures. It is a subset of Play schema, the experiential element which emphasizes the player's interaction with the game. The Rules are what define the Play but the Play constitutes communication processes which might not be controlled by the rules, and form the bigger domain of game design. The whole scope of game design is however, the Culture schema, the contextual element which highlights the cultural contexts of the game. It is this schema which sets boundaries for the types of gameplay and the meaning and value that the system provides. In this research, the context is the housing design process. The eight core drives of gamification is more relevant to the Play schema here. This leaves the most important part of game design, which could help identify approaches to developing a gamified tool of engagement. 
Gamification development can adopt the three levels of game design rules:

- Constitutive rules are first. These are the abstract, core mathematical rules of a game. Putting it into a digital design context, this represents the essential algorithm within the system structure. They do not explicitly indicate how the users should enact these rules, but they reflect the underlying framework of the system that informs the operational rules, the second rules.

- The operational rules are the main 'rules of play' that users must follow when they are interacting with the design system. These rules directly influence the behaviour, input and output of the users and are usually printed out as instructions or manuals.

- Lastly, the third rules are the implicit ones. These are the 'unwritten rules'. They are the etiquette and behaviour of the users that usually go unstated. In game culture, these are well established as a form of 'sportsmanship'.

This provides another layer of guidance for the development of tools of engagement. How these three rules are used for the tool development in the context of mass housing is further explained in Chapter 6. Like game programs, digital tools of user-oriented design are multi-layered, complex objects. Specifying which aspects of a system belong to each type of rules is usually unclear. However, this three-part rule framework can be applied to better understand the abstract formal system in achieving meaningful interaction through gamification.

\subsubsection{TOUCHING ON GAME THEORY}

So far, this chapter has touched on various aspects of gamification and considered the possible ways of integrating play elements into a system and drawing the attention of users to engage with it. However, these factors focus only on system development and users' connection towards the system. This research has stated that, since the design collaboration involves interaction between the homebuyers, some form of conflict of interests will exist. This differs from the competitions between players in games. The outcome from the housing design system proposed in this research is not a simulation. The homebuyers are designing a housing 
unit which they are going to live in and pay great amount of money for. From personal experience, players in games can be so dedicated to the gameplay that they use harsh words or ban players whom they deem 'noob' (inexperienced players). In response, fellow players will say "It's just a game", and let the matter go. However, for the practical courses of action occurring in the system of this research, the conflict will be less-easily resolved. This can be observed in the Group Forming system studied in the last chapter. Certain strategies must be outlined to bring about harmonious negotiation between homebuyers instead, which may then progress into a collaborative effort.

In the world of economics, these monetary conflicts are common entities. The general concept of economics was put together by Adam Smith, who is also known as "the father of economics" (Blenman, 2017). He is the key figure who promoted free trade and that selfinterest or individual ambition will inadvertently serve the common good. However, that is only effective if the involved parties have different interests and they compensate for the needs of one another. In the case of this research, the architects are serving their own interests to design a better place to live and the homebuyers have their own desire to obtain a conducive environment and call it their home. This relationship allows a natural phenomenon that guides it towards the direction of efficiency. Game theory, on the other hand, is the study of human conflict and cooperation within a competitive situation (Investopedia) which in this research is the relationship between homebuyers. This theory is developed by mathematicians John von Neumann and later extended beyond zero-sum analysis by John Nash (Chang, 2015). They laid the groundwork for formal expression and a framework for investigating the various decisions made in competitive environments. Like gamification, game theory references a game situation in which individual members are involved in a situation with similar rules and consequences. Its application can suit a wide range of situations, even for design collaboration between homebuyers in this research. There are some conditions to be met for game theory to apply (Investopedia):

- Identity of every individual involved 
- Preferences of each individual

- Knowledge they have with respect to the situation

- Every strategic course of action they are able to take

- The impact of every decision on the outcome

There are various other conditions or assumptions depending on the situation, but these are the basic conditions to enable explicit analysis of the various possible choices one can make to achieve the potential outcomes. When compared with the necessary factors identified to develop a collaborative design system, they are wholly related and thus one of the main reasons for this research to find it appropriate to adopt game theory as the method to resolve possible conflict in the design process. Game theory is mainly used by researchers focusing on how groups of people interact.

Game theory can be broken down into two main branches: cooperative and noncooperative game theory (Levine, n.d.). Since the aim of this research is to achieve collaboration between homebuyers, it focuses on the cooperative aspect of game theory. The particular concepts which correspond to this research is the Non-Zero Sum game and Nash Equilibrium. The mathematical definition of a non-zero sum game is that the sum of all the gains and losses by a group of players is not always equal to zero for every possible outcome (Wright, 2001). In other words, there are possibilities of a win-win outcome where both parties meet their desired interests, or lose-lose outcomes where no one gains anything.

In the context of this research, if design choices and outcomes are structured properly, homebuyers might decide to work together and collaborate to achieve the best win-win outcome. That is also where the architects play an important role; facilitating the communication to encourage such design collaboration to take place. Integrating the motivating factors of gamification with game design rules and game theory might provide 
the means to enhance the capability of a digital design system to achieve the desired design collaboration between homebuyers and architects.

\subsubsection{RISK IN USING GAMIFICATION}

Although gamification seems capable of solving problems or even changing the world (McGonigal, 2010), it is not altogether popular. The book 'Rethinking Gamification' by Mathias Fuchs et al. (2014) collates a collection of articles that looks at the controversies of gamification. In it, they used lan Bogost (2011) to highlight the frustration as he claimed that gamification does not have any relationship with the design of games and is used mainly to exploit consumers. He condemned gamification as he considers it to devalue the practice of game design and reduce the idea of 'play' to simple stimulus-response experiences. The fact that games and video games have been trying to bring the meanings of play in a digital culture to greater heights, 'gamification' is considered a bad word for those involved in the gaming industry (Fuchs, Fizek, \& Ruffino, 2014). However, the fact that gamification does work in many other industries shows it is here to stay. The book proposes to keep the word and to change its meanings and the ideas associated with it. The term 'gamification' retained, but its meaning restricted to its real intent to convey political statements, artistic values, educational content or any sort of unconventional messages. This research agrees and therefore reiterates that gamification is not about games at all but the study of interactions between people and with a system. This provides the appropriate precautions for this research. Ultimately, this research is about homebuyers, architects and the housing design process, and to study the possibility of bringing them together using gamification. It is not just about creating a tool with gamification elements.

\subsection{Integrating Gamification into a Collaborative Design Tool}

Having gained an understanding of gamification, the next step is to examine how this can be integrated into a digital collaborative design system. Referring to the identified gap, the aim is 
to use gamification elements to bring about design collaboration between homebuyers, and with architects. The platform must allow homebuyers to directly manipulate the various architectural components easily, and use the output design effectively for the design exchange and communication among parties to achieve a third level of synergy.

\subsubsection{ENHANCING INTERACTION WITH THE TOOL}

The first objective is to examine how gamification could play a role in enhancing homebuyerinteraction with the digital tool. The eight core drives provide the necessary viewpoints that go beyond the design of interface and human-computer interaction $(\mathrm{HCl})$. The context of housing and the need to acquire a home that suits the homebuyer also corresponds to the application of the core drives.

Looking deeper into the principle of the core drives, note that the method is not to incorporate the eight cores drives into the design system but to identify the elements of housing design that could be integrated into the design system to bring out these qualities. One such element could be simply giving the homebuyers more freedom to manipulate the space, for example by adding their own architectural components and materials. This gives homebuyers the opportunity to use their creativity to design their own living space but is only possible if the design process is simplified enough.

\subsubsection{SIMPLIFYING THE DESIGN PROCESS}

This leads to the second objective: To simplify the design process in a way that homebuyers can interact with the system with ease. The 'rules of play' studied earlier respond to this need. The aim is 'simplification', and it is about breaking down the design process into simple steps that enable homebuyers to get 'hands-on' more easily. The 'rules of play' provide a methodological direction for this approach.

To avoid the trap of developing the design tool into a game, the rules are adopted with a practical reality in mind. The constitutive rules can be used to prepare the structure of the design; which is to translate housing design into simpler geometry or formulas. This can be 
associated with parametric design where the design is based on parameters, rules and constraints, but simpler in this context. The operational rules must work closely with the core drives so that the homebuyers are motivated to use the design system. The implicit rules are a challenge as the understanding of homebuyers is required to make the system work. This can be developed overtime; observing the homebuyers interacting among each other and with the architects within the system to understand how these rules can be generated. There is, however, a provisional method that can provide this research with an initial approach which is explained in the next section.

\subsubsection{PROMOTING COOPERATION}

From the study of the Group Forming tool, a simple bidding system is used to negotiate spatial ownership among the participants. The result achieved was more competition than collaboration. Since everyone knew from the start that the richest person will win, there was no motivation for communication among participants. This is where game theory comes into play. The third objective of promoting collaboration among homebuyers requires more than putting the homebuyers together within a system. It is necessary to adopt a method to manage the design communication.

Game theory provides an observable structure to examine how the design system can be developed to mediate communication among homebuyers. By touching on the cooperative nature of game theory, this research can identify the factors necessary to motivate homebuyers to work with each other, even with their heterogeneous backgrounds and diverse needs. This may include methods to set design parameters and the engagement rules. The aim is to ensure that while the homebuyers are interacting with each other in the design system, they focus not only on their own interests but also those of other homebuyers and, more importantly, work together for the living community as a whole. 


\subsection{Studying Design Tool with Focus Group}

To understand how homebuyers will interact with the digital collaborative design tool, and study the extent of the development toward the design outcome, a qualitative approach is required. Instead of using specific data figures to determine an argument, this research looks to capture the experiences and views of the homebuyers towards the collaborative design process using a digital tool. Since multiple perspectives are desired for this research study, a 'focus group' is selected as the main method of data collection. It is very different to questionnaires and interviews since the participants are part of a discussion with an experienced moderator (in this case, the researcher). It does provide more effective qualitative data for analysis.

The studies made during the tool development process adopt the focus group technique as the research method. This method is frequently used in social science studies to take advantage of the interaction between participants to bring about quality perspectives on certain subject (Carey \& Asbury, 2016). Although this research is about architecture design and digital computation, it is very much related to the study of social interaction between homebuyers and architects. The data generated through the focus group method are usually interactive and appropriate for qualitative analysis. This is very suitable for this research as the data are usually illustrated extensively (Wilkinson, 1998). However, focus groups are usually small in sample size so may not be sufficiently representative. More specifically, a focus group is around six to twelve individuals who are strangers to one another but with some commonalities, such as experiences or knowledge, discussing a specific topic with a researcher (Asbury, 2016). As the members of the focus groups are usually well-informed individuals, although the number is small, their input is enough to bring about the in-depth understanding of design interactions between participants that this research requires for the tool development.

This research includes two focus group studies; one without using any digital tool and one with the tool developed for this research. The aim is to have a comparative analysis 
between the two studies to examine the extent of the advantages or disadvantages the tool provides for the homebuyers.

Another study is implemented with the public to obtain additional feedback regarding the adoption of a design tool for housing design. This study makes use of questionnaires and simple conversation exchanges with the individuals.

With the method established, the next chapter dives into the development of the tools and studies whether adopting such a method provides the means to enhance the design participation process. The table below outlines the three studies that are carried out in this research. The three studies varied regarding the participants and the environment. They are set up according to the needs of the investigation. The data collected are analysed to generate the method for the next study. Chapter 5 will demonstrate how each study is performed in details.

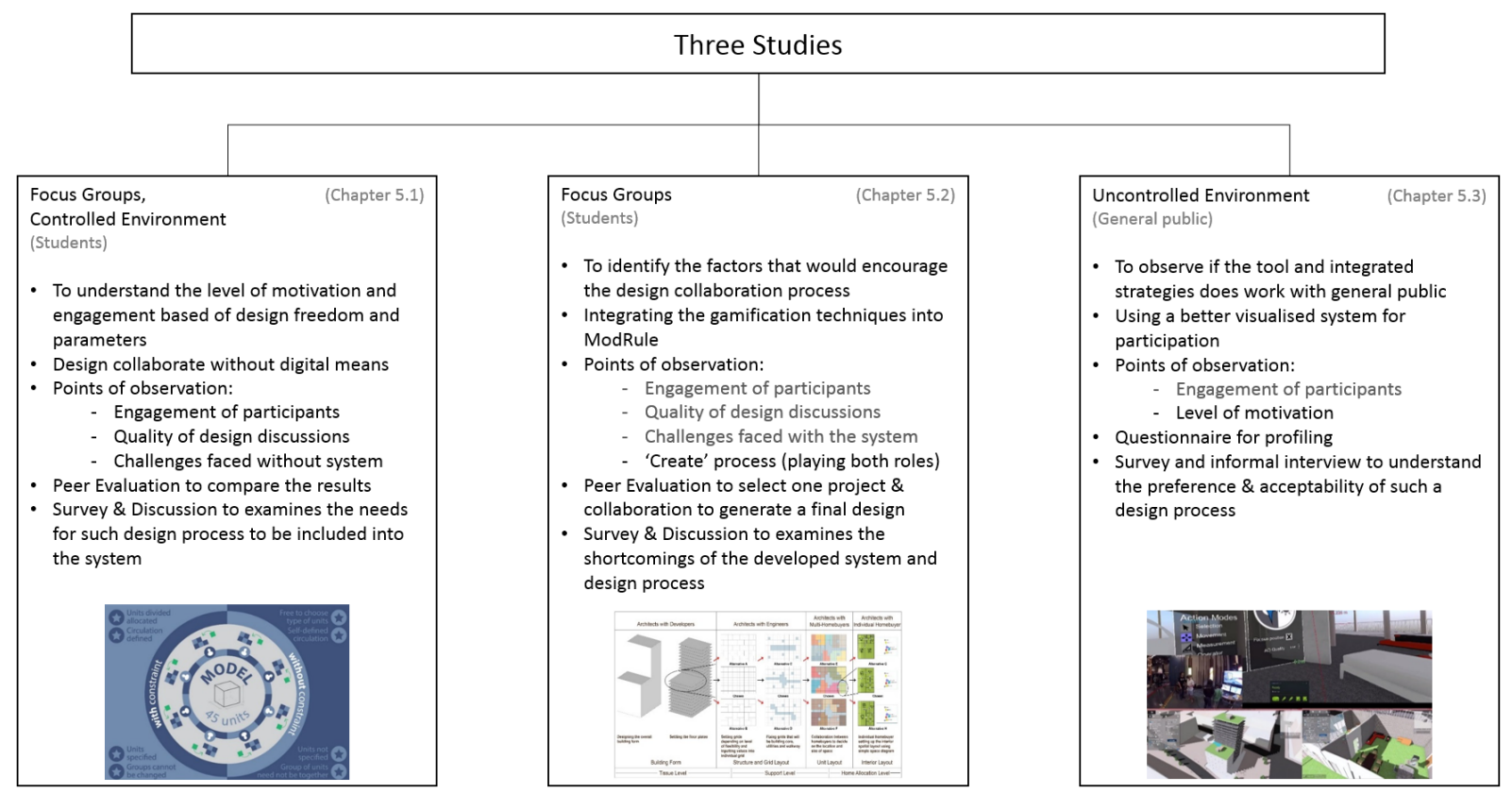

Table 6. Outline of the studies carried out in this research 


\section{Chapter 5: Enhancing the Design Participation Process}

Making simulations of what you're going to build is tremendously useful if you can get feedback from them that will tell you where you've gone wrong and what you can do about it.

\section{-Christopher Alexander-}

(2006)

Having gained a better understanding of the needs of homebuyers, the gap in existing digital systems to provide for these needs, and the possible methods of bridging the gap, the next steps are to develop a tool to test the position of this research. To Alexander, making a simulation and obtaining feedback through experimentation and observation with real participants is the best way to understand if the proposed theory works, and what potential and limitations exist in the method.

This chapter goes into the details of the tool development and outlines the various stages of the research as it explores the influence of gamification on the design process. There are four parts to this development. The first is a pre-development design process experiment to figure out how gamification can contribute to the design process without a digital tool. The second part is the creation of a tool based on the observations of the first experiment, and includes a study to investigate if the tool works. The third part enhances the tool, accounting for any missing functions that may hinder the success of this research. The development and study of the tool provides insights that enable an elaborate framework with which to engage homebuyers with the design process and architects using digital tools.

\subsection{Pre-development}

To find a means of achieving collaboration between the various stakeholders and the architects is not simply to create a digital platform for them to communicate through. That could easily be accomplished with existing technology or through a social network platform. The main objective is to understand how mass housing design can be 'simplified' into simple rules and parameters so the stakeholders can engage in the design process easily. Considering this 
objective, there are sub-questions to address. Since the focus is on the homebuyers, there are some social aspects to be examined. By setting the parameters optimally, the platform could achieve sufficient balance for there to be fair play among everyone. The controls must be optimized so that the collaboration of the occupants does not dictate the design too much, causing the design to lose control; yet not overly constrained, making participation meaningless. In addition, these parameters must be simplified to allow anyone to easily understand them and so ensure the possibility of collaboration. Another sub-question is how to enable the stakeholders to change their decisions at any point of the design process. For an open system to work, the goals and criteria of the occupants must be incorporated. This must also be done in such a way that participants can reach a consensus at some point. Thus, the modelling process in this case must be 'free', so that it can be discussed, negotiated, and changed during use. This is where the digital platform comes into play. As the modelling process has become part of the design process, with changes becoming constant throughout, the speed and efficiency of computers is necessary to organize the unstructured collection of possibilities.

To a great extent, these also revolve around the parameters used. The organization of the design options must be controlled in an optimized manner so that it is easy to manipulate, yet manageable at the same time. In addition, the parameters must also be controlled so that the design is controlled, yet with sufficient freedom to achieve individuality for the occupants. Essentially, this research focuses considerably on design parameters and workflow processes that can encourage collaboration and enhance data management.

\subsubsection{DESIGN STUDIO PARTICIPANTS}

To further understand the possible problems that may occur in collaborative design, a design studio was set up to explore the process without any digital tools as an aid. Computer software was used only to generate the final design outcome. The focus group in this preliminary stage was conducted with sixteen architecture students with various levels of design background. The common characteristic among them is a strong desire to design their own living space. 
Each participated as both an architect and a homebuyer. The duration was one semester (thirteen weeks), which might be insufficient time to conduct a fully detailed collaborative design process. However, as this is a focus group, the intensive process is enough to obtain sufficient data to analyse and understand the needs of the process. Another aim of the studio was to observe the struggle between flexibility and control, i.e., the conflict of the top-down versus bottom-up approach, and to investigate the potential problems faced with different parts of a building being designed separately yet simultaneously. With each student having his or her own ideas and goals, the design collaboration process was closely monitored. The sixteen students were separated into two groups of eight. They were both given an initial brief to understand and study the parameters of housing design that architects tend to use. Precedence was studied to understand various design components such as unit types, configurations, arrangements and circulation (Figure 40).

\section{MVRDV Silodam}
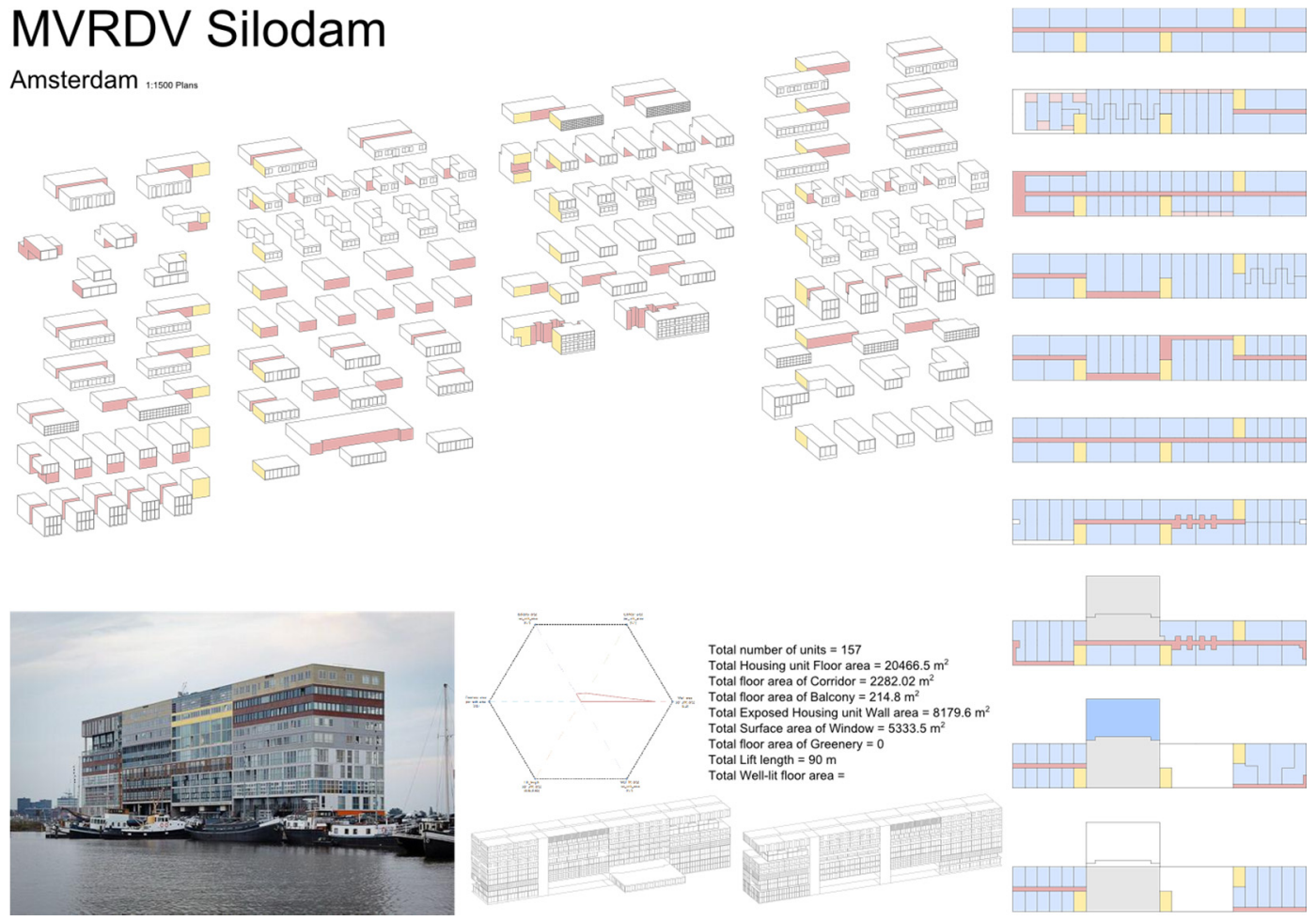


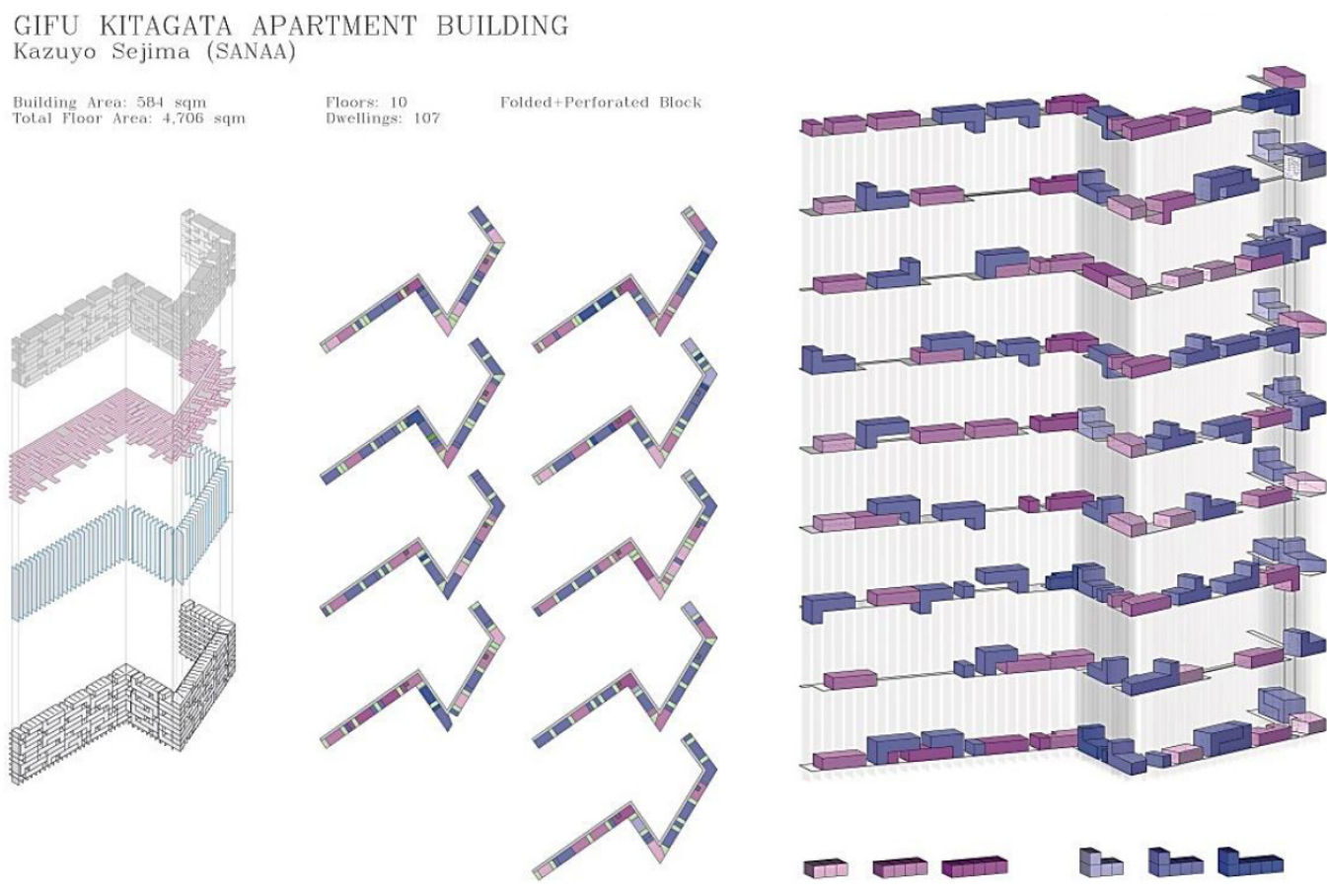

Figure 40 Precedent studies by some of the students

\subsubsection{DESIGN STUDIO SETUP}

For the actual design process of the studio, instead of the normal design workflow where participants are asked to design a building from scratch, this design studio is sequenced in four phases:

- Phase one - The design closely references the open building concept, in which housing design is divided into support and infill. Since the design studio's purpose is to observe the design collaboration process, this phase is the top-down segment where the support elements are prepared first. This also increases the amount of time available for interaction between students. The researcher conducting the studio set up the context and developed a building form in terms of layout and structure. In this case, a typical mix-use building with commercial at the bottom and residential at the top (Figure 41). A site context was established to determine how this might affect decision-making in the design process, adding another layer to the research discussion. The building is broken down into a grid of $3 \mathrm{~m}$ by $3 \mathrm{~m}$ with its core and structure specified. It is created digitally to provide a better understanding of the context for the students. The infill is then fragmented based on the plan grid for flexible selection and customization in the design process. 


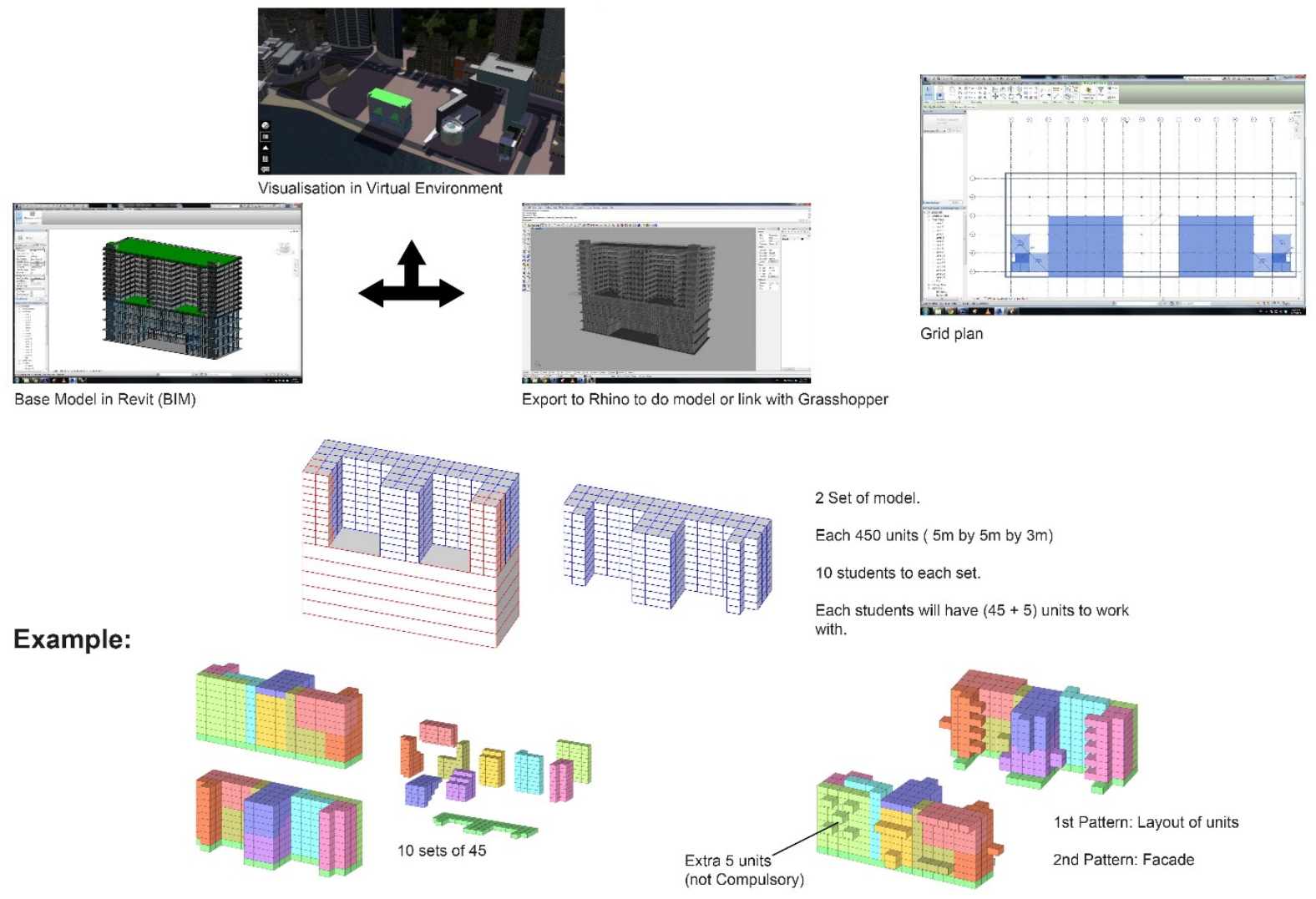

Figure 41 Setup of the design

- Phase two - This phase relied on the bottom-up approach, in which each student acted as a potential homebuyer with specific demands and needs, and tries to design to accommodate these as an architect would. This phase also examines how the level of design constraint impacts design decisions. The two groups of eight students were given different sets of parameters to follow during the design process (Figure 42). One group was provided with detailed design instructions while the other was given more freedom. Still, the design brief remains very raw at this stage to prevent the students from following rules and parameters blindly, yet not to the extent of allowing them to lose direction. The aim is to find the optimal point between flexibility and control. Each of the students then chooses their desired positions in the grid system. Within the chosen space, they arrange the grid units into habitable spaces that comprise any amount of grid units. Although they are each designing more than a single unit, this does not change the concept much. Each still has the ability to choose their neighbours. 


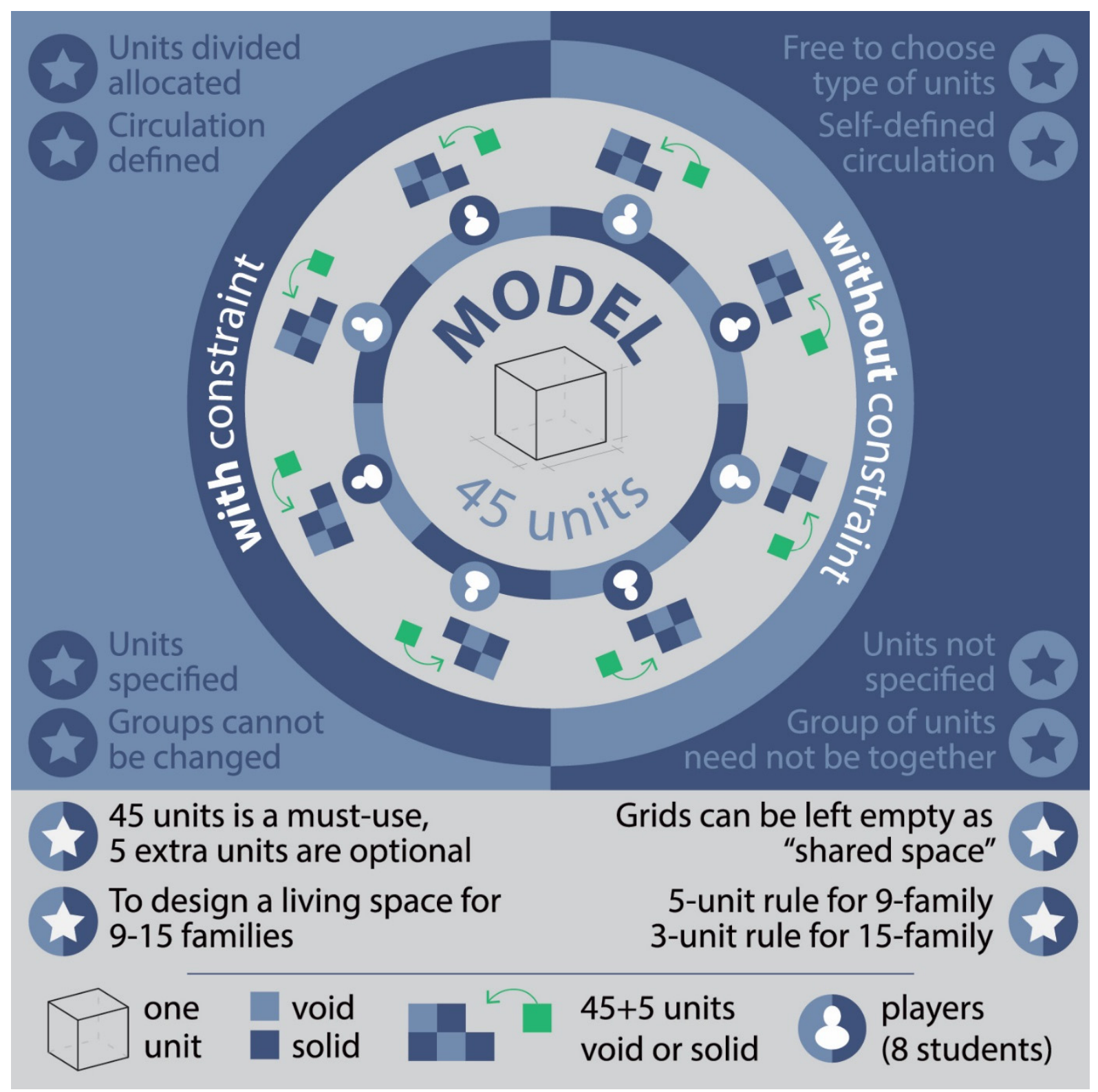

Figure 42 Constraints given to the students

- Phase three - With the grid units distributed and the unit typologies and floor plan defined (Figure 43), the students put their design together and analyse the outcome. Several rounds of discussion are then conducted between students to resolve any conflict of interest. Here, the researcher conducting the studio is a simple bystander observing communication and how collaboration is developed and conflicts resolved. Circulation of units and core, allocation of public spaces and greenery and the possibility of shared program spaces are considered and resolved among each group. 


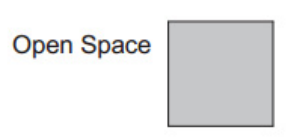

2 storeyed
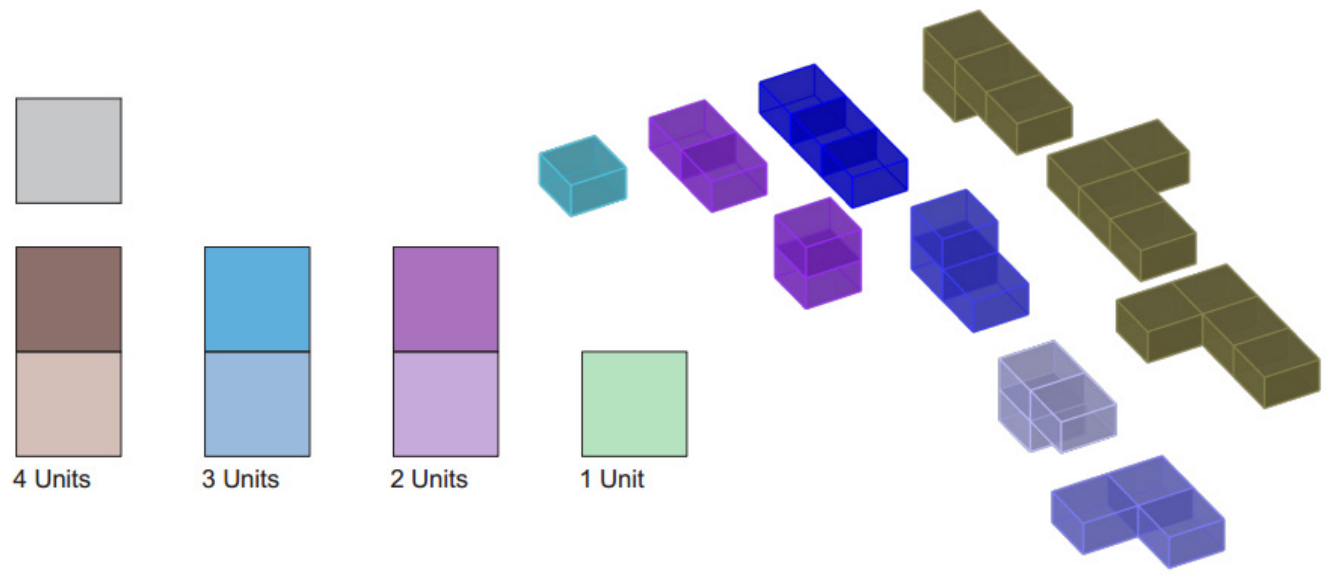

Figure 43 Housing types comprise of various number of units

- Phase four - At this stage, what is left is the façade of the building. Each designer has their desired direction of view, choice of opening types and even materials. The researcher steps in at this phase to maintain sufficient control to prevent the outlook of the building from becoming too chaotic. Privacy issues which could affect the unit layouts are observed at this stage and changes made if necessary.

\subsubsection{DESIGN STUDIO OUTCOME}

Under the condition of a fixed plan layout by the main architect, the students are each given a number of unit cubes to fill up the plan (Figure 44). Each cube does not necessarily resemble a housing unit; it can resemble a public space, a void or a green plot. The mission is to create an environment that each individual can imagine themselves living in.
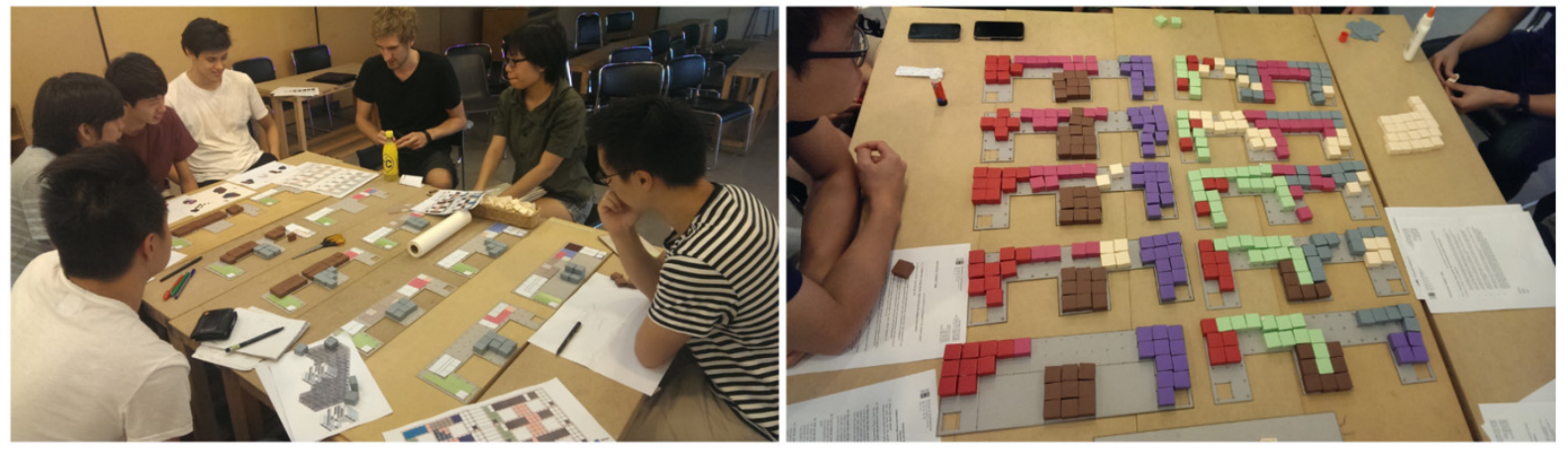

Figure 44 Images taken during phase 2 design process 
The students could change the constraints as they saw fit, provided a form of agreement is reached between the group and the researcher. Especially in the first group with more limitations, some constraints (such as those around the position of the lift core and structural components) had to be lifted to open up more possibilities and so allow better optimized design outcomes. Conversely, for the group without much constraint, new rules such as unit geometry were developed to ensure a certain 'style' consistency. The constraints in each group were then compared to determine the type of parameters the researcher could use to define the overall building level. This allowed the optimal control versus freedom to enhance individual creativity.

After laying out the units, the students worked together to generate a circulation that ensures each space is accessible. As this is just a design exercise, practical issues such as safety and fire escape issues are considered minimally; the only requirement is for circulation to connect to every housing unit and also reach the core. The researcher then collates the designs so the students can move to the next stage of planning (Figure 45). As they go into detail, potential spatial problems come to light that require the students to shift their cubes around; necessitating further discussion. The students meet weekly so most of the discussion and collaboration only happens then. The students work individually most other times and this caused many problems to arise; crashes of model components, inconsistent planning and issues of privacy where windows outlook to each other etc. At the same time, the students are packing units together so the researcher had to step in and request each group to provide more public spaces or increase the porosity of the building for more ventilation. 

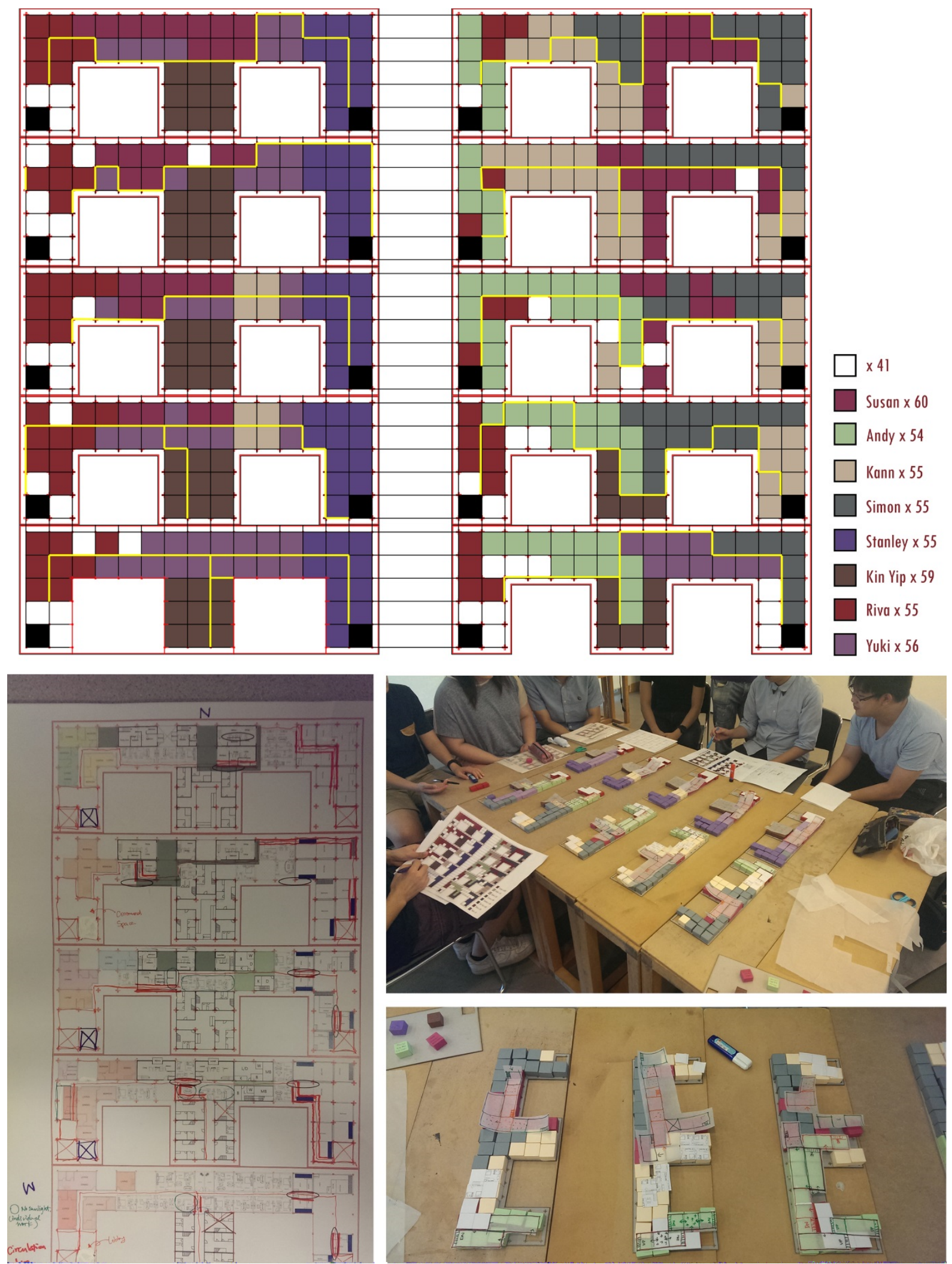

Figure 45 Various stages of design collaboration discussion, top image done digitally by researcher to keep track of the design progress in one of the focus group showing the circulation path decided by the students 

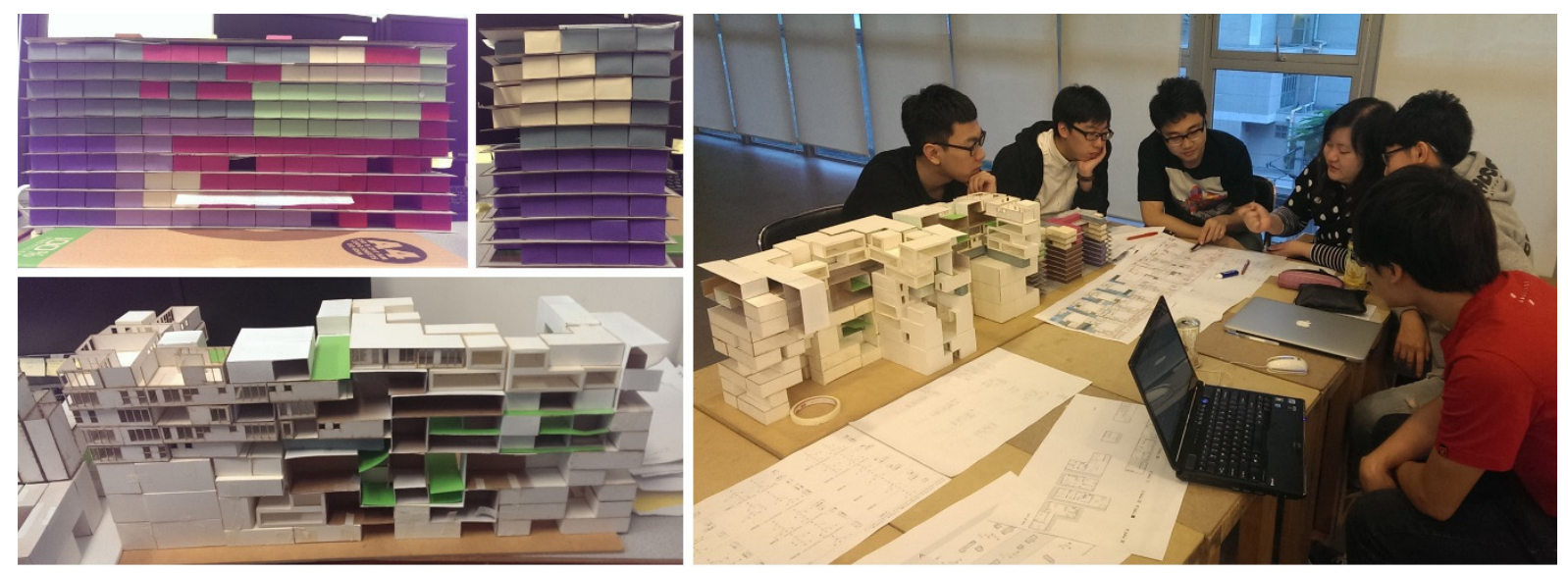

\section{Digital generation of outcome}

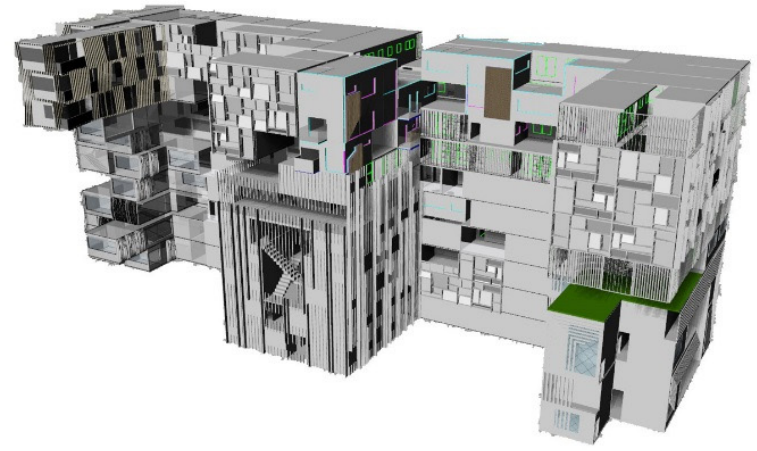

Group 1 Outcome

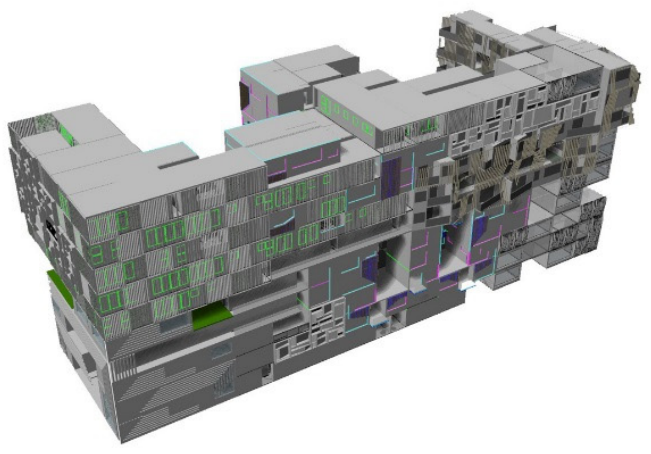

\section{Group 1 Outcome}

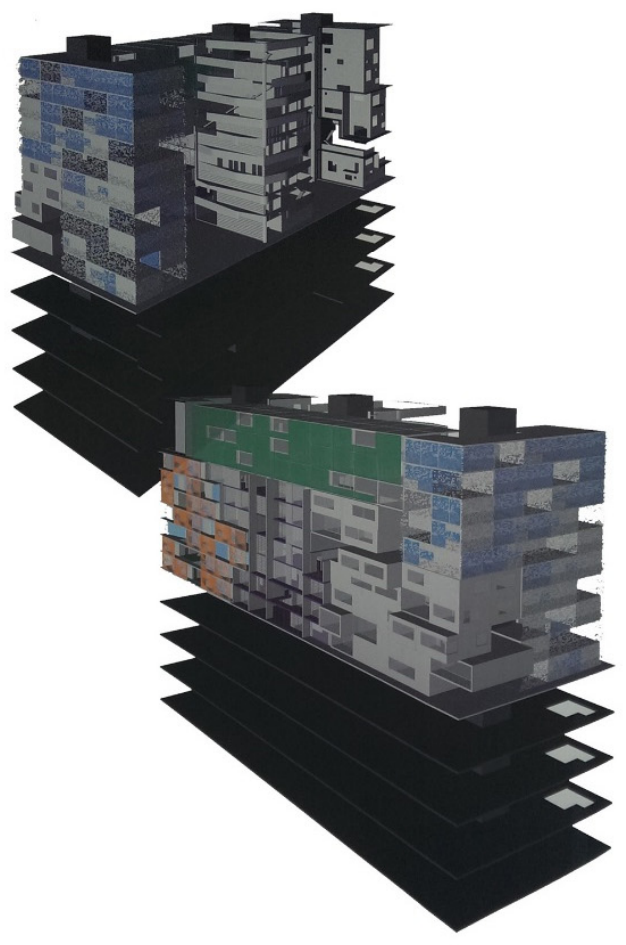

Group 2 Outcome

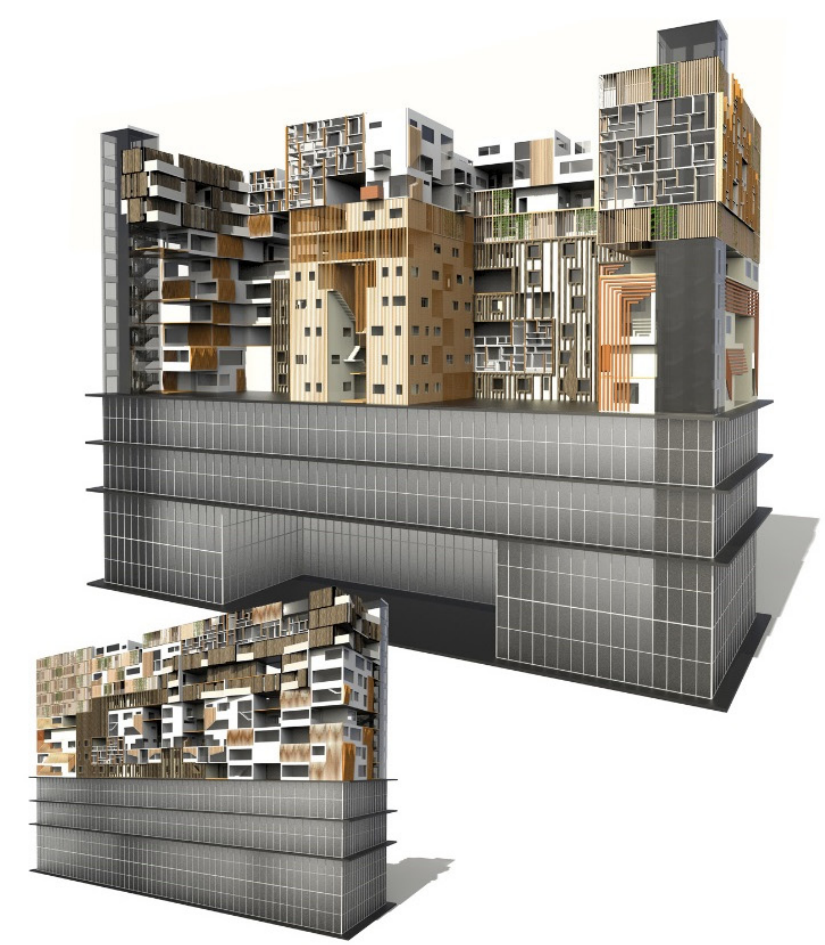

Figure 46 Further design collaboration discussion to work out the design details, progressing towards the building form and finally putting everything into digital to generate the final outcome 
After a few rounds of discussion and with agreement reached among group members for the unit layout, the students then proceed to phase four. The researcher collated the plans to check for any problems with the overall outcome. Rough physical models are built at this stage to give the students a better view of those parts of the building that are not receiving enough sunlight. This encourages another level of discussion, one which might need to revert to the planning stage (Figure 46).

In phase four, the researcher worked with the students to generate the overall façade of the building. In order to maintain the individual characteristics of each design, the method here takes reference from Frei Otto's Okohaus example in which the research gave only two parameters to each group: the general design concept of the façade is 'verticality', and the material is timber or wood. The students then design a façade based on these concepts. Collaboration still comes into play at this stage as they ensure all the designs come together coherently without losing individual design character.

\subsubsection{EVALUATION OF STUDIO PROCESS}

Observing the collaboration design process between the two focus groups, the second group with fewer design constraints showed more enthusiasm. They spent more time per session discussing the design possibilities and problems. They also formed smaller groups to distribute the problems that needed to be tackled. In contrast, all these collaborative elements were absent in the other groups. With the design parameters laid out, the members of the group were just following them and generating an outcome. Even though this second group faced similar design conflicts and issues, the collaboration and communication between the members was minimal. The contrast between the groups can also be observed from the level of detail generated in the individual outcomes. (Figure 47) 
Group 1

Student Work

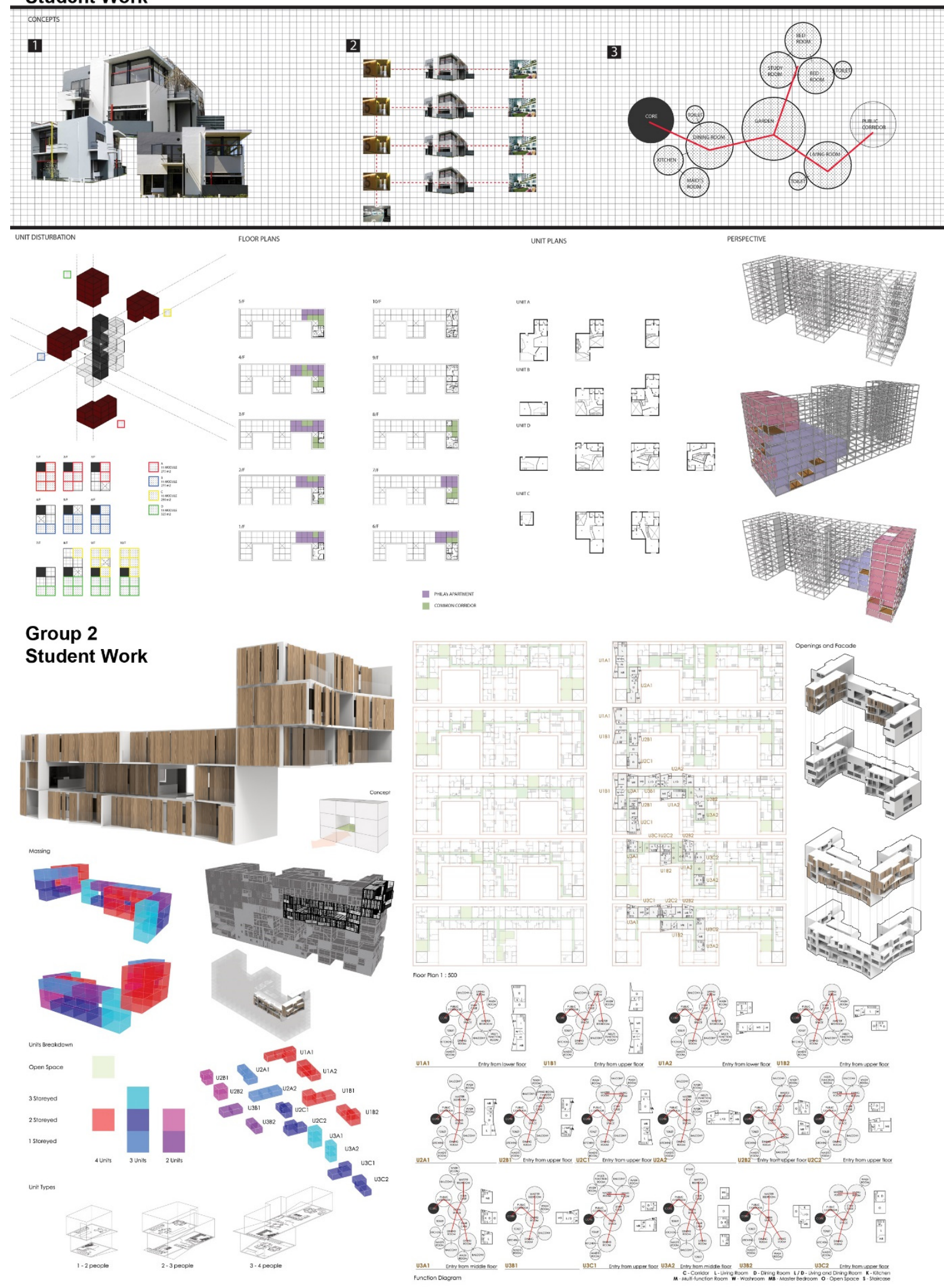

Figure 47 Detailed design of every units housing units by one of the students in each focus group 


\section{Feedbacks of Participants}

The two design outcomes were evaluated with respect to the constraints given. A design critique session was held where both groups presented their work to a group of professionals. They also took the opportunity to state the challenges they faced during the design collaboration process. One student commented that:

\section{"There are no guidelines as to how the units should come together. We are simply placing the units like a jigsaw puzzle."}

Both designs were then presented in the form of a public exhibition in the school compound. A few viewers were informally asked which design they preferred; most chose the design outcome of the second group. The sixteen students were also asked to answer a short survey about the desirability of the design method. All of them stated that a digitised process might be better. One of the students specifically stated that:

"It was really hard to get everyone together for the collaboration process, it would help if there is a system to bring everyone together or force them to come together but it is great to be able to design my own units."

By comparing the process and outcomes between constrained sets, this study finds that rather than giving specific parameters, it is more efficient for the researcher to provide general guidelines and limitations. However, this also requires the researcher to maintain a close inspection of every student so that they do not divert too far from the main objectives.

\section{Touching on Gamification}

The gamification techniques were subtly integrated into the process to test its capability. The breaking down of the building into grids simplified the design process for ease of collaboration. Using the eight core drives of gamification as a base, most elements used in this study are from the 'intrinsic tendency' category and the outcome of the design process indicates the response to using these core drives. By providing design freedom to one of the focus groups, the students are increasingly driven to generate an outcome based on desire 
and creativity. In brief, gamification elements can play a major role in creating the kind of engaging experience which is the aim of this project.

\section{The Next Step}

More importantly, this study demonstrates the demand for further research to create an integrated system to allow better communication among users and with the main architect, potentially promoting the formation of communities during the process. Data collecting is also necessary to facilitate information flow which, importantly, keeps every individual in the loop of each other's design progress and with the decisions made at every point in time. With such a large volume of information flowing, building information modelling (BIM) could also come into play. If the data is collated into a BIM system, it could improve design speed and efficiency. A web-based tool is preferred as this allows user-access anywhere and anytime.

\section{Possible Approach}

The design process can also adopt more of a community approach instead of purely an instruct-and-follow design approach. Each user can be responsible for a specific role such as circulation planning, public space function planning etc. As deduced from the observation of the design studio process, this method can improve collaboration between users in specific roles, allowing individuals an understanding of others' demands and needs before stating their own. There may be bias, but this is where the main architects step in. In other words, it is like designing a village but compressed into a vertical building. Everyone comes together to build their desired environment with the help of digital design tools. 


\subsection{The Design System: ModRule}

The information collected from the previous study was used to generate a digital platform ModRule. The name is short for 'modular rule' since housing units are usually modular in nature. With the rules and parameters set by architects and regional planning requirements, units can be varied yet retain modularity. Factors identified in the observation of the design studio were integrated into the development. Some objectives of this platform were that design parameters could be better controlled, communication could take place within the system, and that data collection could be managed more efficiently. The most important aspect was user-friendliness; the simpler the control, the easier it is for the homebuyers. Controlling the design content maintains the richness required to keep homebuyers engaged with the process. By setting flexible rules and parameters, the modular system is able to generate a diversity of design options for every individual homebuyer. The system setup closely follows that used in the design studio. As observed in the design processes of the studio, the open building concept of support and infill is insufficient. ModRule divides the housing design further into five major components: spatial driver, structure frame, skin modules, inner partition system and utility system (Figure 48). These components can still be categorised into support and infill but these subdivisions allow for more design flexibility and for the architects to have control of every aspect of the design. Using a BIM methodology, components are not separated but rather linked together to correlate and maintain information throughout the process. The five components offer the possibility of selectively controlling information; information which can be published as open source for others to use or contribute to.

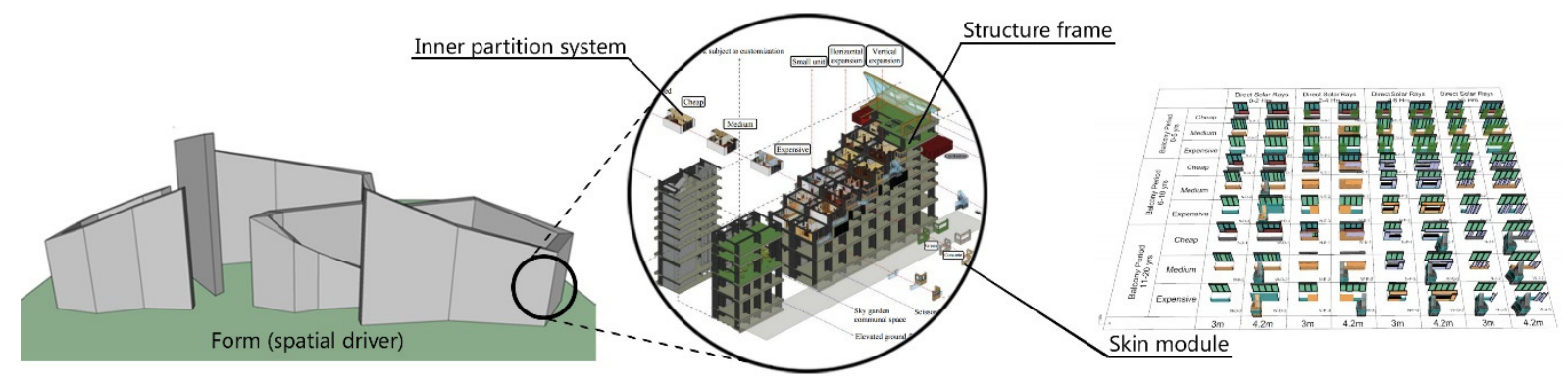

Figure 48 Five major components of mass housing design: spatial driver, structure frame, skin modules, inner partition system and utility system 
Within these five components, there are countless possibilities for participants to develop meaningful design outcomes. Two of these components, skin modules and inner partition system, allow sufficient flexibility for design solutions that respond to the design parameters set by the architects. To open up these two components for homebuyers' participation, the architects must design the other three components specifically to accommodate design variations. In other words, the architects have two critical operations: One, to construct the three components which include creating the basic building form, developing the structure frame accordingly, and setting the public space and utilities. And two, to establish the variation schema of the other two components. This includes laying out a range of skin modules and working out the arrangement of the inner partition system, allowing types of input parameters such as daylight, sky-view factor, accessibility, thermal radiation, cost, etc. In this situation a BIM model is necessary to manage information and connect the data of the dynamic open source model to the construction documents, without consuming extra resources. The challenge for the architect is providing a BIM model that can respond to the wide spectrum of data that results from the various design options generated by the homebuyers. 


\subsubsection{INTERFACES OF MODRULE}

With the design workflow structured properly, a first prototype is developed. It is divided into two main groups, i.e., the administrator for the architects and the public for the homebuyers, but has four parts to the interface:

- "Create" interface (Figure 49) - this is the administrator interface where the architects prepare their framework of the design by setting the initial design rules and parameters. The basic design of the mass housing building is divided into grids of various sizes depending on the architects' preferences, and every grid space can be assigned with parameters and variables. The architect can grid the plans with respect to his or her plan geometry, i.e., it need not be a square grid if the architect is designing a unique housing plan. The grids have parameters attached to them such as space requirements, budget, orientation, daylight preference, etc. quantified and predefined by the architects. The building plan is laid out according to necessary elements such as building core, access and exit routes, utilities, etc.; these grids are set as "fixed", and homebuyers cannot select them. In principle, the predefined grids in ModRule can be designed in any manner the architects require. Consider a Japanese tatami mat which is has a standard dimension. The layout of Japanese houses and apartments are based on the dimensions of one tatami, which acts a base unit of space. The amount of variation can be controlled by the architects to keep the design economically viable and efficient. With these factors defined and set up, the design is ready for homebuyers to collaboratively develop and generate the design of the mass housing.

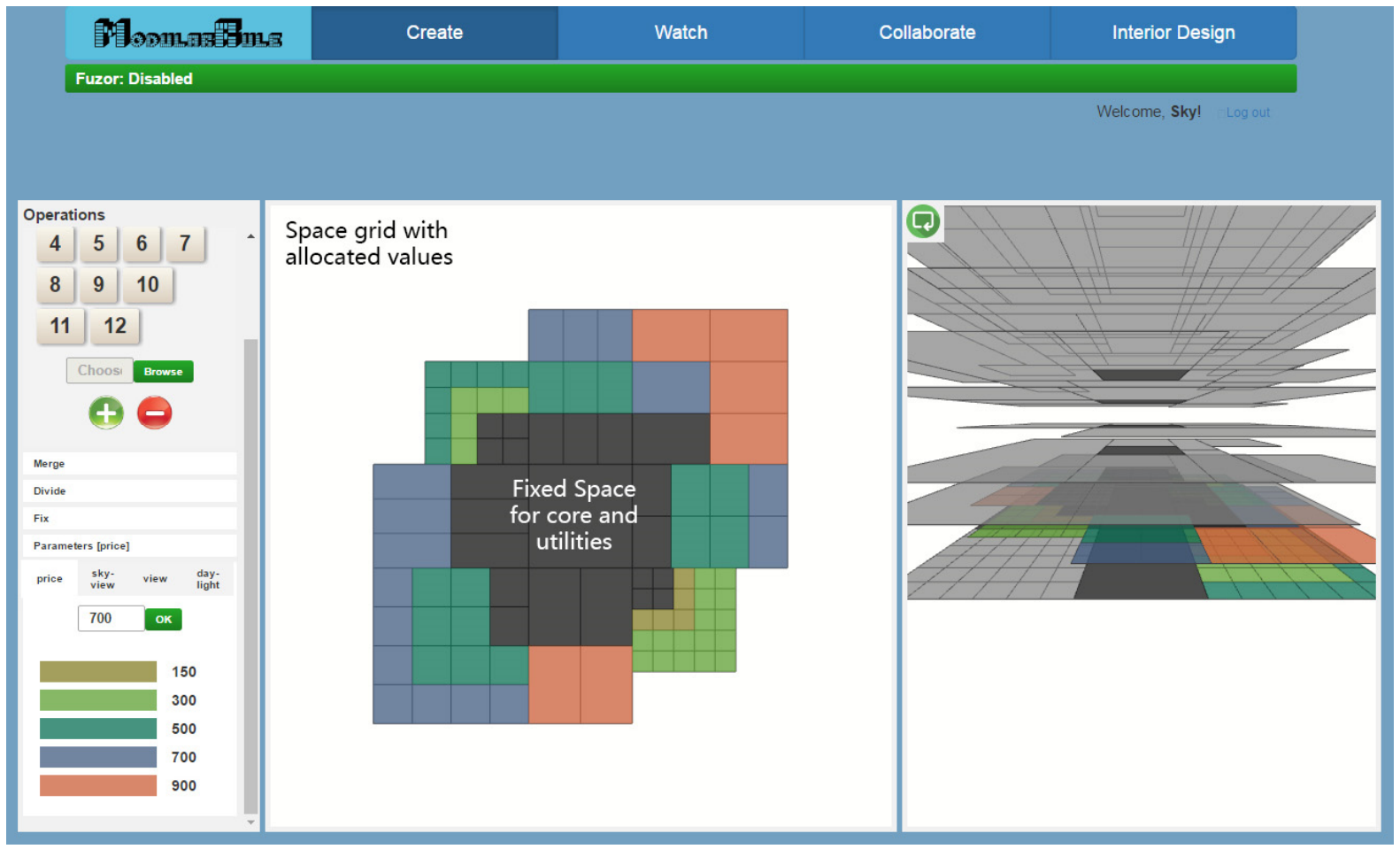

Figure 49 "Create" Interface of ModRule system showing the fixed building core and parameters (coloured) set by the architect 
- "Collaborate" interface (Figure 50) -homebuyers interact with other homebuyers collaboratively in this part of the system to generate a layout featuring their preferred space and characteristics. In this interface, homebuyers see only the plans of the building. The building information and parameters added by the architects, such as orientation, available view types, and amount of sunlight are indicated in the diagram as part of the model. Through the interface, the homebuyers first set their targets or identify factors that contribute to their desired home design. The design collaboration with homebuyers starts with setting a goal system based on their personal profile. This goal system is like a checklist of targets that homebuyers follow. It references the 'objectives' in games that players try to achieve while playing. A 'goal bar' is available in the ModRule interface that interacts with the parameters and variables input of each grid. The goal bars fill up accordingly while choices are being made by the homebuyers, indicating whether they are achieving their declared end. The role of the goal bar is to enable every individual to manage their desires. This ensures that homebuyers are not designing a living space that is bigger, or more highly resourced, than that specified in their user profiles. The information of every homebuyers' goal bar is available to all participants, encouraging sharing, understanding, and social interaction. Ideally, this transparency will encourage every homebuyer to help others to achieve both their personal and their common living spaces as all homebuyers will ultimately live together in this building they are jointly developing.

Depending on the project it can be almost impossible to fulfil all personal goals because of the need to compromise with other homebuyers. Participants can certainly aim to achieve as many as possible. There might be occasions where goals are compromised to achieve a solution that fits common interests. These situations are to be negotiated by the homebuyers themselves, offering every participant a better understanding of the overall process and potentially a higher acceptance of the outcome.

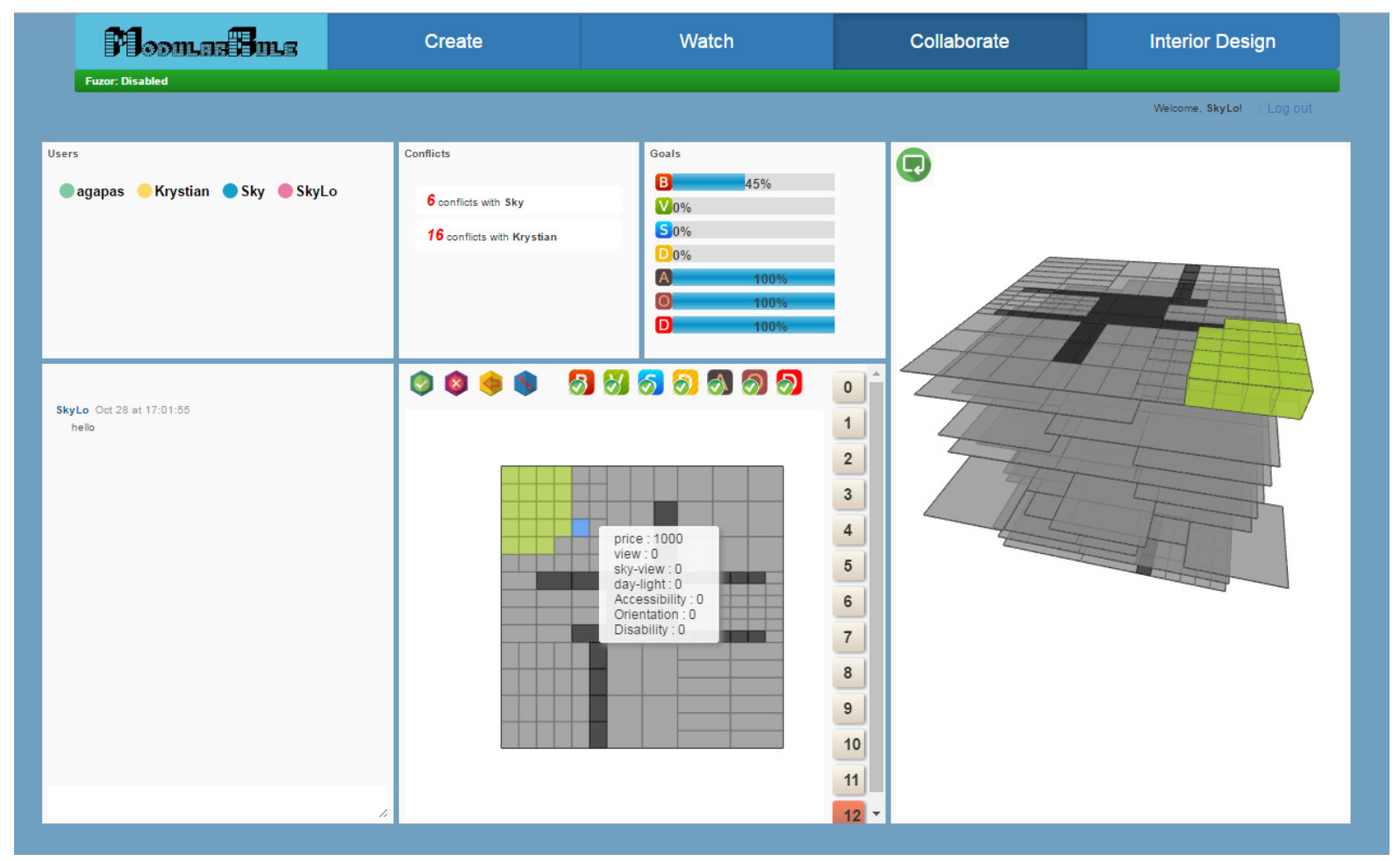

Figure 50 "Collaborate" Interface of ModRule system showing the what the homebuyers see while interacting with the interface and collaborating with other homebuyers 
- "Watch" interface (Figure 51) - here, the architects communicate with all the homebuyers and oversee the whole collaboration process with data gathered from each individual's movements. The interface is similar to the "Collaborate" interface. Every conflict is visible, and the architect can consider the targets of each homebuyer to provide the best win-win situation for everyone involved. For example, the homebuyer with "view" as their first target will prioritise units with a high view value, unlike another homebuyer with "view" as their second target. However, if the former homebuyer has already achieved $80 \%$ or more of their target, the latter can be given priority to meet theirs.

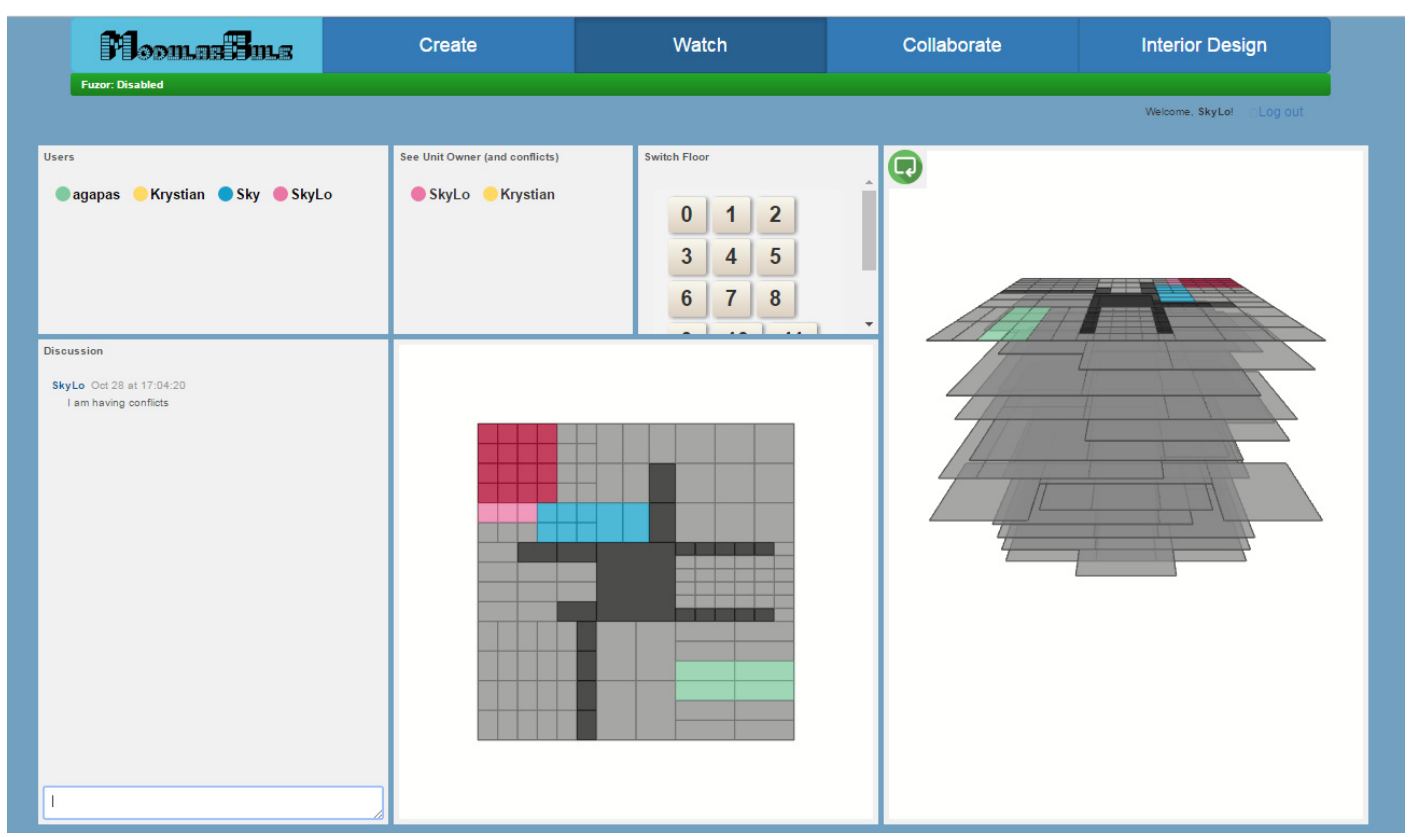

Figure 51 "Watch" Interface of ModRule system showing the choices of the various homebuyers and the conflicts in red

Meanwhile, as the architects oversee the process they define the skin module and inner partitions (Figure 52). The amount of variation can be controlled by the architects to keep the design economically viable and efficient.

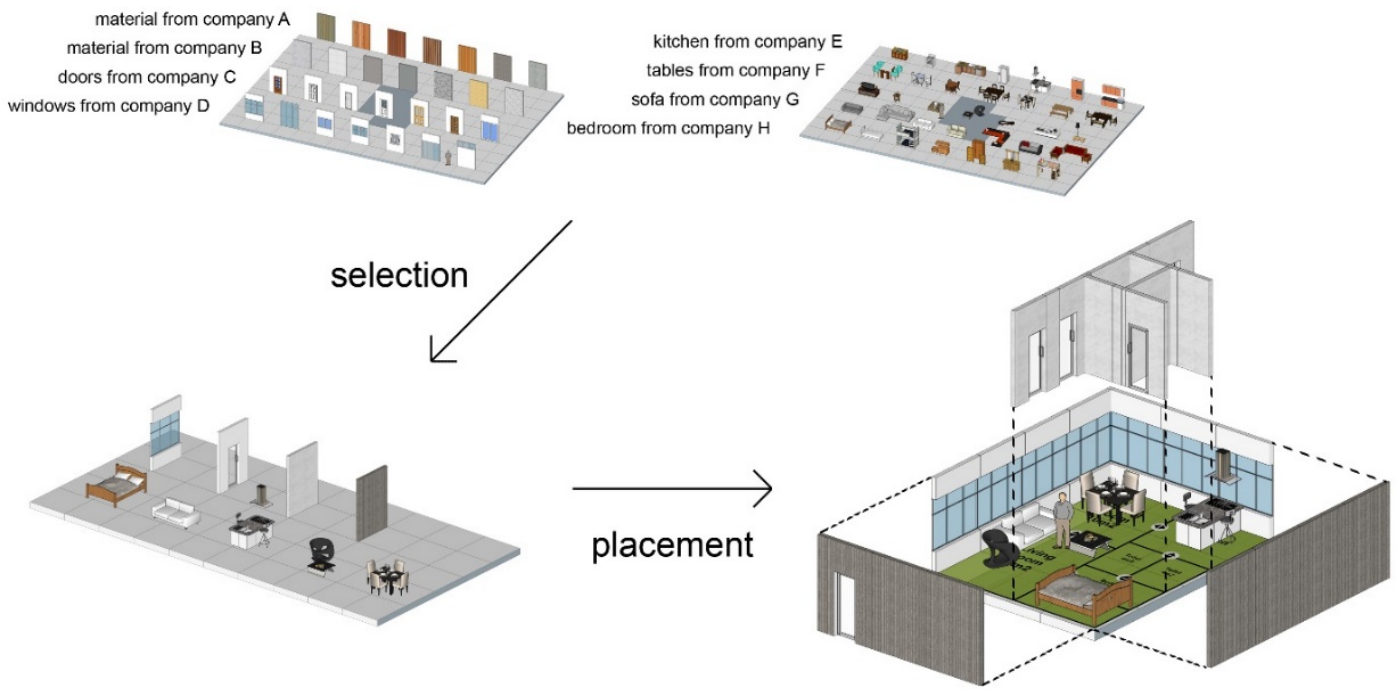

Figure 52 Defining the set of skin module and inner partitions for the homebuyers to select and place them into the space they created 
Once everyone's goals are achieved to a minimum threshold (i.e. above $80 \%$ ) and to a point of consensus on the outcome, the design stage starts. This is where the individual living spaces are synthesised to generate a common overall architectural design. The 'ingredients' prepared by the architects are applied as homebuyers use these and architectural components to select and design their space. At this stage, architects take the leading role. Although the aim is to achieve an overall design through a bottom-up, 'democratic' process, top-down aspects are not negligible especially in this context of mass housing. There are too many building and architectural issues requiring the knowledge of professionals.

- "Interior" interface (Figure 53) - homebuyers interact individually with the model to 'design' the interior of their desired living space. They do not design plans from scratch. Instead, homebuyers select the spaces they want to have and choose the space they want to connect it to. The interface then generates the overall spatial connections in real-time for the homebuyers to view. Once satisfied with the arrangement, homebuyers can click 'submit' for the system to generate a floor plan that fits the proposed arrangement. Since one spatial arrangement does not necessary mean one floor plan outcome, homebuyers can resubmit the same arrangement to regenerate floor plans until they see the best fit. There is also the option to go back to rearrange the connections.

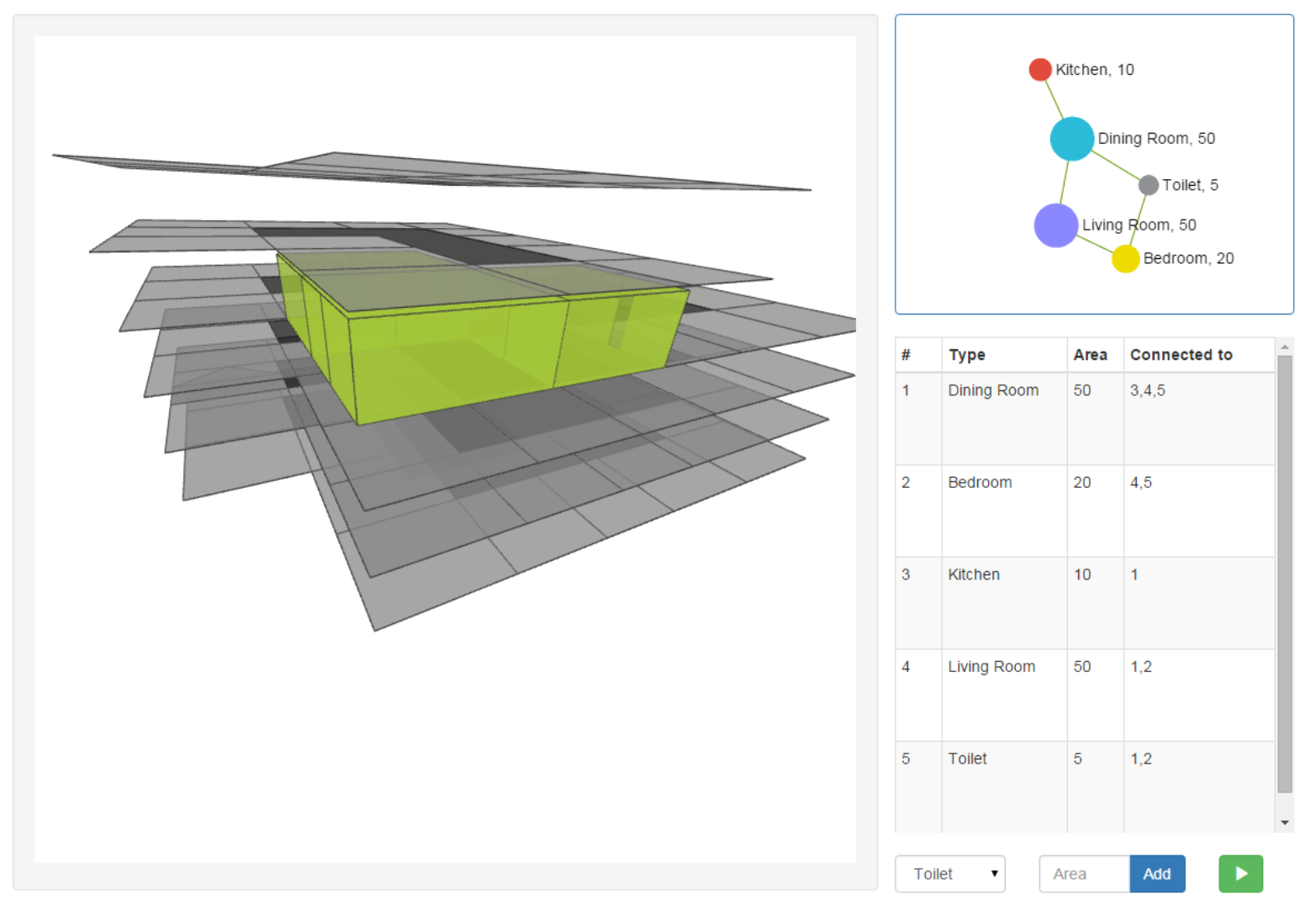

Figure 53 "Interior" Interface of ModRule system showing the spatial connection of the various functions that one homebuyer hope to have 
In summary, the design process workflow using ModRule can be described as in the figure below (Figure 54). The figure demonstrates how the system can work for collaboration between homebuyers, and how the information it generates can work for other stakeholders too:

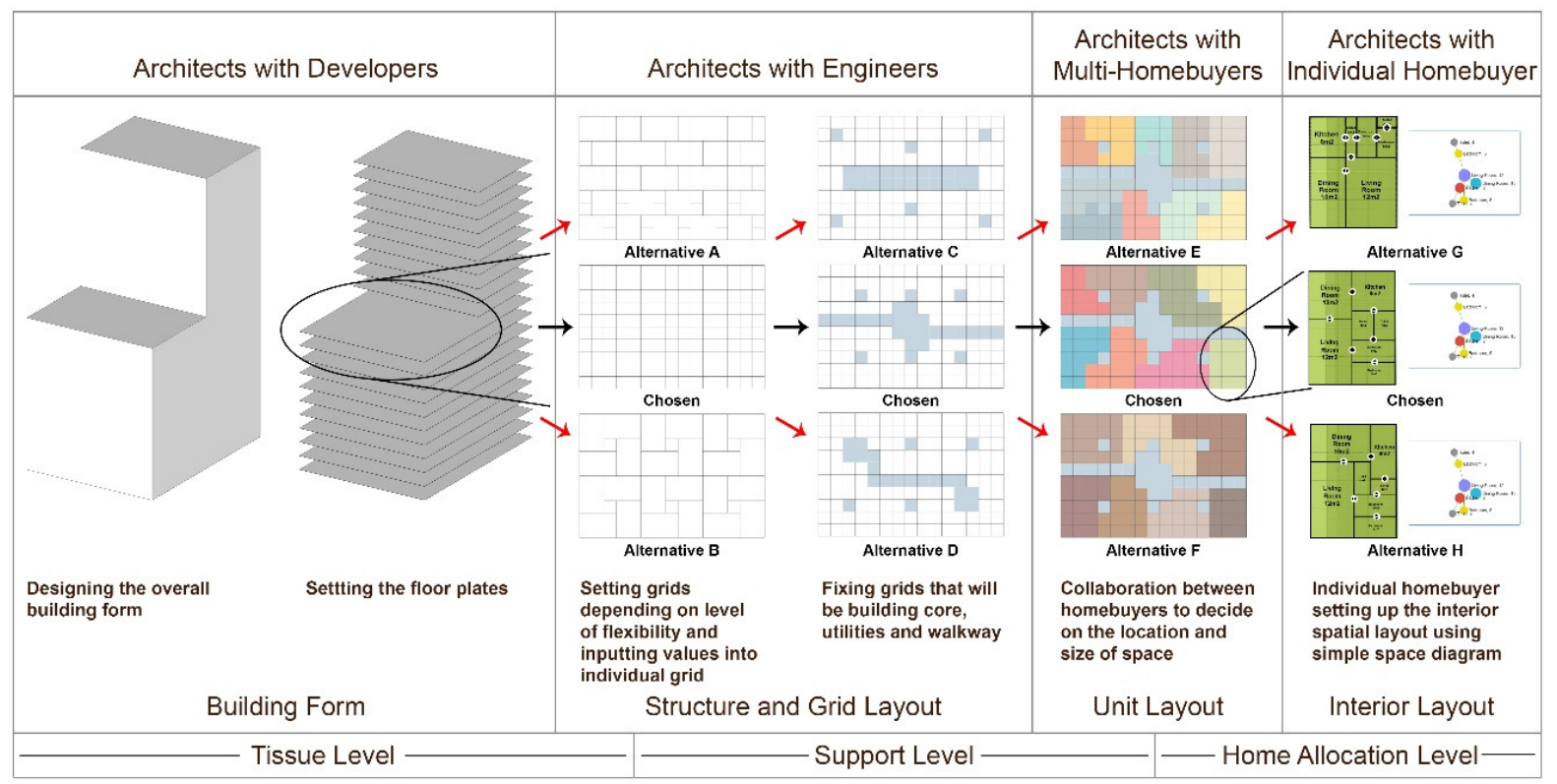

Figure 54 The design collaboration workflow with ModRule

\subsubsection{SYSTEM STRUCTURE OF MODRULE}

The technical aspects and functions of ModRule are supported by setting rules and parameters crucial to end users. This is inspired by simulation game designs to provide an 'easy-to-learn' design process for the bottom-up collaborative approach. Using Java-Script-based code, WebGL, the proposed design tool can generate a wide variety of design options for individual occupants as well as negotiate conflicting interests and outcomes. The system can be simplified into three main modules: the 3D design module, the data management module and the real-time communication module (Figure 55). 


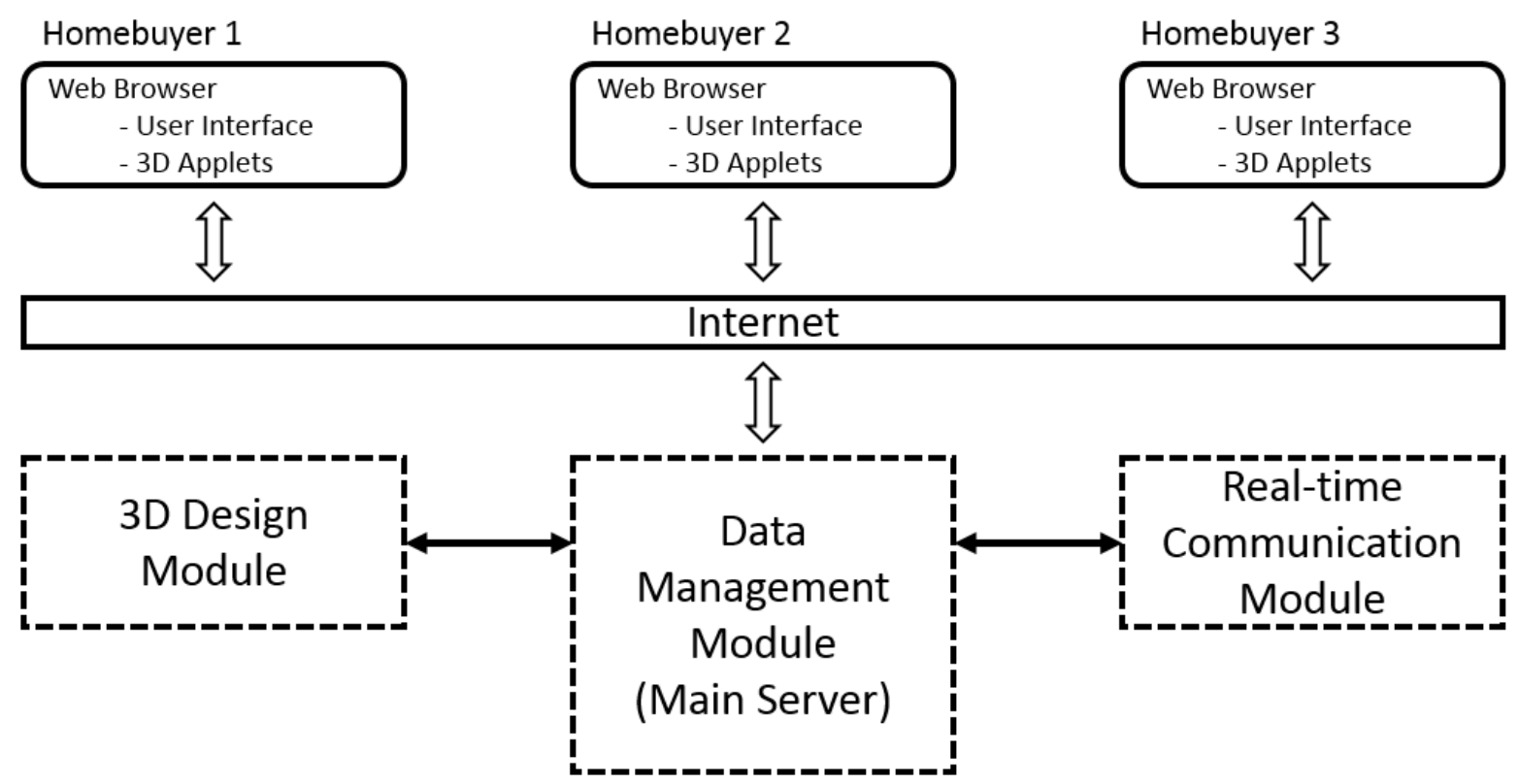

Figure 55 The system structure of ModRule

The 3D design module first takes STL files as input and converts these into a triangulated geometric model. The model is then digitally manipulated by architects using an open structure that allows them to set design constraints and the level of design freedom. Physical and environmental parameters are mapped into the geometry accordingly, which then act as a 'scoring' system for users during the design process. The data includes user profiles and are stored in a data management module that uses 'redis', an open source, in-memory data structure store. For the communication module, Node.js is another open source code capable of providing scalable network. This system, therefore, utilizes Node.js to facilitate realtime design communication. Simple text-chatting remains the best form of physical communication, so this is implemented in the interface. Incorporating the three modules using WebGL provides the ideal solution to generate $3 \mathrm{D}$ geometry in a web-based interface for collaborative processes through the web and helps to increase the speed of communication essential for decision-making during the design process.

\subsubsection{TESTING OUT MODRULE - THE PARTICIPANTS}

A study of the tool was conducted to ascertain if ModRule can achieve the aim of this research; to act as the medium for design collaboration. In this study, a second focus group was recruited 
to work with ModRule. This included nine architecture students with varied backgrounds who volunteered to participate in this study. Like the previous study, these students have a common interest in, and knowledge of, housing design. The setup of the design process is also similar. The difference is that this study substantially engaged the digital tool and participants worked individually, rather than in groups; each responsible for the setup of their own parameters and rules. Moreover, no constraint was predetermined in this study of ModRule, so participants had more design freedom. The design process changed significantly with the need to set up a design framework instead of designing a full building. This requires a certain level of understanding of rule-setting and parametric design thinking so participating students were introduced to a series of programs to prepare them for the design process.

\subsubsection{TESTING OUT MODRULE - THE PROCESS}

The first program introduced to the students is a game titled 'Prison Architects'. It is developed by Introversion Software for people to design a functional prison. This game is like 'Sim City', a construction and management simulation type of video game, just that players are not designing a city, but simply a private prison. Although it is a game, the rules set, the parameters, the design components, and even the spatial relations are set up comprehensively for players to design and operate their own prison. This game relates to BIM in a compelling manner. For example, by telling the system what space they have created, the players can only include certain furniture and require a specific number of utilities, such as water and electricity. Although as complex as architectural design, the way that the game provides guidance and requirements for each spatial type enables the players to design the prison without much difficulty. However, as simple as it may be, players must play several times to truly grasp the whole gameplay. This is very similar to housing design, in that the players benefit from experimenting; by 'playing around' and exploring as much as possible how to configure their living space and determine which one best suits their needs and works out practically. 'Prison Architects' is, however, still a game, and the result is just a two-dimensional plan with agents moving around. 
Secondly, the students were introduced to 'Starlogo TNG', a system developed by a research group at MIT to teach programming to children. The interface is designed to be intuitive, yet the interaction with the system is very rich. The objective of this exercise is to allow the students to have some hands-on experience with setting up rules. To gain more established skills for programming, coding in 'Processing' Programming Language was also taught.

The design process is very different to the previous study so more time was spent to allow the students to understand the concept of rule-based design. As the students still played both roles of architect and homebuyer, technical knowledge is necessary for them to fulfil the role of the architects. With the ModRule system, however, playing the role of homebuyer was expected to be easier with less time required for the design collaboration process. The process began with every student playing as architects to design an overall building design framework in ModRule (Figure 56). Having set their design framework and parameters, discussion and even trial play was done with each design to rate its advantages and disadvantages in terms of flexibility and constraints of the set parameters. Then, of the nine projects, the nine students voted for the one with the most interactive setup (except their own). The selected student then continued to act as the architect while the rest converted to homebuyers and tried to achieve their desired living space using the selected framework. 

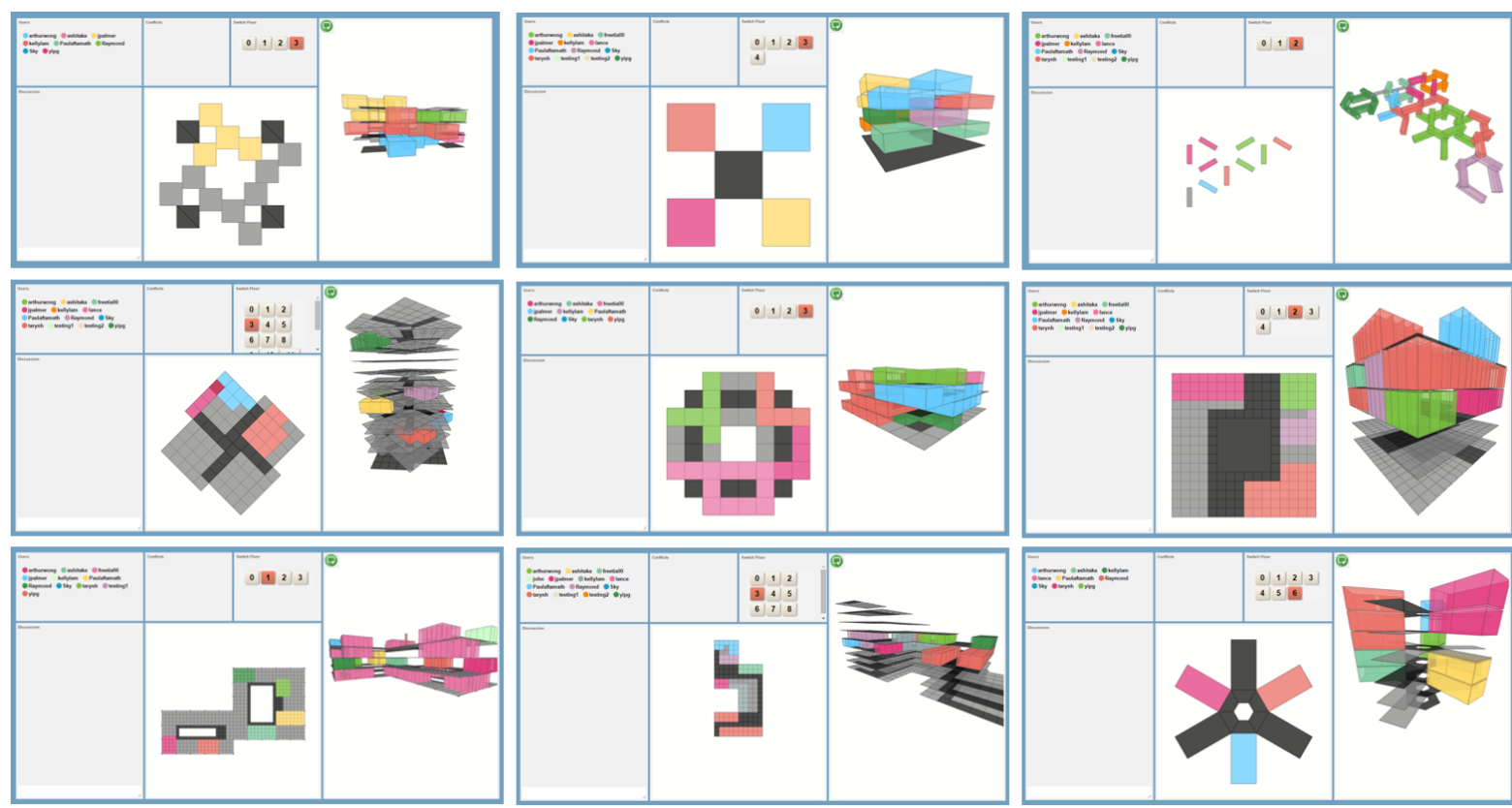

Figure 56 The design form of each of the nine students

\subsubsection{RESULTS FROM MODRULE}

At this stage, ModRule was still a prototype, and therefore some design setup had to be established manually. This included having each student build a general massing form, and write a detailed plan to prepare for the 'gameplay'. The rules were quite general so that the gameplay could be more flexible. As the design progresses, the rules might change to allow the design to converge to a more specific outcome. With the experience gained in the previous exercises with various games and applications, the students even designed the characteristics of the homebuyers (Figure 57) so that the gameplay could be more interactive and simulated. Each of the eight remaining students can play a specific role and try to achieve a desired living space for their character. For example, if a student plays the role of a family of four, he or she should not be located beside a single family which dislikes children; an elderly family is preferably located at the lower level and closer to the loft core to have better access to the ground level, etc. For every character to achieve their desired living environment, several rounds of discussion and negotiation had to be performed. Compared to the previous study, the availability of a digital platform enabled the design to be changed and adjusted easily. The 
parameters enhanced the negotiation with a clearer understanding of each other's needs and criteria.
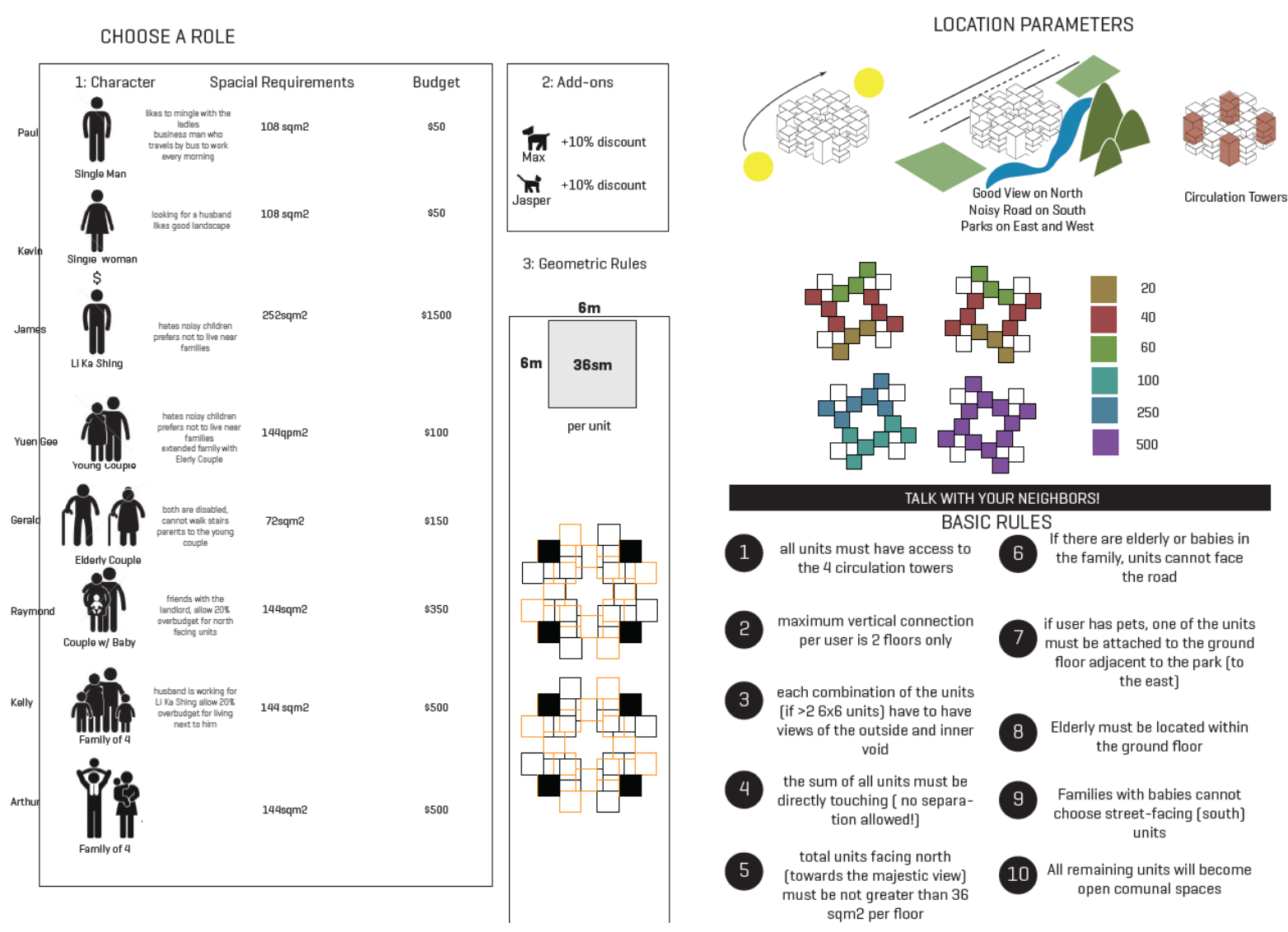

Figure 57 Rules and parameters set by one of the designers

The open system worked for students to collaborate on inventing potential designs. This research also seeks to understand how architects might design using the proposed method. An interesting initial observation was that one student over-set the rules, resulting in little to no freedom for the other students to choose their desired spot in the building design. Not only did this student set the parameters, the unit types were also specified for every role she created to the extent that the other students were just finding "correct" spots in which to place their units (Figure 58). The outcome was very limited, and the other students experienced difficulty most of the time. The result was very similar to what the student had planned. It was as if she designed the parameters so that the outcome would end up what she desired instead of what the other students might want. 
RULE 2

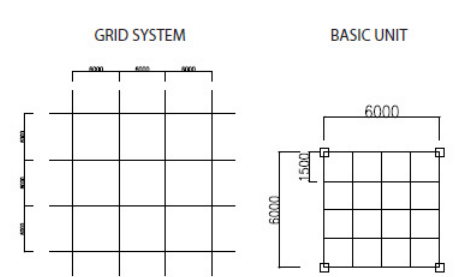

Grid System \& Basic Unit Rule

1. It is free to choose any single basic unit.

2. choose more grid base on the choosen basic unit and different unit type from RULE 1.

4. all unit should get unblocked view.

5. empty space between picked units will be a garden.
UNIT TYPES

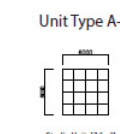

Unit Type B-1

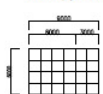

Unit Type C-1

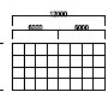

3.-bedionum Unit [T2m!

Unit Type D-1

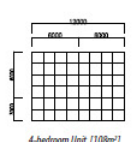

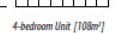

Unit Type E-1

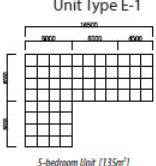

SUB - TYPES
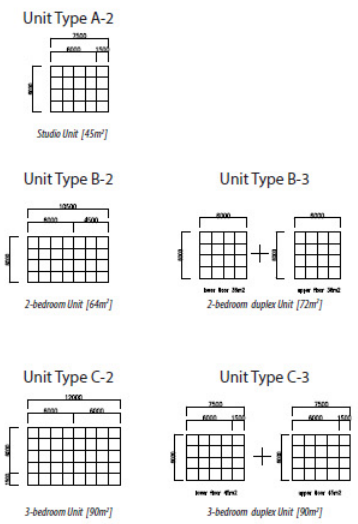

Unit Type D-3
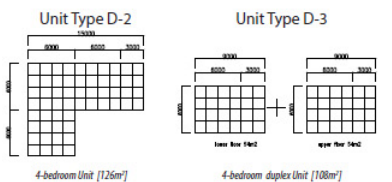

Unit Type E-2

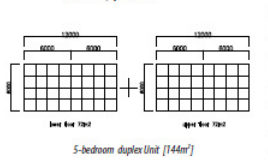

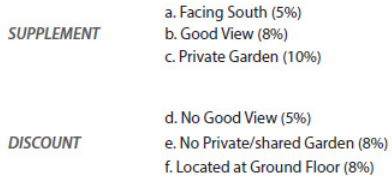

d. No Good View (5\%)

$\begin{array}{ll}\text { DISCOUNT } & \text { e. No Private/shared Garden (8\%) } \\ \text { f. Located at Ground Floor (8\%) }\end{array}$

PLANS FOR REFERENCE
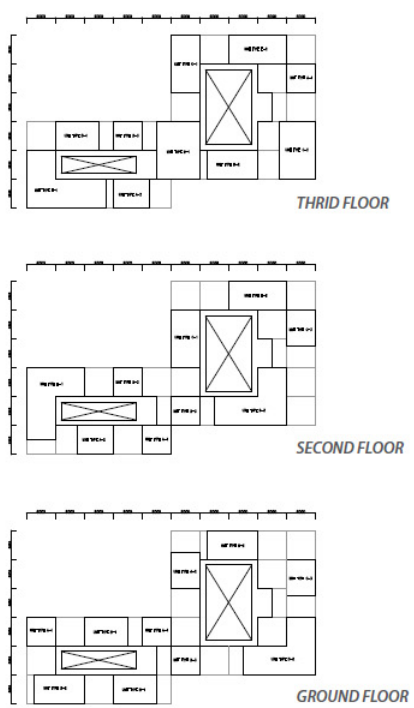

Figure 58 Rules and parameters set by another designers

\subsubsection{EVALUATION OF MODRULE}

The selected project went through the design collaboration process in ModRule and when the objectives of the created homebuyers were fulfilled by the students, they moved on to the next stage of fully designing the units. Detailed plans were generated from the abstract units to define the function of the houses. The success of the outcome (Figure 59) was easily evaluated, with the goal bar showing most of the 'homebuyers' achieving around $75 \%$ of their objectives. 


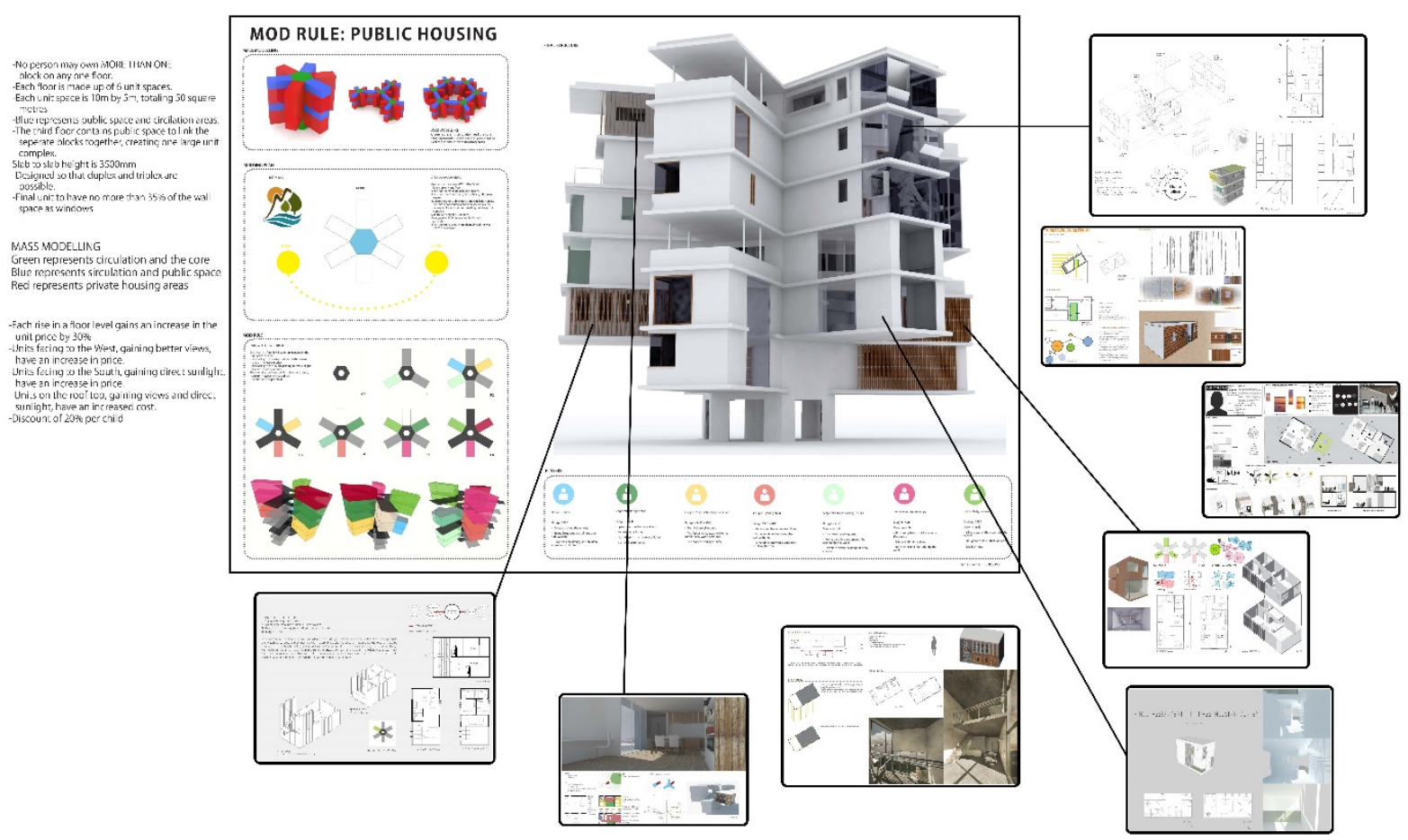

Figure 59 Final outcome of the chosen design

\section{Feedbacks of Participants}

When compared to the previous preliminary study, the students here showed a better understanding of rule-based collaborative design. Exposure to various games and programs before introducing ModRule gave them a better understanding of the purpose and flow of ModRule. During the planning of their rule sets and parameters, the students knew what to look for to ensure smooth collaboration and a design language to be communicated. When asked about the design system, one of the students commented that:

"The learning process is fun, but the design process can be automated more as it is still too tedious right now"

The previous group (design studio) took longer to reach the stage of generating rules, and the rules generated caused quite a lot of conflict. This required a substantial amount of time to have the design adjusted and refined. For the current group (ModRule test), however, although the students played only the role of the homebuyers most of the time, the study still provided a better understanding of ModRule. The fact that fewer conflicts appeared and the 
students attained their targets and aims more easily showed that ModRule is capable of handling collaborative design. The only setback was not with the system but the design process as the participants, while playing the role of the architects, needed a different design thinking (computationally) to be able to set up the model parametrically to allow this collaboration to work. One of the students who faced the most difficulties stated that:

\section{"Setting rules and parameters are hard, it is so different to how we normally design. And it is very hard to visualise the design in the current system"}

However, a short post-design interview conducted with the focus group students proved that with the help of a digital platform, they had an easier and more effective way to engage with users.

\section{Touching on Gamification}

With respect to integrating gamification, ModRule tries to incorporate the elements learned in Chapter 4 and test its capacity in this experiment. Further analysis is explicated in the next chapter, but at this stage it is certain that gamification does play a significant role in enhancing the design collaboration between participants. When asked how ModRule compared to the game 'Prison Architect' and 'Starlogo TNG', one of the students commented that:

"This system [ModRule] does not feels like a game at all, the design process is ok but the interface can be much better for us to interact with the design"

\section{The Next Step}

One very important element was identified while observing this test of ModRule: Virtualization-and-Visualization. ModRule is currently a simple web platform and substantially lacking in visual capability in the context of the design set-up. The current studies were done by students imagining the site condition. The availability of views, the amount of daylight, and orientation were all drawn on paper and referred to during the collaboration process. Students participating in the research studio also displayed difficulties imagining the context. 


\subsection{Bringing ModRule to the Public}

To be able to answer the research question more directly, visual enhancement is necessary. The aim is to provide a much more visually clear model so that homebuyers can understand the design and objective intuitively, instead of requiring a detailed explanation of the models. Homebuyers are unlikely to be architecturally trained, and the current abstract digital building form displayed in ModRule can be difficult for them to understand. A visualization of site environment and conditions will aid the public to set their desired living conditions more clearly, and hence enable the research to be conducted with much more fruitful results. It is a huge challenge to enhance the capability of ModRule by incorporating a high quality rendered environment into a web platform. Instead, third-party software is adopted to fuse ModRule with a visually advanced interface that relates very closely to BIM. Fuzor, an interactive and realtime virtual reality visualization and evaluation plugin for Revit ${ }^{\mathrm{TM}}$ developed by Kalloc (2014), provides the necessary capacity to enhance ModRule and so provide a more engaging interface for homebuyers.

\subsubsection{MODRULE WITH FUZOR}

Connecting with Fuzor is simple. Using a Fuzor-developed API (Figure 60), external applications such as ModRule can exploit the functions in Fuzor. Since the aim of Fuzor is to act as a BIM solution for the AEC (Architecture, Engineering, and Construction) industry, and since ModRule needs BIM to make the design collaboration more practical, this provides an opportunity to connect with Fuzor and enhance ModRule.

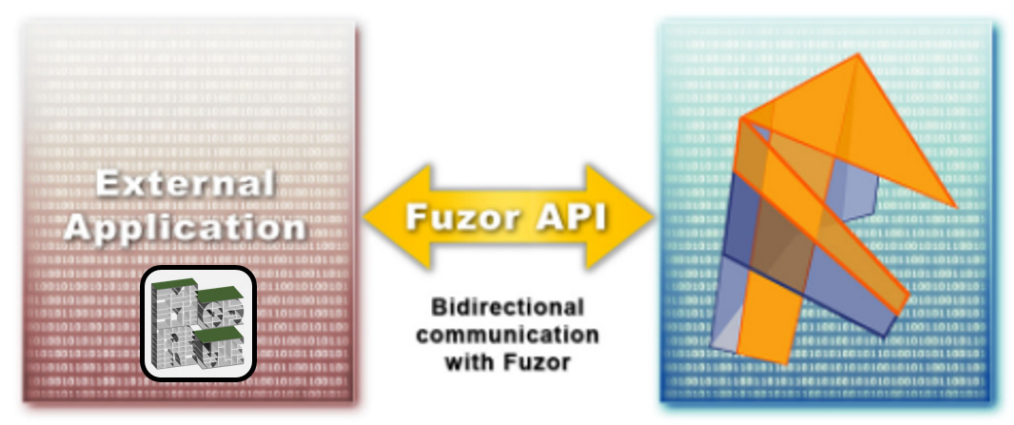

Figure 60 Linking with Fuzor using its API (Kalloc, 2014) 
Linking with Fuzor does not mean that the collaboration process will be in Fuzor. The system structure of ModRule need not be changed. Homebuyers will interact with ModRule and with Fuzor at the same time, as visualization, information and data will be in real-time sync with Fuzor (Figure 61).
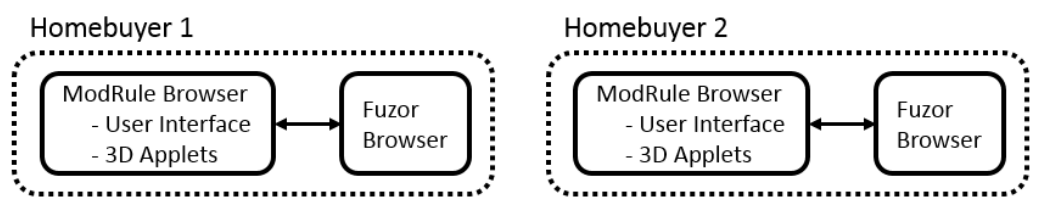

Homebuyer 3
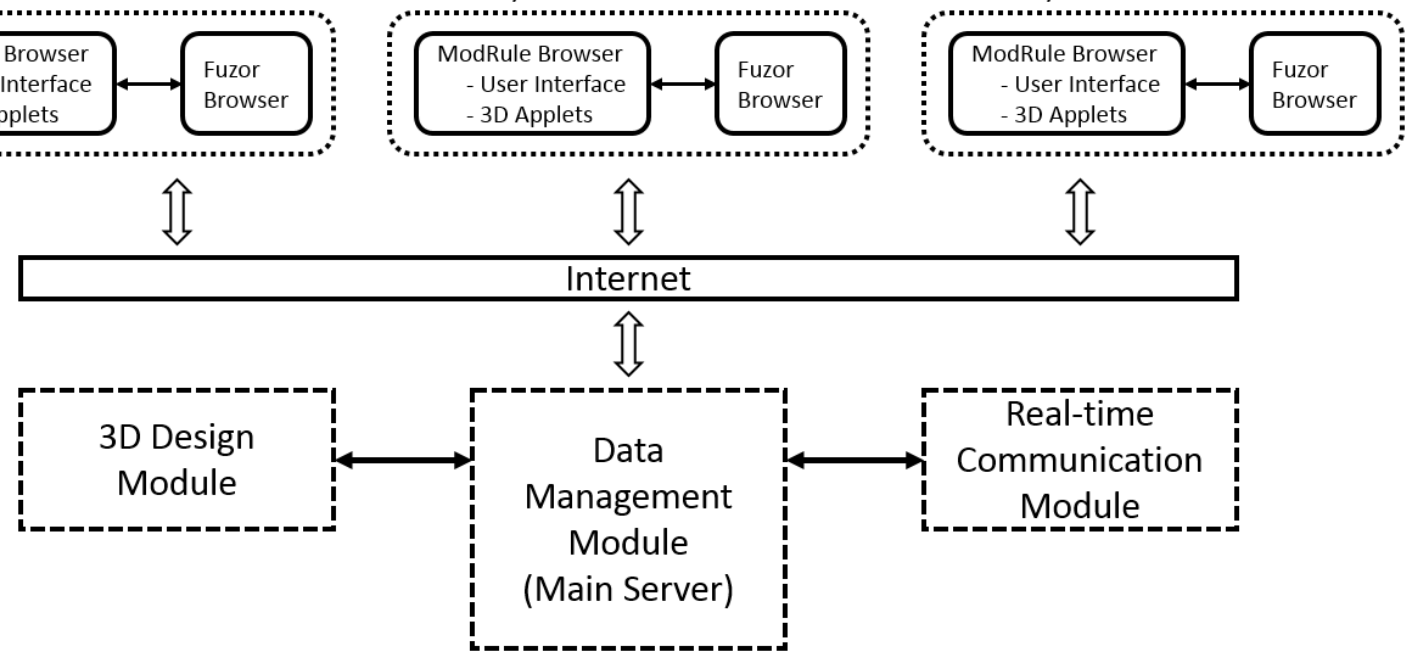

Figure 61 The system structure of ModRule with Fuzor showing only an additional browser

Another factor contributing to the use of Fuzor is its capacity to support a virtual reality (VR) experience beyond visualisation. One of the students suggested that if the design process could break out of just interacting with a computer screen, it would be much more enjoyable. The use of VR allows an immersive experience that could further motivate homebuyers to engage with the system.

\subsubsection{EXPERIMENT WITH MODRULE}

A final experiment with the ModRule-Fuzor system was conducted in an open environment where the public had a chance to trial the design system. It was conducted in the national museum of New Zealand in Wellington, known locally as 'Te Papa Tongarewa' or Te Papa Museum. Te Papa extended an invitation to showcase the design system. As the audience would be the general public, and the organisation consented to use the showcase as a form of data collection, this was an excellent platform for testing the system. 
The design framework is very similar to the study of ModRule where a building form is prepared with its grid and core space allocated, and parameters are inserted into grids. However, the setup of the experiment was very different to that of the previous two studies. Instead of having all participants sitting in front of a computer, the design process was separated into two parts (Figure 62). The first saw interested participants engage with ModRule to set up their profiles and objectives and have the spatial negotiation with other participants. Every participant engaged ModRule in the 'Collaborate' interface, with a screen displaying the 'Watch' interface linked to Fuzor within the site environment (Figure 63).

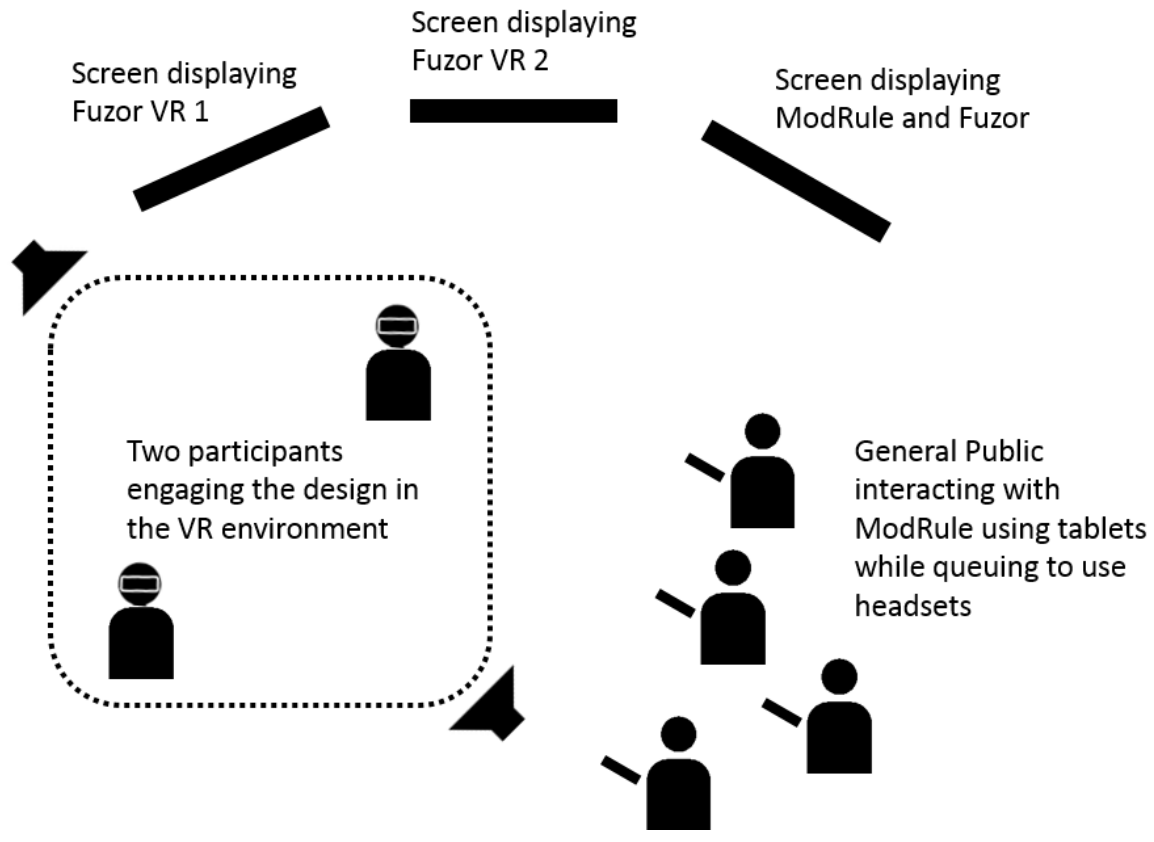

Figure 62 Setup of the beta testing of ModRule-Fuzor system 


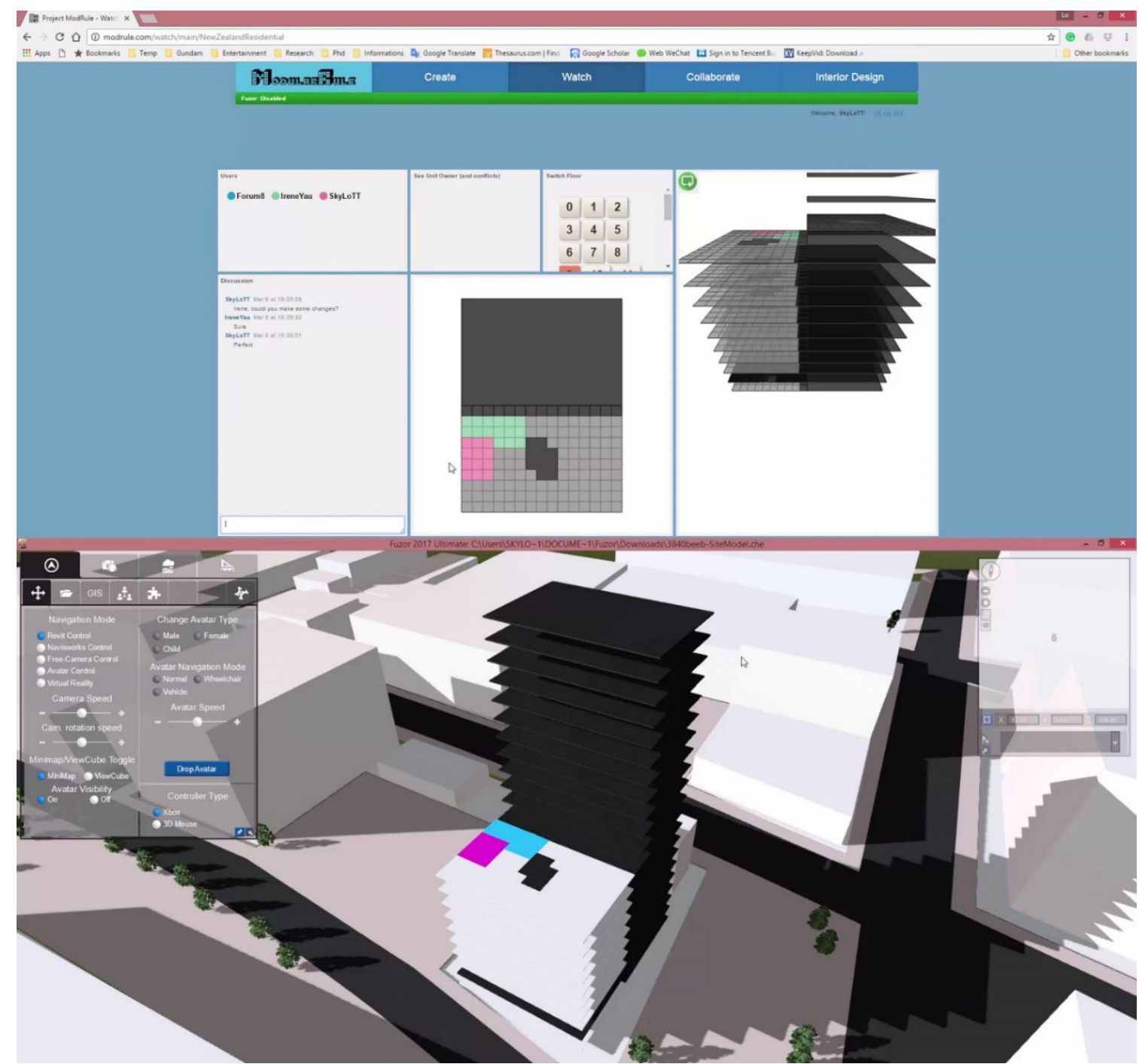

Figure 63 Screenshot of the big screen showing the participants the options they chose in ModRule (top) with respect to the site environment shown in Fuzor (bottom)

The context was a site beside Te Papa museum so the participants could be more familiar with it. The second part of the design process allowed participants to engage in the VR environment to design the space they chose for themselves (Figure 64). The participant could explore the site as if they were really there. A list of components such as walls, doors, windows and furniture were prepared and put into Fuzor for the participant to use to build up their space. Limited equipment and space meant only two participants could engage with the VR environment at one time. This, however, gave the other participants more time to interact within ModRule while they waited for their turn to be immersed in the VR design environment. Fuzor also allowed interaction within the VR environment, and each participant was able to see what the others were doing and collaborate as necessary. 


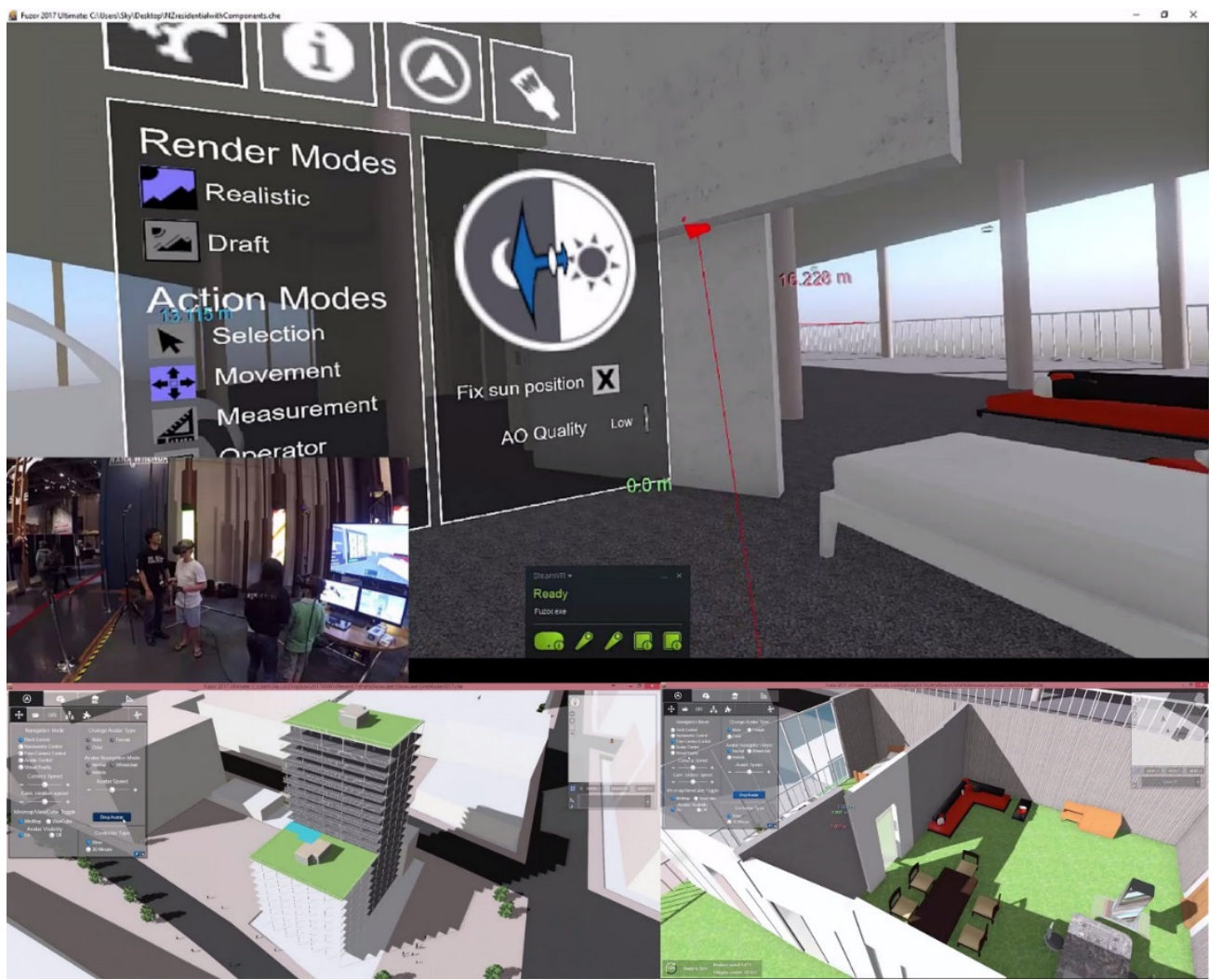

Figure 64 Screenshot showing one participants placing a wall in a VR environment (top) with a selected site (bottom left) and a prepared outcome to show its possibility (bottom right)

\subsubsection{RESULTS}

Although not real homebuyers with real needs, this test provided a general understanding of how people would react to the design system and the collaboration process.

The engagement provided by the integrated ModRule-Fuzor system was much richer than that of the previous study. The participants were highly motivated to engage with the design process even though they are not real homebuyers. The experience itself was attractive enough to motivate participants to build a design space. However, as this showcase was only for three days, there was limited time for every participant to engage with the whole design process and fully build up their space. There was no completed space as an outcome. This test therefore focused on the participants' engagement with the design system.

\section{Feedbacks of Participants}

The interface of ModRule was simple enough for participants to interact with, and the connection with Fuzor provided them with sufficient knowledge to better understand the 
context. More time was spent on teaching the participants to build up the space in the VR. Once the participants understood the controls, they were totally immersed in designing the space. There was a total of 73 participants over the three days, including twelve professionals who were in a field related to architecture. An initial survey given before participants began engaging with the system enabled a rough profile and an understanding of their spatial preferences within a house. A post-experiment survey given after participants trialled the whole design process sought feedback about their experience. More than $80 \%$ of all the participants stated their preference for this design process and that they would like to use it to design their own home. A handful found the process confusing or preferred a ready-made house. One of the participants who was really interested in the system gave very detailed feedback and commented that:

"The process is a good idea, but the execution could be better.

A little bit unintuitive in its use, perhaps because it is such a new idea/concept to understand. Would be much better if options were categorised, if you could allow change colours/textures/fabrics etc."

And when asked about his preferred method of determining his living space, he strongly preferred this system over the traditional method of looking at plans:

"This [ModRule] would definitely be a better way of visualising and interacting with the future home space."

This chapter describes the development of a digital tool for this research and the experiments performed at various stages to understand how a digital tool can engage homebuyers collaboratively. The results of each study indicated that the tool, ModRule, did provide the means to achieve the objective of this research. The next chapter further analyses the observations made in the studies and better illustrates how the research question can be answered. A metadesign framework based on the various concepts from the literature review is also established to provide a general guideline for collaborative housing design processes using digital and gamification techniques. 


\section{Chapter 6: Establishing a Mass housing Metadesign}

Then, there is complexity of the world where it is the architect who is trying to participate to make it better and not the people trying to participate in the world of architects.

- John Habraken -

(2013)

After several tests with focus groups and the public, a distinct observation is that the spatial needs and preferences of people are very diverse. Spaces selected by most participants include bedroom, living room, dining room, kitchen and bathroom, but many were also looking for a study room, workshop, storage room and balcony. There were questions about the need for separate dining and living rooms; some stating that the kitchen can be integrated with the dining room. A rare few were also hoping to have a flexible exterior to enable their unique outlook. Simple interior configuration may not work to achieve the unique functions some participants were looking for, especially in prefabricated units in apartment buildings. For example, one participant did not require a living room but preferred a workshop. A living room that is centrally located and conventionally 'fixed' in every housing unit cannot be simply converted into a workshop. Its central spatial location is not well-suited to that function. A configuration including a workshop therefore necessitates planning from the beginning.

The participants' spatial needs are observably very rich. It is almost certain that it is impossible to draw any conclusion or pattern that could help architects to design an optimal 'one fits all' housing unit. As this research investigates how to encourage the participation of homebuyers in the design process, it is as Habraken said, actually trying to participate in the life of the homebuyers in order to design a better living environment for them.

This chapter summarises and outlines the studies in this research to establish a metadesign framework for collaborative multi-families housing design. Metadesign is defined as "an emerging framework of practice that will enable designers to change, or create, behavioural paradigms" (Wood, 2016). It is a level higher than design; to examine the 
possibilities of "designing the design" using information technology (Giaccardi, 2005). It is an ambitious task focused on the realm where the complexity of natural human interaction crosses the boundary of what 'design' can achieve. Metadesign deals with the creation of context, to bring about a more synergistic society using information technology that includes a wide range of factors (Figure 65). It is not about producing fixed content like merchandise for consumption and profit. This research uses the metadesign concept to envision possible general structures and processes that could enhance housing design and bring about a more collaborative and synergistic process.

There are two parts to this chapter. The first explains the math, gamification techniques, game mechanics and game theory behind the ModRule tool. This connects with the literature and forms the groundwork of this research. The next section builds a framework and formularises the ingredients that contribute to the establishment of the metadesign for this research.

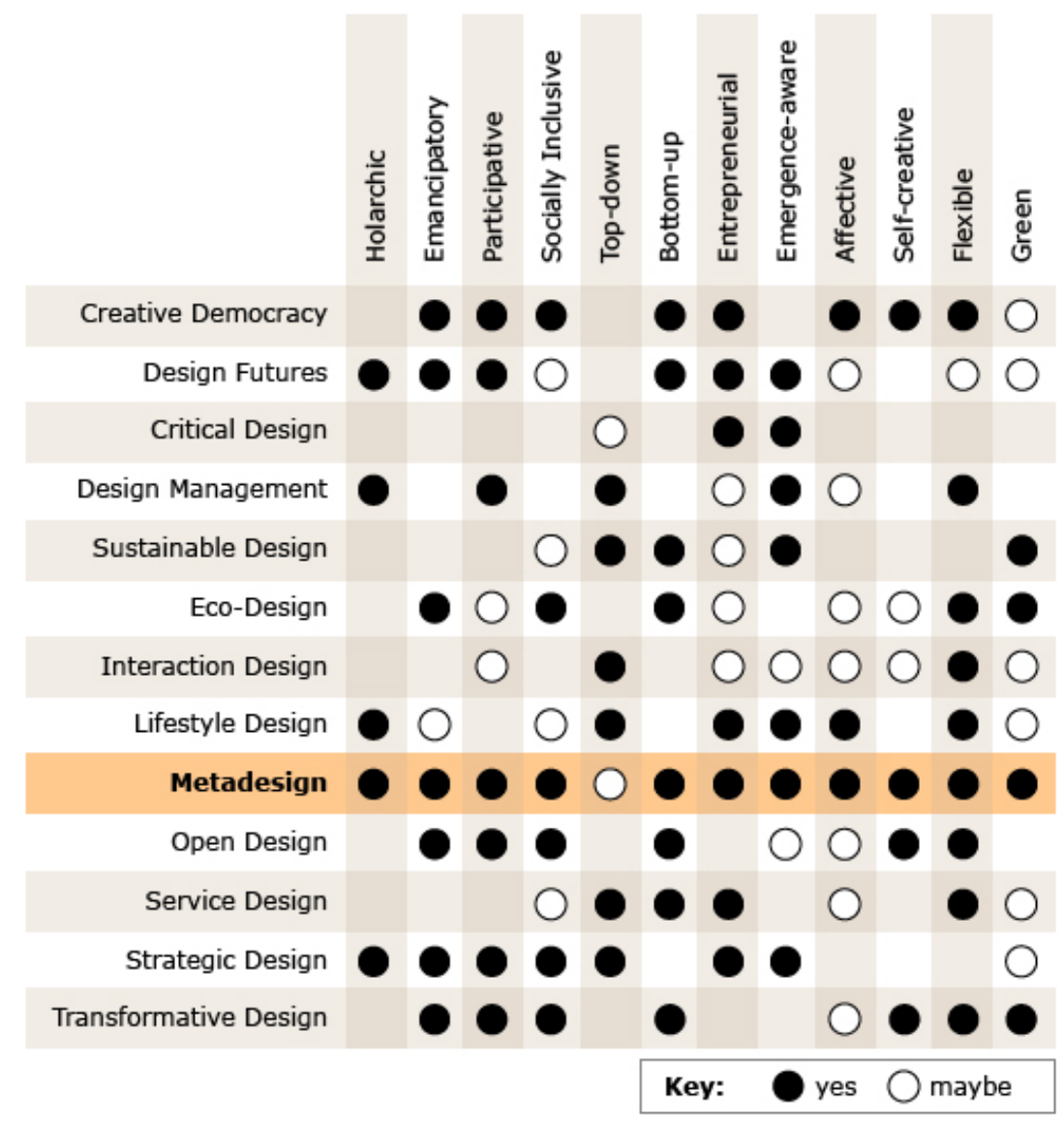

Figure 65 Schematic map indicating the character of Metadesign by Jordan Dalladay-Simpson (Wood, 2016) 


\subsection{Motivating Homebuyers}

Chapter 4 presented various approaches that could be classified as gamification and provide support and motivation for the participation of the homebuyers. Some ideas may not be obvious or are yet to be implemented in the tool, but the intention behind these is suggested and explained here for future reference.

One approach is the eight core drives by Chou, focusing on motivation (Chou, 2015). This research tries to adopt all eight core drives into the ModRule design system but, depending on features and functionalities, the use of each core drive is at different levels and some relationships with the core drive may be dissimilar to Chou's interpretation.

\subsubsection{EPIC MEANING AND CALLING}

The first drive is providing 'epic meaning and calling'. This research has nothing to do with saving or changing the world. Neither is it about big ideology like protecting humanity's knowledge as stated by Chou about Wikipedia. This research motivates homebuyers by making them part of the creation. They play a part in contributing to, and changing the built environment. It is similar to games like Minecraft and Sim City where players are not taking part in the narrative of a story but simply building stuff on their own. The difference is that the outcome is not virtual, nor just simple interior configuration; but a physical design form that will sit on the earth for everyone to witness and that they will live in. Knowing that they are participating in the formation of a building is significant motivation for homebuyers to collaborate.

\subsubsection{DEVELOPMENT AND ACCOMPLISHMENT}

The second drive is 'development and accomplishment'. In the system ModRule, there are, as yet, no PBLs implemented with which to award the homebuyers. Instead, the 'goal bar' is used to ensure that homebuyers stay within the range of their needs. It serves as the 'challenge' for them to accomplish a design outcome that is applicable within that range. In terms of development, the aim is to impart housing design knowledge to the homebuyers so they will 
understand that some demands are not necessary achievable - especially in an apartment building context. At this stage, most development is by word of mouth during the experiments. It would be useful to further enhance the system in the future to implement feedback mechanisms in the system with PBLs for homebuyers to better develop their knowledge while collaborating on design.

\subsubsection{CREATIVE EMPOWERMENT AND FEEDBACK}

The third drive of 'creative empowerment and feedback' can be easily identified. By using the open building design methodology and breaking the housing design into various components (chapter 5), the flexible design framework allows homebuyers to fully utilise their creativity to design anything within the set parameters. This can be observed in the test studies as students and participants were fully engaged in the design process to demonstrate their creativity.

\subsubsection{OWNERSHIP AND POSSESSION}

Similarly, the fourth drive of 'ownership and possession' is also easily observed. As the end result is the homebuyers' future living space, they are motivated to design and collaborate to achieve the desired outcome. It is the desire to design their own housing that drives homebuyers to participate and collaborate in this design process.

\subsubsection{SOCIAL INFLUENCE AND RELATEDNESS}

The fifth drive, 'social influence and relatedness', is achieved by having user profiles and homebuyers' goals visible to everyone. This brings about an understanding among homebuyers that everyone is designing and making choices based on their needs. It can also influence everyone to be less individualistic and to help each other achieve an overall satisfactory outcome. This can be observed in the focus groups studies in which the students helped each other reach an outcome where every role achieved their desired living space.

\subsubsection{SCARCITY AND IMPATIENCE}

The sixth drive is 'scarcity and impatience'. This drive is not implemented in this research because the participants are not real homebuyers with real needs. However, this is a drive that 
would greatly encourage homebuyers to collaborate and even compromise their needs to a certain extent. The price of housing is constantly increasing in many cities due to the imbalance of supply and demand (Global Housing Watch, 2017). If collaborating more in the design process enables homebuyers to get the house early, and before prices rise again, this would be another significant driver for the homebuyers.

\subsubsection{UNPREDICTABILITY AND CURIOSITY}

The seventh drive, 'unpredictability and curiosity', is also not implemented in the system. This is the drive most difficult to implement in this research. It is more suited to games, where it encourages players to complete a game to reach an unknown ending. In housing design, it is better for homebuyers to predict their own outcome and work towards it. However, this is not a conventional design process with a conventional outcome. This fact could motivate homebuyers to anticipate the design process, especially during the collaboration process when every design comes together to form the final outlook of the building.

\subsubsection{LOSS AND AVOIDANCE}

The last drive, 'loss and avoidance', is very similar to the sixth drive. The implementation is not yet in the system, but it can be another drive to motivate homebuyers to collaborate. Loss and avoidance can be implemented by having homebuyers who are choosing the same space, or who are 'in conflict', compete in the system. The one who collaborates with the neighbouring homebuyers wins. The math underpinning this is explained in a later section of this chapter.

Although the core drives are listed separately, they work together as a whole system to motivate homebuyers. In summary, ModRule can be seen categorised as shown in Figure 66. 


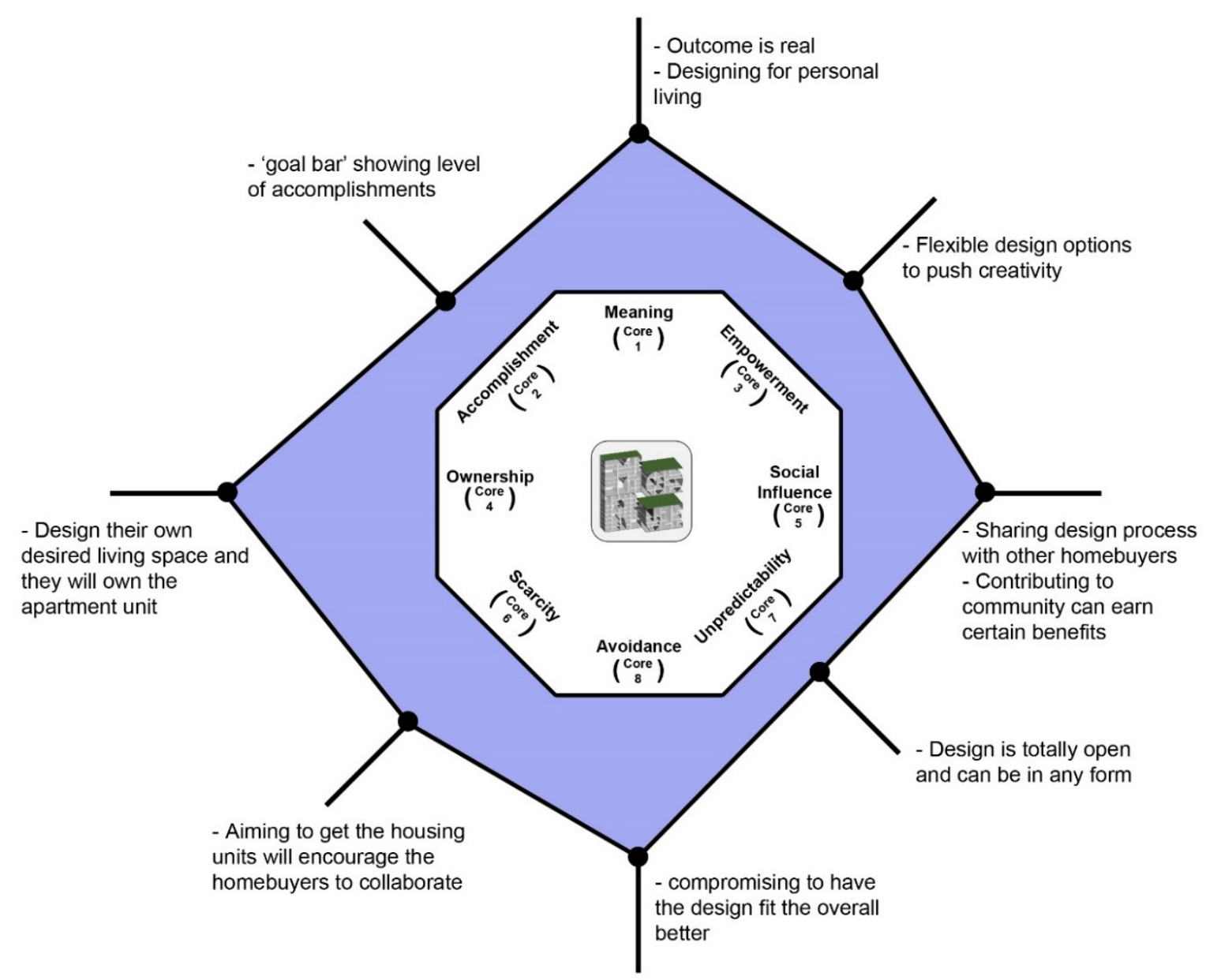

Figure 66 The level of implementation of the various core drives in ModRule, the further distance the points are from the centre means higher level of implementation to enhance engagement with homebuyers

\subsection{Entertaining Homebuyers}

This section demonstrates how the system is intended to be implemented in such a way that interactions will not be too boring. Even with every motivation drive introduced into the system, if the physical interaction with the system (which can also be known as the ninth hidden drive, 'sensation') is not attractive enough, it might not work. From this perspective, our research explores how the three rules of game design mechanics (Salen \& Zimmerman, 2003) formulate these relations and support a collaborative housing design model. Various games provide a better picture of comparing and analysing these elements.

\subsubsection{INTEGRATING CONSTITUTIVE RULES}

First is to examine the constitutive rules or underlying structure that informs the operational rules. SimCity and Minecraft are two computer games frequently utilised in relation to 
architectural tasks (Sanchez, 2015). Although they are designed as open-world games where players are required to design everything from 'tabula rasa', the players are confined and limited by rules and logic such as working in a grid environment and using building elements provided by the system. In comparison, the context of the system in this research is highdensity mass housing. There could be an open site context with surrounding buildings, streets and environment to explore but the main interaction is within the designated building. In other words, the design process is bound by the confined housing design.

In another type of game, Role-Playing (computer) Games (RPG) such as Mass Effect and Until Dawn, follow a storyline and require players to make decisions that change the course of the story, resulting in unique endings for each player. The players simply make choices. They are not required to keep score or follow complex rules. The story is adjusted according to the choices made by the players, resulting in different story endings. Such a decision-making method could assist greatly in user-oriented housing design. The architects could prepare a framework mapping out every decision leading to the outcomes they want (Figure 67).

\section{Game}

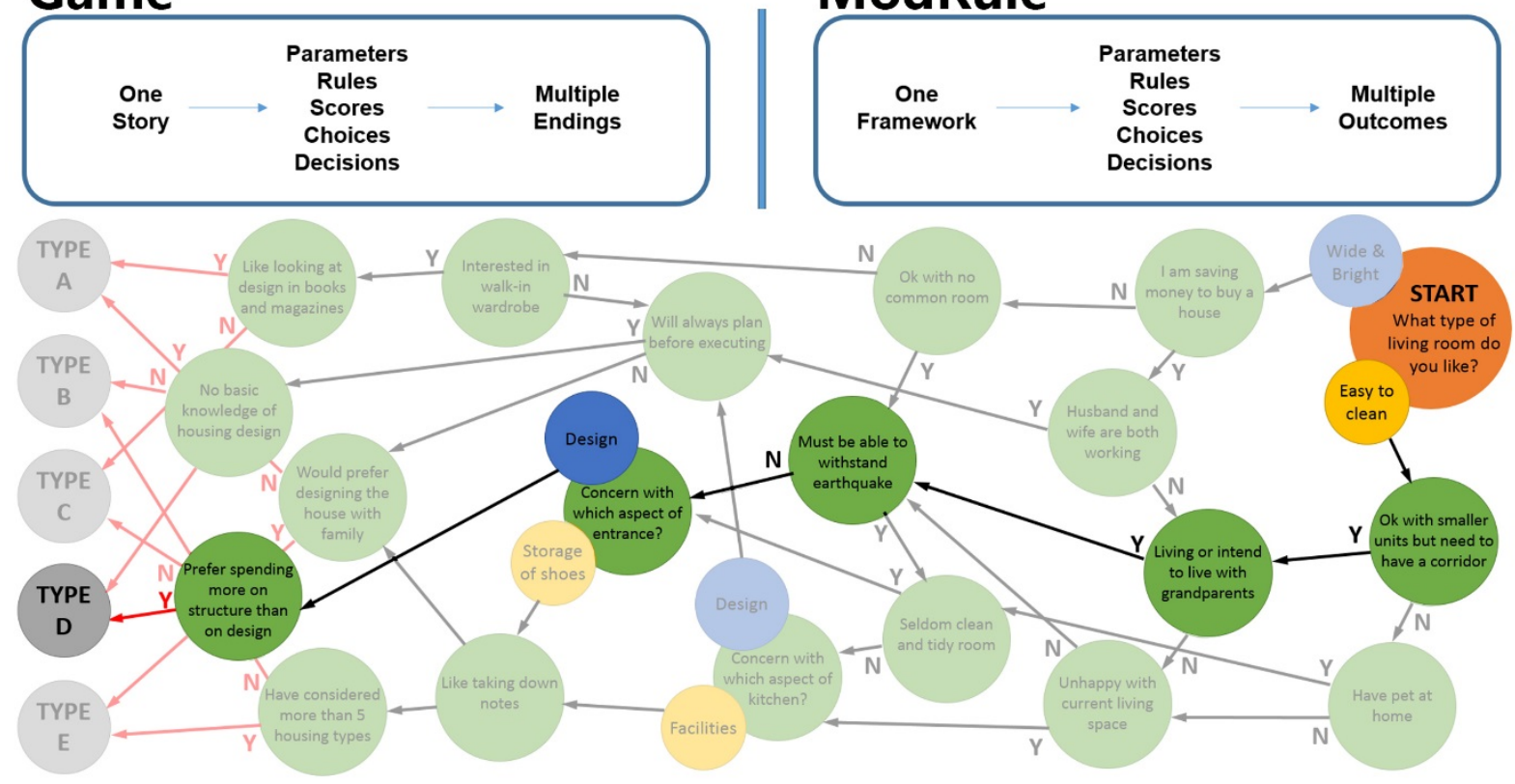

Figure 67 The overall mapping of the possible decisions in ModRule. A highlighted path showing one possible set of decisions made by a homebuyer leading to a design option (in this case Type D) 
The roles of the architect and the homebuyers also greatly affects the constitutive rules. The homebuyer may be an individual, a couple, or a family settling in an apartment. Their objective is to design a desirable outcome. Therefore, they have more limitations than the architect who is the creator of the whole context. Another example to better illustrate this point is Super Mario Maker, a game which allows players to play and also to design and build their own game stage. This breaks the traditional form of playing where players try only to beat the levels designed by the game designer. In the maker-game, the player is open to more constitutive rules, allowing them to create more random stages for other players to play. Unlike the game designer, however, players do remain restricted to a specific structure. Close examination reveals that the relationship between game designers and players is very similar to that of architects and homebuyers. This can be further observed with the influence of different gamification rules.

\subsubsection{INTEGRATING OPERATIONAL RULES}

In the realm of operational rules, the rules that most directly influence the homebuyers, the ModRule design system is both wide open and relatively closed off. Homebuyers are given more freedom in their choices, such as layout arrangement, building materials and dimensions. They are provided with a detailed view of the site context and the building structure is laid out in front of them. They can move about freely to explore the site, building their living space in whatever manner they prefer. The process is simplified to be manipulated easily; they need only generate a spatial layout map through the digital system for plans to be generated automatically (Figure 68) for further adjustments. They can also communicate and design together with other homebuyers. The design, however, is still restricted to the grid designed by the architect. The 'freedom' of choice is restricted to the range of choices decided by the architect. More choices do not mean more freedom or welfare (Schwartz, 2003). Constraints ensure a certain order within the design system so the outcome is not chaotic. This is the reason the architect has a bigger responsibility with this system; they must assess the process and outcome, assisting homebuyers to make suitable choices. If homebuyers are dissatisfied 
but provide a sound argument and alternative design, the architect makes the final decision, affecting the process and outcome. Although this may sound tedious, the increase in the anticipation of homebuyers would also greatly build up their sense of ownership, bringing about greater sustainability of the building (Israel, 2012). In any case, the operational rules are the most accessible and understandable for the homebuyers to follow during the design process.

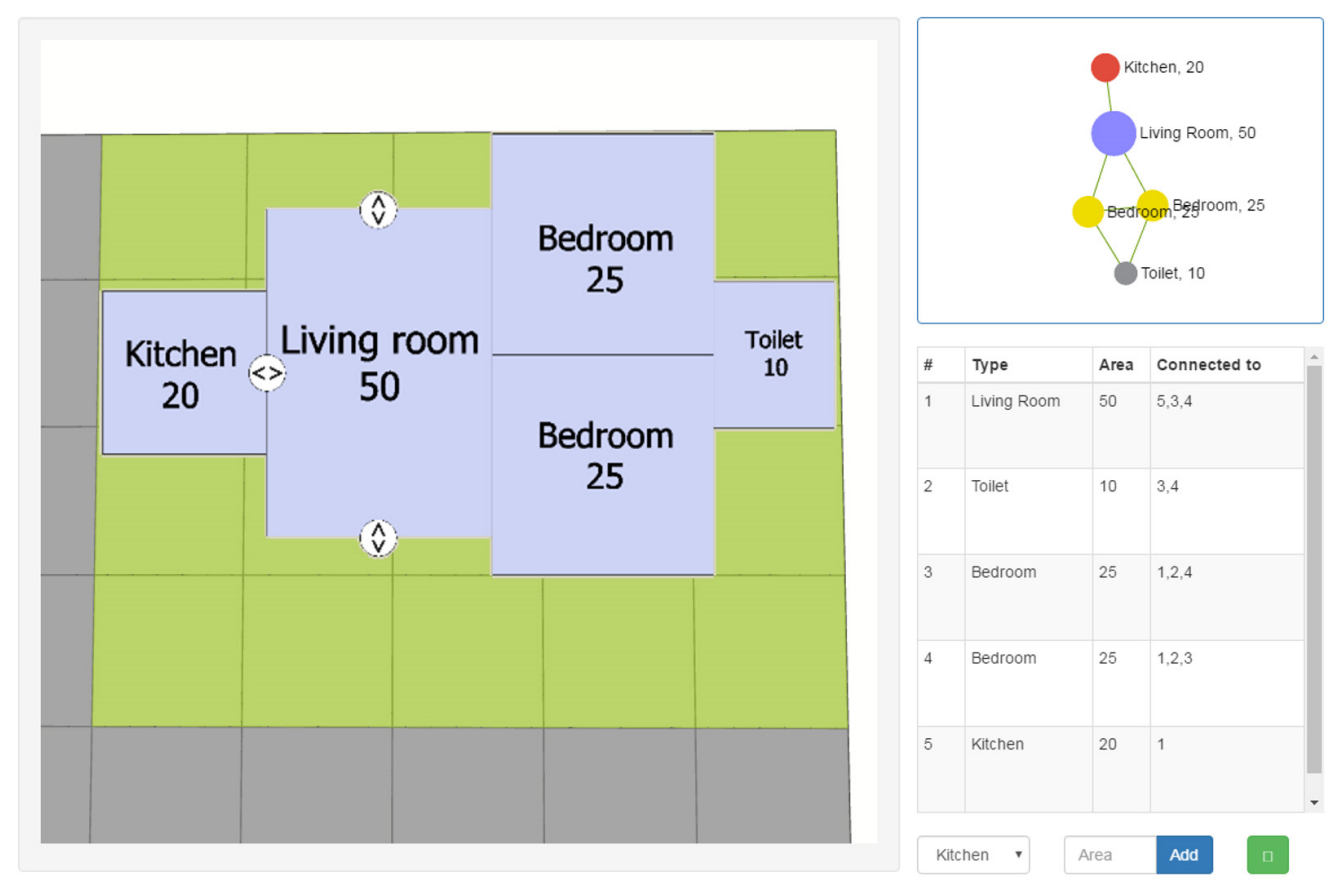

Figure 68 A tentative operation process of the homebuyers in designing their living space

There is one main gaming feature not adopted in ModRule - the objective. In games, the objective serves as a guide to players and keeps them on track with the game. This might be the main quest to advance through a story, or a side quest to enhance the attributes of the game character. This feature sits in-between the constitutive and operational rules. In ModRule, there is neither storyline nor character (similar to 'meaning', the first gamification core drive). Such a feature serves no purpose in a design context, yet an objective is necessary and in fact, exists in every homebuyer. Additionally, this 'objective' is very different for every homebuyer. Thus, instead of the architect setting a set of objectives for every homebuyer to follow, the ModRule system tailors a unique set of objectives for every homebuyer based on their needs and preferences. The user completes a set of questionnaires before accessing the 
system. This feature is part of the constitutive rules of the gamified system and also serves as operational rules as the users must comply to achieve their own objectives.

Another key feature adapted from gameplay is the 'massive multiplayer online roleplaying games' (MMORPG). At this point, this research and its proposed ModRule design system differ greatly from existing systems. Most available systems such as Wikihouse, iPrefab, and BarCode Housing for example, are designed for homebuyers to design their own houses. There is a lack of communication between homebuyers, and a lack of community design. One main reason is that including a group of homebuyers can exponentially complicate already complex housing design. However, online communities have proven, through MMO popular culture, their potential to provide powerful problem-solving capacities through collective intelligence (Segard, Moloney, \& Moleta, 2013). Games such as Clash of Clans and Mobile Strike are famous mobile application games that call for strategies among players. Players need to understand the capabilities of each teammate and communicate well to execute the best performance against common enemies. New friends and communities are formed in these games; internet forums have been created to allow discussion of battle strategies, etc. The key point here is that through a strategic process, people tend to communicate with each other to decide the strategy that suits them best, and through this communication build communities and relationships that push them towards solving problems together. There is clearly great potential to integrate this capability into an online digital housing design system in hope of bringing about a collective design that an architect would not easily generate alone. Yet, even with gamification, the whole process is not implemented easily. There is a crucial, perhaps controversial precondition; participating homebuyers cannot be anonymous as in MMORPG.

\subsubsection{INTEGRATING IMPLICIT RULES}

This leads to the implicit rules, which can be considered the most important in the ModRule design system. The design process depends on the participation of every homebuyer, and there is no specific control over their actions. Therefore, the success of this model relies heavily 
on these unwritten rules. Three key requirements for the initiation of an accurate, user-oriented collaboration were identified earlier: Communication, transparency, and understanding. Every aspect of gamification seeks to bring about these attributes among the homebuyers. Context variables such as socio-economic background, rate of mobility, life stage and living quality preferences are some necessary data to ensure this gamified design process produces a relevant result. Information comes in two levels: Level I is general information that influences design decisions; Level II is analysed information that responds to the needs of the homebuyers. In addition to providing truthful personal information, timely feedback is also necessary to determine the criteria for their selections and reduce the uncertainties of the participatory process. The aim of gamification is to entice the homebuyers into the process with an entertaining experience, enabling them to provide this information with ease and confidence.

\subsection{Stimulating Homebuyers}

The next advantage of using gamification is its potential to reduce conflict. Making use of the game theory described in the previous chapter, Modrule adopted the strategy of manipulating various parameters until all the spatial units have both pros and cons. For example, a unit on the top floor will have a great view, but also a high amount of sunlight. A unit on the bottom floor might be noisy, but have high accessibility to ground facilities. This references games such as Gauntlet Legends in the 90s. These have characters with various attributes (Figure 69) allowing players to choose the class that best fits their playing style, and to collaborate with players of other classes to bring out the best playing experience. 


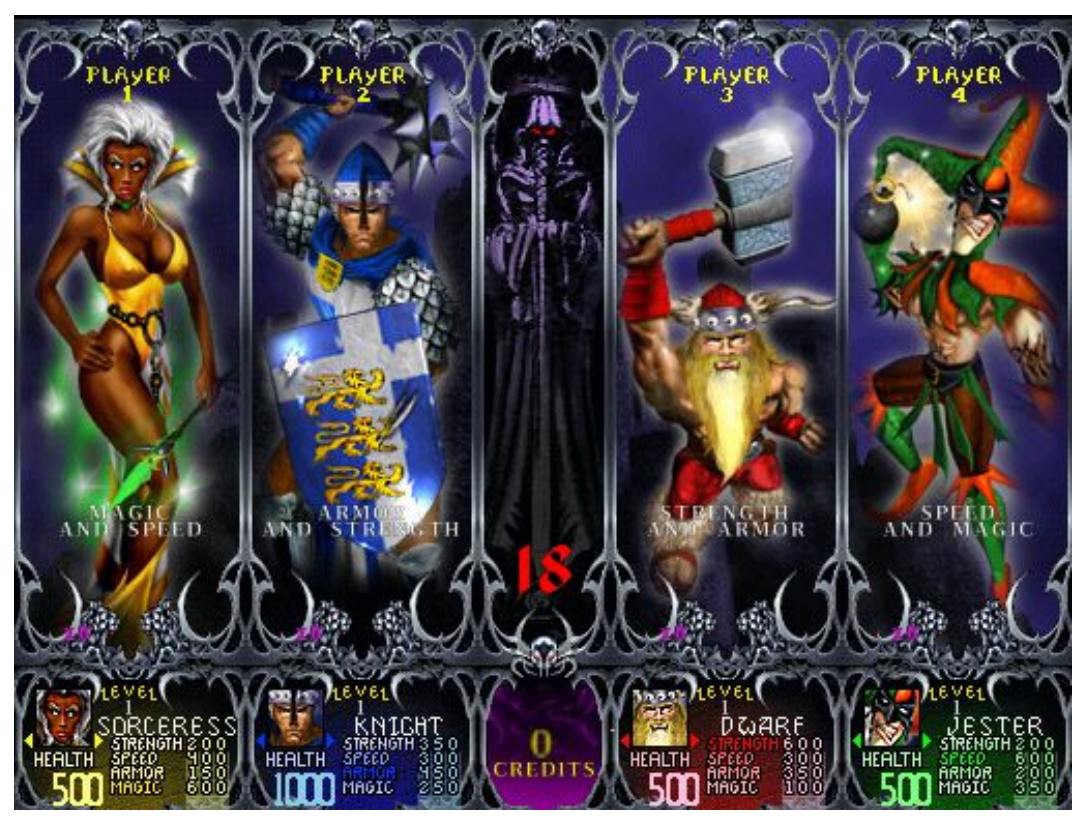

Figure 69 A screenshot of the game "Gauntlet Legends" showing the attributes of the character class (image from Pinterest)

This prevents every player from going for the 'best character' (if there is one) with all its attributes "maxed out" - making the game less of a challenge. In the context of this research, the parameters are varied as much as possible to accommodate the huge variety of homebuyer profiles (Figure 70).

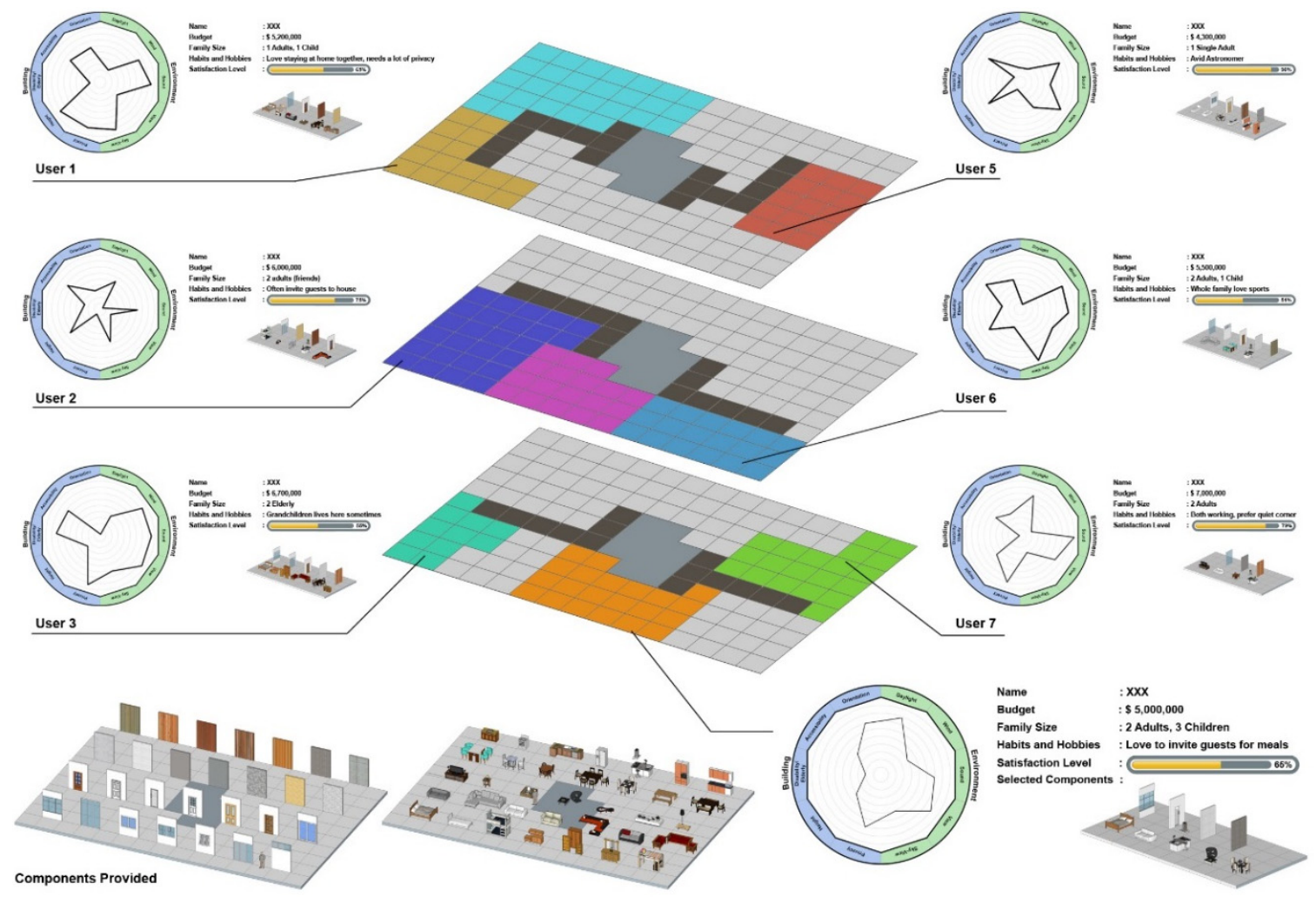

Figure 70 Homebuyers profiles created and played by participants from the study of ModRule 


\subsubsection{TOUCHING ON GAME THEORY}

All spatial units include at least one negative parameter so homebuyers do not 'fight' for the same unit. Although cost is a key factor affecting the choice of the homebuyers, they are strongly discouraged from using only that one parameter as a guide. The aim is for everyone to have the outcome matching their profile as closely as possible. In reality, however, there are homebuyers who are rich and who can afford to select the units which they prefer, but don't necessarily need. This was role-played by the students in one of the ModRule studies. At the end of the study, the suggestion was made that a 'community' parameter be added with benefits included to further encourage homebuyers to collaborate with one another (Figure 71). This occurs not only with respect to design issues but also in agreements within the community pertaining to lifestyle and mutual support. For example, if homebuyer ' $A$ ' offers to maintain the cleanliness of a given communal area, he or she will be identified as 'worth' more than homebuyer ' $B$ ' who contributes nothing to the community. In return, end user ' $A$ ' becomes eligible to receive some form of remuneration, such as 'discount per square area' or 'greater advantage in being chosen as occupants' as agreed in advance and set as part of the overall system parameters by the architects.

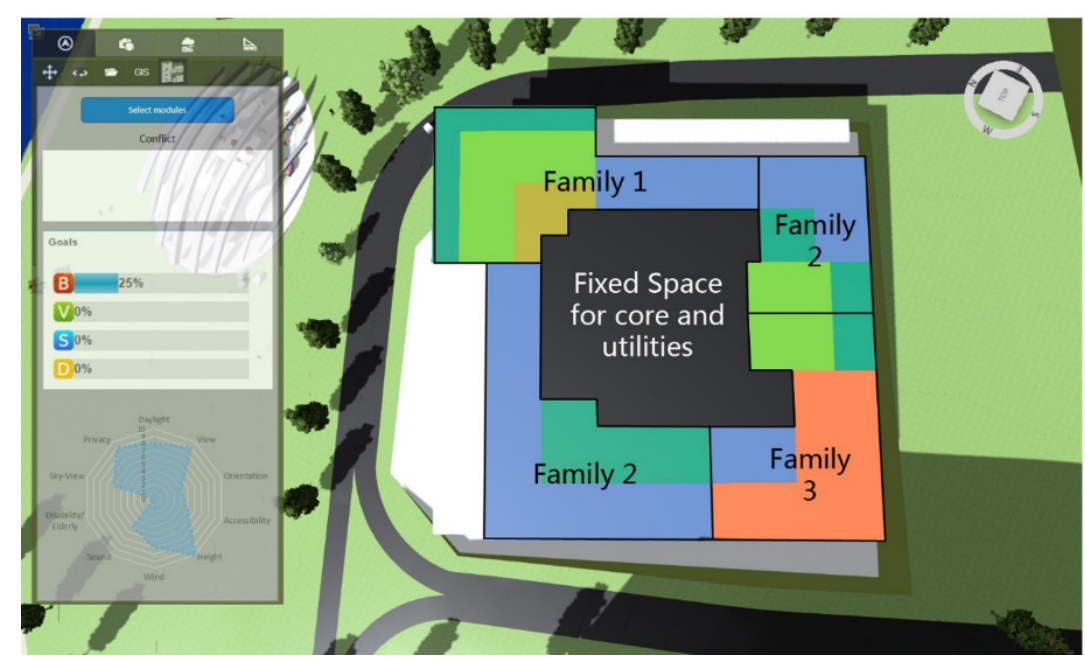

Contribution to Community

Figure 71 Suggested addition to the parameters to encourage more collaboration 
The aim of setting these parameters is to stimulate homebuyers to work together strategically to achieve win-win situations with one another. The parameters are set in such a manner that this is possible, and so that if some factors are compromised a better benefit is available to reduce the sense of loss.

It is difficult to say at this stage that these gamification factors truly motivated homebuyers to collaborate with each other. The challenges and limitations that exist in the study are further explained in a later chapter. But through the study with ModRule and engaging the public, the results demonstrate that design collaboration is achievable and highly beneficial socially, economically and potentially politically. The next step is to crystallize the design framework to help future research to manage this kind of design process.

\subsection{Metadesign: The Decision-Making Framework}

In Chapter 3, this research refers to Wood's concept of micro-utopia through synergistic collaboration among people and proposed metadesign as a desirable framework with which to achieve this. With this foundation, and through the observations made and studies performed during the tool development process, this research has come to develop a version of metadesign framework suitable for a mass housing collaboration design process, especially through the use of digital tools.

\subsubsection{RELATIONSHIP MODEL}

In every game, although the ideal players are everybody, specific categories of player will be the initial focus. Similarly, the digital housing design structure will have its targeted audience. In the housing supply/demand process, the major influential parties are architects and clients (Friedman A. , 2011) who are also the key parties in this research. One major element in metadesign as presented by Wood is a four-fold system visualised as a tetrahedral form (Figure 72). The qualities of such a system help architects to think in a self-reflexive and dynamic way. Instead of a typical two-dimensional system, this unique form is threedimensional - providing a manageable set of relations. Unlike the nodal diagrams presented in 
Chapter 3, this tetrahedron is 3-dimensional and contains four nodes and six direct relationships. This is the largest possible number of peer-to-peer relationships that can be comprehended either consciously or intuitively (Wood, 2007, p. 159). This form of nodal diagram is generated in such a way that all nodes are of similar distance between each neighbouring node, forming a 'leaderless' system. This is to exemplify a non-hierarchical relationship and enable a more collaborative environment among the parties.
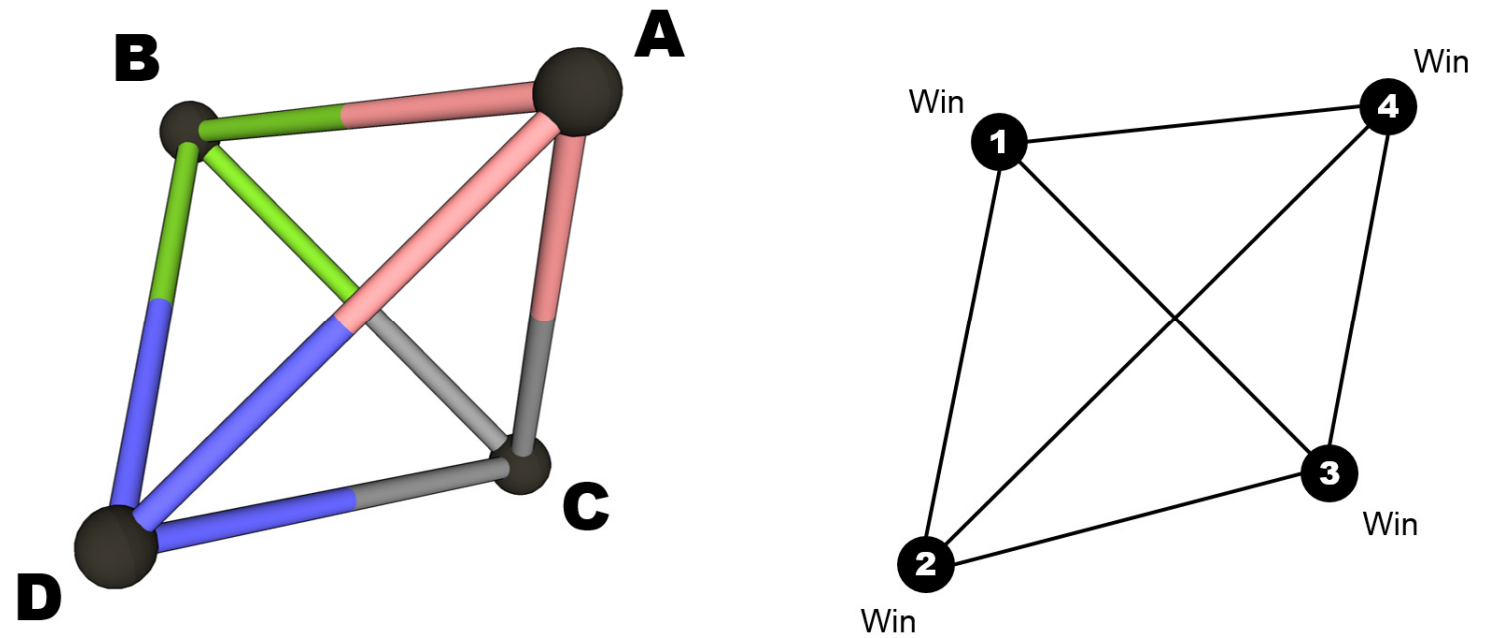

Figure 72 Tetrahedron depicting minimum grammar of relations (left) and achieving best outcome (right) (Wood, 2007)

The metadesign used here in the context of a collaborative design system, provides a convenient method of overseeing a complex problem. The components of the system are adjusted as follows:

- A: Agent - the agent is the professional, or anybody contextualised by nodes B to D. In this case $A$ is the architect.

- B: Significant action or proposition in question - the collaborative design system that is proposed by this research.

- C: Recipient - Homebuyer who is the main target beneficiary.

- D: Full local and global context of $A$ to $C$ - the housing design task which this system is designed for. 
By specifying the involved parties and components in the nodes, the relationships that need to be considered become clear. This research seeks a win-win situation for homebuyers. The metadesign model enables the win-win situation to be increased six-fold, achieving a 'winwin-win-win' outcome (Figure 72). This must be coupled with strategic design parameters, information gathering and decision making as these are the key processes to be taken into consideration.

\subsubsection{DECISION-MAKING MODEL}

The next part of the metadesign framework closely references Avi Friedman's decision-making model for flexibility (2011). Figure 73 presents the adopted model being introduced as decision support to mediate the housing design process. This model breaks down the process into two scopes of exchange, the architect with the Buying Clients setting the objectives and parameters with respect to the site context and regulation standards (A-D node), and the architect with homebuyers to determine the outcome of their desired living space (A-C node). Within the scopes of exchange, the model includes the connections of all twelve relations between the nodes. It encompasses how the digital system could assist the architect in setting objectives (B-A node), provides for homebuyers as a tool to generate alternative designs (B-C node) and brings the generated design into context for the architects to re-coordinate the overall design (B-D node).

The design workflow of ModRule started off closely following Avi Friedman's decisionmaking model for flexibility. Through the study of the ModRule tool an alternate version of the decision-making model was developed, mainly to fit the context of integrating digital tools into the design process. The digital tool allows more loops during the design process, improving the design outcome and generating more options for comparison. Design solutions are generated directly by the homebuyers, instead of architects generating a series for selection which might not suit. 


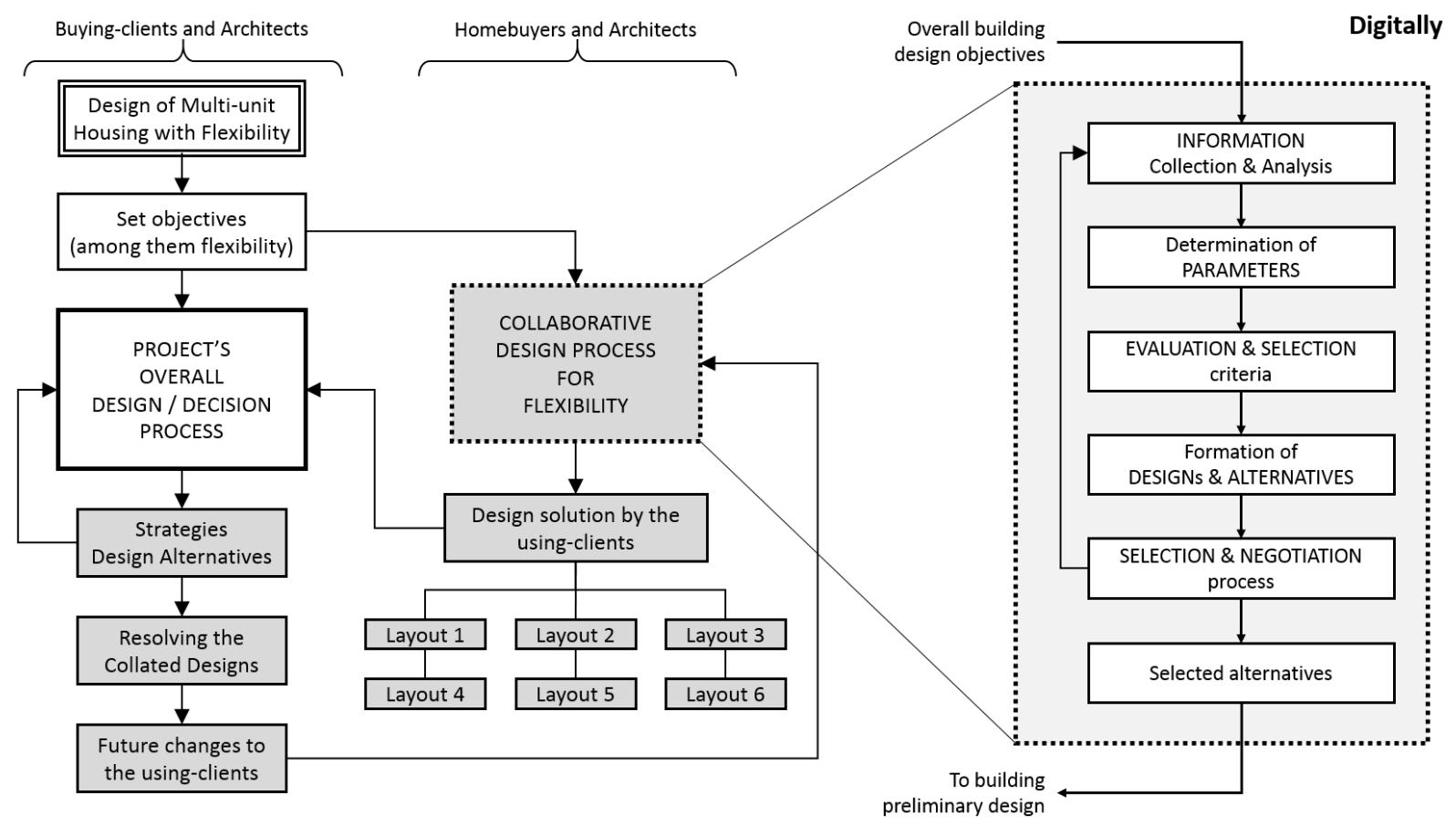

Figure 73 Friedman-adapted (2011) diagram showing the digital intervention in the housing design process as decision support

\subsubsection{CONNECTION TO BIM}

The development of this metadesign framework is based on observing homebuyers engaging with the system in the experiments of this research. However, the Buying clients and the developers that are part of the overall framework were not included in this research study. At this stage, they are categorised together with the architects as a single 'node' (being the professionals) in the metadesign network structure to retain the scope of this research. They are incorporated in the framework model as the digital design system and the framework structure could potentially be expanded so that this research forms part of the BIM family (Figure 74). 


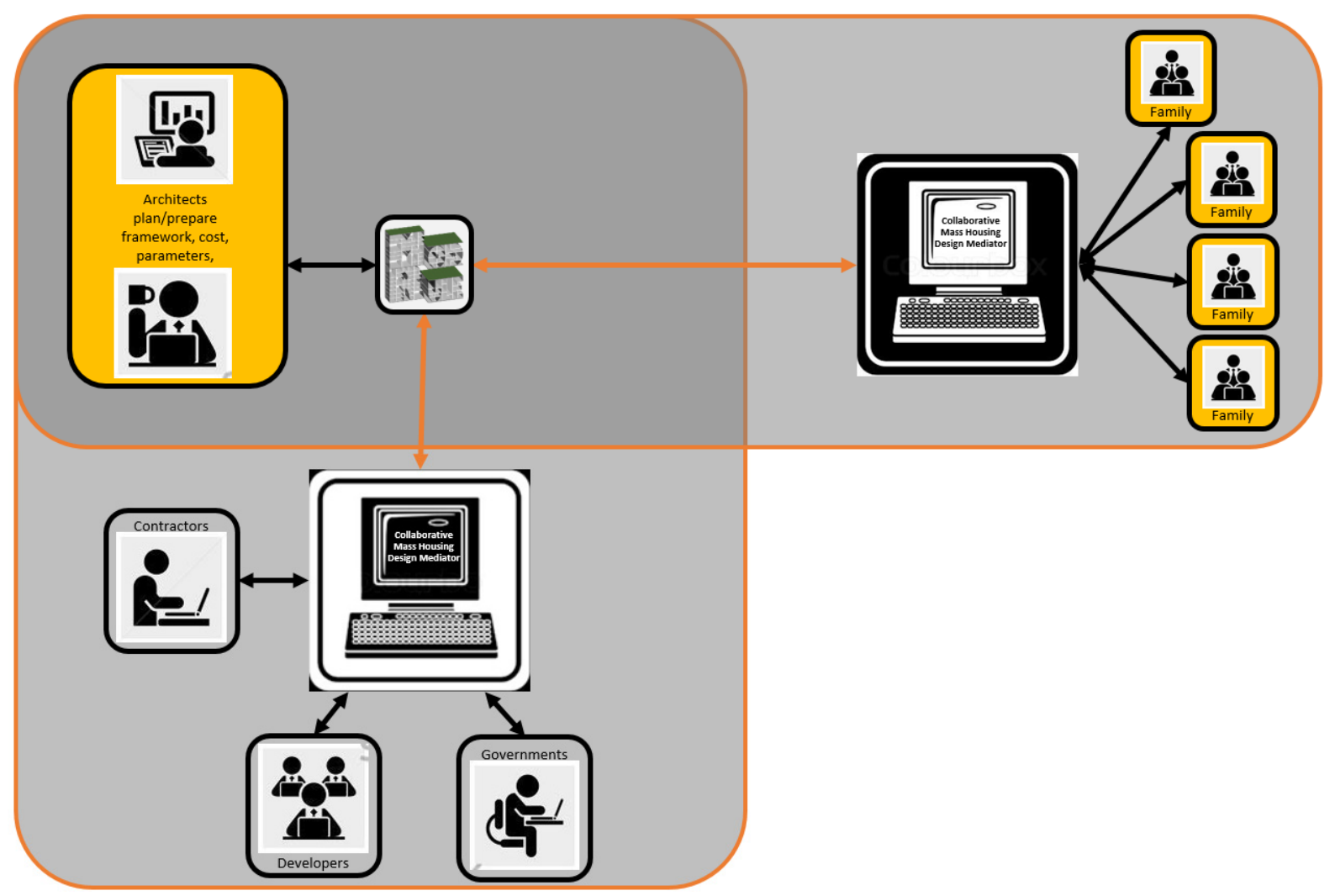

Figure 74 The possible connection of the housing design system ModRule to be expanded and connect to BIM with the professionals with architects playing the key role

\section{Summary}

The purpose of this metadesign framework is to give an overview of the design structure and better understand where this research fits into a bigger picture. It is an ambitious task to re-design design, so it is necessary to have a clear framework. This research began with a very different framework that has changed drastically over the research process to be more specific and suit the scope and context of the targeted homebuyers. Using this framework, this research can also be expanded to fit other design projects. The next chapter discusses how this research contributes to metadesign ideology and how this design framework can be flexibly applied. 


\section{Chapter 7: Discussion}

We should study the built environment as an autonomous issue, not something that we created but something that we can contribute to.

- John Habraken -

(2013)

The contribution of our research is a digital tool that mediates the collaboration of, and communication between, homebuyers and architects. Our intention is to materialise Habraken's call for 'a contribution to the built environment' (Luthi \& Schwarz, 2013), not as individuals but collectively and collaboratively. Here, the 'built environment' is enhanced within the scope of the housing implementation design environment, which is digitalised through ModRule. In this chapter, we summarise our body of research and discuss how the development of ModRule, and the use of gamification, has met the objectives identified at the beginning and achieved the aims of this research. We also explain how the metadesign can change the housing paradigm to better include the homebuyers in the design process.

\subsection{Contributing to the Housing Implementation Design Environment}

Through focus group studies, we have identified that by using a digital platform to enhance the interaction and design engagement between homebuyers, and with the architect, there is a great opportunity to improve the housing implementation design environment. The improvement can come in the form of enriching the dynamic outlook of housing design, by breaking the trend of over-modularity of mass housing with a collective and collaborative design outcome. Another contribution is to homebuyers; guiding them through collaborative design and, in the process, providing them knowledge of various aspects of housing design. The homebuyers become more aware of design features and this leads them to more conscientious decision-making while collaborating with one another. 


\subsubsection{BRINGING CHANGES TO THE BUILT ENVRIONMENT}

The outcomes generated by the studies are observably as vibrant and dynamic as the Ökohaus and La Mémé. Constraints such as materials and geometric parameters control the outlook of the design to avoid a patchwork of individual styles. A consolidated outlook is also due to the presence of a design coordinator (the architect in practical context) who keeps track of the design process. Richard Rogers (2012) stated that architects no longer work alone, and that the client is the most important part of the team. He added that good design is driven by clients who understand their aims. Although the 'clients' referred to by Rogers are developers, it is possible for homebuyers to drive good design if they also understand their aims. The challenge in this context is to support the homebuyers to articulate their design needs and merge their aims collectively. With digital tools and the architect, this approach can ensure that designs reflect homebuyers' needs and contribute to changing the built environment. Although the outcome from ModRule may still require some optimisation forces to generate a valid design, the studies show a positive trend for the digital tool to provide the necessary support for design collaboration in the context of multi-user-oriented housing.

\subsubsection{MORE THAN COLLABORATION}

To maintain a sustainable built environment, Wood (2007) established that one should 'act cosustainably with a degree of self-restraint'. Instead of just indulging an individual's desire, it is necessary for everyone to attune to the immediate present (Wood, 2007, p. 194). Current systems are overly focused on the second order of synergy, where data is processed to become potentially useful. That is not enough to extend the boundaries of the current housing design implementation model. With society becoming more digitally connected, the challenge to share and understand each other is becoming more necessary to accommodate diversity. Mass housing, therefore, has great potential to be the vehicle to bring about this phenomenon. Through the capacity of a digital design system such as ModRule, the ease of communication and the desire to design a personalised living space can encourage the sharing of information and knowledge among homebuyers. By involving homebuyers in the design process and 
enabling them to collaborate with one another, this bottom-up initiative can achieve the third order of synergy that we are aiming for. We observed participants sharing their life experiences while designing their houses in ModRule; discussing their lifestyles, how they use certain spaces, and the problems they want to tackle and improve. The conversation brought new knowledge to the participants and coordinator, knowledge that aided the progress of this research. The housing implementation design environment can see new reforms if such collaborative progress can be stimulated automatically through the design process.

\subsubsection{CHALLENGES FOR STANDARD ARCHITECTURAL PRACTICES}

However, the process is not all positive; depending on the parameters set in the design framework, it could yield a negative outcome. In the first focus group study before the development of ModRule, the group with fixed parameters imposed by us in the design brief generated a very individualistic outcome, with little evidence of collaboration. There are additional contributing factors, for example incompatible personalities and irregular work schedules of participants, however these factors are also observed in the other group. The main reason for a lack of collaborative design success is because the participants became frustrated and bored with the fixed parameters, so lost interest with the process after a short period.

With that mentioned, we have also established that some architects might not be able to accommodate this kind of design process. This leads to design unsuited to the participation of homebuyers. Architects are the key personnel who determine the parameters. Without architects who are open to constructing a design framework that is capable of such flexibility for homebuyers, the process will be fraught with controversy. This can be observed in one of the participants in the study of ModRule explained in Chapter 5 (Figure 60). In other words, if the A-D (architect - context) relation is not established properly, the A-C (architect homebuyers) relation will crumble and initiate a chain reaction, leading to the failure of the whole metadesign structure. If, however, the housing design parameters used in ModRule are 
set accordingly, the outcome is an advancement that could break out of the current housing model and improve the condition of the urban built environment.

\subsection{Mediating a Complex Design Operation}

As observed in the public engagement activities integrating ModRule with gamification techniques, four factors are identified that could help mediate complex housing design operations among homebuyers. These include enhancing design communication, simplifying the design process, adding motivational factors, and formulating the decision network.

\subsubsection{ENHANCING DESIGN COMMUNICATION}

In Chapter 3, we proposed that a digital design tool is more efficient, and most importantly, allows the architects to have a different relationship with the design process and outcome. ModRule incorporates those functions and extends them to enable homebuyers to be involved in the design process.

By profiling homebuyers, the system can help them to better understand their own needs and those of the other participating homebuyers. The profile information can also help architects to better connect with homebuyers to generate a desirable outcome. Another advantage is that the data can also be used by other professionals, notably government bodies, to keep up with the changing needs of the people.

Although communication takes place within a digital environment and is perhaps less efficient than face-to-face communication, it is much more manageable and time effective. This research finds that homebuyers and architects able to communicate with each other during the whole design process generate design outcomes more fitting to the homebuyers' needs. While initial ideas may be inappropriate, through communication with architects homebuyers can learn possibilities and constraints and generate a design which is out of the box yet within design constraints. 


\subsubsection{SIMPLIFYING THE DESIGN PROCESS}

With respect to Wood's steps toward micro-utopia, the simplification of the design process provided the necessary means to achieve the first step in breaking the psychological barrier of the homebuyers. As we observed, participants are more willing to provide the details of their desired living space and give suggestions to improve the environment. The second step was achieved to a certain extent as they were comfortable coming together in the system to collaborate and discuss how they can accommodate each other's needs. Participants are more satisfied when they accommodate all their needs within the design parameters. 'Goal bar' provided a tangible guide to direct the homebuyers towards a common goal, the basis of steps three and four. This can drive a more objective-based design discussion in that homebuyers can visualise the process of exchange and make it more efficient. This then leads to the last step in motivating the homebuyers to work together to share the tasks of producing their future home.

The studies undertaken have demonstrated that the term 'gamification' used in this research is very different to how others use it for monetary benefits. It is a method that this research adopted to achieve its aim in a much more intensified manner. This method of adopting gamification is based on what collaborative housing design is missing, especially in the context of involving homebuyers. The factors that are identified as necessary for the development of an engaging digital tool suitable for collective design in a high-rise residential context can be mostly found in the concept of gamification.

To provide better experiences for homebuyers, ModRule is also expanded to provide 3D walkthrough experiences either through a monitor, like a computer game, or in virtual reality through a headset. By making the design process open and flexible where architectural components are broken down into units, homebuyers can manipulate the entire living space directly. The public engagement activities undertaken with ModRule-Fuzor provide great insight regarding the use of enhanced visualisation. Although the interactive elements of the interface will benefit from improvements to make the operation more intuitive (based on 
participant feedback in Chapter 5), most participants welcomed the introduction of improved visualisation. Most importantly, the design feedback is instant and, with the presence of an expert, homebuyers can understand how their choices and decisions affect the other homebuyers and impact the overall design outcome.

\subsubsection{ADDING MOTIVATIONAL FACTORS}

The motivational factors inspired from Chou (2015) and integrated into ModRule work in parallel with the design simplification to play a significant role in enabling homebuyers' engagement with the design process. We have established that the use of motivational factors in digital tools improves the involvement of the participants and the collaboration among them and encourages them to change the built environment to accommodate their needs and preferences, inspiring them to do more to create a mutually beneficial, shared built environment.

We observed in the studies that giving ownership to people encourages them to take care of the commodity, even if it is just a virtual object. When provided with more design flexibility, the participants from the focus group study took great ownership of the outcome even though they are not real homebuyers. Design flexibility enables participants to manipulate the interior floorplans and the exterior outlook of the overall mass housing building. As the exterior significantly impacts the built environment, the participants work closely with each other and the architect to generate a contextual design. Most importantly, the exterior of the building publicly showcases their design creativity. This encourages participants to be conscious of the outcome and to collaborate with each other to make sure their design is not too bizarre. The proposed new method of designing housing collaboratively matches monetary factors in driving participants to spend sufficient time and effort collaborating and designing to generate a detailed model with which to showcase their design (Figure 75). 


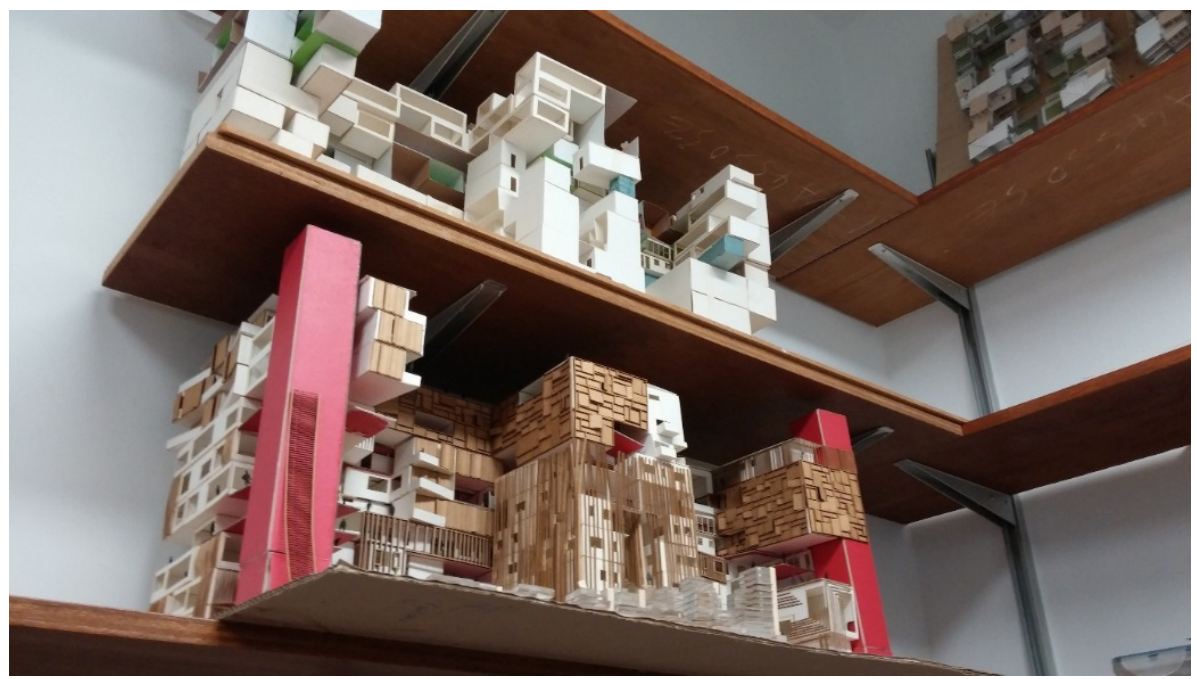

Figure 75 Model built by one of the groups among the participating students

\subsubsection{FORMULATING DESIGN DECISION SUPPORT NETWORK}

After experiencing the design process with participants in focus group studies and public engagement activities, there is one factor that we consider to be very important; providing decision support. Guiding homebuyers to make rational decisions is as significant a role for architects as helping the homebuyers to articulate their design needs. Taniguchi (2012) stated that homebuyers do not have any idea how to express their design needs. A useful method is to ask questions about their current living conditions and slowly build up an impression of the desired design. To mitigate the tedious process of interviewing every homebuyer and sketching their ideas in bits, a decision support network (Figure 67, page 148) can be integrated into a digital tool to match the homebuyers to a housing type which can act as a 'starting point' for them to begin designing. Decision support is, however, yet to be fully integrated into ModRule. Most of the time during the studies, we provided decision support and helped participants to articulate their design, leading them to the collaborative stage of the design process. Still, the studies indicate that a design decision support network is crucial for homebuyers to engage with the design process.

\subsubsection{CHALLENGES TO THE SYSTEM}

Understanding the scale of our aspirations, the system can be considered unfinished and with many possible improvements. Although it can be observed that participants are more willing 
to participate in the design process, the interface is insufficiently user-friendly to allow homebuyers to pick up the design tool intuitively. Understanding how the interface can be improved to work with homebuyers is another research process to be furthered. By using webGL, ModRule only provides an abstract model of the apartment building. It is only after linking it with Fuzor that the connection to the real environment is enhanced. The controls of the interface can also be greatly improved to visually attract homebuyers to interact with the system. Additionally, although results are positive the limited number of participants do not guarantee that the results can be extrapolated to the complexity of a high-rise apartment in which the number of participants might increase exponentially and result in a totally different outcome. Nevertheless, this does not detract from the significance and contributions of this research. The focus group allows for effective design exchange and observation. Some separate workshops and small seminars were also conducted with fewer participants and the process and results did not differ much from the research experiments (Lo et al., 2016, Lo, 2016). We speculate that even if the number of homebuyers increases further, provided the system can hold the data exchange, the outcome should be somewhat similar. Further research into large number design collaboration is definitely necessary, especially with laypeople, to fully comprehend the capacity of the design system and framework described in this research.

\subsection{Changing the Paradigm}

A metadesign framework is developed from the studies to contribute to this body of research. One important factor that we are trying to achieve through the collaborative design framework is the synergy that Wood described. This is where the paradigm shift will occur most notably.

\subsubsection{CHANGING MINDSET}

In the process of engaging the participants to study the design collaboration, we used gamification as a method to envision housing design collaboration in a different perspective. This method is new but not unfounded. Ever since the game Minecraft became a phenomenon 
in 2010 (alphr, 2015), many have used it for other purposes, such as education for example (Duncan, 2011). If one takes a closer look, the game is very old-school and nothing like contemporary, best-selling games with high-quality graphics and storylines (Echo, 2014). It is the ability to build a world from scratch through the simple processes of mining and crafting, as the title suggests, that allows players to make use of their creativity and, most importantly, share it (Lastowka, 2011). Games in this genre are not new; they are generally called 'voxel games' or 'sandbox platformers' which are open worlds allowing players to create anything within a virtual environment. One such game is Game Zero which was created before Minecraft but failed to become a sensation at that time (Perry, 2016). The point here is that people's mindsets are changing with time. In the context of games, people want to experience playing and also break out of the story and venture within the game environment freely, changing and manipulating it to make it uniquely theirs. In the context of housing, especially in developed cities where a shortage of housing is an issue, the desire of the people is no longer to simply 'own a house' but to 'own a personalised house' where they can design a living space that fits their needs and share their creativity with their community. This desire can be observed in the participants who tried out the design process through the digital system. They demonstrated great enthusiasm and wanted more from the system. We believe that the present is the ideal moment to introduce just such a design system for homebuyers to contribute to a more sharing environment among them.

\subsubsection{TOWARDS DESIGN 3.0}

John Markoff (2006) of the New York Times named the current third-generation web, 'Web 3.0'. In other words, he is suggesting that the web is growing collectively towards 'the Intelligent Web' which includes services such as data-mining and machine learning to provide a more productive and intuitive user experience (Markoff, 2006). If we refer to the definition of Web 1.0 and Web 2.0 (O'Reilly, 2005), Design 1.0 can be defined as a design generated solely by the architect and Design 2.0 as similar to BIM, where a team shares information to produce a design. With technology fast advancing, design is becoming more connected, open and 
intelligent, and these factors are what define Design 3.0. Design brands such as Nike are opening design opportunities to consumers; scientists and artists alike are connecting with the public to develop ideas and solutions collectively, and generative systems and algorithms are being developed to automate design solutions. There is a high possibility that a 'Design 3.0' is emerging. We observed through interactions with the research participants and the collaboration process that the third order of synergy, generating new knowledge through shared information, is very much possible. It could be just a matter of time before Design 3.0 becomes a reality and incorporating homebuyers in the mass housing design process becomes a trend.

\subsection{Summary}

We are certain, from the study of this research, that engaging homebuyers collectively can change the current housing design implementation process. With today's easy access to the network, we believe that a digital tool, with the proper motivational forces, will significantly change the paradigm of the housing design collaboration process. In general, paradigms are complex, self-sustaining structures maintained by established systems that are part of the prevailing social, cultural, economic, aesthetic, psychological, technological and linguistic environment (Wood, 2016). These paradigms have factors that are so tightly knitted together it is hard to change them unless they can be addressed in a comprehensive manner. It is necessary to identify the points of intervention, using an extensive and radical method. We use gamification as a method to understand the factors that are necessary to immerse homebuyers in the housing design process, allowing us to achieve the research aim and develop the design decision support framework more effectively. The collaborative framework established in this research achieves its aim of playing the role of a spark, in hopes of setting off a series of further discussions that could drive changes to the housing design paradigm. 


\section{Chapter 8: Limitations of the Study}

Luxury to have a house and more luxury to talk about the house before it is built.

- John Habraken -

(2013)

This body of research explores how a transparent design environment that allows nonprofessionals the opportunity to participate in the design process can be produced. Digital platforms allow non-professionals (homebuyers in this context) to express their needs and desires for their living space, and collaborate with architects to achieve them. However, in light of the current housing market, many people question the cost aspect of this research: "How will this design method increase the possibility of getting a house if a house is still so expensive?" Even Habraken, after documenting his ideas and theories, ended with the quote that housing is a 'luxury'. The answer to that question in this research is 'undefined'. Cost is not such an important consideration with respect to the nature of this research. It is just another 'parameter' that can be easily implemented in the design environment. Although it would be ideal if the outcome of this research lessened the burden of getting a house, this is not what this research is aiming for. The challenge of this research is for homebuyers, having accumulated a huge amount of money through savings or loans, to achieve what they need instead of a typical prefabricated unit. By excluding the cost, or minimising its significance, homebuyers focus on 'design' and not 'value management'. More importantly, this research commenced with achieving a 'dream'. A dream of not only owning a home, but designing a home specifically for the homebuyer.

The aim of this chapter is to explicitly indicate the limitations of this study and explain how, even if excluded, they still influence this research significantly. 


\subsection{FIGURATIVE COST OF HOUSING}

Since the research focus is on the homebuyers, some details are not implemented in the design system, resulting in its limitations. This includes the exact cost of the housing. The research does not want to touch the realm of the housing market as this additional variable will cause the research to lose its focus. Currently, the cost of the housing used in the experimental studies are figurative numbers that are proportional to the budget allocated to role that the participants played. This is similar to the board game Monopoly, where the land value given is based solely on the game itself. Still, the participants were given a realistic experience by having the cost of the housing based on the average cost of land in their context (for example, the cost of the units' land is taken from the Global Property Guide as a general reference). Similarly, the integration of specific building standards would cause the scope of the research to become too big to handle. Only general building standards such as building core and structure are integrated as a foundation. This is based on the 'support' described in Open Building Concept (Kendall S. , 2004). However, these two aspect of housing design definitely require consideration in future research.

\subsection{STATUS OF PARTICIPANTS}

Putting the limited number of participants aside (this is explained in a previous chapter), another limitation of the participants is that they are not real homebuyers. As stated in previous chapters, they are either designers who are still in training, professionals in practice or simply the public who are just trying out the system by role-playing as homebuyers. However, they all have the desire to acquire a personal living space of their own that fits their specific needs. Most of the public participants also stated a desire to design their own living space. Role-play, as defined by Cohen and Manion (1980), is "participation in simulated social situations that are intended to throw light upon the role/rule contexts governing 'real' life social episodes". The key here is not 'who' are the participants but 'what are the mindsets' of the participants. Without real development, the context is all made-up, there is little value adding in using real homebuyers. The 'controlled environment' can be observed to loosen up as the studies 
continue till the third studies opening to the general public. This can be observed in the third study that the engagement process does not have much difference as compared to the studies using the students. In addition, by using focus group and role-playing, the researcher can engage closely with the participants to think of ways to improve the system together. This is close enough for this research to get the information necessary for evaluation.

\subsection{SUBJECTIVE OBSERVATION}

Coming to the evaluation, the feedback from the participants comes mainly though surveys. The remaining analysis is based on the observations of the researcher across the whole design process. The challenge here is to understand if the participants enjoy the process and whether that enjoyment is due to the gamification elements of the system. The survey does not ask directly about gamification as that will confuse participants and distract from the purpose of the experiments. Most of the observations result from the researcher interacting and conversing with the participants to understand if they are truly immersed in the design collaboration process.

\subsection{SERVER LIMITATION}

With respect to the digital tool, setting aside the limitation of the interface (resolved by linking with third party software, Fuzor), another limitation of the tool is its communication interface. The limited capacity of the ModRule server sees available storage being taken up mainly by the model data. As a result, the communication record could not be stored. Therefore, although the design interaction is within a digital environment, the communication among participants is still gathered face-to-face in a specific location (Figure 76). 


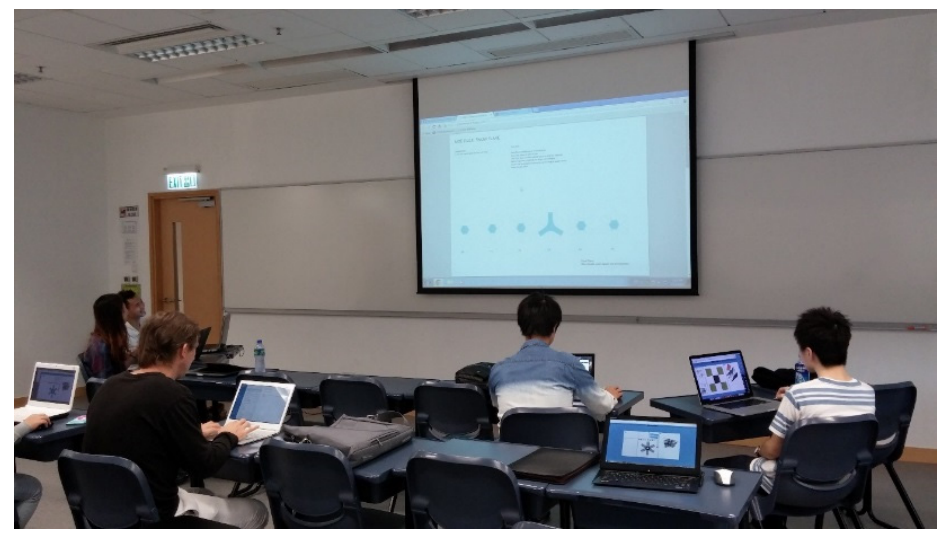

Figure 76 Participants engaging the digital tools, ModRule, within a location

The participants were eager to collaborate through the interface and the use of appropriate parameters enabled this process to proceed within the system. However, at some point, the simple chatbox interface became insufficient and participants ended up talking to each other for direct communication. This happens mainly when there is conflicting choices. Although the gamification techniques helped to mediate the design collaboration, there are still some experiments necessary in this aspect of the interface to test how the collaboration between homebuyers can be improved.

\subsection{TOUCHING ON GAME THEORY}

This leads to the next limitation of this research. The gamification techniques introduced in this thesis is at the level that is only sufficient to achieve the aim to understand the potential of digital platforms to promote design collaboration. In other words, more research is necessary to fully define the capacity of gamification within the context of housing design collaboration. Game theory alone is a huge topic, and this research has only touched the tip of the iceberg; using the most basic of its concepts to test the possibility of incorporating game theory into the design framework. Game theory is a powerful tool for a wide range of applications, including disaster relief (Nagurney, 2017). It is used mainly to understand the behaviour of competing decision-makers, which in this context are the homebuyers. However, the win-win theory discussed works only between two decision-making persons or parties, and only where there are just two choices. If the number of choices and the number of parties increases, the 
mathematics behind it changes (Levine, n.d.). The main point of introducing game theory is to present the possibility of quantifying these decision-making processes and to ensure that the parameters are not based only on the preferences of the architects, but calculated to provide optimal effect in the collaboration process. Understanding this approach, however, requires a whole new series of research.

This chapter summarises the challenges and limitations of this research, and how these are overcome and modified to fit the scope of this study. Although the research may look restricted as a result, the potential to branch out to future research is clearly demonstrated. The next chapter concludes the research and summarises the vision this research hopes to ultimately achieve. 


\section{Chapter 9: Conclusion}

You only express yourselves when you have a problem, when there are constraints. When you are totally free, you are lost.

- John Habraken -

(2013)

The completion of this thesis has been a challenging journey; one that has contributed new knowledge to collaborative housing design. Housing is an issue that touches everyone so easily encompasses a wide range of ideas. As a result, even defining the scope of the research is a challenge. The research cannot touch on too many issues, yet not neglect so much that the research is impractical. Many positive comments and criticisms arose along the way and what began as a personal indulgence has transformed through experimental studies into a critical body of research.

\subsection{Summary}

This research found that digital platforms complement the design process by bringing about closer relationships between homebuyers and architects through collaboration. To date, most research in collaborative design platforms focus primarily on generative mechanisms or algorithms to generate units that aim to suit homebuyers but may not. Most of the systems have hidden internal structures that limit the design control of the architects, ultimately approximating an ideal outcome for an 'imagined' homebuyer. Moreover, less attention is paid to enhancing the engagement of the homebuyers with the design process, especially the collaboration between individual homebuyers. As the research progresses, open building concepts and mass customizations emerge as possible solutions. However, this body of research argues that for apartment design to be more 'collectively productive', engagement between the homebuyers, and with architects, cannot be ignored. This research also identifies that digital platforms can provide fast and efficient means of communication, and that housing 
design involves many complex design components and operations that can be overwhelming for homebuyers.

This research, therefore, critically assesses digital interactions and design collaboration between homebuyers, and with the architect, using a digital platform. A novel digital platform was developed through two focus study groups and one large scale public engagement activity, which assessed the system on a different scale and context. The digital tool demonstrated a capacity to bring participants together to interact, engage, and develop a design, and achieve a unique result that can satisfy their needs. The studies conducted brought about better understanding, and so informed the development and specifications to further refine the collaboration process. In this way, the digital tool can be the main engagement channel for homebuyers and architects to collaborate on housing design.

Gamification concepts were introduced and integrated with the platform to explore and strategize the collaboration approach. Four factors were established as the key to greatly improve the design collaboration between homebuyers: adding motivational factors; simplifying the design process; formulating the decision network; enhancing communication in the digital platform. This research found that developing a collaboration technique of this type reinforces the role of architects in creating the built environment, by working closely with homebuyers to reflect their social needs.

A metadesign framework was developed based on the studies and evaluation of the digital tool. This framework affects the understanding and use of collaborative digital platforms in design. In particular, how to construct a design structure flexible enough for design intervention, how design options can be controlled, and which stage is optimal for participants to start engaging with the design. This also has implications for the development and evaluation of the collaboratively generated design outcome. More importantly, the third order of synergy emerging from the sharing of knowledge (in this case design knowledge) is fostered, along with a metadesign framework that can potentially be design 3.0 - making design more connected, open and intelligent. 


\subsection{Contributions}

This research makes four major contributions:

- Identification of requirements for a collaborative housing design process in digital platforms

The premise of this research is that collaboration with homebuyers is necessary to address their unique housing needs. This research has solidified that premise through the literature review and by identifying the weak elements of collaboration in the current mass housing design process. Most of the existing studies in similar subject areas focus on design generation and algorithms, so the perspective on homebuyers that this research takes may be used by future collaborative design developers to design an interaction process and to evaluate the functionality of new collaborative digital platforms.

- Formulating an engagement methodology for a collaborative design process Although the long-term benefits of a bottom-up, collaborative design process are known, they are not yet clearly distinguished and defined. The complexities and uncertainties of such design processes contribute to the current lack of confidence in adopting them. This research therefore provides a more obvious, tangible, immediate advantage at every stage of the design process. It not only produces a better understanding of using digital tools as a medium for collaboration; it demonstrates how such a design process can use gamification methods to create the necessary synergies to progressively bring about change to the housing system. With a virtual environment as an open design tool, a third order of synergy, at least, can be achieved; enabling the sharing of information and knowledge necessary to bring about a community of homebuyers even during the pre-occupancy stage.

\section{- Implementation and examination of a novel collaborative system tool}

A further contribution of this research is that a novel tool, ModRule, is tested to understand the engagement of laypersons with the design process of mass housing design. The capability of ModRule is brought about using BIM as background support and gamification as the driving factor for participation. ModRule has the potential in this context to amass diverse points of view and generate a great amount of creativity. This bottom-up and participatory approach responds to the needs of both architects and homebuyers and act as a medium for the collaboration between homebuyers. It proves effective, collaborative design possible with the help of computational tools not yet available.

\section{- Development of a collaborative design framework and culture}

The metadesign introduced through this research does more than provide a reference to guide the development of future digital tools. It represents a cultural shift in housing design. By promoting collaborative and transformational practices of design, to enable homebuyers to co-design their living space, this research hopes to support a new mode of human interaction, and sustain an expansion of creativity in the process. The metadesign here is therefore developing new ways of understanding and planning to produce more open and evolving systems of interaction. This also means that this research can be regarded not only as design approach, but also a form of cultural integration. 


\subsection{Future Directions}

This research is the first effort to examine collaborative design among homebuyers.

There several new research directions that can extend from the work of this research:

- Extension of the collaborative design tool to incorporate other stakeholders

In housing design, solutions result from the collaboration of professional stakeholders such as developers, contractors, engineers, surveyors, managers and architects. Homebuyers may present their needs and desires, but it is the professionals who realise the projects with respect to the urban environment, cost and building codes. The collaborative design engagement tool provides additional information that may help the architects and other professionals to better understand the housing needs, to review their design decisions, or to bring themselves closer to the homebuyers. This information is the key to deriving a higher order of synergy in the apartment building design context. Although the tool developed in this research does not support the connection between the homebuyers and professionals other than architects, it does provide a place holder for them. That is, the professionals are still considered an intrinsic component for the collaborative design process. From this perspective, the digital design tool can be extended to incorporate additional design capabilities to connect to other professionals.

\section{- Digital tool visualisation}

The focus group studies in this research indicate that visualization of the virtual design space is desirable. In this research study, third-party software is connected to test the use of a 3D digital environment and virtual reality in the design collaboration process. However, they are limited by software designed for professionals, not lay-homebuyers. Further studies are necessary to discover better visualization methods to allow homebuyers to more effectively communicate with each other, and with architects, within the design tool.

\section{- Motivational behaviour of gamification in the design process}

Behavioural studies are important to identify the behaviour patterns of homebuyers engaging with the design process. As demonstrated in the focus group studies, observing the participants provides important insights into the support required to facilitate the design interaction. This research examines gamification as one possible method and there are still many aspects of this to explore further. Other methods can also be explored to examine different motivational behaviour and evaluate collaborative design support.

\section{- Further investigation of collaborative design}

Workshops are conducted and presented (Lo et al., 2016, Lo, 2016) to demonstrate the potential of this research in participatory design even outside the realm of mass housing. The topics this research touches on can diverge to many other spheres of research. Mass housing design is just one area that could engage more with participants in the future, improving the lives of people and the built environment. 
This thesis ends with the unexpected result of a student exhibition that was not part of this research in any way. A group of building science students were tasked with building a façade to demonstrate their understanding of construction details. The physical model was collectively placed together to show a rich and dynamic outcome in an exhibition. If one takes a closer at the outcome (Figure 78), this is exactly the kind of exciting outcome that this research is aiming for. Instead of the homogenous and monotonous housing apartments that are filling up the urban spaces in cities, such a dynamic outcome could become the vertical version of Row Housing West 8 in Borneo Sporenburg, Amsterdam (Figure 77).

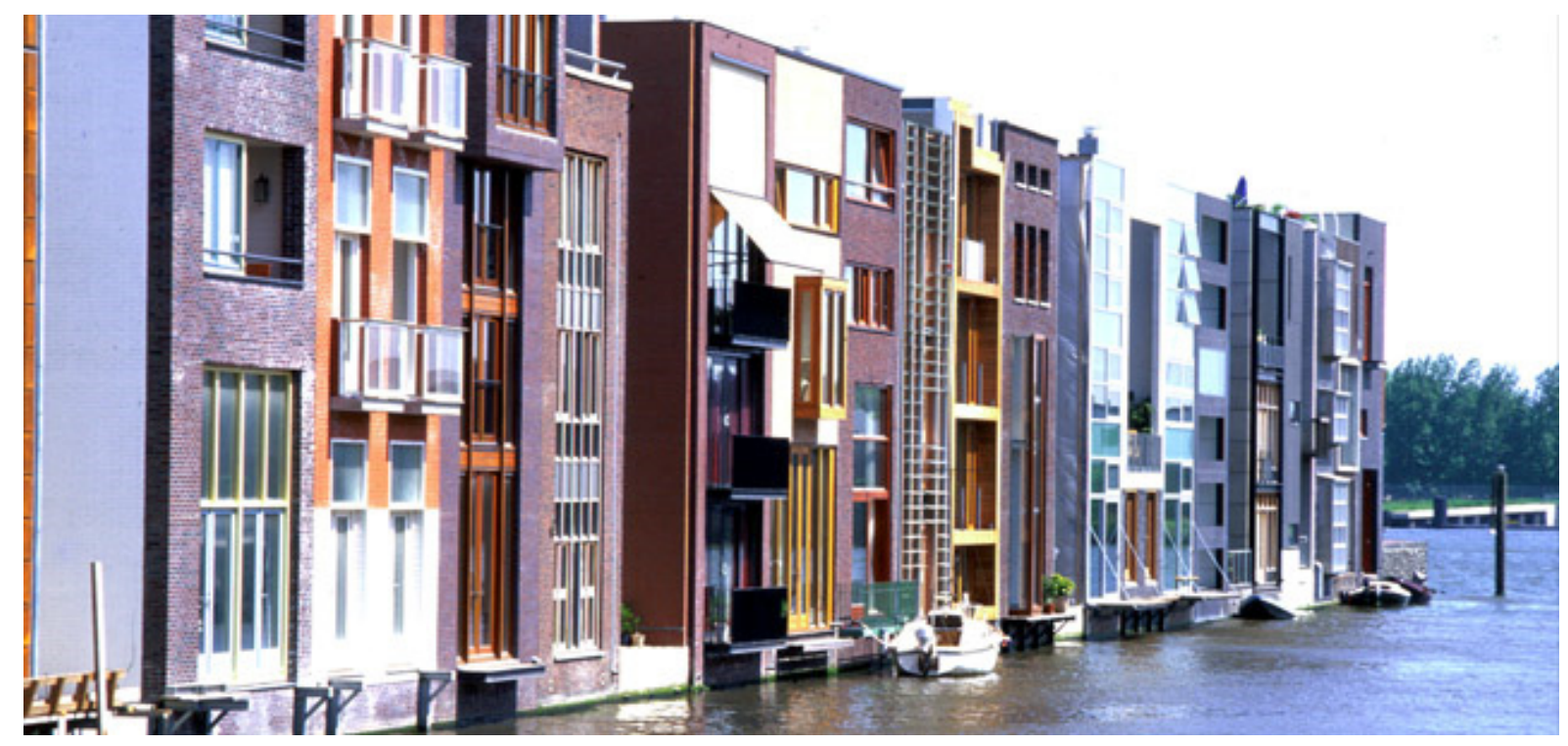

Figure 77 Row Housing West 8 in Borneo Sporenburg, Amsterdam (West 8, n.d.) showing a rich design outcome that gives every housing unit a unique outlook 


\section{Chapter 9 | Conclusion}

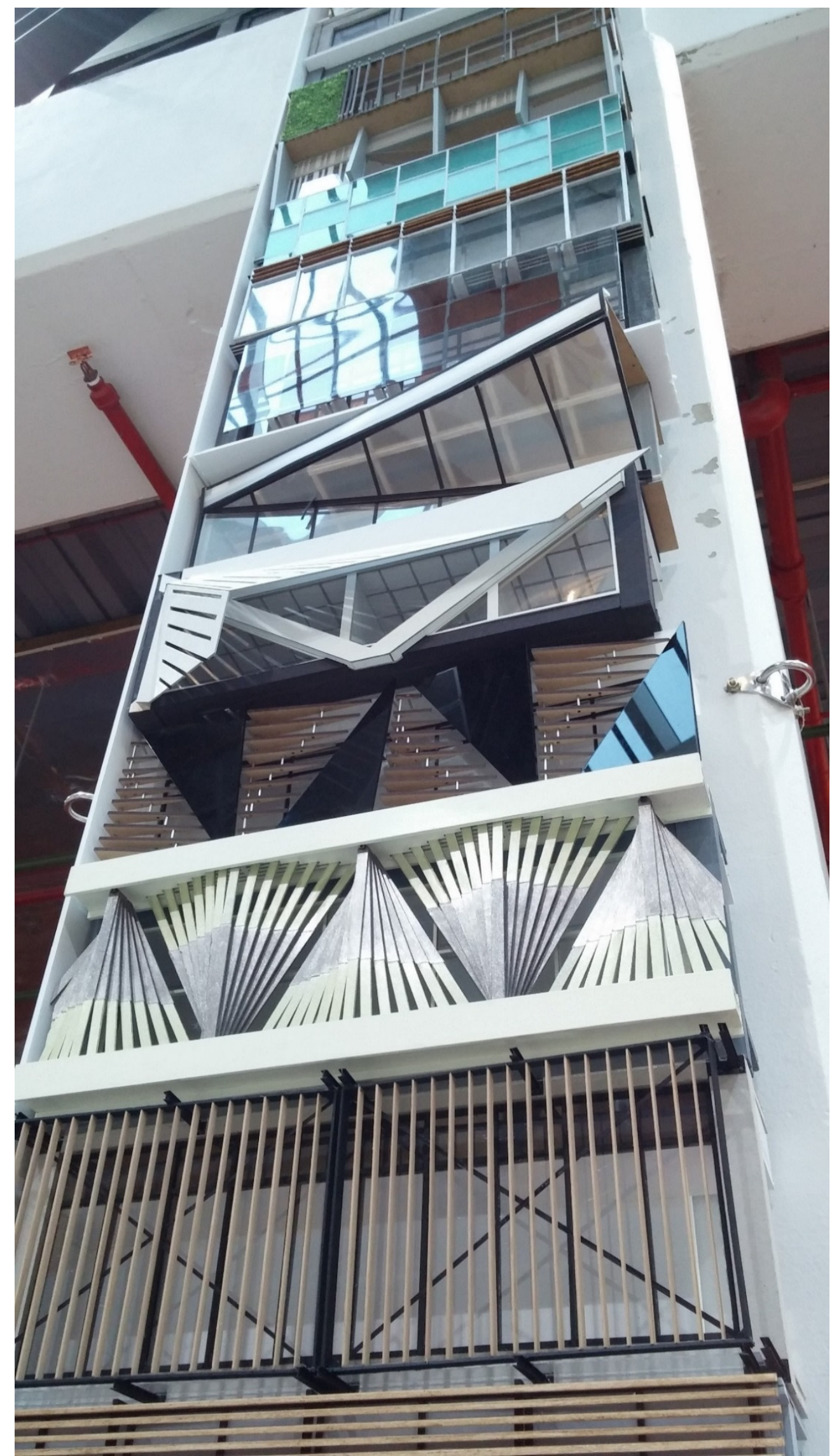

Figure 78 Collective façade design by a group of students from a building science design course. An unintentional outcome but a very desirable design solution that this research is hoping to achieve 


\section{References}

Alexander, C. (1964). Notes on the Synthesis of Form. Harvard University Press.

Alexander, C. (1975). The Oregon Experiment. New York: Oxford University Press.

Alexander, C. (2002). The Nature of Order: An Essay on the Art of Building and the Nature of the Universe. Berkeley: Center for Environmental Structure.

Alexander, C. (2006, Feb 3). To be a good builder, you need a feel for what surrounds you. Christopher Alexander knows. (K. Baker, Interviewer)

Alexander, C., Ishikawa, S., Silverstein, M., Jacobson, M., Fiksdahl-King, I., \& Angel, S. (1977). A Pattern Language: Towns, Buildings, Construction. New York: Oxford University Press.

alphr. (2015, Jan 9). What is Minecraft? We explore the global craze that's got both kids and adults hooked on virtual block-building. Retrieved from alphr:

http://www.alphr.com/games/1000235/what-is-minecraft-we-explore-the-global-crazethats-got-both-kids-and-adults-hooked-on

Andrews, K. (2008, Jan 17). BEDZED: Beddington Zero Energy Development in London. Retrieved from inhabitat: http://inhabitat.com/bedzed-beddington-zero-energydevelopment-london/

ARC Engineering and Architecture La Salle. (2014, July 31). Barcode Housing System. Retrieved from SlideShare: https://www.slideshare.net//madrazo/barcode-housingsystem

Arch+. (Oct 1989). Wohnung (die) für den Zweitmieter. Habitat (the) for the second tenant. The future of the Past. Arch+ (Deu) No.100-101, 30-45.

Asbury, J.-E. (2016). Overview of Focus Group Research . Qualitative Health Research Vol 5, Issue 4, 414 - 420.

ATD. (2012, Sept 14). 建筑设计作品 (ch) Architecture Design Project. Retrieved from 建筑技 艺-原建筑技术与设计 (ch) Architecture Technique:

http://www.atd.com.cn/ltem/3274.aspx\#Title

Axelrod, R., \& Dawkins, R. (1984). The Evolution of Cooperation. New York: Basic Books Inc.

Aydin, S., Lo , T., \& Schnabel, M. (2014). Gamification of Shape Grammars - Collaborative and Participatory Mass-Housing Design for Kashgar Old Town. Fusion - Proceedings of the 32nd eCAADe Conference - Volume 1 (pp. 603-612). England: Department of Architecture and Built Environment, Faculty of Engineering and Environment, Newcastle upon Tyne.

Beisi, J. (1995). Adaptable Housing For Adaptable People? Experience in Switzerland gives a new answer to the questions of adaptability. Architecture et Comportement / Architecture and Behaviour, 11(2), 139-162.

Benros, D., \& Duarte, J. (2009). An integrated system for providing mass customized housing. Automation in Construction Vol 18 (3), 310-320. 
Blecker, T., \& Friedrich, G. (2006). Mass Customization: challenges and solutions. New York: Springer.

Blenman, J. (2017, April 19). Adam Smith: The Father of Economics. Retrieved from Investopedia: http://www.investopedia.com/updates/adam-smith-economics/

Blum, B. (1996). Beyond Programming: To a New Era of Design. Oxford University Press.

BMC2. (2016). Vertical Corridor. Retrieved 2016, from New Riches: http://www.nouvellesrichesses.fr/en/projets/vertical-corridor/

Bogost, I. (2011, August 8). Gamification Is Bullshit! My Position Statement at the Wharton Gamification Symposium. Retrieved from lan Bogost Blog: http://www.bogost.com/blog/gamification_is_bullshit.shtml

Bogost, I. (2011, May 3). Persuasive Games: Exploitationware. Retrieved from Gamasutra: http://www.gamasutra.com/view/feature/6366/persuasive_games_exploitationware.ph $\mathrm{p}$

Bosselmann, P. (2008). Urban Transformation: Understanding City Form and Design. Island Press.

Brillembourg, A., \& Klumpner, H. (2012). Torre David: Informal Vertical Communities. Zurich: Lars Muller Publishers.

Caillois, R. (1960). Man and the Sacred. New York: Press of Glencoe.

Caillois, R. (1961). Man, Play and Games. New York: Press of Glencoe.

Caillois, R. (1964). The Mask of Medusa. New York: C.N. Potter.

Carey, M., \& Asbury, J.-E. (2016). Focus Group Research. Routledge.

Center for better living. (1982). Century Housing System coordination Guidebook. Japan.

Chang, K. (2015, May 24). Explaining a Cornerstone of Game Theory: John Nash's Equilibrium. Retrieved from The New York Times:

https://www.nytimes.com/2015/05/25/science/explaining-a-cornerstone-of-gametheory-john-nashs-equilibrium.html

Chen, R., \& Schnabel, M. (2011). Multi-touch - the future of design interaction. CAAD Futures, (pp. 557-572). Liege.

Chien, S.-F., \& Shih, S.-G. (2000). A Web Environment to Support User Participation in the Development of Apartment Buildings. Special Focus Symposium on WWW as the Framework for Collaboration, InterSymp 2000, (pp. 225-231). Baden-Baden, Germany.

Chien, S.-F., \& Shih, S.-G. (2001). Design through Information Filtering. A search driven approach for developing a layperson's CAAD environment. Proceedings of the Ninth International Conference on Computer Aided Architectural Design Futures, (pp. 103110 ). Eindhoven.

Chou, Y.-K. (2015). Actionable Gamification - Beyond Points, Badges, and Leaderboards. Octalysis Media.

Coonradt, C. (1984). The Game of Work. Gibbs Smith.

Corbusier, L. (1923). Towards a new Architecture. France. 
Cuperus, Y. (2001). An Introduction to Open Building. The 9th Annual Conference of the International Group of Lean Construction. Singapore.

Danielsen, C. (1996). De-signed Ecology. Design: Evolution, Cognition. DEcon '94 Symposium (pp. 3-11). Helsinki: Acta Polytechnica Scandinavica.

Deterding, S., Dixon, D., Khaled, R., \& Nacke, L. (2011). From Game Design Elements to Gamefulness: Defining "Gamification". Proceedings of the 15th International Academic MindTrek Conference, (pp. 9-15). Tampere.

Dijk, C. v. (2013, Jan 24). Unfinished business - designing for the unknown. Retrieved from Caro van Dijk Architectuur: http://www.carovandijk.nl/stories/unfinished-businessdesigning-for-the-unknown/

Duncan, S. (2011). Minecraft, beyond construction and survival. Well Played 1,1, 1-22.

Echo, L. (2014, Mar 29). Minecraft: The gaming craze that has a generation of youngsters enthralled. Retrieved from ECHO: http://www.liverpoolecho.co.uk/whats-on/familykids-news/minecraft-gaming-craze-generation-youngsters-6892684

Elmasry, M., \& Farid, E. (2007). User Participation and Mass Customization as Key Factors in the Future Residential Building. Em'body'ing Virtual Architecture: The Third International Conference of the Arab Society for Computer Aided Architectural Design (ASCAAD 2007), (pp. 327-342). Alexandria.

Fiamma, P. (2008). D.I.G.I.T.A.L. Defining Internal Goals In The Architectural Landscape. First International Conference on Critical Digital: What Matters(s)? (pp. 35-40). Cambridge: Harvard University Graduate School of Design.

Fiamma, P. (2013). From Designed Cognition to Built Architecture. 2nd International Conference on Innovations in Electrical and Civil Engineering, (pp. 101-105). Pattaya.

Fichelet, M., \& Fichelet, R. (1973). Evolutionary Housing (fr) Le logement évolutif. France: Délégation générale à la recherche scientifique et technique.

Forty, A. (2000). Words and Buildings: A Vocabulary of Modern Architecture. Thames \& Hudson.

Friedman, A. (2011). Decision Making For Flexibility in Housing. UK: The Urban International Press.

Friedman, Y. (2016, Jan 27). Interview with Von a Friedman: "Imagine, Having Improvised Volumes 'Floating' In Space, Like Balloons". (V. Belogolovsky, Interviewer)

Frohn, M., Rojas Toledo, M., Ong, L., \& Behrbohm, P. (2010). Hinterland Köln. Retrieved from YouTube: https://www.youtube.com/watch?v=ZAfw5HSzJto

Frost, P., \& Warren, P. (2000). Virtual reality used in a collaborative architectural design process. Information Visualization, (pp. 568-573). London.

Fuchs, M., Fizek, S., \& Ruffino, P. (2014). Rethinking Gamification. meson press eG.

Fuller, R. (1975). Synergetics: Explorations in the Geometry of Thinking. New York: Macmillian Publishing Co. Inc.

Gao, Y., Guo, X., Lo, T., \& Chang, Q. (2015). Integrated Open Source Design for Architecture in High-Density Housing Practice. Journal of Civil Engineering and Architecture 9, 1085-1098. 
Gellen, M. (2017). Accessory Apartments in Single-family Housing. Routledge.

Giaccardi, E. (2005). Metadesign as an Emergent Design Culture. Leonardo Vol. 38 No. 4, 342-349.

Global Housing Watch. (2017, May 3). Global House Price Index. Retrieved from International Monetary Fund: http://www.imf.org/external/research/housing/

Graaf, R. d. (2016, July 26). Few architects have embraced the idea of user participation; a new movement is needed. Retrieved 2016, from The Architectural Review: https://www.architectural-review.com/rethink/viewpoints/few-architects-haveembraced-the-idea-of-user-participation-a-new-movement-isneeded/10008549.article

Habraken, J. (1972). Supports: an alternative to mass housing. New York: Praeger.

Habraken, J. (1998). The Structure of the Ordinary: Form and Control in the Built Environment. MIT Press.

Habraken, J. (1999). Supports. London: Urban International press.

Habraken, J. (2000). Open Building; brief introduction. Retrieved from Habraken: http://www.habraken.com/html/introduction.htm

Habraken, J. (2001, January). Define and Let Go. (K. Havik, \& H. Teerds, Interviewers)

Habraken, J. (2002). The Uses of Levels. Open House International Vol. 27(2).

Hayes, S. (2014, Jun 24). PREFAB : Future or Failure? - Dissertation by Sam Hayes.

Retrieved from issuu: https://issuu.com/samhayes1/docs/sam_hayes_-_dissertation

HDB. (2016). Public Housing - A Singapore Icon. Retrieved from Housing \& Development Board: http://www.hdb.gov.sg/cs/infoweb/about-us/our-role/public-housing--asingapore-icon

HKHA. (2016). Prefabrication in Housing Blocks. Retrieved from Hong Kong Housing Authority: https://www.housingauthority.gov.hk/en/businesspartnerships/resources/prefabrication-in-housing-blocks/index.html

Huang, C.-h. (2007). A Choice Model of Mass Customized Modular Housing by Internet Aided Design. Joint Conference Proceedings of International Mass Customization Meeting 2007 (IMCM'07) and International Conference on Economic, Technical and Organizational Aspects of Product Configuration Systems (PETO'07), (pp. 61-75).

Huang, C.-h. (2007). Virtual Architect, Questionnaire Approach of Programming Modular Houses. SIGraDi 2007 - [Proceedings of the 11th Iberoamerican Congress of Digital Graphics], (pp. 310-314). México.

Huang, C.-h., \& Krawczyk, R. (2006). i_Prefab Home - Customizing Prefabricated Houses by Internet-Aided Design. Communicating Space(s) 24th eCAADe Conference Proceedings, (pp. 690-698). Greece.

Huang, C.-h., \& Krawczyk, R. (2007). A Choice Model of Consumer Participatory Design for Modular Houses. Predicting the Future [25th eCAADe Conference Proceedings, (pp. 679-686). Frankfurt .

Huang, C.-H., \& Krawczyk, R. (2007). Web Based BIM for Modular House Development: Query Approach in Consumer Participatory Design. The Third International 
Conference of the Arab Society for Computer Aided Architectural Design (ASCAAD 2007), (pp. 559-570). Alexandria.

Huizinga, J. (1949 / 1938). Homo Ludens: A Study of the Play-Element in Culture. London: Routledge and Kegan Paul.

Israel, N. (2012, Jun). La civdad viva. Retrieved Jun 2014, from http://www.laciudadviva.org/blogs/?p=14164

Jakubowski, M. (2016, June 29). Open Building Institute: Eco-Building Toolkit. Retrieved from Kickstarter: https://www.kickstarter.com/projects/622508883/open-buildinginstitute-eco-building-toolkit/description

Kalloc. (2014). Fuzor. Retrieved from Fuzor: https://www.kalloctech.com/

Kapp, K. (2012). The Gamification of Learning and Instruction: Game-based Methods and Strategies for Training and Education. San Francisco: John Wiley \& Sons.

Karlgren, J., Höök, K., Lantz, A., Palme, J., \& Pargman, D. (1994). The glass box user model for filtering. 4th International Coriference on User Modeling. Hyannis: ACM.

Kelly, A. (2003). Decision Making Using Game Theory :An Introduction for Managers. Cambridge: Cambridge University Press.

Kendall, S. (2004). Open Building Concepts. Retrieved from Open Building: http://www.openbuilding.org/ob/concepts.html

Kendall, S., \& Teicher, J. (2000). Residential Open Building. Taylor \& Francis.

Kieran, S., \& Timbrelake, J. (2004). Refabricating Architecture: How Manufacturing Methodologies are Poised to Tranform Building Construction. New York: McGraw Hill.

Kim, J.-J., Brouwer, R., \& Kearney, J. (2002). NEXT 21: A Prototype Multi-Family Housing Complex. US: University of Michigan. Retrieved 2016, from http://www.umich.edu/ nppcpub/resources/compendia/ARCHpdfs/NEXT21.pdf

Krantz, B. (1976). The Swedish Experience. Centre Scientifique et Technique du Bâtiment, CSTB Paper No. 167.

Kroll, L. (1987). The Architecture of Complexity. Cambridge: MIT Press .

Kroll, L. (2013). Friendly Architecture. What is Good Architecture? OASE (90), 28-31.

Labarre, S. (2011, Aug 25). WikiHouse, An Online Building Kit, Shows How To Make A House In 24 Hours. Retrieved from Co.Design:

https://www.fastcodesign.com/1664873/wikihouse-an-online-building-kit-shows-howto-make-a-house-in-24-hours

Lastowka, G. (2011, Oct 5). Minecraft as Web 2.0: Amateur Creativity \& Digital Games. Retrieved from SSRN: https://ssrn.com/abstract=1939241 or http://dx.doi.org/10.2139/ssrn.1939241

Leighninger, M. (2006). The Next Form of Democracy: How Expert Rule Is Giving Way to Shared Governance and Why Politics Will Never Be the Same. Nashville: Vanderbilt University Press.

Lendt, B. (Director). (2012). Dreaming of a Tree House: Frei Otto's Ecological Project in Berlin [Motion Picture]. 
Lendt, B. (Director). (2015). NEXT21 - an experiment [Motion Picture].

Levine, D. (n.d.). What is Game Theory? Retrieved from Economic and Game Theory: http://levine.sscnet.ucla.edu/general/whatis.htm

Lo, T., Schnabel, M., \& Moleta, T. (2015). A User-Centric Mass Housing Design Platform. In G. Celani, D. Sperling, \& J. Franco, Computer-Aided Architectural Design Future. The Next City - New Technologies and the Future of the Built Environment: 16th International Conference, CAAD Futures (pp. 236-254). Sao Paulo: Springer.

Lüthi, S., \& Schwarz, M. (Directors). (2013). De Drager [Motion Picture].

Luthi, S., \& Schwarz, M. (Directors). (2013). DE DRAGER / A film about Architect John Habraken [Motion Picture].

Madrazo, L., Cojo, A., Sicilia, Á., \& Costa, G. (2010). Barcode Housing System: Applying ICT to Open Building and Mass Housing. 16th Open and Sustainable Building, (pp. 275288). Labein.

Madrazo, L., Rivera, O., Costa, G., \& Sicilia, Á. (2010). Barcode Housing System: Enabling user participation in housing design and construction. Collaborative Working Environments for Architectural Design.

Madrazo, L., Sicilia, Á., Mar, G., \& Angel Martin, C. (2009). Barcode housing system: Integrating floor plan layout generation processes within an open and collaborative system to design and build customized housing. Joining Languages, Cultures and Visions: CAADFutures, (pp. 656-670). PUM.

Maki, F. (1965). Some thoughts on collective form. In G. Kepes, Structure in Art and in Science (pp. 116-127). George Braziller.

Markoff, J. (2006, Nov 12). Entrepreneurs See a Web Guided by Common Sense. Retrieved from New York Times: http://www.nytimes.com/2006/11/12/business/12web.html

Maurios, G., \& Herrou, M. (1976). les Marelles, une structure servante irriguée de fluides (fr) Les Marelles, an irrigated serving structure of fluids. Cahiers du CSTB , No.167.

Mcdonald, C. (2007). Ephemera and the Architecture of National Identity: Symbolic Urban Spaces during the first Royal visits to New Zealand. Asia-Pacific Modernities Colloquium (p. ). Melbourne: Faculty of Architecture, Building and Planning, University of Melbourne.

McGonigal, J. (2010, Feb). Gaming can make a better world. Retrieved from TED: https://www.ted.com/talks/jane_mcgonigal_gaming_can_make_a_better_world

McGonigal, J. (2012). Reality is Broken: Why Games Make Us Better and How They Can Change the World. New York: Vintage.

McMeel, D. (2015, Oct 1). Savings from going digital. Retrieved from Build: https://www.buildmagazine.org.nz/articles/show/savings-from-going-digital/

Mens, J., \& Vreeswikj, K. (2016). Open Source Developing, Designing and Constructing Small Urban Areas with Re-used Demolition Waste in the city of Utrecht. SBE16 Sustainable Built Environment - Transition ZERO. Utrecht.

Merin, G. (2013, July 21). AD Classics: Habitat 67 / Safdie Architects. Retrieved 2016, from ArchDaily: http://www.archdaily.com/404803/ad-classics-habitat-67-moshe-safdie 
MetroQuest. (2015). MetroQuest: Engagement... optimized. Retrieved 2017, from http://metroquest.com/

Minami, K. (2016). The Efforts to Develop Longer Life Housing with Adaptability in Japan. Energy Procedia Vol. 96, 662-673.

Mohammadi, D. (2014, Jan 25). How online gamers are solving science's biggest problems. Retrieved from theguardian: https://www.theguardian.com/technology/2014/jan/25/online-gamers-solvingsciences-biggest-problems

Mufson, B. (2014, Apr 24). FutureCoast Turns Climate Science Fiction Into Potent Augmented Reality Experience. Retrieved from Creators: https://creators.vice.com/en_au/article/ez5dyp/futurecoast-turns-climate-sciencefiction-into-potent-augmented-reality-experience

Nabian, N., \& Ratti, C. (2012, March 2). A collaborative approach to architecture. Retrieved 2016, from MARKLIVES.COM:

http://senseable.mit.edu/papers/pdf/20120302_Nabian_Ratti_CollaborativeApproach_ Marklives.pdf

Nagurney, A. (2017, Sep 1). Time for some game theory: How responses to natural disasters like Harvey could be improved. Retrieved from Salon:

http://www.salon.com/2017/08/31/response-to-natural-disasters-like-harvey-could-behelped-with-game-theory_partner/

Nasar, J., \& Kang, J. (1999). House style preference and meanings across taste cultures. Landscape and Urban Planning 44, 33-42.

Nations, U. (2014). World Urbanization Prospects: The 2014 Revision, Highlights. United Nations: Department of Economic and Social Affairs, Population Division.

OECD. (2016). OECD Social and Welfare Statistics. Retrieved from OECD: Better policies for better lives: http://www.oecd-ilibrary.org/social-issues-migration-health/data/oecdsocial-and-welfare-statistics/family-indicators-edition-2016_a6b32120-en

Ong, E. H. (2011). Group Forming. Retrieved from Vimeo: http://vimeo.com/26638743

Ong, E., Janssen, P., \& Lo, T. (2013). Group Forming: Negotiating Design Via Web-Based Interaction and Collaboration. Open Systems: Proceedings of the 18th International Conference on Computer-Aided Architectural Design Research in Asia (CAADRIA 2013), (pp. 271-280). Singapore.

Oprea, R., \& Sarpe, A. (2009). Online vs. offline vs. mixed participation for better govenmental policymaking. The International Conference on Economics and Administration, Faculty of Administration and Business. Romania: University of Bucharest.

O'Reilly, T. (2005, Sept 30). What Is Web 2.0: Design Patterns and Business Models for the Next Generation of Software. Retrieved from O'Reilly:

http://www.oreilly.com/pub/a/web2/archive/what-is-web-20.html

Orland-Barak, L., \& Maskit, D. (2017). Methodologies of Mediation in Professional Learning. Springer. 
Osborne, H. (2006, Oct 30). Stern report: the key points. Retrieved from theguardian: https://www.theguardian.com/politics/2006/oct/30/economy.uk

Otto, F. (2012). Dreaming of a Tree House. (B. Lendt, Interviewer)

Paiement, G. (2015, May 13). Habitat 67, Montreal's 'failed dream'. Retrieved 2016, from theguardian: https://www.theguardian.com/cities/2015/may/13/habitat-67-montrealexpo-moshe-safdie-history-cities-50-buildings-day-35

Pak, B., \& Verbeke, J. (2014). ICT-enabled Civic Empowerment and Participation: in Design, through Design. Proceedings of the 32nd eCAADe Conference - Volume 1, (pp. 8997). Newcastle .

Paris, M. d. (2014). The Mayor, I have an idea (fr) Madame la Maire, j'ai une idée. Retrieved 2017, from https://idee.paris.fr/

Parvin, A. (2012, Aug 2). Open Source WikiHouse Disrupts Traditional Design. (E. O'Neil, Interviewer)

Parvin, A. (2013). Architecture (and the other 99\%) Open-Source Architecture and the Design Commons. Architectural Design Vol.83 (6), 90-95.

Periáñez, M. (1972). Housing with adaptable plans of Montereau-Surville (fr) Les logements à plan adaptable de Montereau-Surville. Laboratoire d'Anthropologie Appliquée, Vol.2.

Periáñez, M. (2013, June). L'habitat évolutif : du mythe aux réalités (fr) Evolving habitat: From Myth to Realities. Retrieved from Manuel Periáñez: http://mpzga.free.fr/habevol/evolutif2013.html

Perry, A. (2016, Oct 4). This unreleased game was 'Minecraft' years before 'Minecraft' existed. Retrieved from Tech Insider: https://www.businessinsider.com.au/game-zerowas-minecraft-before-minecraft-2016-10? $r=U S \& I R=T$

Pine, B. (1993). Mass Customization: The New Frontier in Business Competition. Boston: Harvard Business School Press.

Pollit, K. (1981). A Need for Change in United Kingdom Housing Attitudes. In O. Ural, \& R. Krapfenbauer, Housing: The Impact of Economy and Technology (pp. 159-171). Vienna: Elsevier.

Puusepp, R., Loke, T., \& Kivi, K. (2017). Enabling Customer Choice in Housing - Mass Customisation Solution for Prefabricated House Manufacturers. Protocols, Flows, and Glitches - Proceedings of the 22nd CAADRIA Conference (pp. 251-260). Suzhou: Xi'an Jiaotong-Liverpool University.

Rapoport, A. (1983). Pour une anthropologie de la maison (fr) For an anthropology of the house. Dunod.

Ratliff, S. (2017, May 2). The Changing Racial Demographics Around the World. Retrieved from Multiracial Media: http://multiracialmedia.com/changing-racial-demographicsaround-world/

Ratti, C., Antonelli, P., Bly, A., Dietrich, L., Grima, J., Hill, D., . . Sterling, B. (2011, June 15). Op-ed. Retrieved from domus: http://www.domusweb.it/en/op-ed/2011/06/15/opensource-architecture-osarc-.html 
Rogers, R. (2012, June 18). The Role of Architecture in Humanity's Story. (V. Shah, Interviewer)

Rougé, N. (2013, March 9). une autre ville. Retrieved 2016, from http://nicolasrouge.blogspot.co.nz/2013/03/verticalement-individuel.html

Rudofsky, B. (1964). Architecture Without Architects: A Short Introduction to Non-pedigreed Architecture. New York: Museum of Modem Art.

Salen, K., \& Zimmerman, E. (2003). Rules of Play: Game Design Fundamentals. MIT Press.

Sanchez, J. (2015). Block'hood - Developing an Architectural Simmulation Video Game. Real Time - Proceedings of the 33rd eCAADe Conference, (pp. 89-97). Vienna.

Schmidt, R., Eguchi, T., \& Austin, S. (2010). Lessons from Japan: A Look at Century Housing System. 12th International Dependancy and Structure Modelling Conference, (pp. 361-373). Cambridge.

Schmitt, G. (2015, Oct 26). L06 - Urban Science: Citizen Design Science. Retrieved 2017, from http://www.ia.arch.ethz.ch/l06-urban-science-citizen-design-science/

Schnabel, M. (2005). Interplay of Domains. Learning from the Past a Foundation for the Future [Special publication of papers presented at the CAAD futures 2005 conference, (pp. 11-20). Vienna.

Schnabel, M. (2006). Architectural Parametric Designing. Communicating Space(s) [24th eCAADe Conference Proceedings], (pp. 216-221). Volos.

Schnabel, M. (2007). Rethinking Urban Parameters. CAADRIA 2007 [Proceedings of the 12th International Conference on Computer Aided Architectural Design Research in Asia], (pp. 625-632). Nanjing.

Schnabel, M. (2009). MIXED REALITIES: Framing Mixed Realities. In X. Wang, \& M. Schnabel, Mixed Reality In Architecture, Design, And Construction (pp. 3-11).

Schnabel, M., \& Kvan, T. (2001). Design communication in immersive virtual environments: An initial exploration. Architectural Information Management [19th eCAADe Conference Proceedings], (pp. 472-478). Helsinki.

Schnabel, M., \& Kvan, T. (2001). Three-D Maze: Getting Lost in Virtual Reality. SIGraDi biobio2001 - [Proceedings of the 5th Iberoamerican Congress of Digital Graphics], (pp. 145-147). Concepcion.

Schnabel, M., \& Kvan, T. (2002). Immersive 3D architectural worlds: How to get in and out again. Connecting the Real and the Virtual - design e-ducation [20th eCAADe Conference Proceedings], (pp. 592-596). Warsaw.

Schnabel, M., \& Kvan, T. (2003). Spatial Understanding in Immersive Virtual Environments. International Journal of Architectural Computing vol. 1 - no. 4, 435-448.

Schnabel, M., \& Mercedes, P. (2008). Surfing The City: Towards context-aware mobile exploration. CAADRIA 2008 [Proceedings of the 13th International Conference on Computer Aided Architectural Design Research in Asia] , (pp. 383-390). Chiang Mai .

Schwartz, B. (2003). The Paradox of Choice: Why More is Less. HarperCollins.

Segard, A., Moloney, J., \& Moleta, T. (2013). OPEN COMMUNITITION: Competitive design in a collaborative virtual environment. Open Systems: Proceedings of the 18th 
International Conference on Computer-Aided Architectural Design Research in Asia, (pp. 231-240). Singapore.

Seiichi, F. (2008). The History of Developments toward Open Building in Japan. Education for an Open Architecture, 64-71.

Short, D. (2012). Teaching scientific concepts using a virtual world-Minecraft. Teaching Science-the Journal of the Australian Science Teachers Association, 55-58.

Sicilia, Á., Madrazo, L., \& González, M. (2011). Applying Clustering Techniques to Retrieve Housing Units from a Repository. Design Computing and Cognition '10 (pp. 387-401). Dordrecht: Springer.

Singh, B. (2010, Dec 22). What is the difference between Pilot and Beta testing? Retrieved from Software Engineering: http://soft-engineering.blogspot.co.nz/2010/12/what-isdifference-between-pilot-and.html

Skipsey, C. (2015, May 07). Top Down vs Bottom Up Architecture. Retrieved from When Wind Comes Alive: http://www.gwts.com.au/blog/entry/top-down-vs-bottom-uparchitecture

Smuts, H., Van der Merwe, A., Kotze, P., \& Loock, M. (2010). Critical Success Factors for Information Systems Outsourcing Management: A Software Development Lifecycle View. Proceedings of the SAICSIT 2010 Conference: Fountains of Computing Research, (pp. 304-313). New York.

sol•id•ar Architekten. (1999). "Ökohaus" Berlin. Retrieved 2016, from http://www.solidararchitekten.de/projekte/baugemeinschaft/solidar-oekohaus-berlin.html

Spatial Agency. (2011). Walter Segal. Retrieved from Spatial Agency: http://spatialagency.net/database/how/empowerment/walter.segal

Spatial Agency. (n.d.). John Habraken. Retrieved from Spatial Agency: http://www.spatialagency.net/database/john.habraken

Spivack, N. (2017). Web 3.0: The Third Generation Web is Coming. Retrieved from Lifeboat Foundation Safeguarding Humanity: https://lifeboat.com/ex/web.3.0

Stott, R. (2014, Sept 22). WikiHouse Unveils World's First Two-Storey Open-Source House at London Design Festival. Retrieved from archdaily:

http://www.archdaily.com/550093/wikihouse-unveils-world-s-first-two-storey-opensource-house-at-london-design-festival

Tafuri, M. (1976). Architecture and Utopia: Design and Capitalist Development. England: MIT Press.

Taniguchi, J. (2012). Space Planning of Well-being House (Chi translated from Jap). Taiwan: Yeren.

Tereci, A. (2017). Importance of Social Sustainability at the Mass Housing Projects. 3rd International Conference on New Trends in Architecture and Interior Design. Helsinki.

Till, J., \& Schneider, T. (2005). Flexible housing: the means to the end. Architectural Research Quarterly, 9 (3-4), pp. 287-296.

Toll Brothers. (n.d.). Toll Brothers. Retrieved from Design your own home: http://www.designyourownhome.com/ 
Tsutsui, K. (2010). 11 Koji Tsutsui. JA: The Japan Architect 77, 102.

Tutorialspoint. (2006). Software Design Strategies. Retrieved from Tutorialspoint: https://www.tutorialspoint.com/software_engineering/software_design_strategies.htm

Urban ThinkTank. (2015, April). Torre David. Retrieved from Urban ThinkTank: http://utt.com/project/torre-david/

Verba, S., Schlozman, K., \& Brady, H. (1995). Voice and Equality: Civic Voluntarism in American Politics. Cambridge: Harvard University Press.

Vulture, C. (2014, Aug 15). How Cultural Differences Impact Interior Design. Retrieved from Commisceo Global: http://www.commisceo-global.com/blog/how-cultural-differencesimpact-interior-design

West 8. (n.d.). BORNEO-SPORENBURG. Retrieved from West 8: http://www.west8.nl/projects/all/borneo_sporenburg/

Wilkinson, S. (1998). Focus group methodology: a review. International Journal of Social Research Methodology Volume 1, Issue 3, 181-203.

Wong, D. (2011, May 25). NikeID Makes \$100M+: Co-Creation Isn't Just a TrendNIKEiD. Retrieved from Huffpost: https://www.huffingtonpost.com/danny-wong/nikeid-makes100m-co-crea_b_652214.html

Wong, S., Lai, W., Ho, C., Chau, K., Lam, L., \& Ng, H. (2009). Sick building syndrome and perceived indoor environmental quality: A survey of apartment buildings in Hong Kong. Habitat International Vol.33 (4), 463-471.

Wood, J. (2007). Design for Micro-Utopias: Making the Unthinkable Possible. England: Gower.

Wood, J. (2016). Why Do We Need Metadesign? Retrieved from Metadesigners: https://metadesigners.org/Metadesign-Introduction

World Family Map. (2014). Family Structure. Bethesda: World Family Map.

Wright, R. (2001). Nonzero: The Logic of Human Destiny. Vintage.

Zeisel, J. (1981). Inquiry by Design: Tools of Environmental Behavior Research. Montreal: Cole Publishing Co.

Zichermann, G., \& Cunningham, C. (2011). Gamification by Design: Implementing Game Mechanics in Web and Mobile Apps. O'Reilly Media, Inc. 


\section{Appendices}

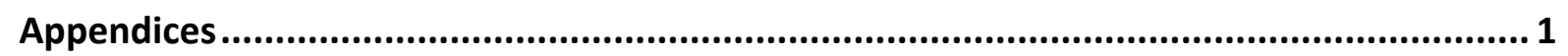

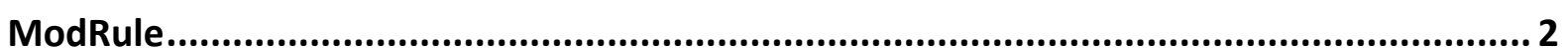

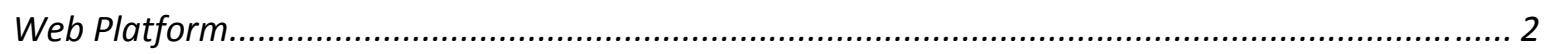

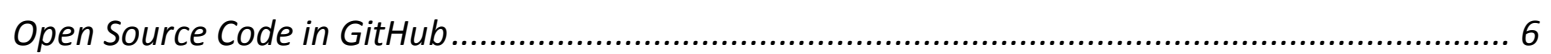

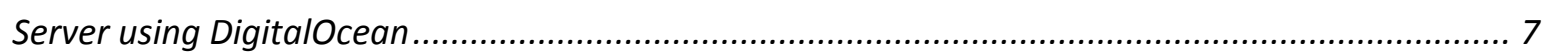

Pre-Development Focus Group Study ................................................................... 8

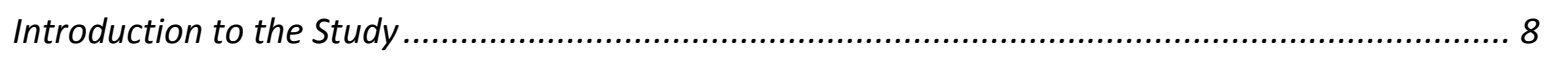

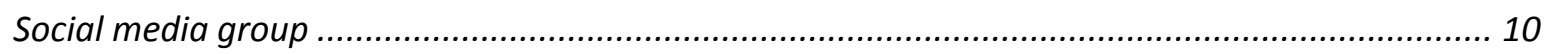

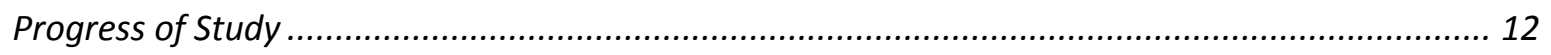

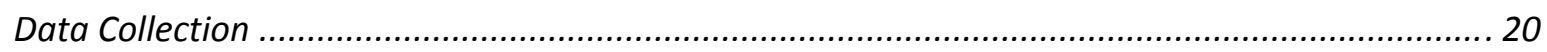

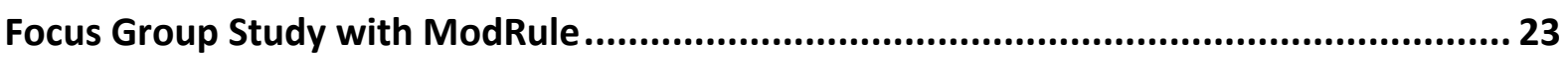

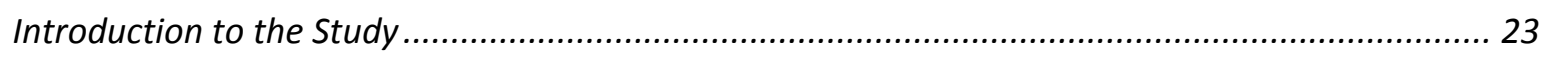

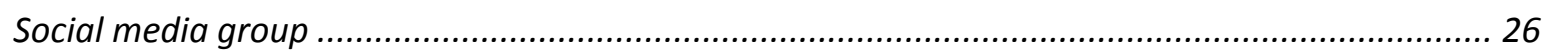

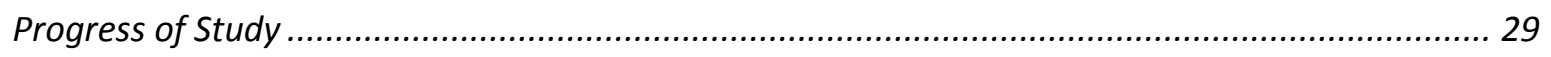

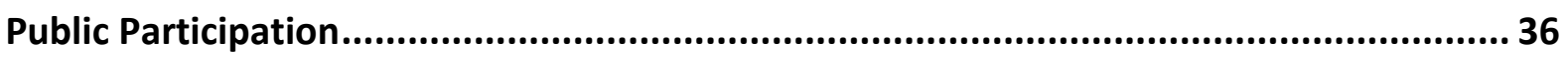

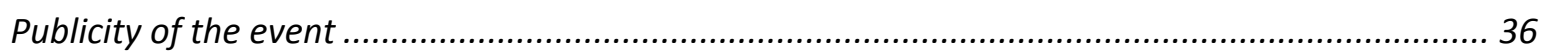

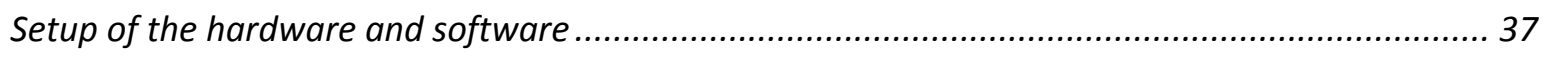

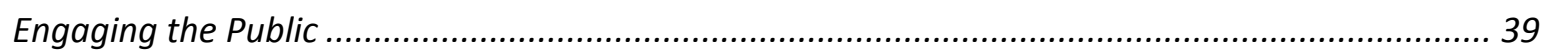

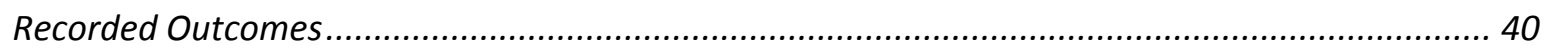

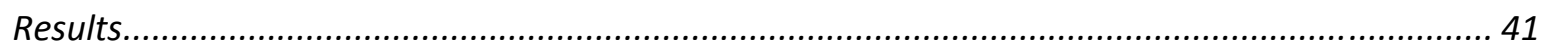

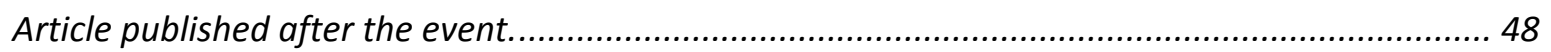




\section{ModRule}

ModRule is a web-based platform developed for the study of this research. This can be found at www.module.com. For examination purpose a sample login can be used with the following: username: SkyLott, password: s1k2y3 (both case sensitive). Below are screenshots of the interface.

\section{Web Platform}

1. Login page.

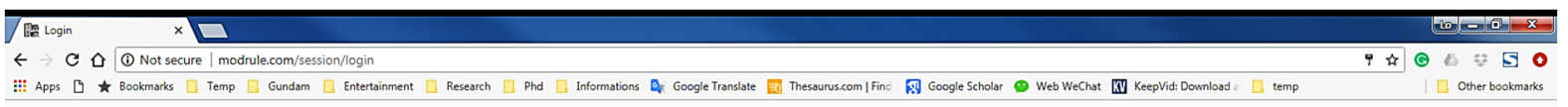

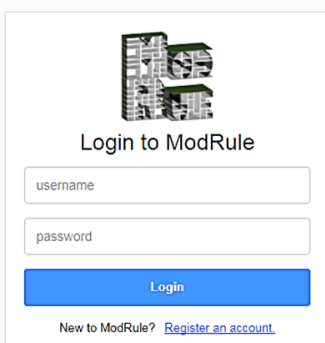

A PhD research by Shy Lo Tian Tian

Professor Marct Aurel Schnabel

0 2013-2017 DAPE

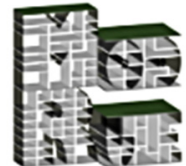

Login to ModRule

username

password

Login

New to ModRule? Register an account.

A PhD research by Sky Lo Tian Tian with

Professor Marc Aurel Schnabel

Lance Shi

Allen Xu

(c) 2013-2017 DARA 


\section{2. 'Create' interface.}

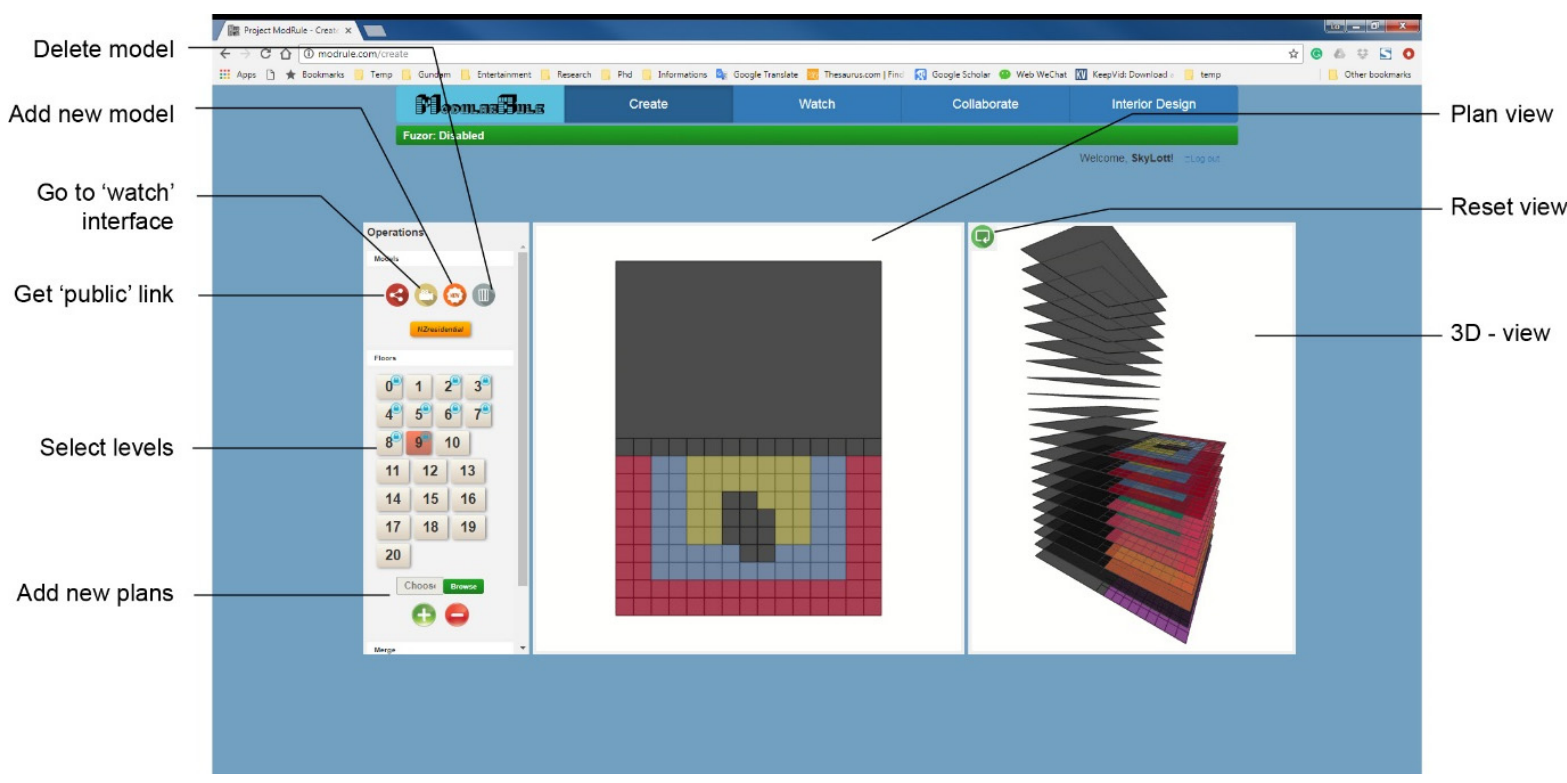

3. 'Create' interface with more 'operations'.

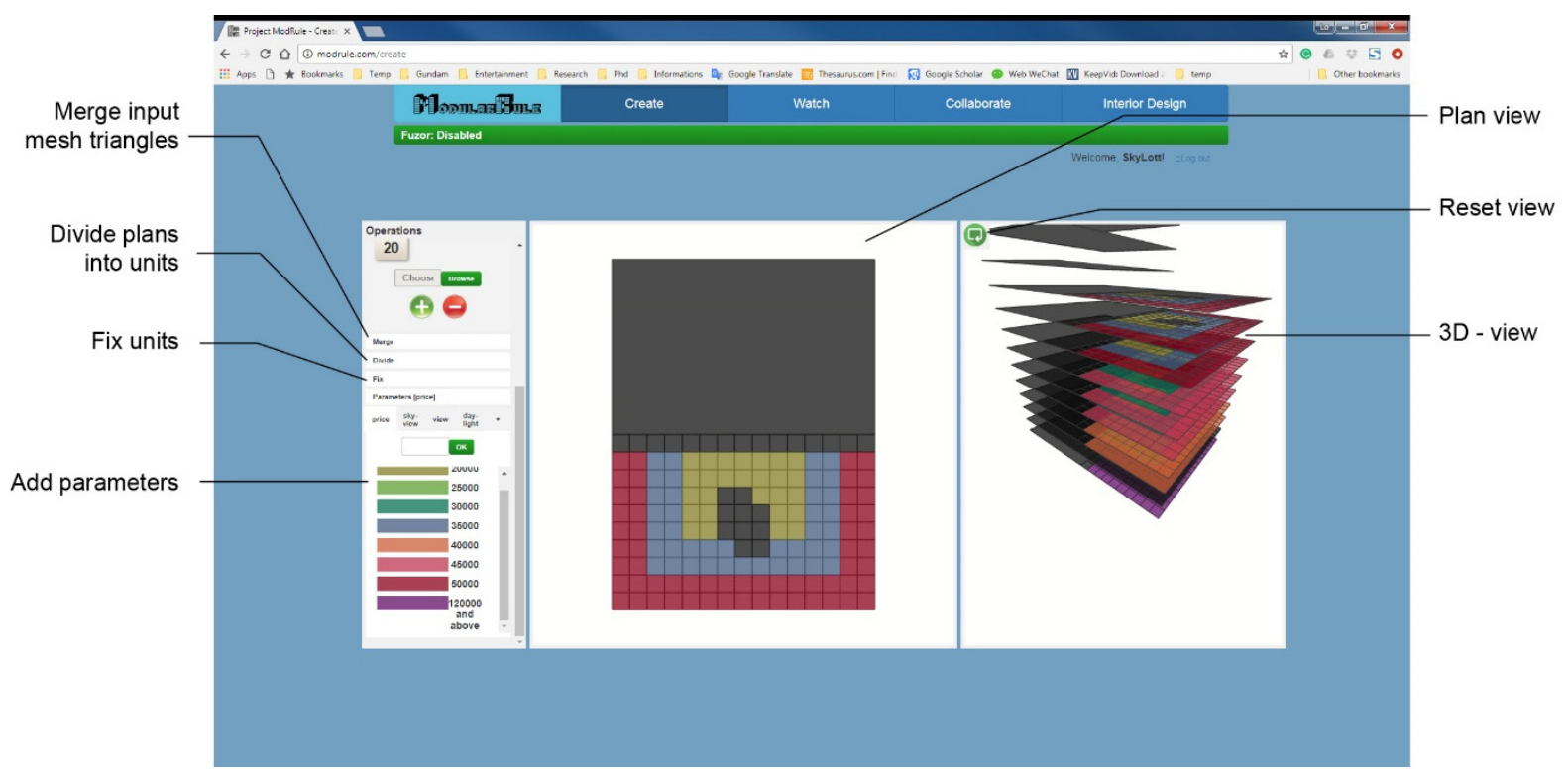




\section{4. 'Watch' Interface.}

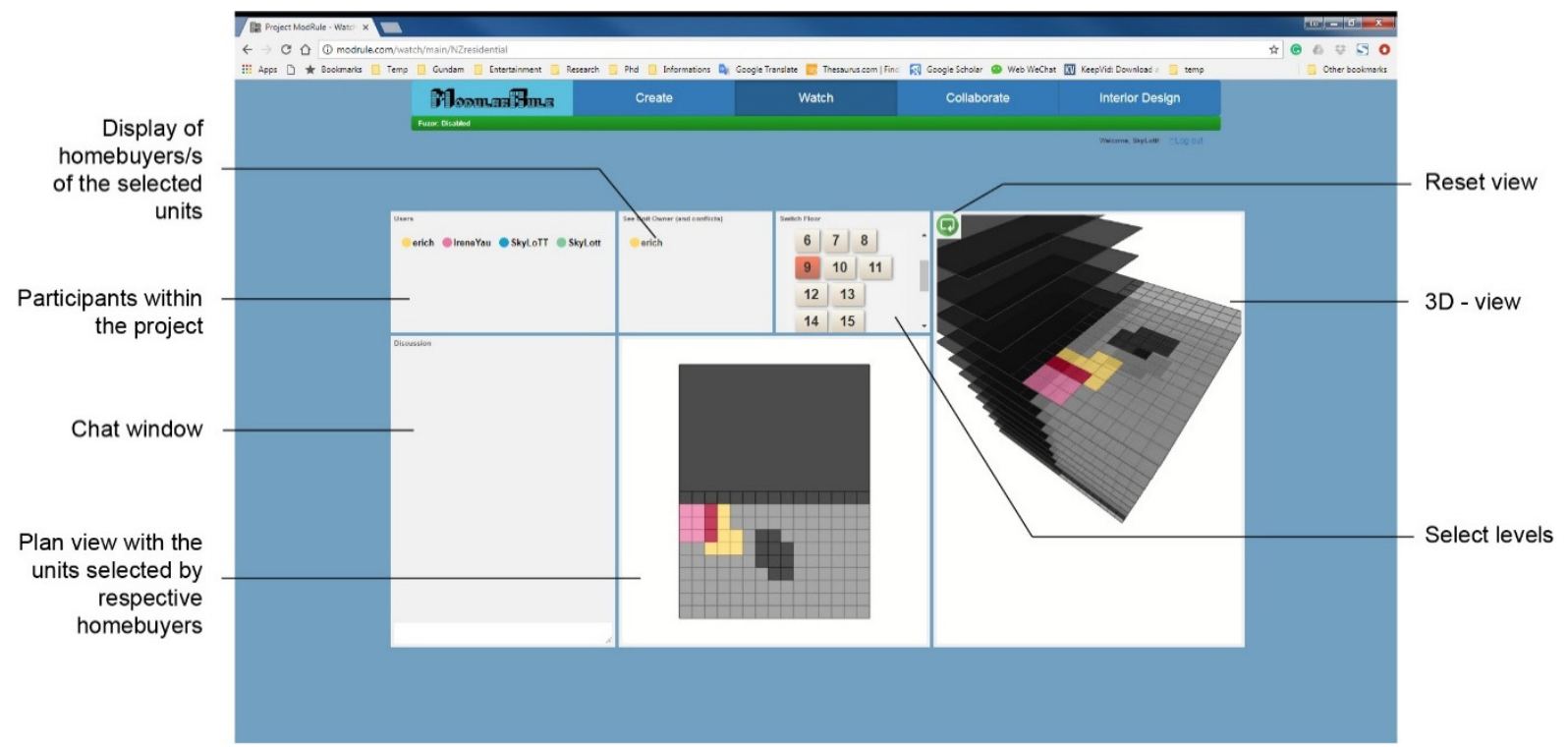

\section{5. 'Collaborate' interface.}

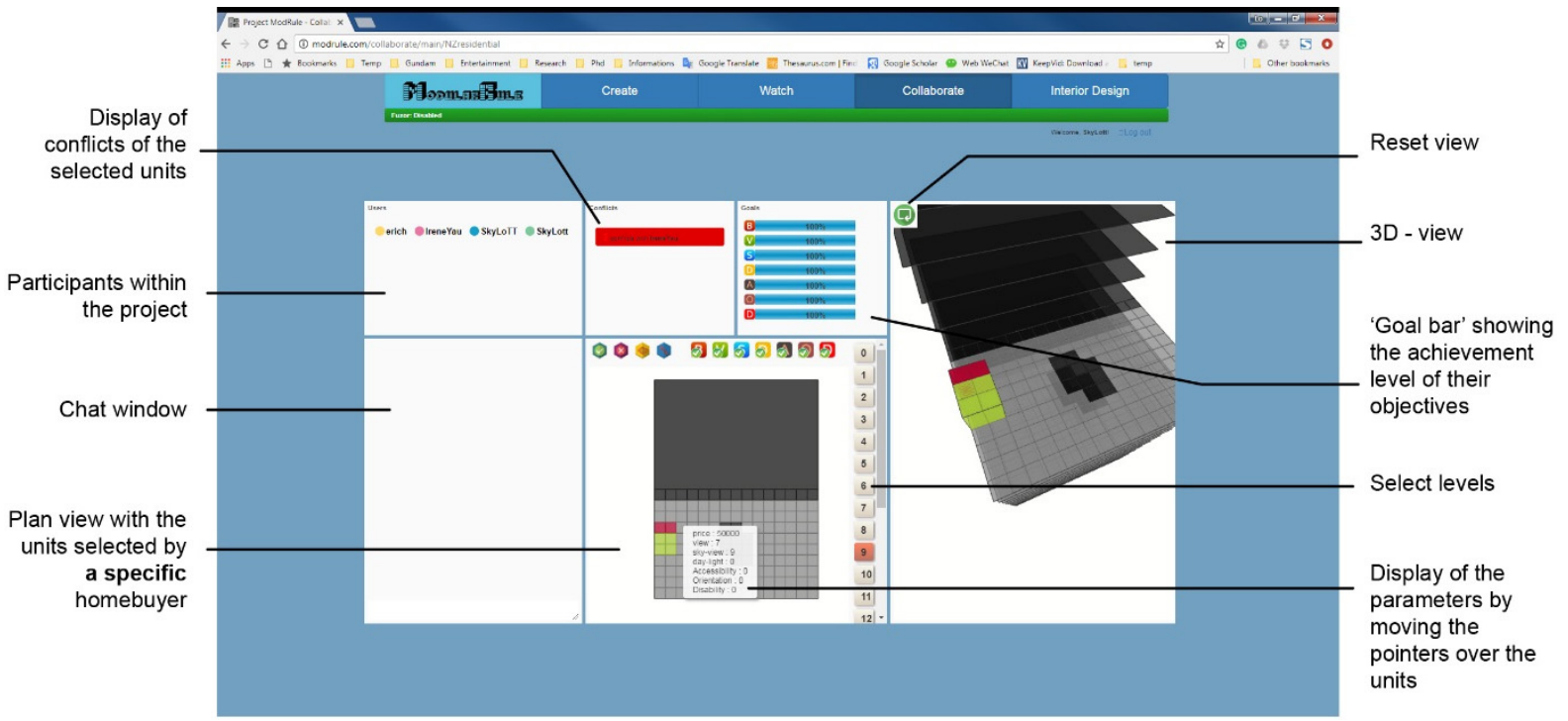


6. 'Interior Design' interface empty.

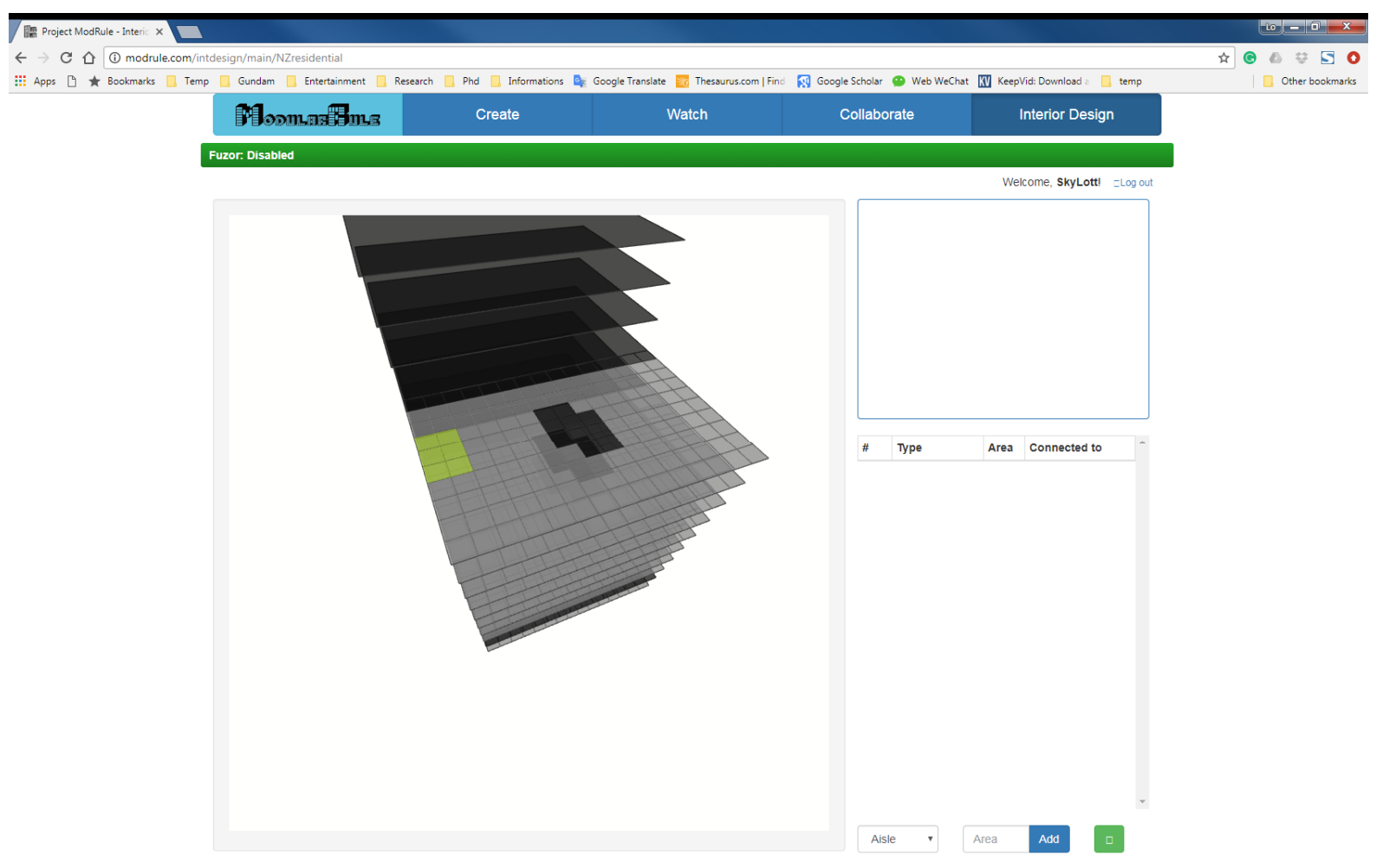

7. 'Interior Design' interface with user input showing intended connection and detail of space.

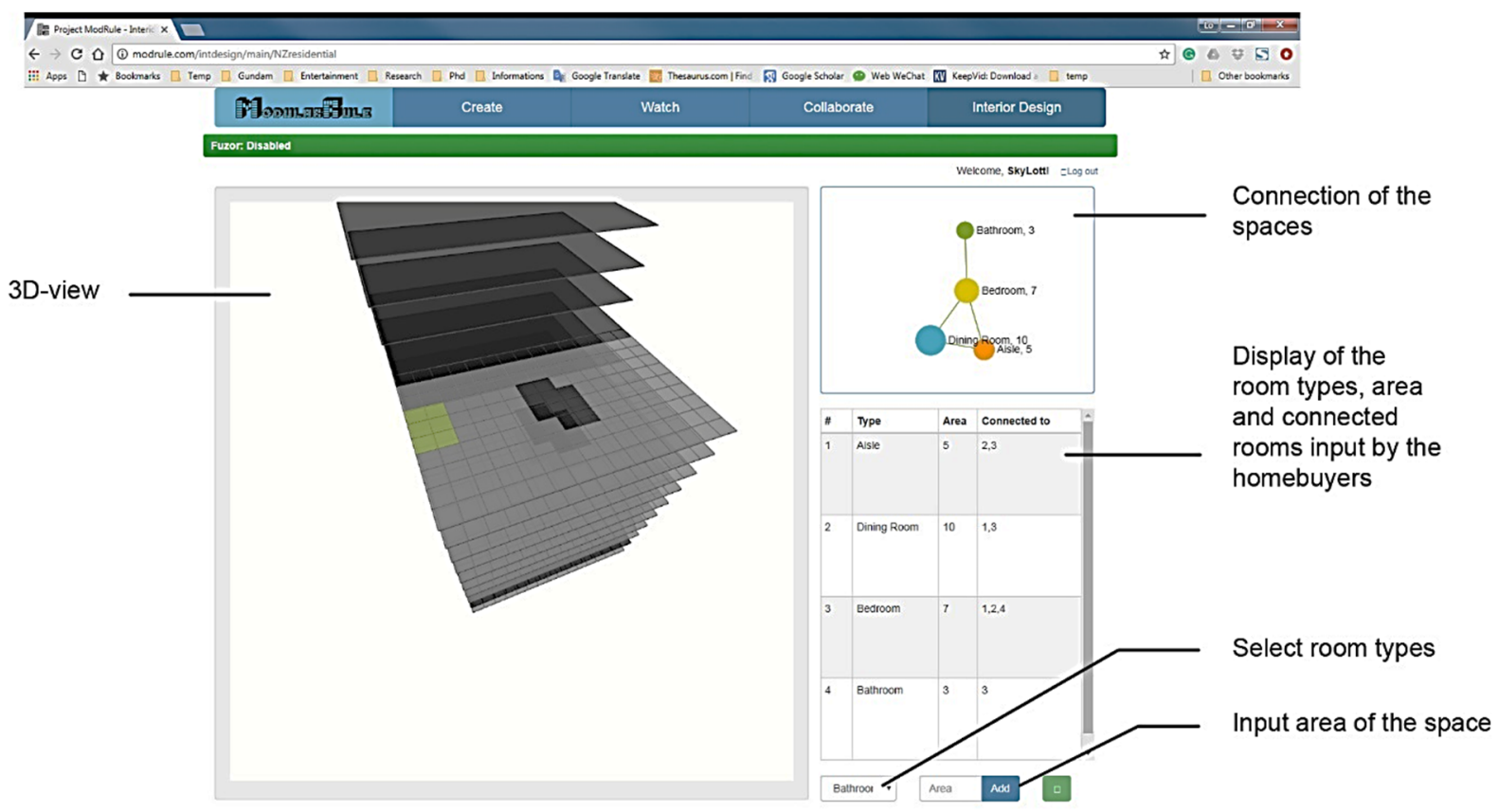




\section{Open Source Code in GitHub}

GitHub is a web platform for teams to work together in developing codes. The ModRule system developed in this research used GitHub to collaborate among other programmers. The collaborators in GitHub provided technical advices and help for this research to develop ModRule.

1. ModRule on GitHub showing its open source collaborative code structure.

\section{(5) This repository Search Pull requests Issues Marketplace Explore}

\begin{tabular}{|c|c|c|c|c|c|c|c|c|c|c|c|}
\hline B modrule & / collaborat & Private & & & & () Unwatch - & 8 & $\downarrow$ Star & 0 & 8 Fork & 0 \\
\hline 〈Code & (1) Issues 0 & I] Pull requests 0 & [II] Projects (0 & 国 Wiki & $\omega_{1}$ Insights & Settings & & & & & \\
\hline
\end{tabular}

No description, website, or topics provided.

Add topics

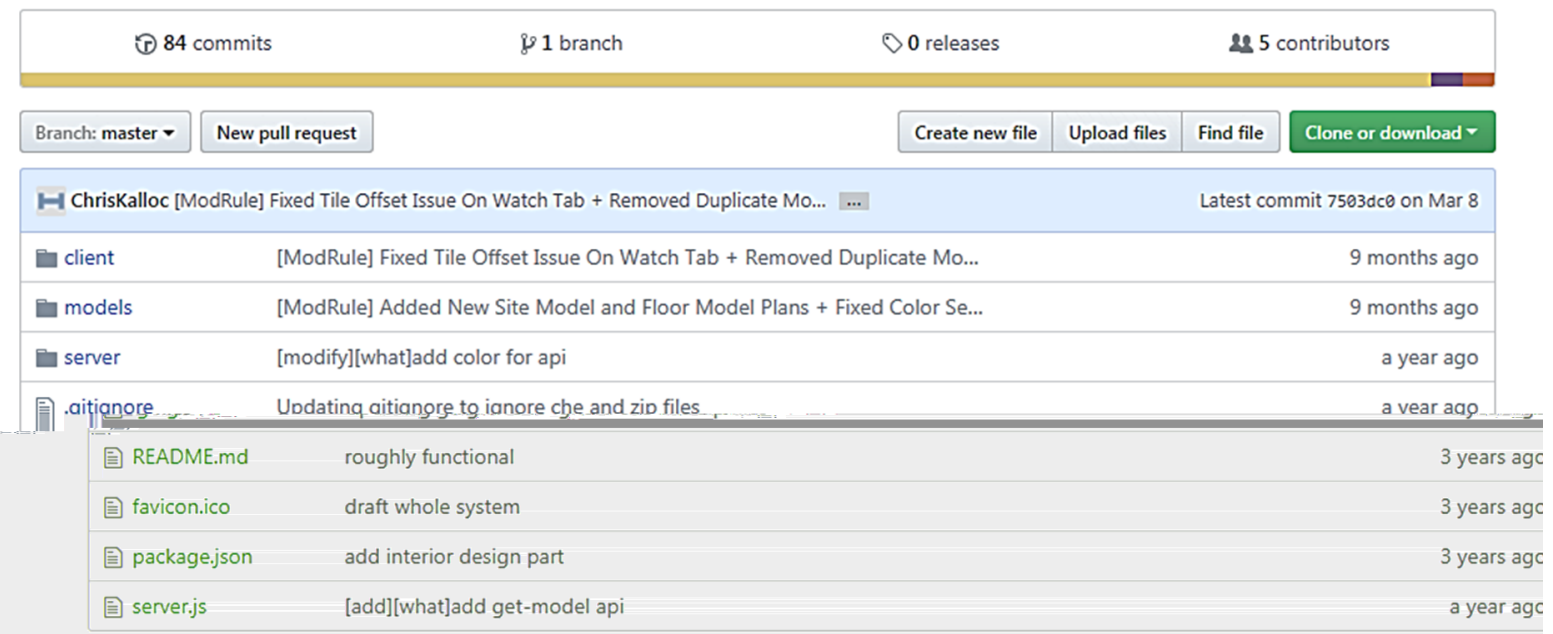

2. Contributors of ModRule in GitHub.

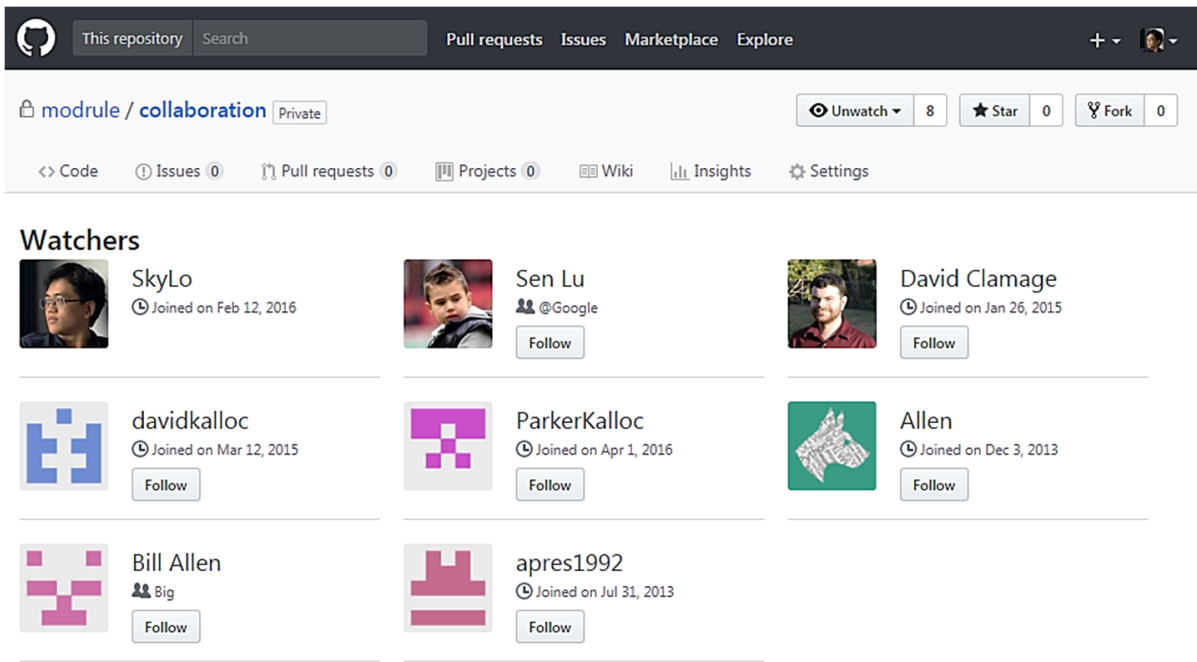




\section{Server using DigitalOcean}

DigitalOcean is a cloud server platform. ModRule uses it to store the data and information generated in the system.

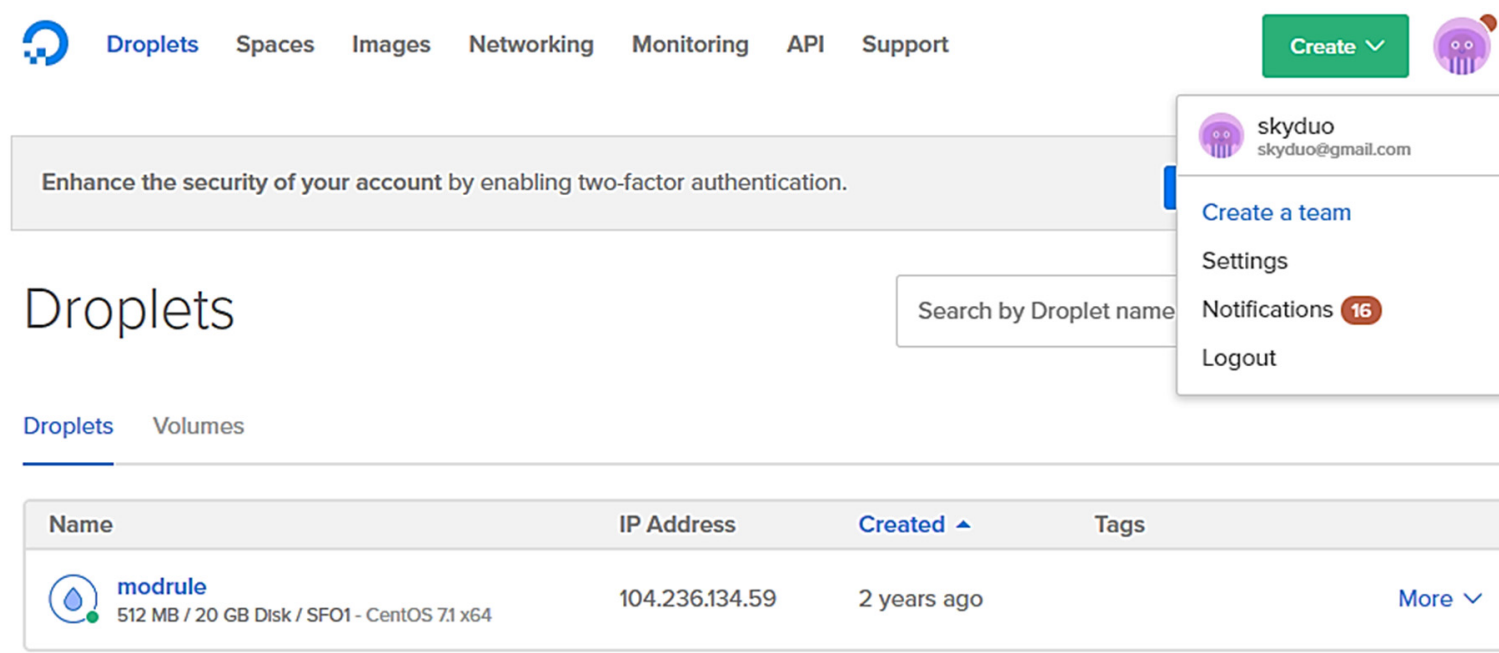

(0) modrule

512 MB Memory / 20 GB Disk / SFO1 - CentOS 7.1 x64

ipv4: 104.236.134.59 ipv6: 2604:a880:1:20:172:1001 Private IP: Enable now Floating IP: Enable now

Graphs
Access
Power
Volumes New:
Resize
Networking
Backups
Snapshots
Kernel
History
Destroy
Tags

Learn how to update this Droplet for new metrics.

Bandwidth public

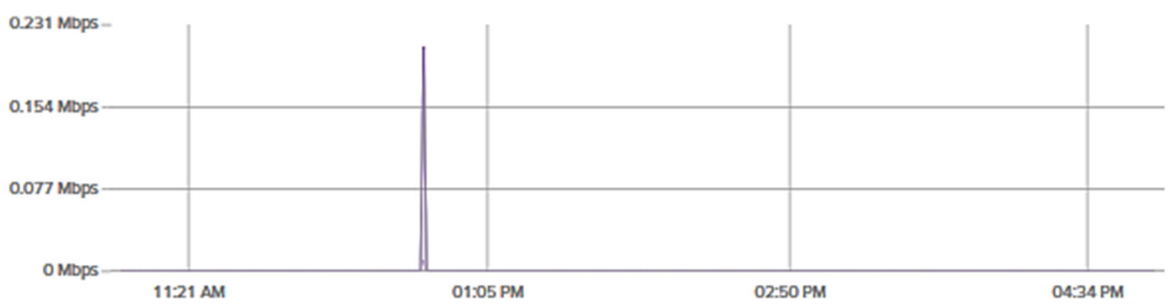

Tags

CPU

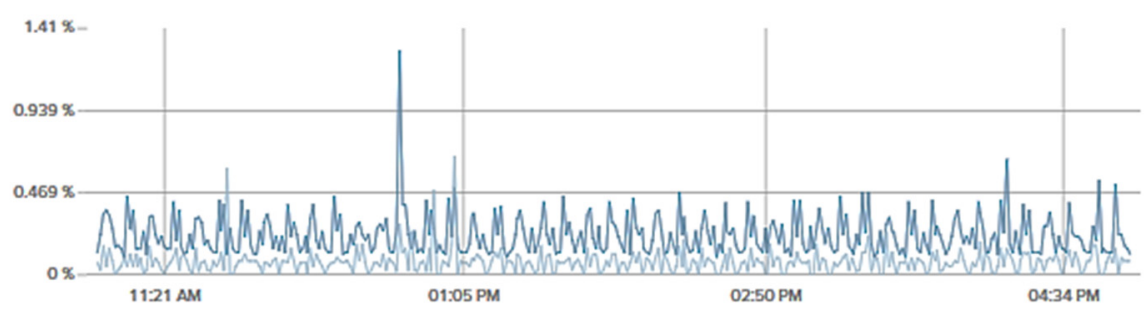

Disk I/O

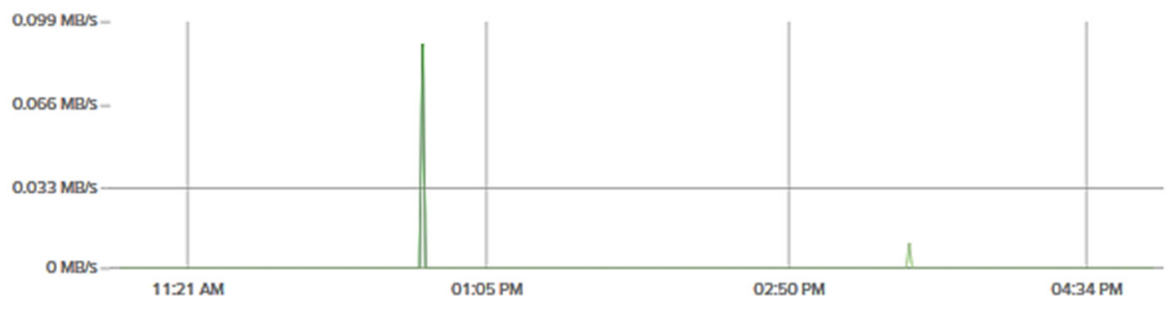




\section{Pre-Development Focus Group Study}

This study was held between August and November 2013. This section includes the images and output of the design process with the focus group.

\section{Introduction to the Study}

1. These are the introduction slide information presented to the participants of the focus group study.

\section{Introduction}

- Use of Computational Architecture Design Techniques

- In association with current research

- New methods of design

- Mass Housing

- Details in Course outline

- Social Media Communication

- Possible Competition Submission: [AC-CA]

http://www.ac-ca.org/en/project08
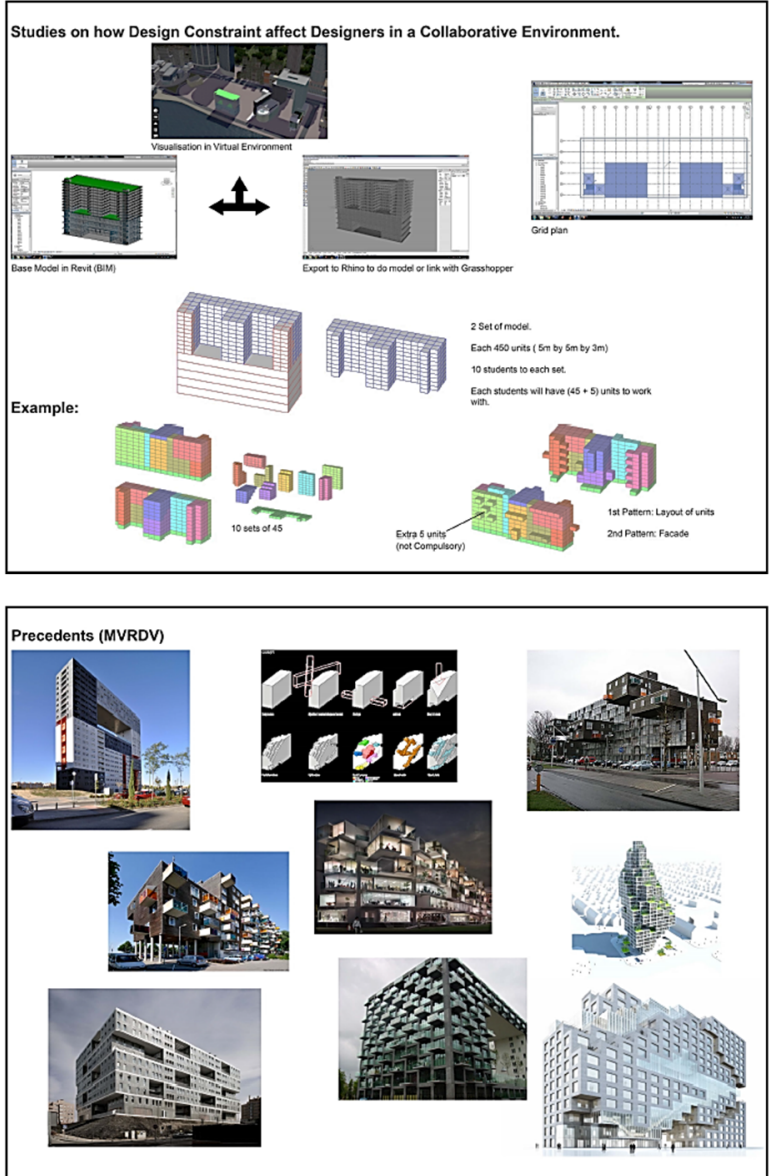

\section{Outline}

Phase 1: Precedence Studies

In the first Phase you analyse patterns of precedents. Hereby you study closely the relationships an

- Phase 2: Massing Studies

In the second Phase develop massing studies of the presented and given model and system. Hereby you
explore the possibilities you have in the given context.

- Phase 3: Digital Modelling

in the third phase you combing your studies of phase 1 and 2, negotiate with your neighbours, and
develop your own system that matches the context.

Phase 4: Facade Studies

Phase 4: Facade Studies
Based on the model of Phase 3 you develop a façade study and combine it with your neighbours to one
common matching composition,

- Phase 5: 3D Printing

Des and panels.
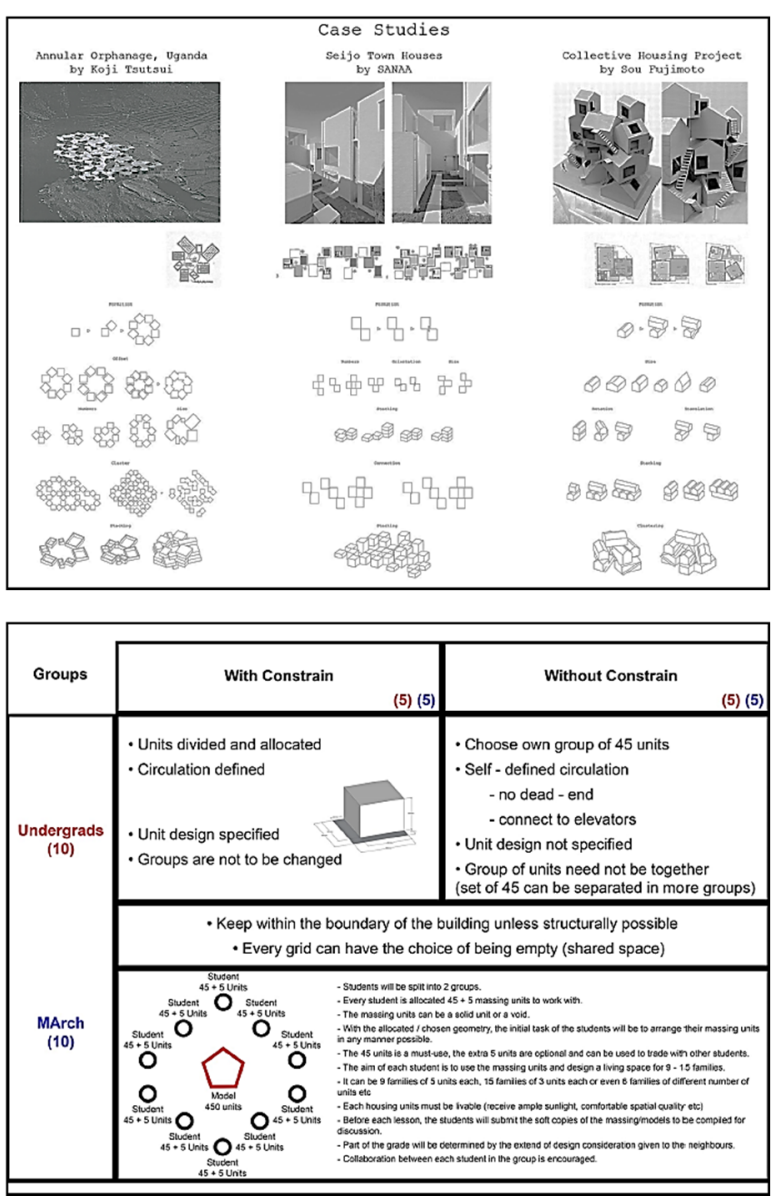

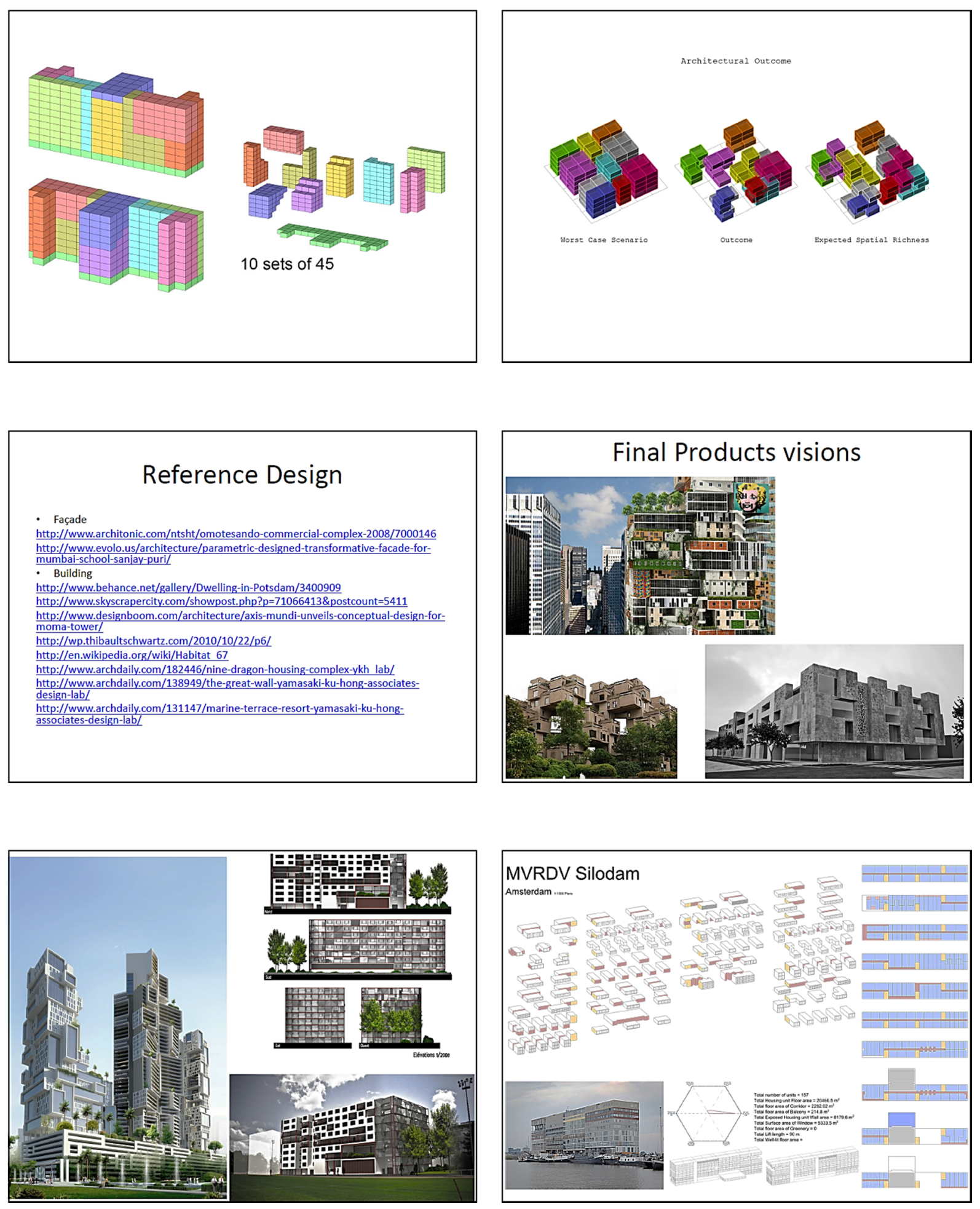


\section{Social media group}

This is a Facebook group created to communicate with the participants. This can be found in https://www.facebook.com/groups/OandP13/.

1. Introduction of the Facebook page.

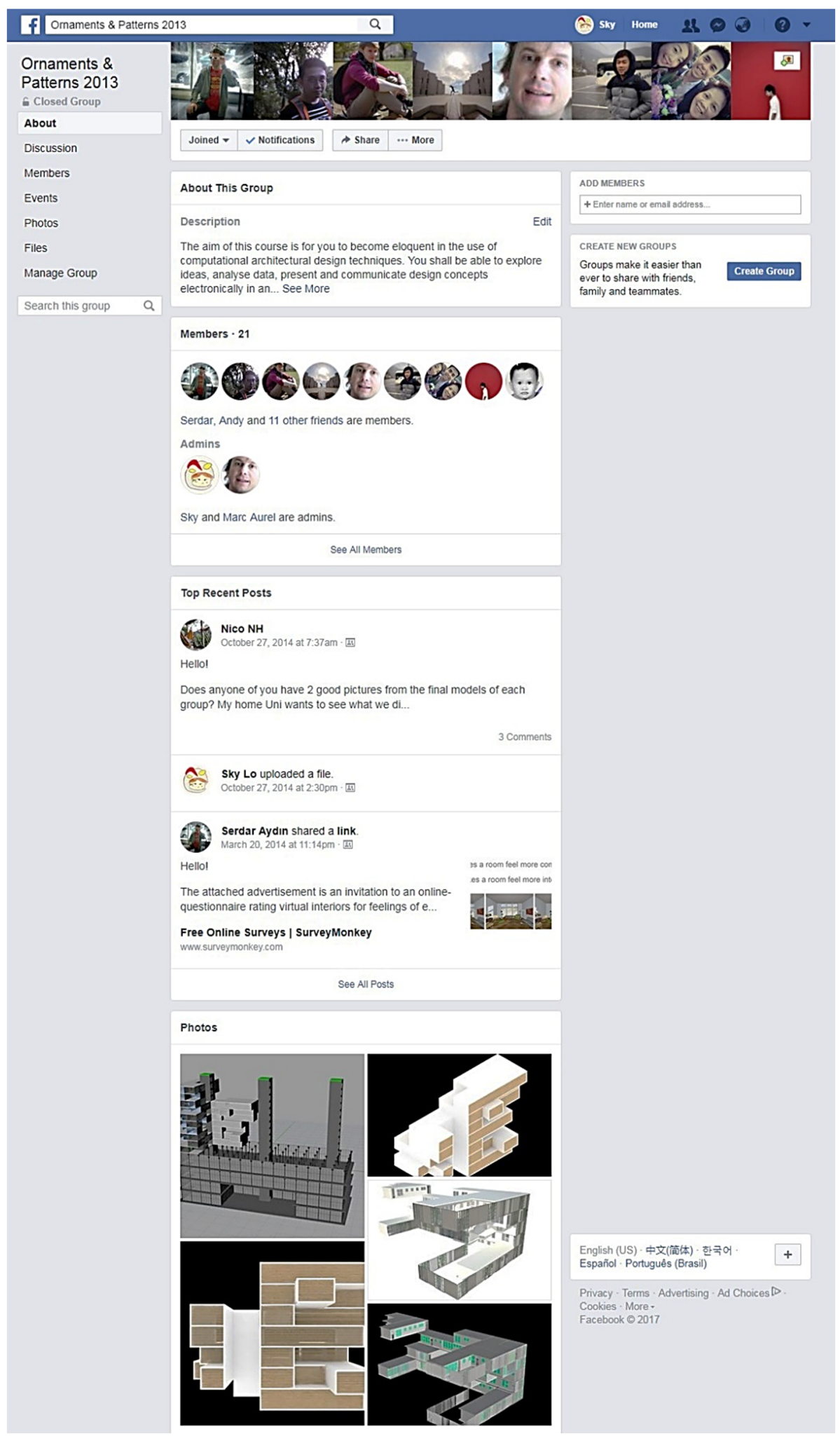


2. Communication within the social media platform indicating the need of a communication medium.

\section{Ornaments \&} Patterns 2013

a Closed Group

\begin{tabular}{l} 
About \\
\hline Discussion \\
Members \\
Events \\
Photos \\
Files \\
Manage Group \\
\hline Search this group \\
\hline
\end{tabular}
(2)
Write a comment.
(-)

Taylor Williams

December 5, 2013

\section{Group 1, can we meet Friday?}

॥b Like $\square$ Comment

You, Nico NH and Leo Yu

Seen by everyone

\section{(8)}

Erin Lau I am okay

See Translation

December 5, 2013 at 3:20am - Like

Phila Fung Me too

December 5, 2013 at 5:08am - Like

A Hansen Chan Okay . At what time? 12 ?

December 5, 2013 at 2:53pm - Like

Nico NH 12 is good! where?

December 5, 2013 at 8:07pm - Like

Leo Yu AITB Com lab?

December 5, 2013 at 9:47pm - Like

Nico NH Fine! see you there!

December 5, 2013 at 9:49pm - Like

Hansen Chan Fine!

December 5, 2013 at 9:56pm - Like

(..) Leo Yu good

December 5, 2013 at 9:56pm - Like

Phila Fung sorry guys can only do after 2 .

can we meet at 2 ?

December 5, 2013 at 10:44pm - Like

Leo Yu Don't worry, you can join us around $2 \mathrm{pm}$.

December 6, 2013 at 12:54am · Like

Taylor Williams fine, be there at 12

December 6, 2013 at 6:24am - Like

- Justin Chan ok

December 6, 2013 at 2:39pm - Like

Write a comment..

(-) 0 (다 $\theta$ 


\section{Progress of Study}

The progress of the focus group study is recorded through images taken at different stage of the process.

1. Getting started with physical cubes.

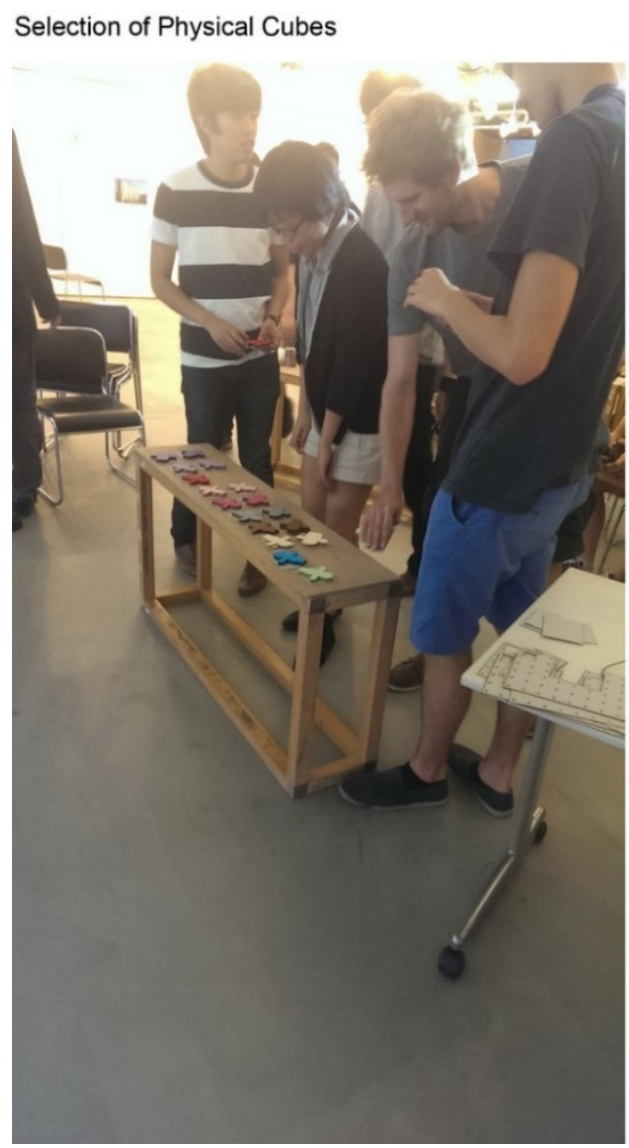

Determination of the group memebers

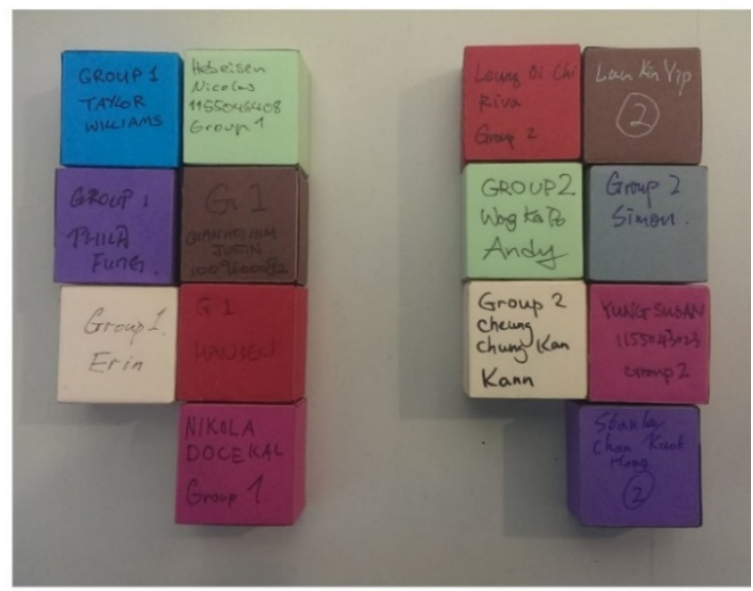

Setup of the venue for the design collaboration process
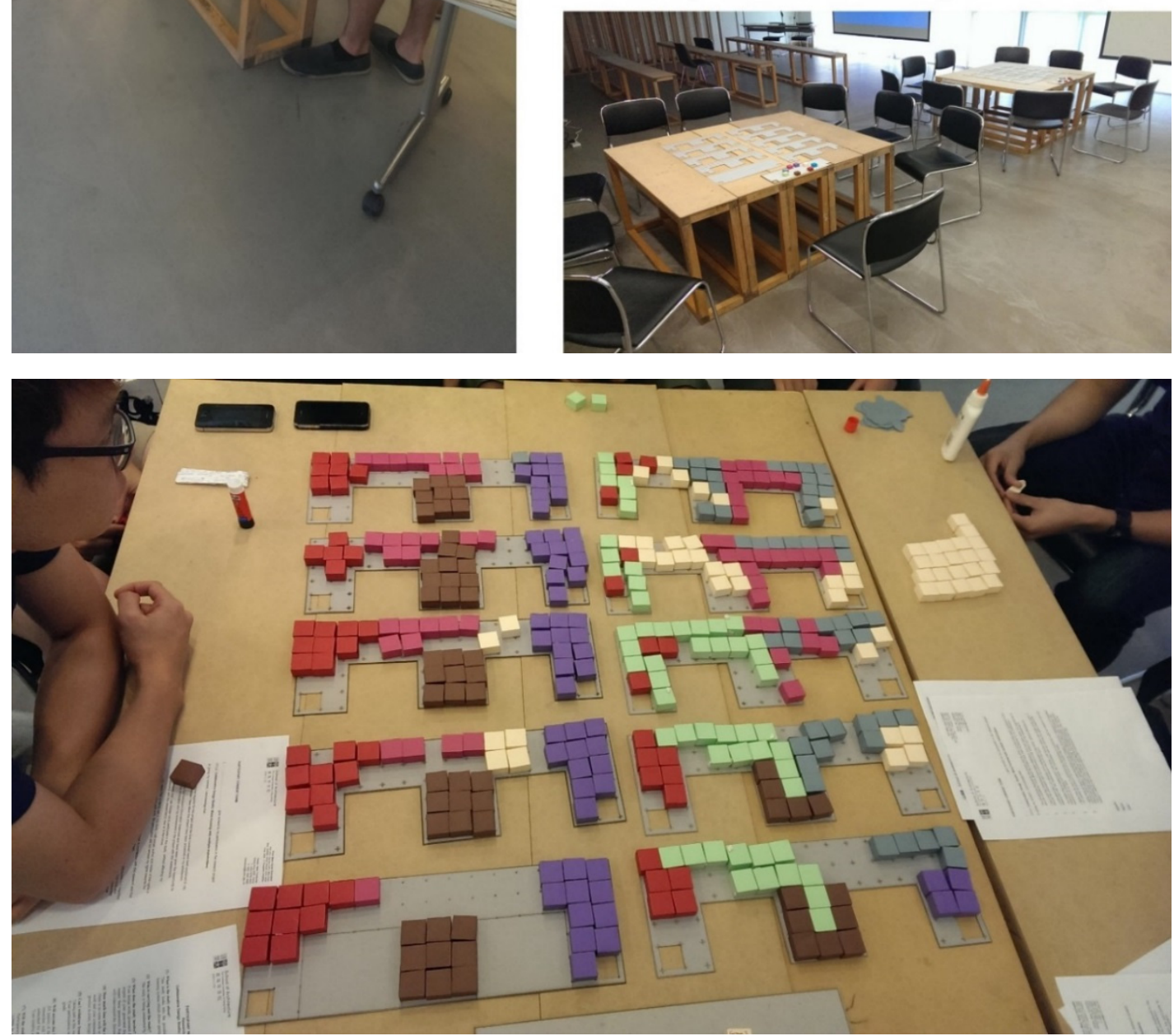

Design discussion using physical cubes. 
2. Getting into discussion of the plans to work out the details.

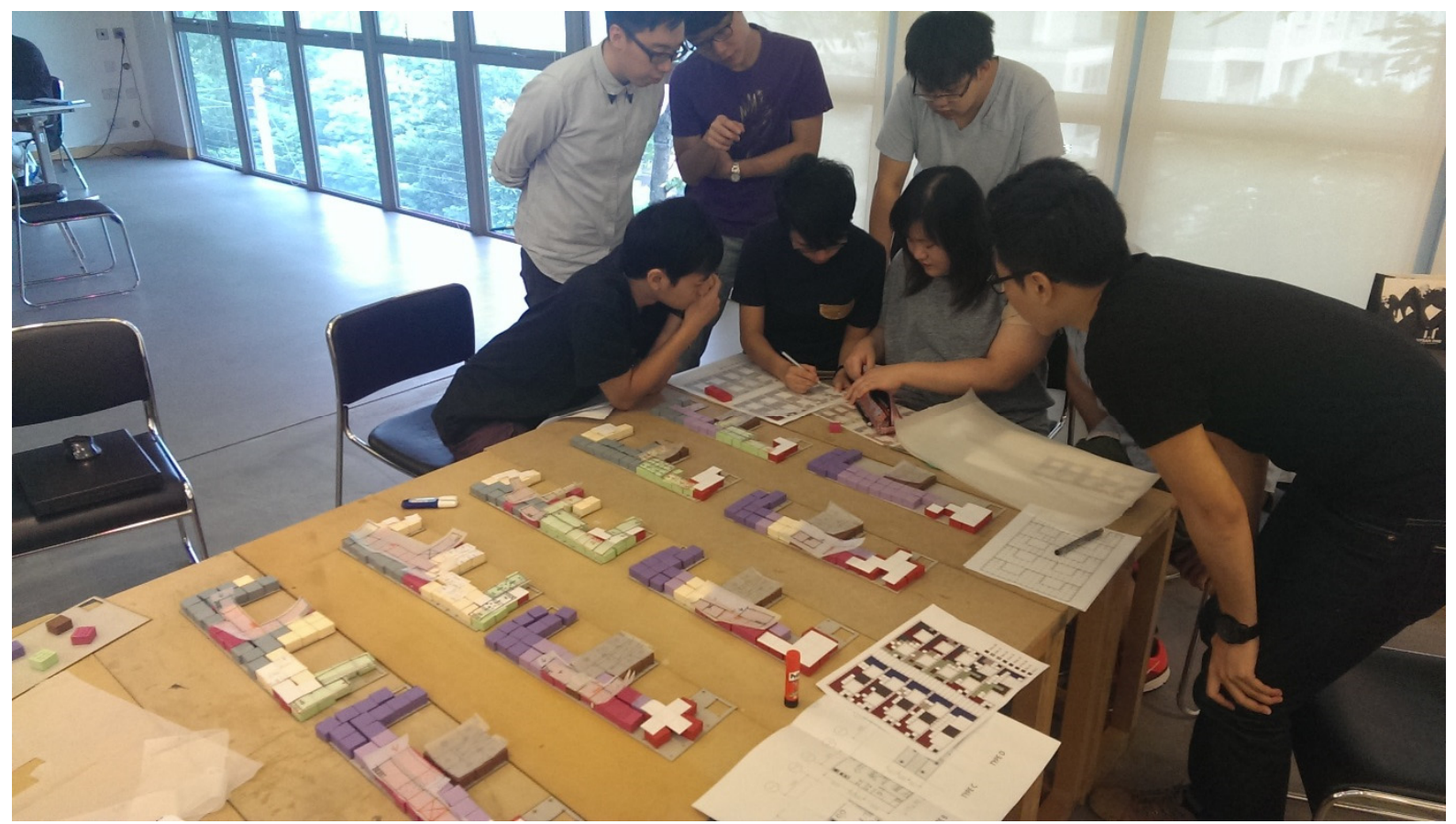

3. Results from one of the discussion session showing the plans, connected walkways and shared spaces.
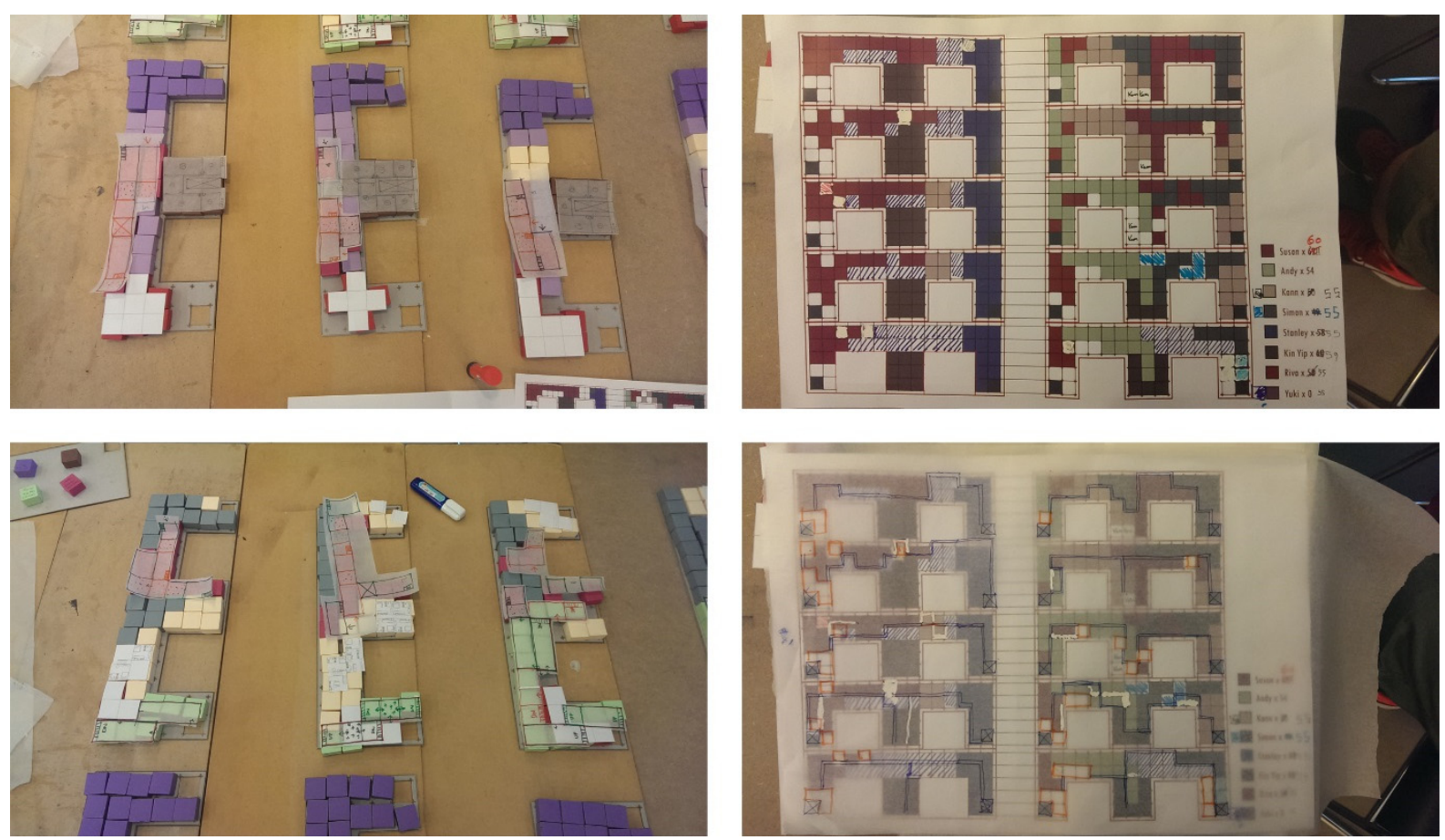
4. Stacking the cubes to examine the outlook.

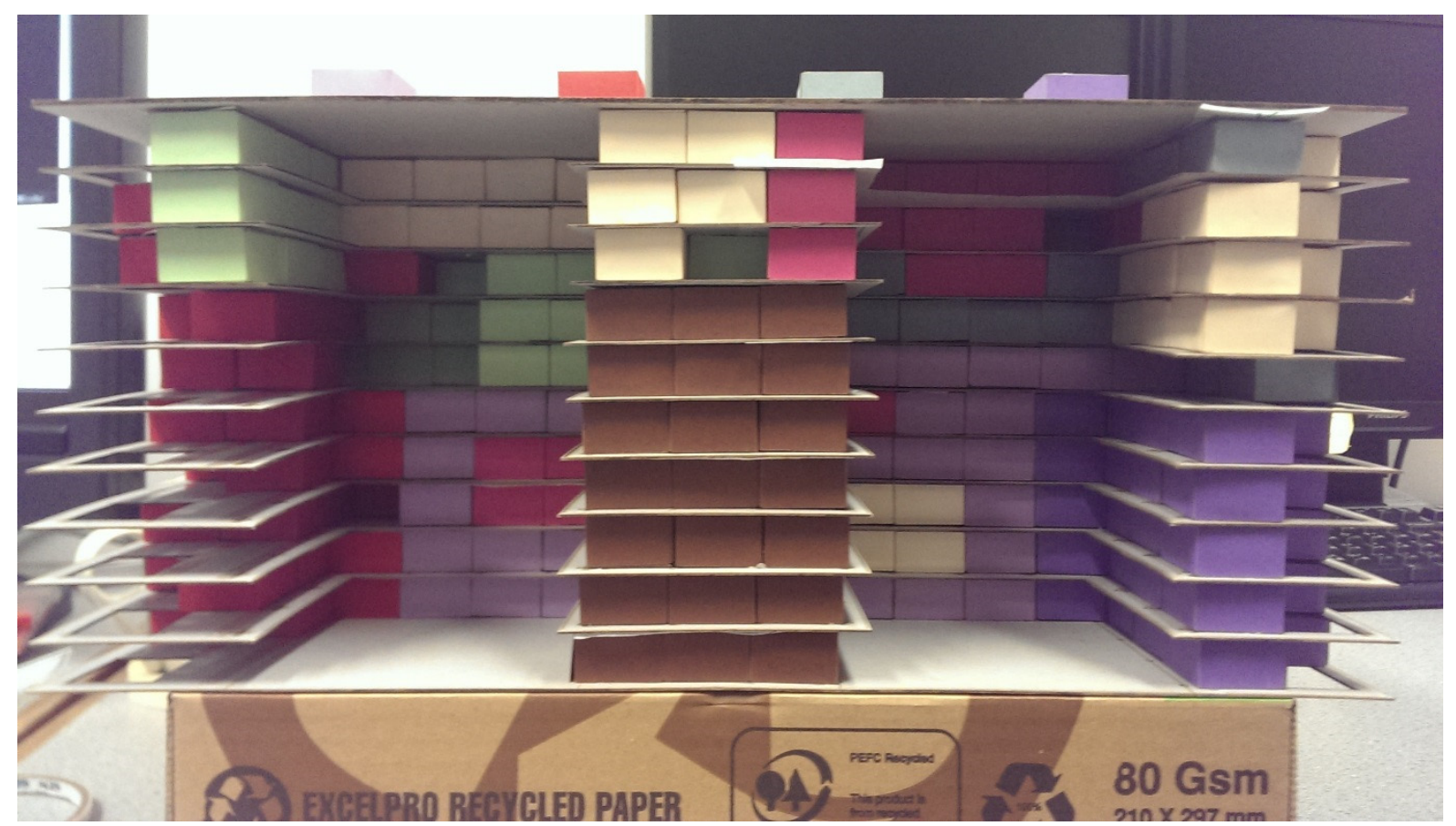

5. Working out the models and the exterior outlook of the overall design.

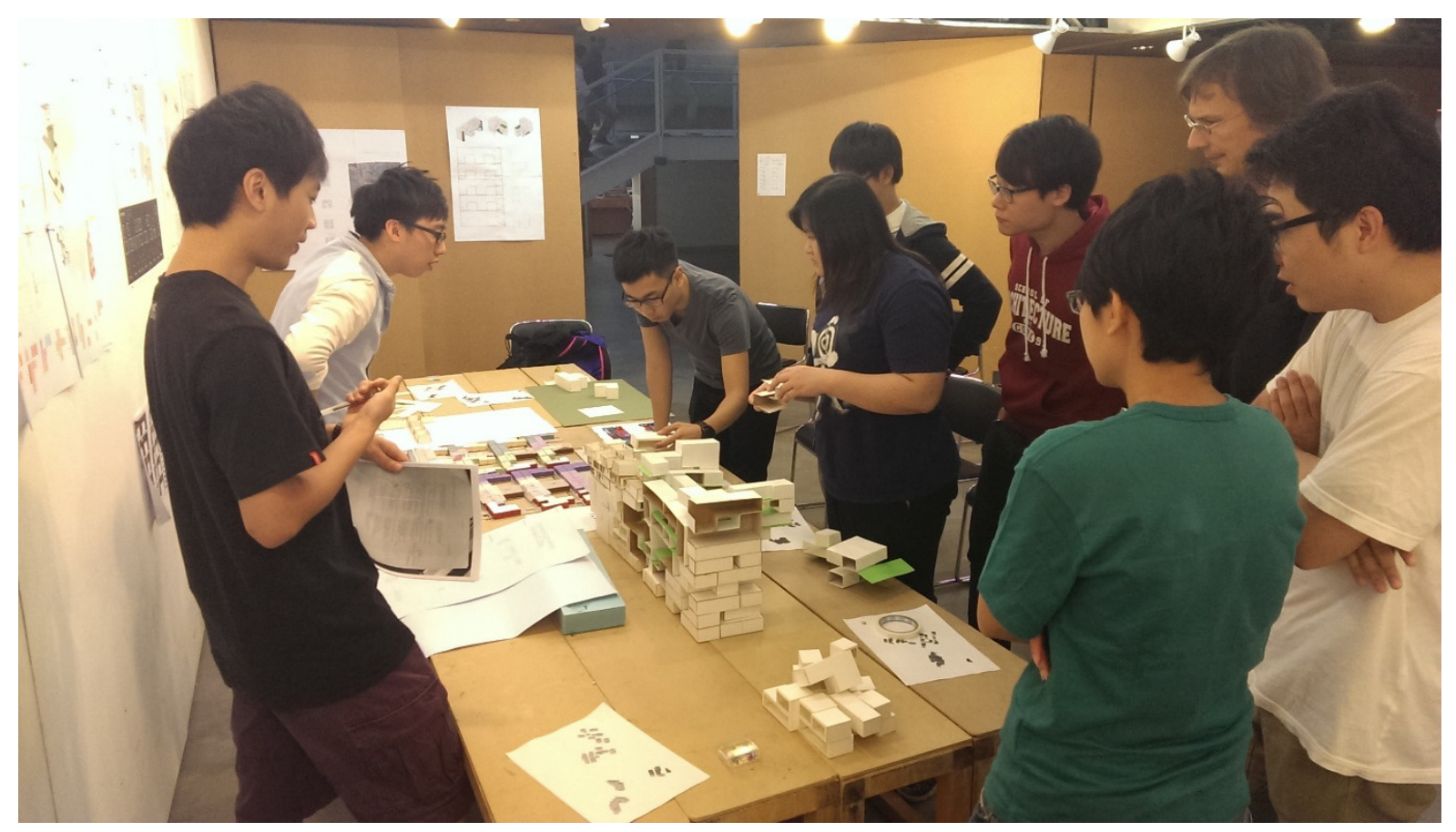


6. The collective results of the 8 participants in one group, with one of the designs enlarged.

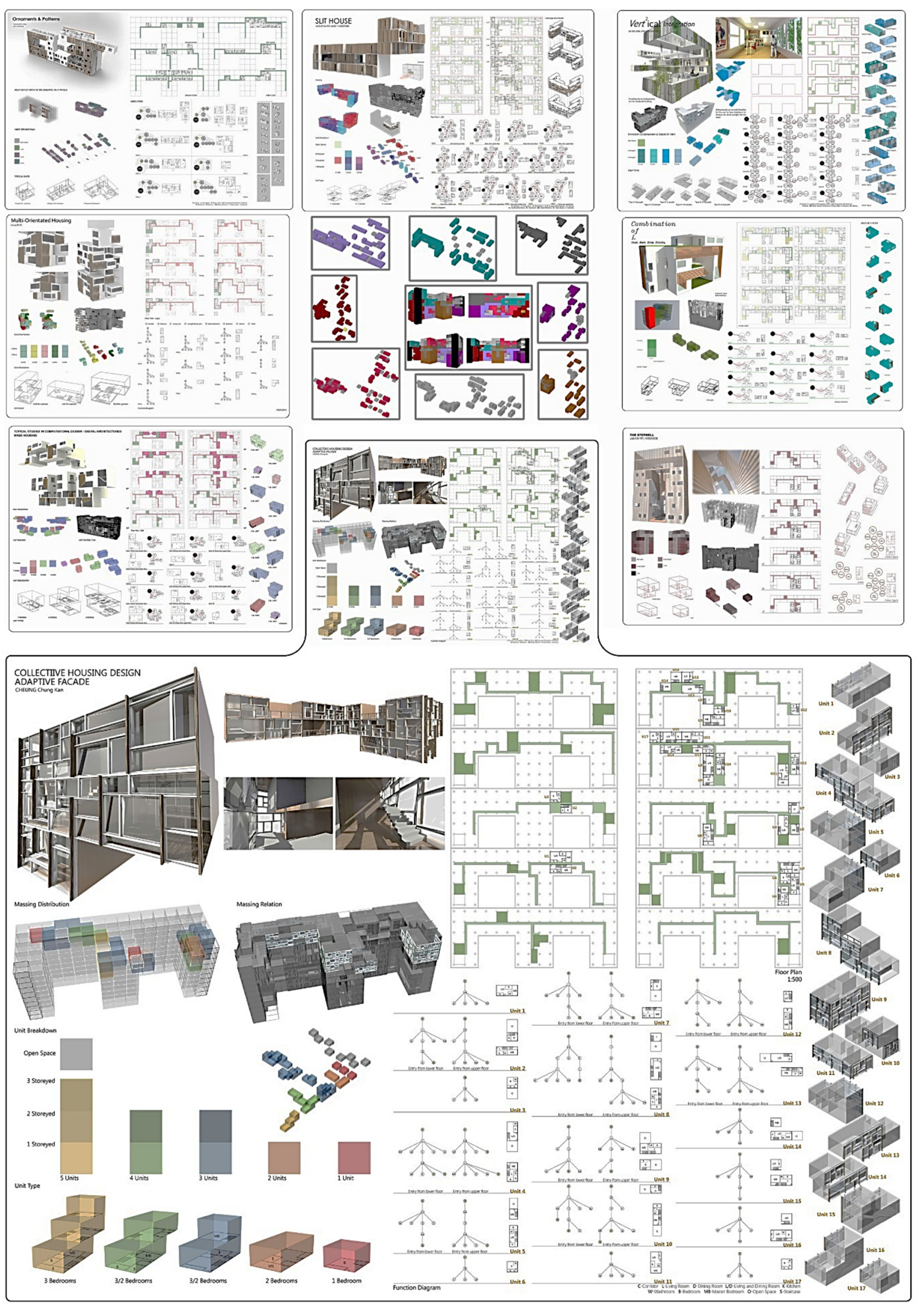


7. The final rendered outcome based on the collective design of one group.

\section{Adaptive Verticality oy rean Humanante}

Where process is more important than the design
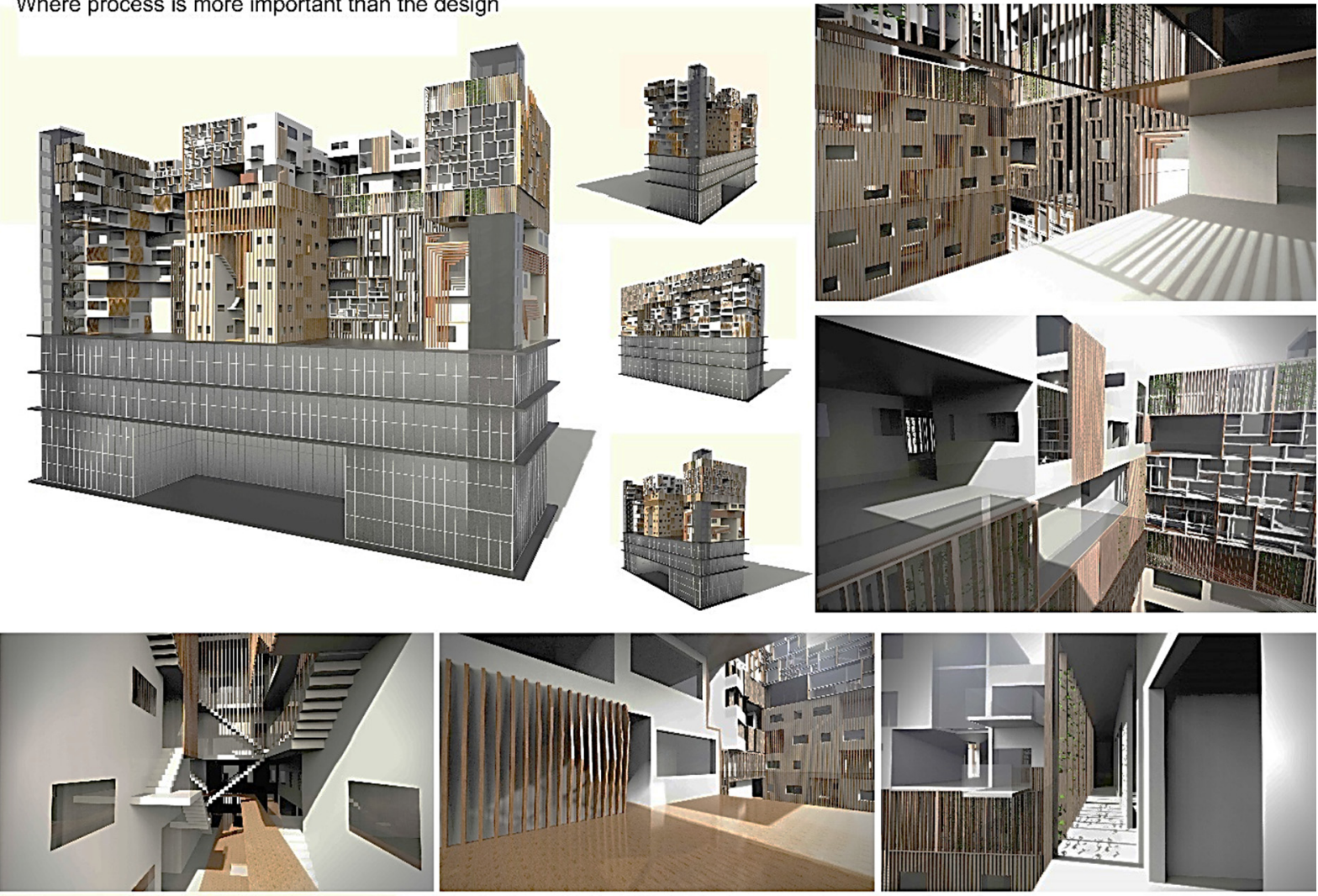

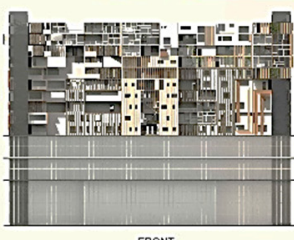

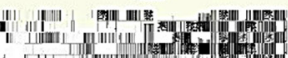
in Viniti
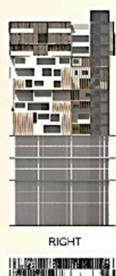

$x$

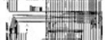

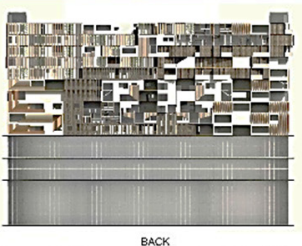

BACK

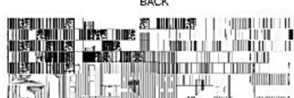

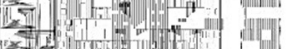
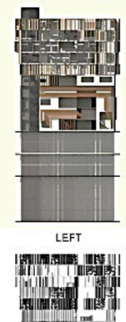

$=$
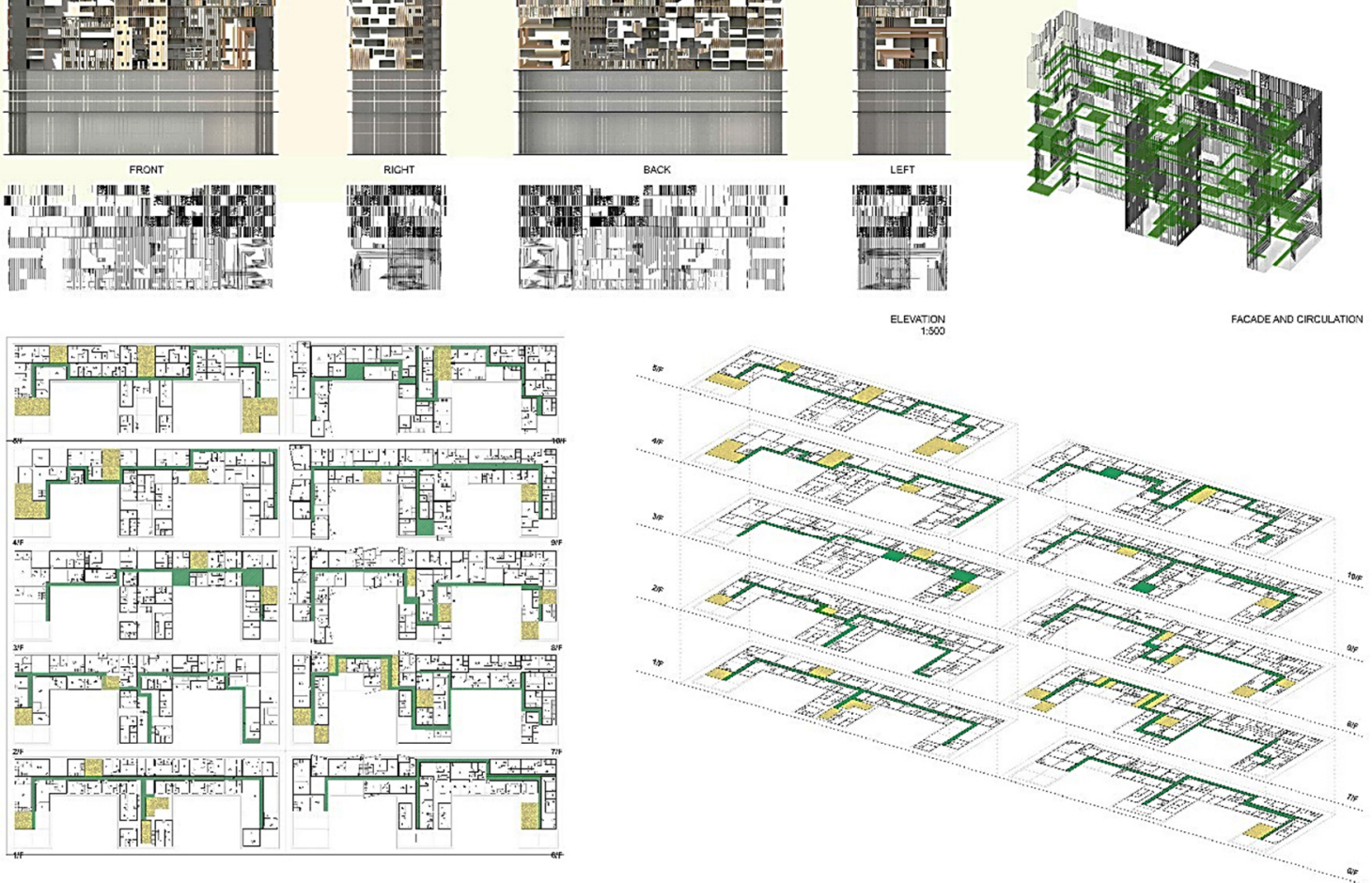

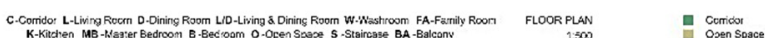


8. Comparison of the two designs generated by the two groups of participants.

\section{Digital generation of outcome}
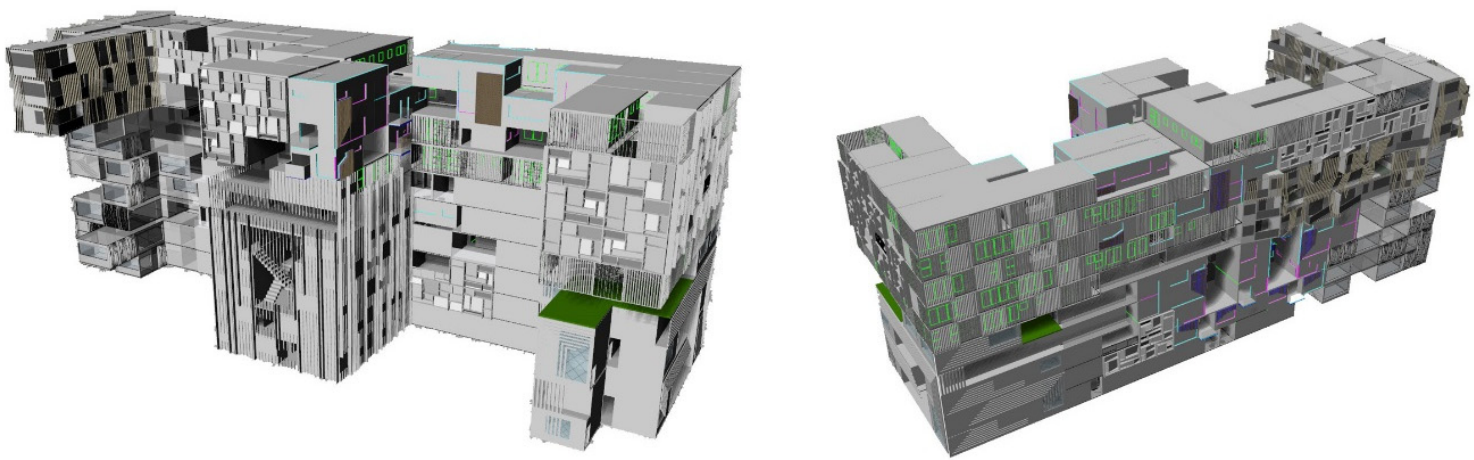

Group 1 Outcome

Group 2 Outcome
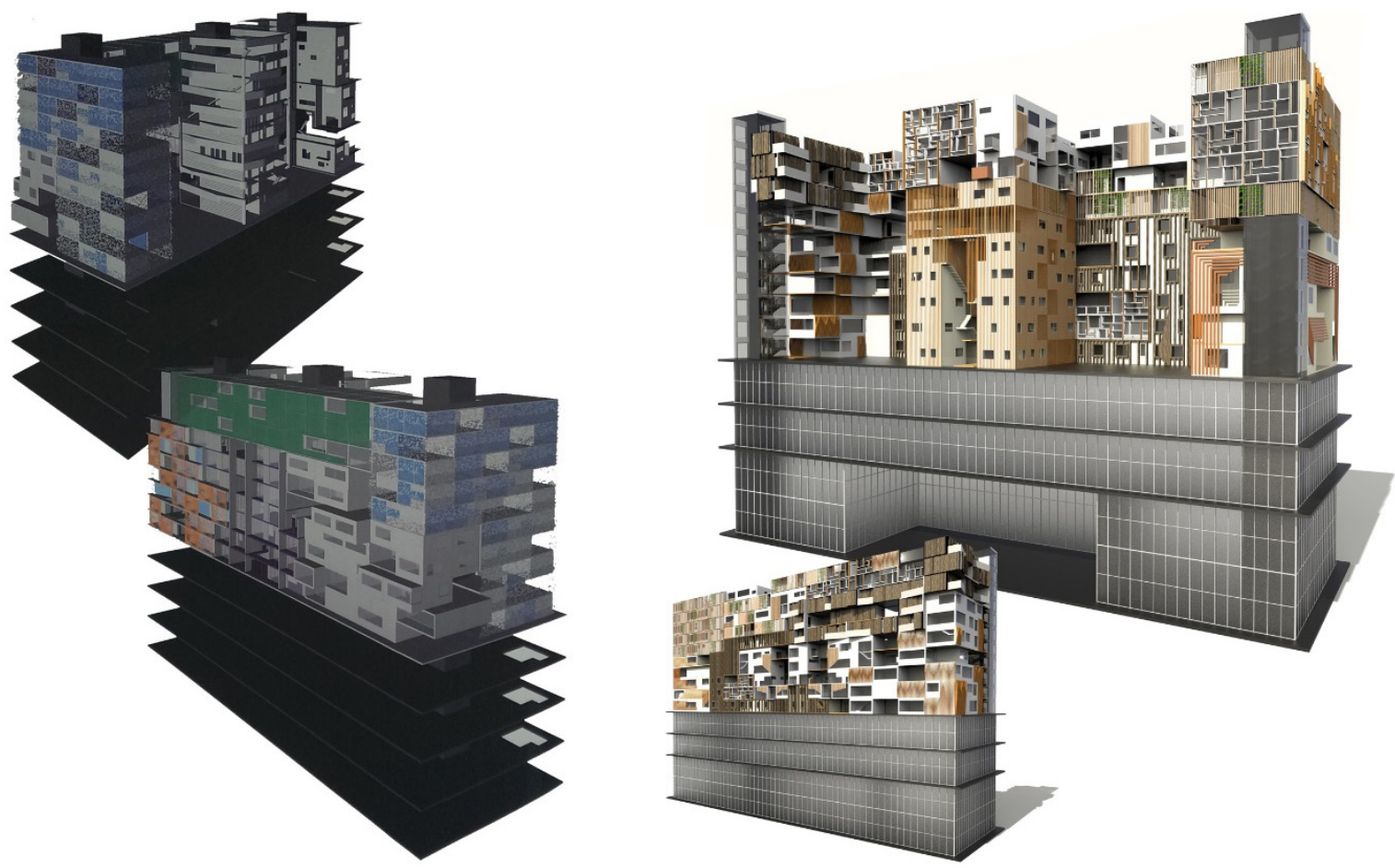
9. Presenting the ideas to a general group of people.

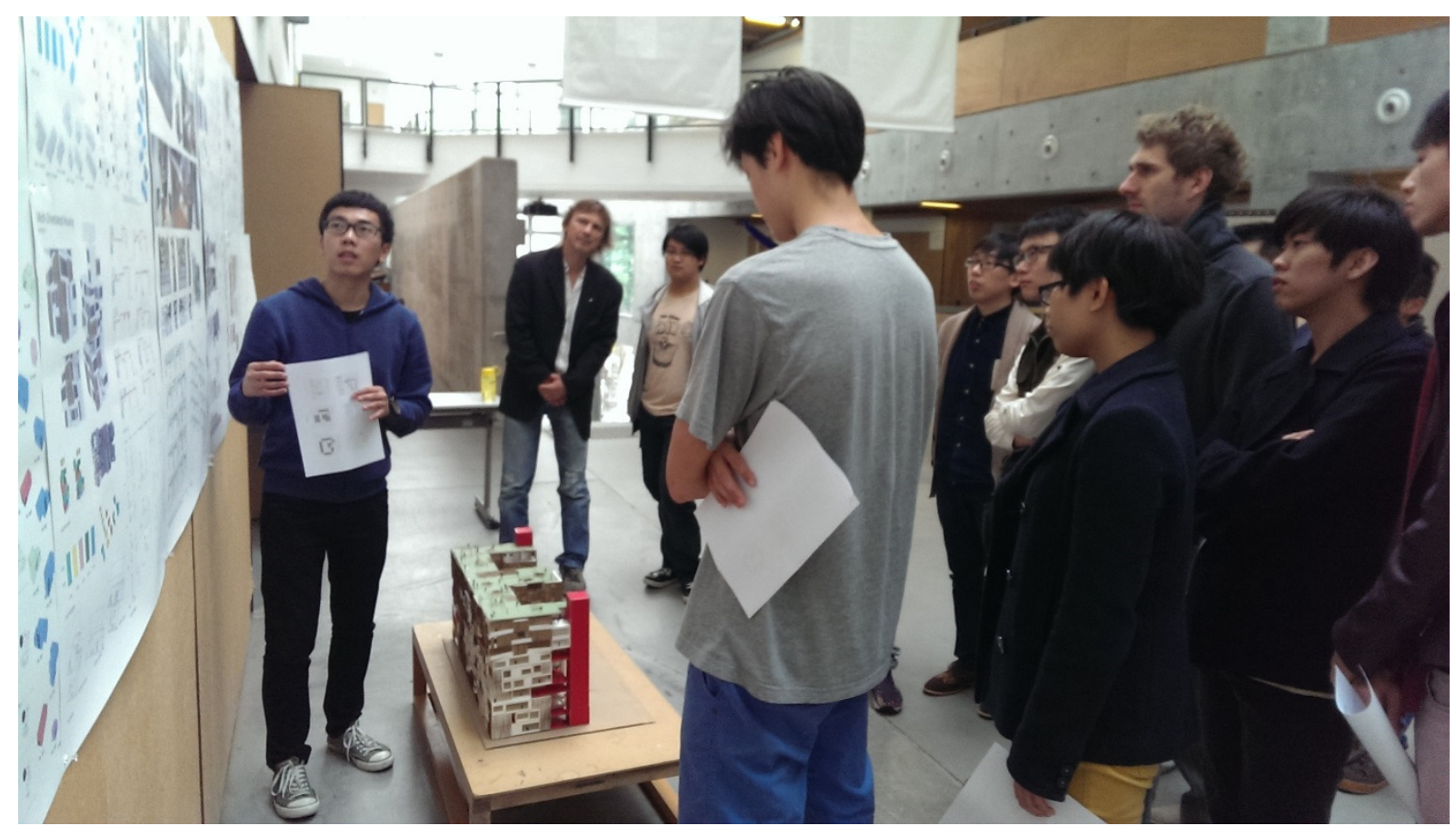

10. Progression model of one of the groups.

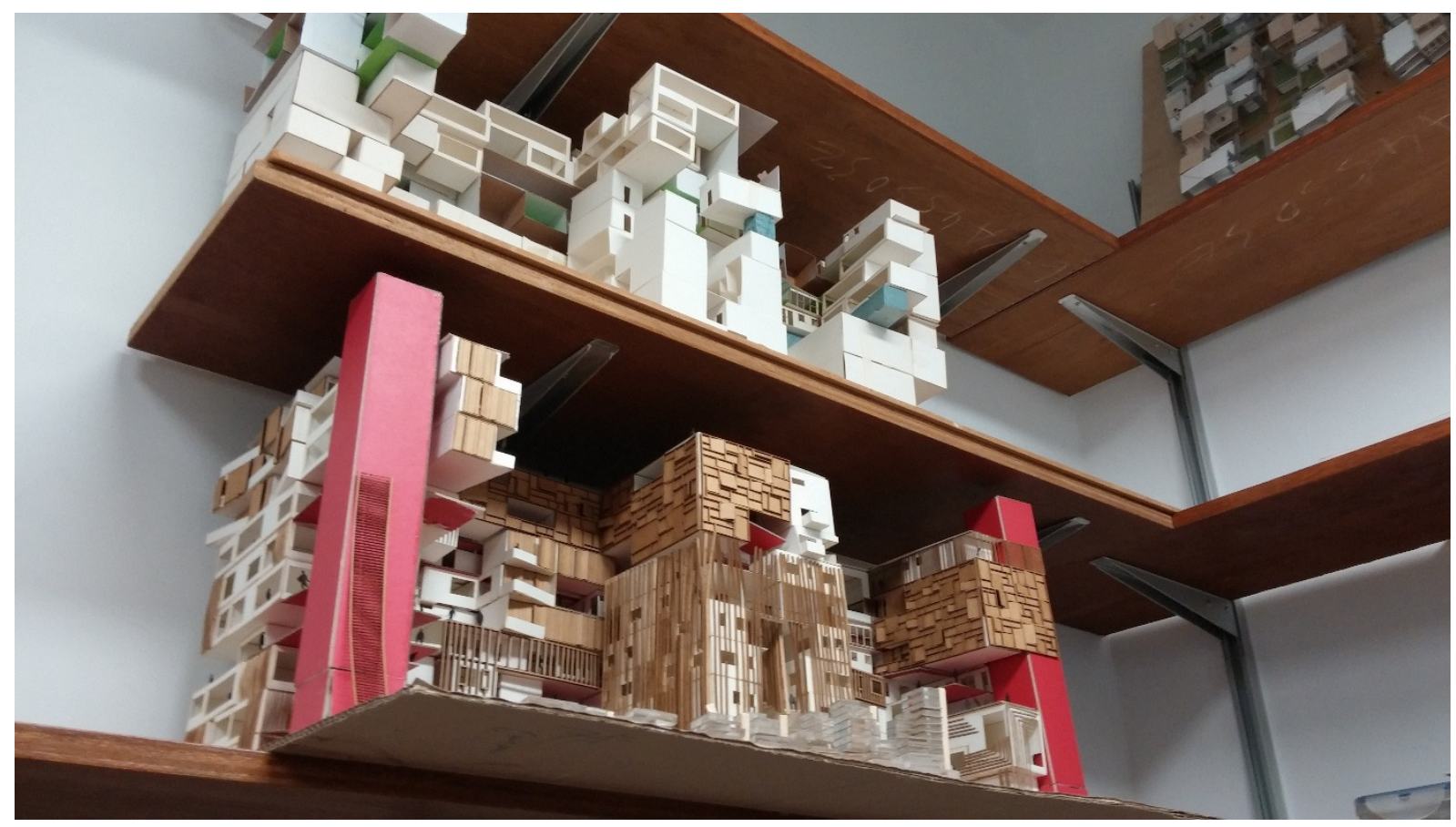


11. The physical outcome of the two groups exhibited for viewing.
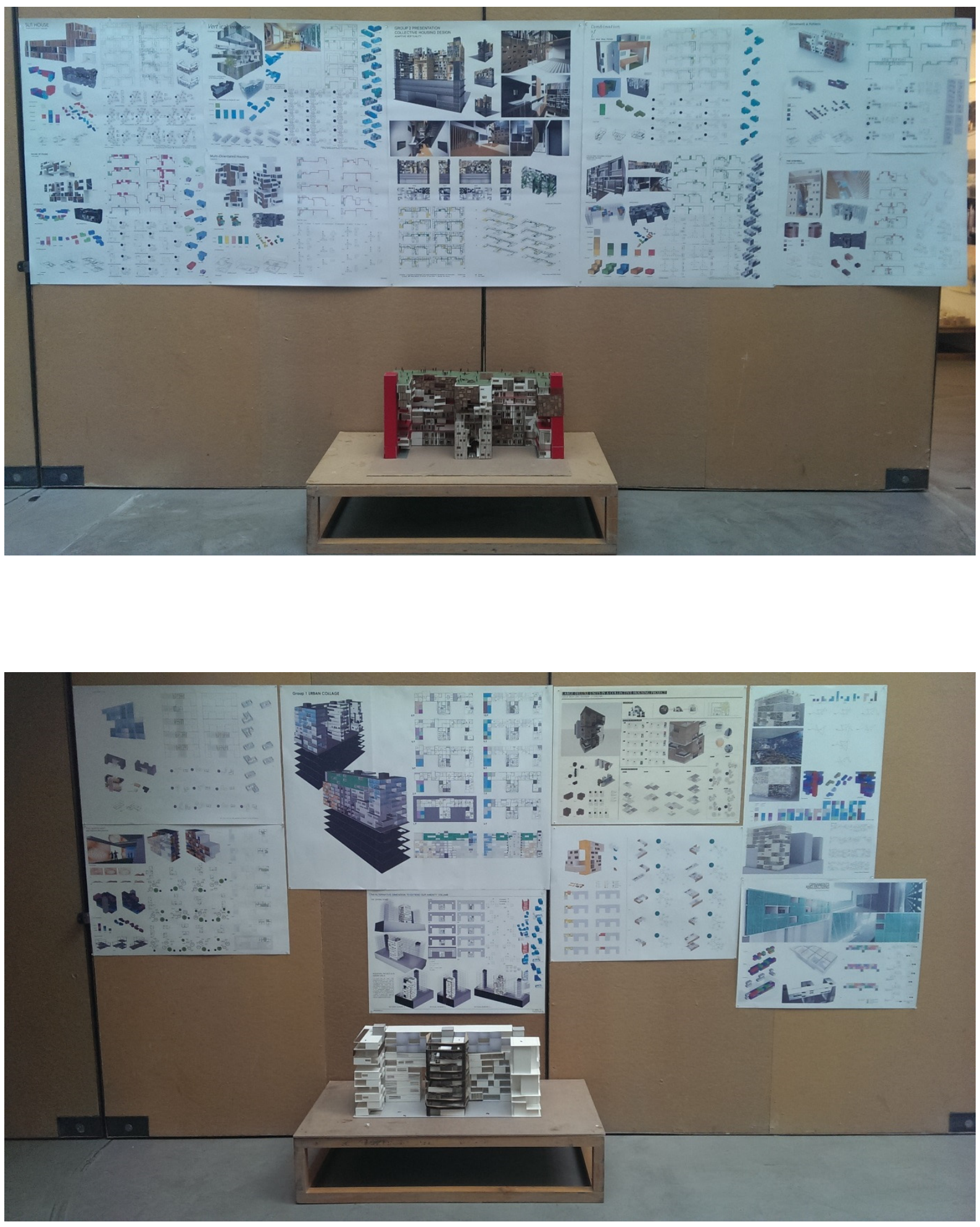


\section{Data Collection}

1. Questionnaires given to participants after the design study.

\section{Survey for Patterns and Ornaments 2013 \\ Collaborative Design Studio: Mass Housing with Multiple Stakeholders \\ Residential Context}

1. How do you find this design method?

\begin{tabular}{|l|ll|ll|}
\hline$\square \quad$ Boring & $\square$ & Interesting & $\square$ & Challenging \\
\hline
\end{tabular}

2. If there is a web integrated communication tools for discussion with $3 \mathrm{~d}$ visualization, how much do you think will help this design process?

\begin{tabular}{|c|c|c|c|c|}
\hline$\square$ None & $\square$ Not much & $\square$ OK & $\square$ Quite & $\square$ Very much \\
\hline
\end{tabular}

3. How do you think can improve this design process?

Suggestions

4. How much would you use this idea as a thesis?

\begin{tabular}{|c|c|c|c|c|}
\hline$\square$ None & $\square$ Not much & $\square$ OK & $\square$ Quite & $\square$ Very much \\
\hline
\end{tabular}

5. As a potential occupant, how much do you want to be involved in the design process for your own place?

\begin{tabular}{|c|c|c|c|c|}
\hline$\square$ None & $\begin{array}{c}\square \text { Not much } \\
(30 \%)\end{array}$ & $\begin{array}{c}\square \text { OK } \\
(50 \%)\end{array}$ & $\begin{array}{c}\square \text { Quite } \\
(70 \%)\end{array}$ & $\begin{array}{c}\square \text { Very much } \\
(90 \%)\end{array}$ \\
\hline
\end{tabular}

6. As a user, how simplified the tools you want to interact with the design

\begin{tabular}{|c|c|c|c|c|}
\hline $\begin{array}{c}7 \text { Detailed } \\
\text { (Grid checking) }\end{array}$ & $\begin{array}{l}\text { (1) Challenging } \\
\text { (Components) }\end{array}$ & $\begin{array}{c}\text { Average } \\
\text { (Drag \& Drop) }\end{array}$ & $\begin{array}{l}\text { Quite Simple } \\
\text { (Sliders) }\end{array}$ & $\begin{array}{c}\text { Very Simple } \\
\text { (Templates) }\end{array}$ \\
\hline
\end{tabular}

7. As a designer, how much are you willing to give user the flexibility of design?

\begin{tabular}{|c|c|c|c|c|}
\hline$\square$ None & $\begin{array}{c}\square \text { Not much } \\
(30 \%)\end{array}$ & $\begin{array}{c}\square \text { OK } \\
(50 \%)\end{array}$ & $\begin{array}{c}\square \text { Quite } \\
(70 \%)\end{array}$ & $\begin{array}{c}\square \text { Very much } \\
(90 \%)\end{array}$ \\
\hline
\end{tabular}

8. In a high rise context, to what level of consistency is still considered having an overall appearance?

\begin{tabular}{|l|c|c|c|c|}
\hline$\square$ None & $\square$ Not much & $\square$ OK & $\square$ Quite & $\square$ Very much \\
\hline
\end{tabular}

9. In this studio, what are the greatest difficulties you faced? (arrange from smallest to biggest)

․ Not collaborative team members

․ Tight schedule

$\square$ Design constrains

․ Group size

․ Information gathering

O Others

10. Is the information \& instruction conveyed clearly through Facebook (FB)?

\begin{tabular}{|l|c|c|c|c|}
\hline$\square$ Not Clear & $\square$ Clear & $\square$ Very Clear & $\square$ I don't know & $\square$ Don't use FB \\
\hline
\end{tabular}

11. Is the information \& instruction conveyed clearly verbally?

\begin{tabular}{|c|c|c|c|}
\hline$\square$ Not Clear & $\square$ Clear & $\square$ Very Clear & $\square$ I don't know \\
\hline
\end{tabular}

12. How do you think can improve this studio?

\begin{tabular}{|l|}
\hline Suggestions \\
\end{tabular}


2. Results of the questionnaire done by the sixteen participants in the study.

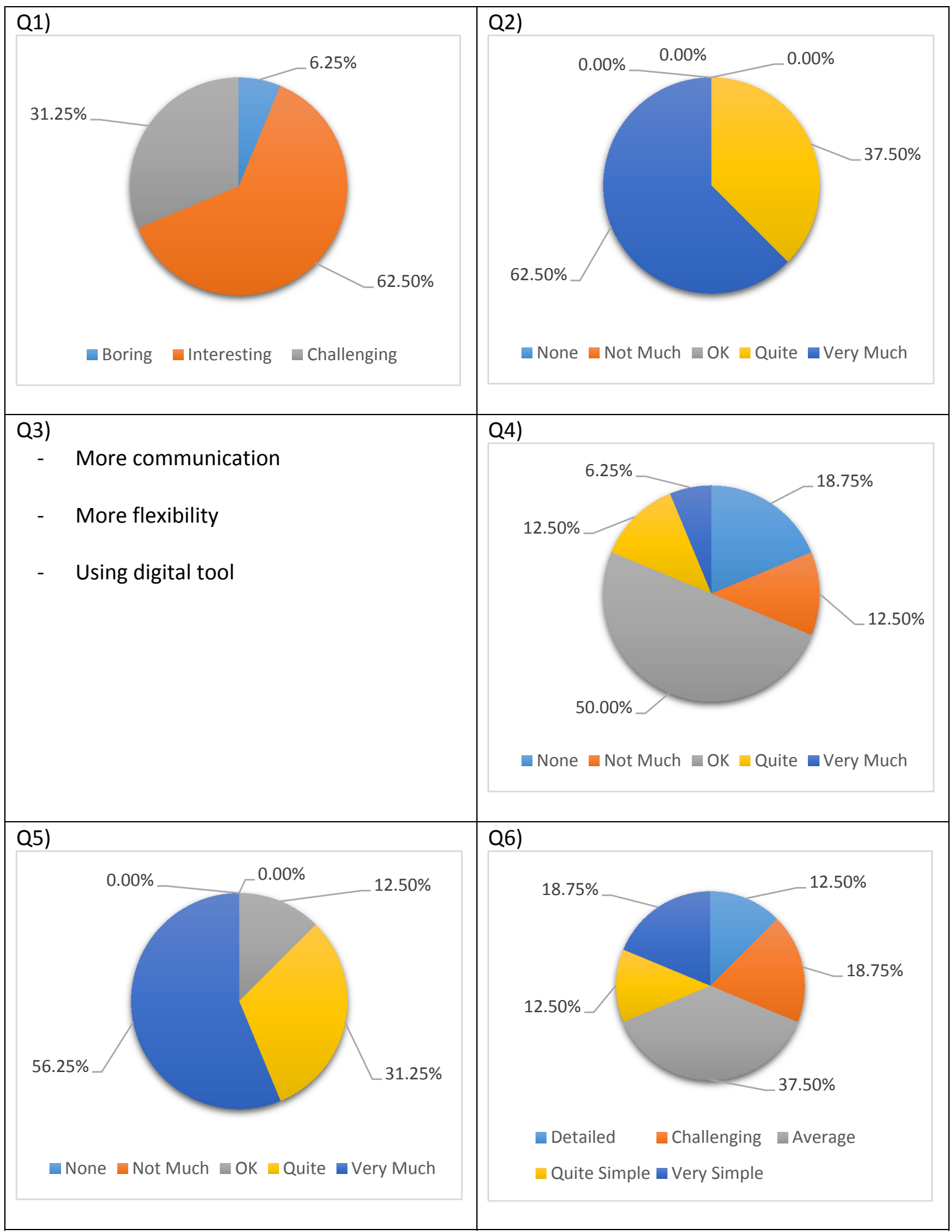




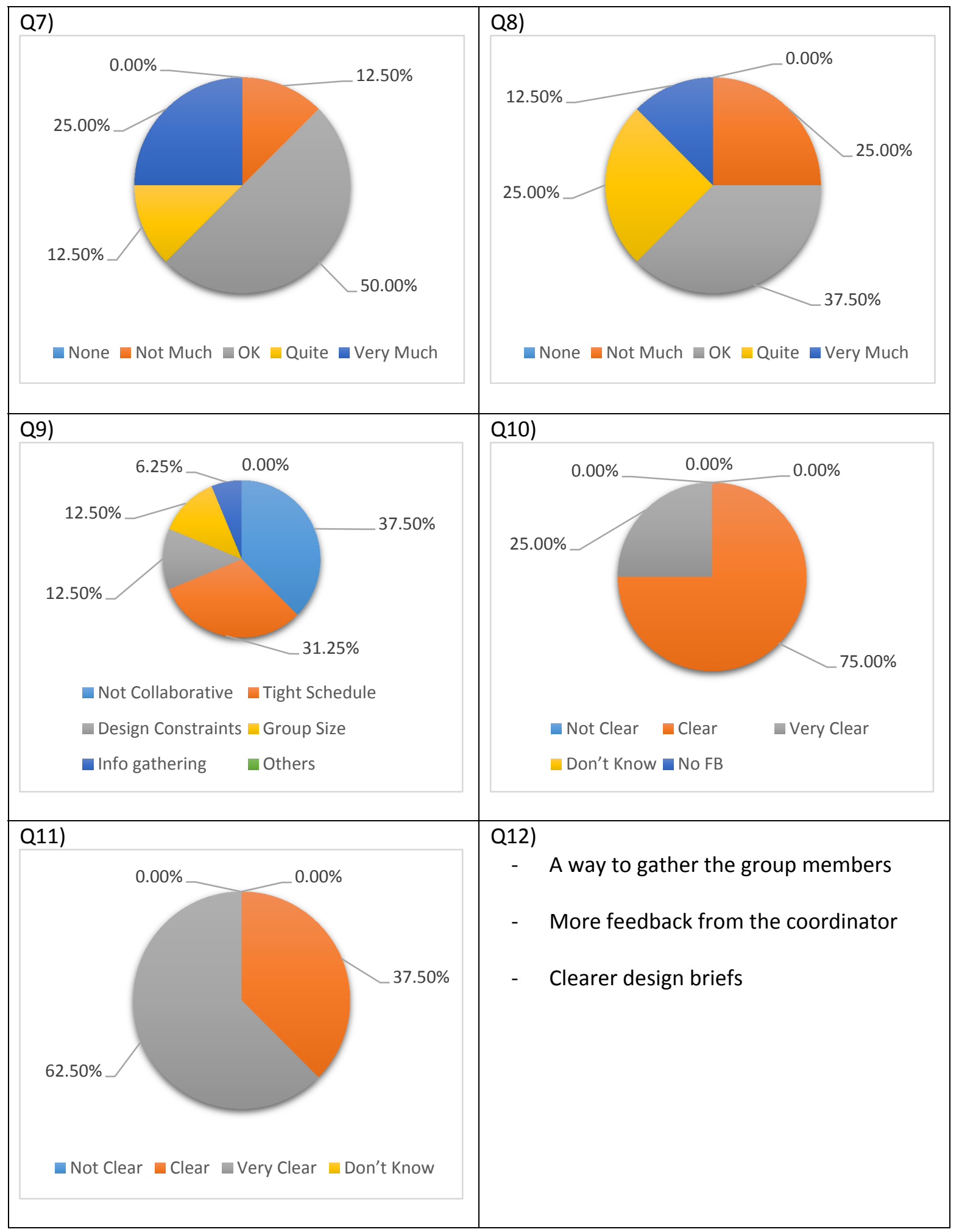




\section{Focus Group Study with ModRule}

This study was held between August and November 2014. This section includes the images and output of the design process with the focus group.

\section{Introduction to the Study}

1. These are the introduction slide information presented to the participants of the focus group study with ModRule.
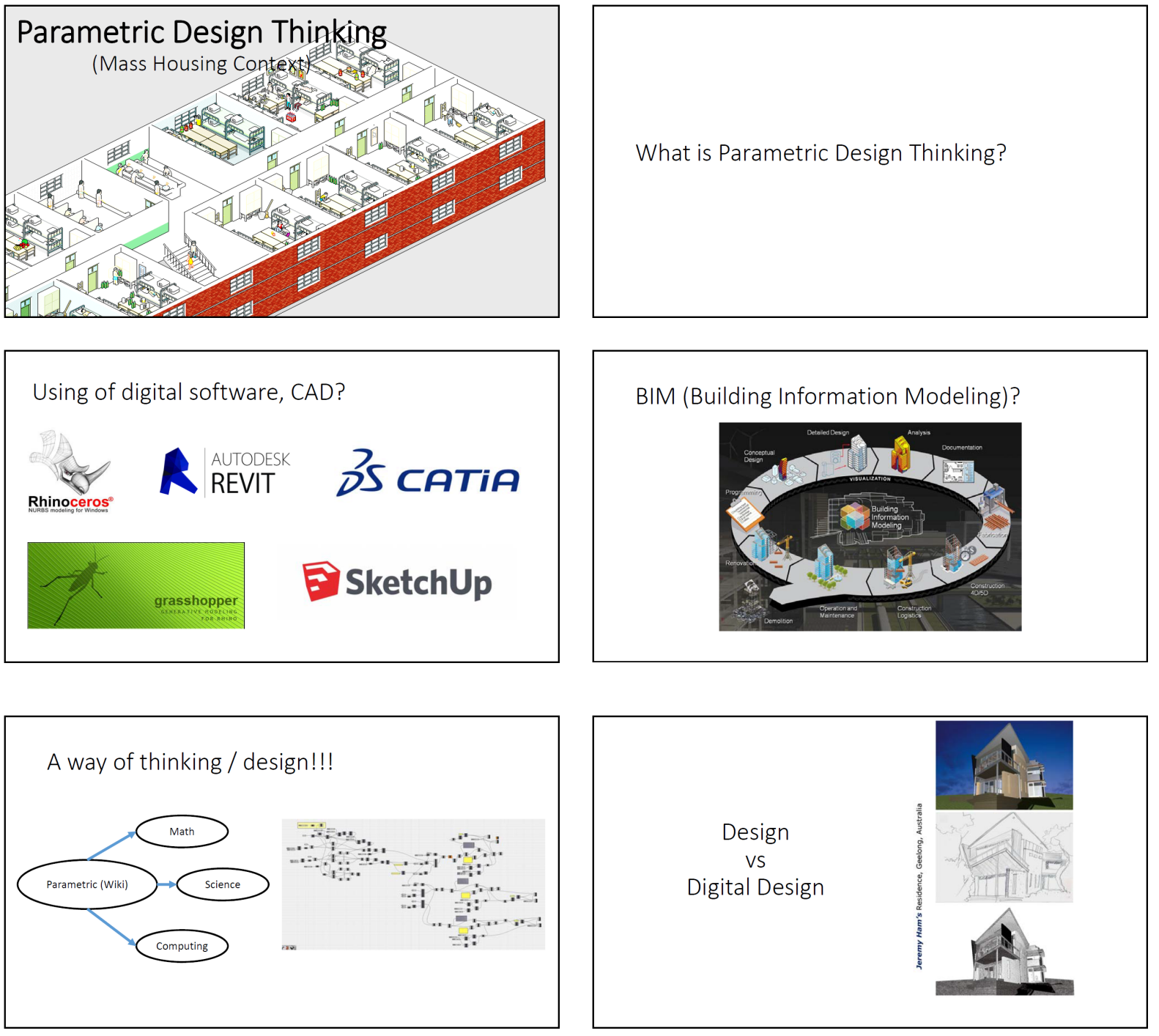

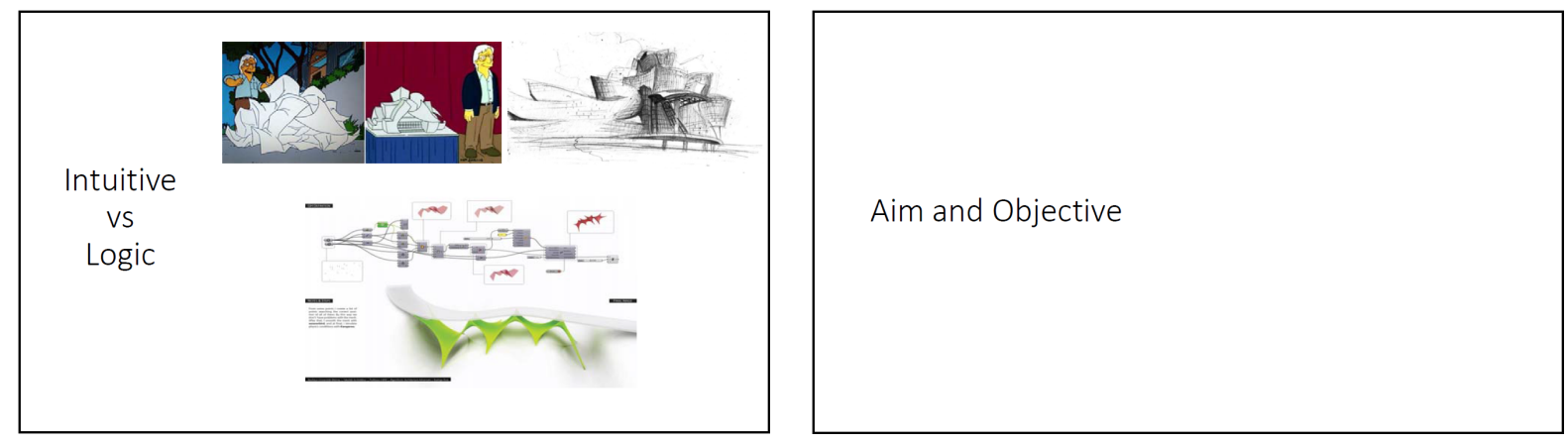

The aim is to impart parametric design thinking and development strategies to the students.

The objective is for the participants to be able to develop a set of parameters and rules for a building design (mass housing) AND for the coordinator to understand and explore the various possibilities of this strategy.

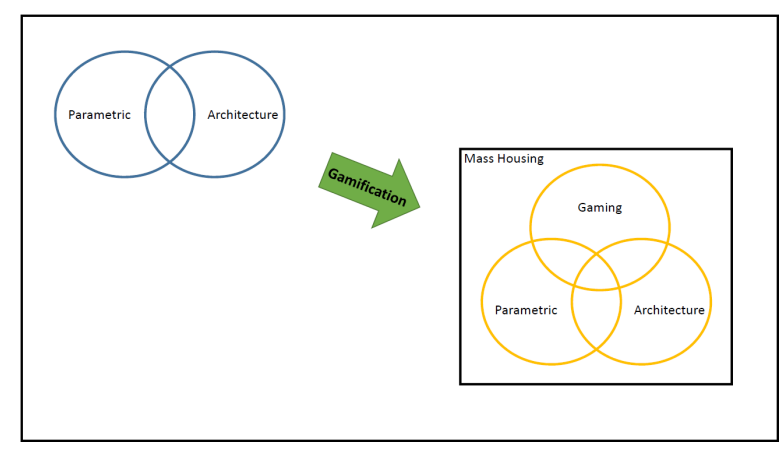

What is Gamification?

Gamification is the use of game thinking and game mechanics in non-game contexts to engage users in solving Gamification is the
problems. (Wiki)

Why Gamification and How it is related to Parametric architecture?

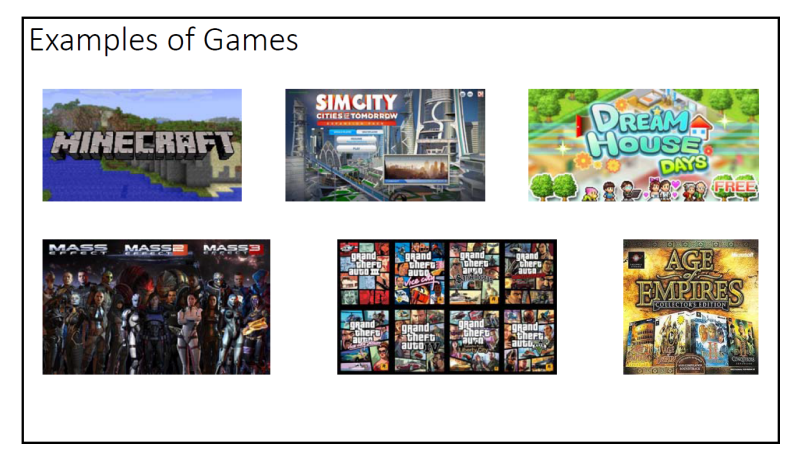

Characteristics of Games
- Rule-based
- Narrative
- Open-ended
- Freedom of Choices within a controlled storyline
- Highly visual-based
- Intuitive operations

\section{Parametric Architecture}

is a design method in which the output - image, sound, architectural models, animation - is generated by a set of rules or an Algorithm, normally by using a computer program. Most generative design is based on parametric modeling. It is a fast method of exploring design possibilities that is used in various design fields such as Art, Architecture, Communication Design, and Product Design. Typically, generative design has: - A design schema

-A means of creating variations

- A means of selecting desirable outcomes

Phase 1

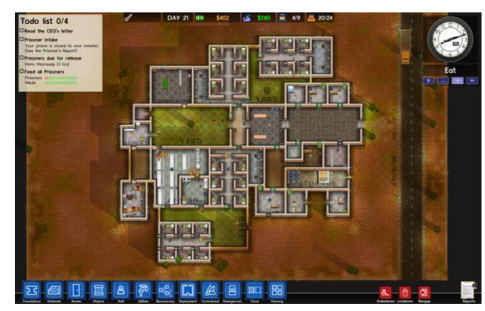

Design a prison of your own Follow the instruction and ulfil each requirement Unlock all elements Make a power-point or video and report the pros and cons of the systems. Explain some of the difficulties you faced during Comments on the relationship with architecture design
procress.

\section{Phase 2}

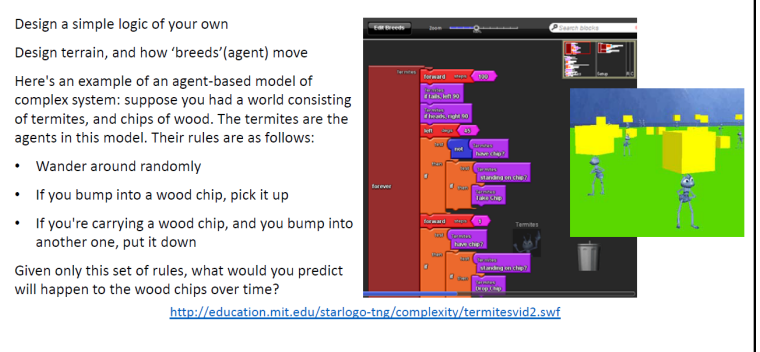



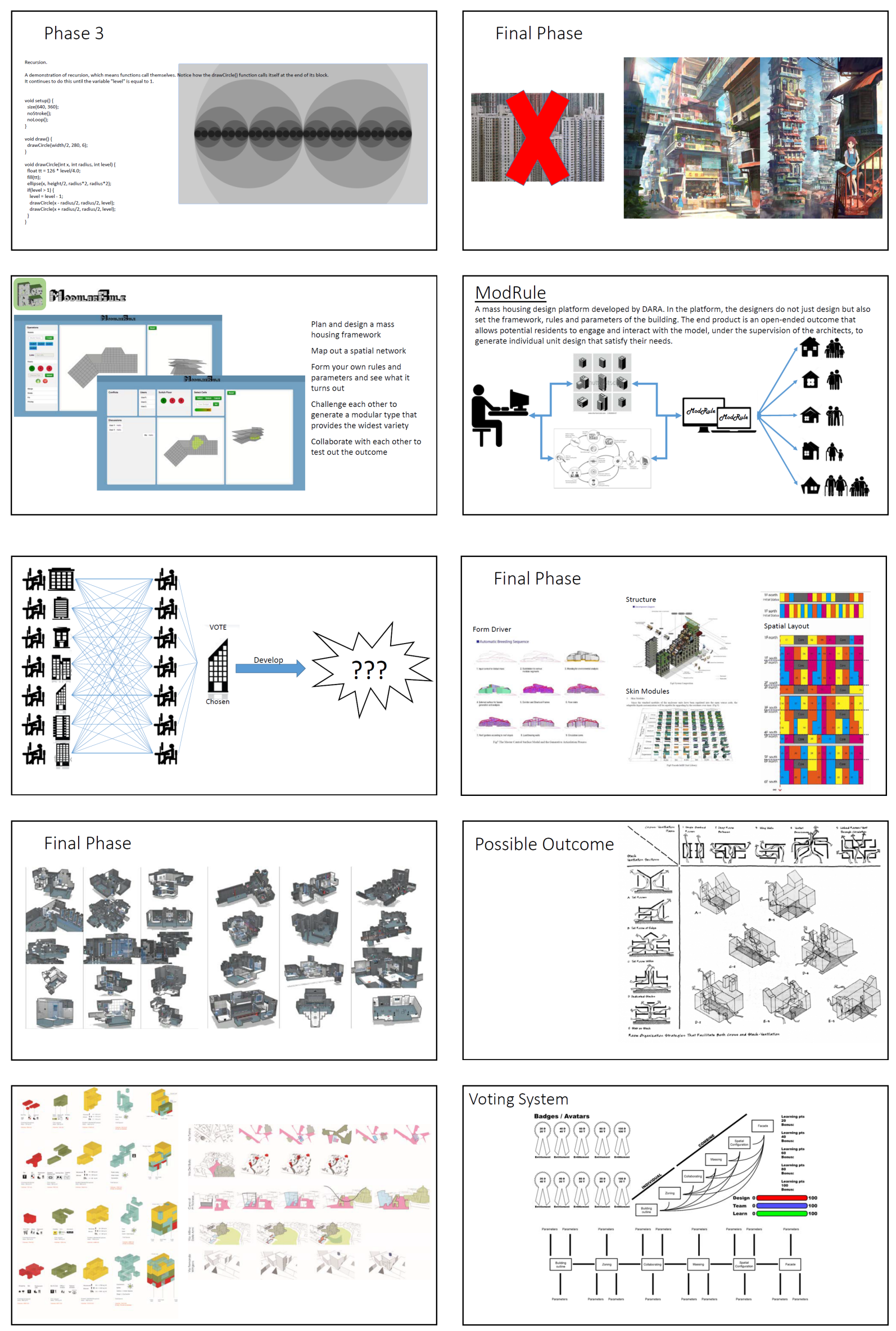


\section{Social media group}

This is a Facebook group created to communicate with the participants. This can be found in https://www.facebook.com/groups/digitaldesignthinking/.

1. Introduction page of the Facebook group with its participants.

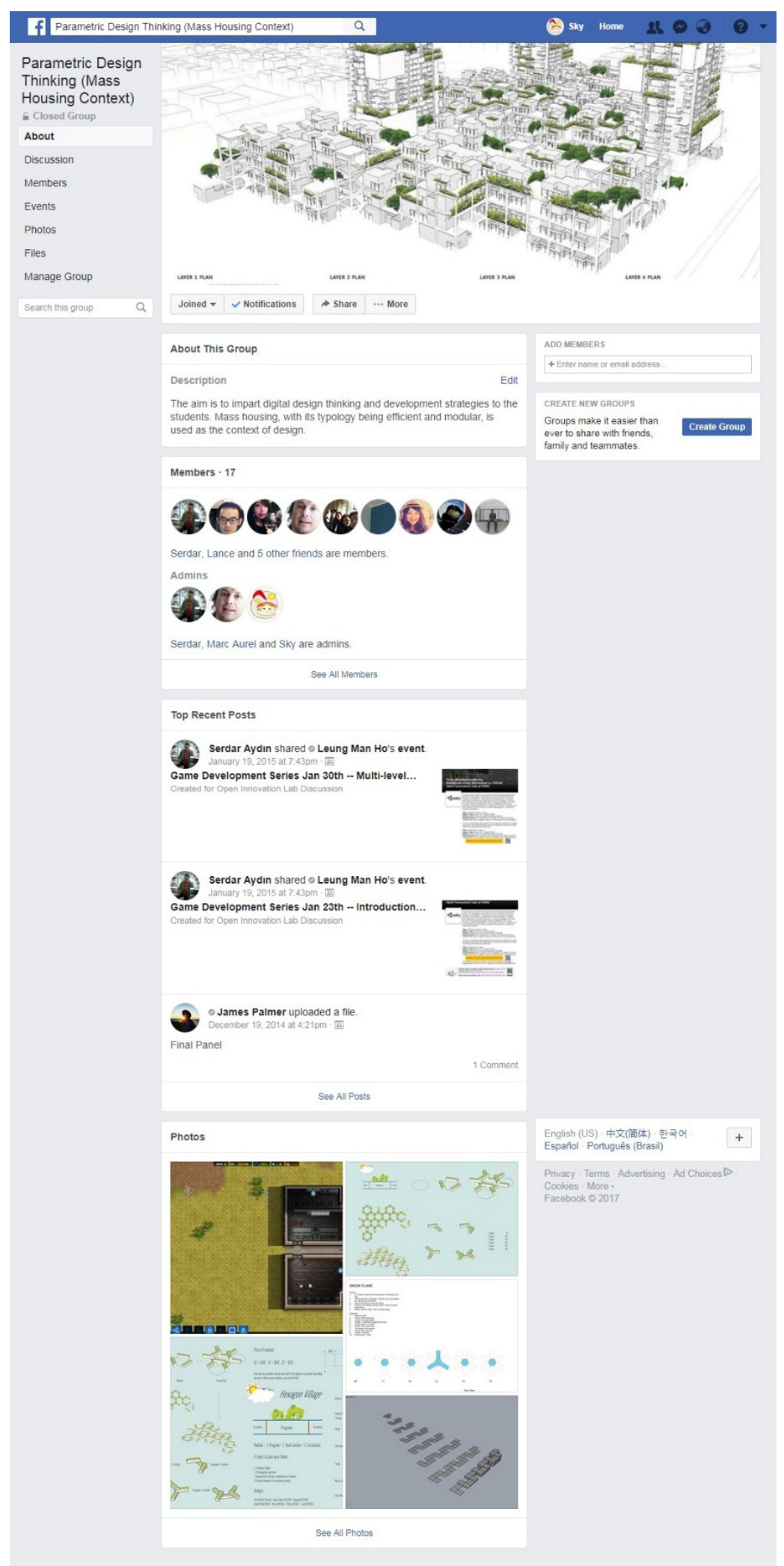


2. Comments of the participants after trying out gamification. The discussions on social media show how useful an online platform mediates design collaboration.

\section{B. Ashitaka Lin \\ September 16, 2014}

This is not final result, but I will stop here. Prisoner architect reminded me another game Simcity. I couldn't stop spending a lot of time on it many years ago.Both of them are very interesting. Although none of them will make us a real master of city planning or architecture now, they provide us a platform to test and practice our design in a virtual world. We have seem simulation were implied in many other industries, and recently I just learned some real projects using BIM system to help design by simulating energy consumption. I believe that game/simulation will become more and more powerful and useful. Maybe in a near future, playing a sophisticate SimCity/Prisonararchitect will be an important part of design process.

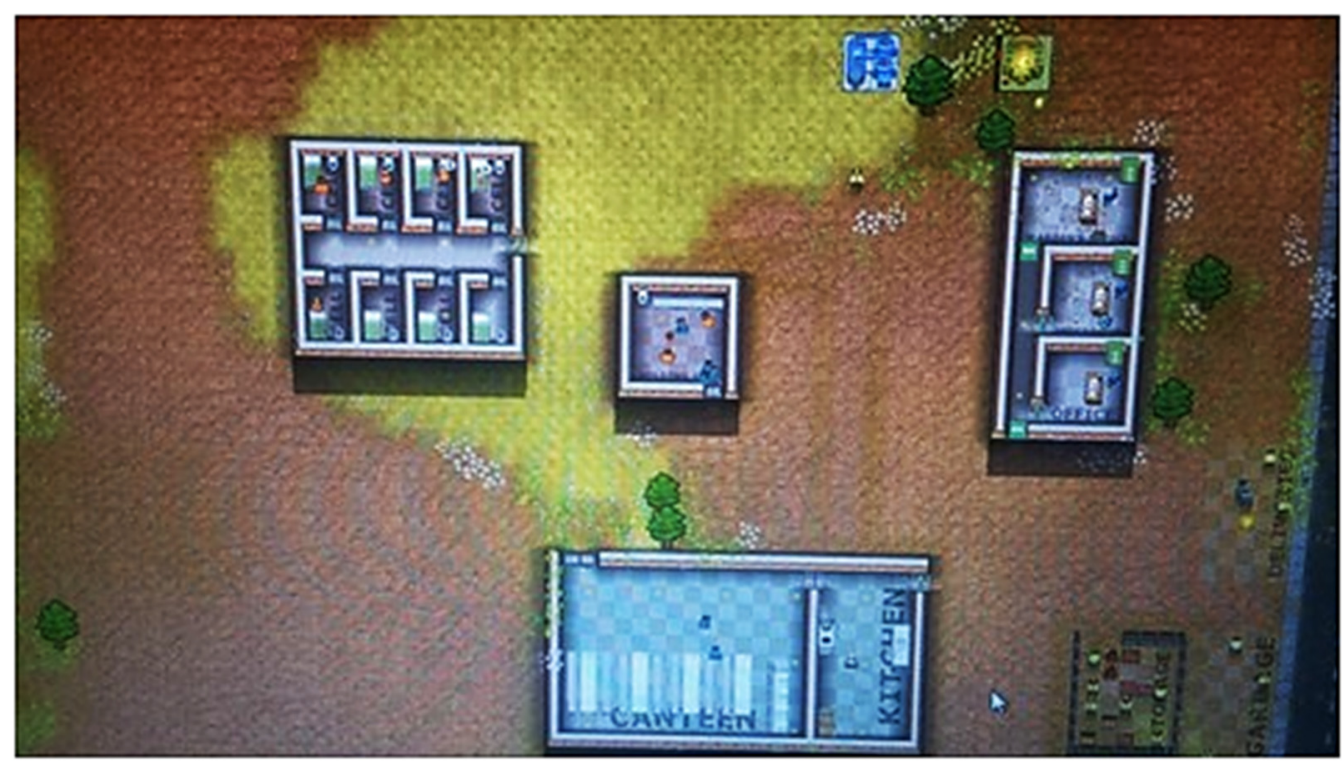

\section{¿ Like Comment}

Sky Lo Yes, the fact that games are more captivating, many are trying to integrate games and architecture to make it become part of the design process with simulation etc. Very interesting plan, your prison has so much freedom!!!! haha

September 16, 2014 at 3:32pm - Like - 1 


\section{Raymond Lee}

September 14, 2014

I played the introduction and created a prison. In my opinion, the approach to create a prison in that game is far from what we design architecture in general practice. What the game concerns is the feasibility of space such whether incorporated with electricity, water supply. However, what I usually concerns in architecture is the spatial arrangement or quality. The prison that I created in the game would be analyzed immediately by the system so that I can see what I am missing or do anything wrong. In real world, I have to point out the problem by my observation.It might be the different between the parametric design approach and general practice.

In term of a game player, this game is much more difficult compared with SIM City because it is not easy to make the space feasible. In other words, it is quite ruled and controlled.

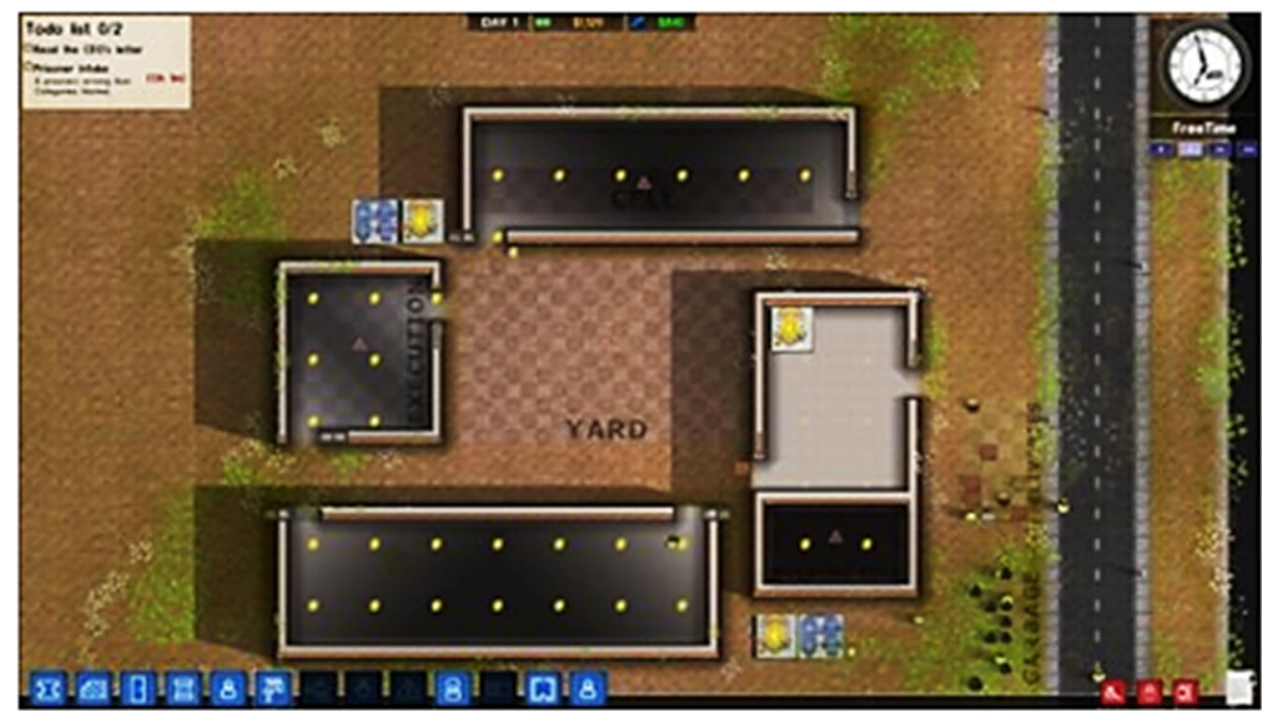

\section{I) Like $\square$ Comment}

Sky Lo

Seen by everyone

View 2 more comments

Raymond Lee As a game player, this is a limitation. It is because I have to spend time to understand all the rules first before I build a prison, which I may lose my interest in this game. Another point of view is that my creativity is bounded by rules. However, as a designer, it is good at understanding the parameters of prison architecture.

September 14, 2014 at 8:57pm - Like

Sky Lo Every game/design has its limitation, every design has to have constrain or there will not be any control. As for creativity bounded by rules, how is that? Too squarish? Maybe you can elaborate a bit on that?

September 15, 2014 at 7:18pm - Like 


\section{Progress of Study}

The progress of the focus group study is recorded through images and screenshot of ModRule taken at different stage of the process.

1. Full outcome of all the design phases by one participants with comments.

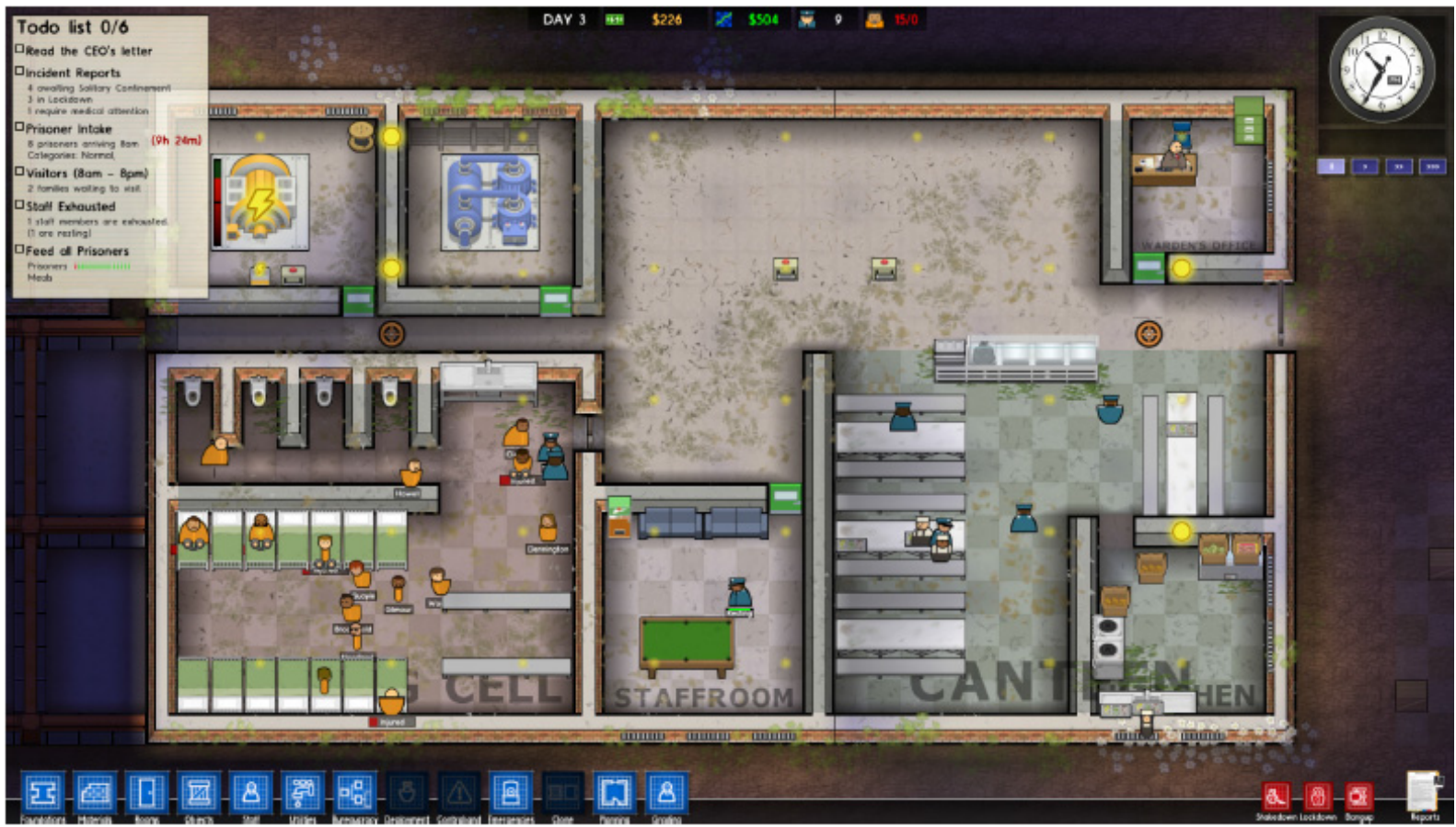

THIS IS THE RESULT OF MY FIRST ATTEMPT ON PRISON ARCHITECT. IT IS RATHER DIFFICULT TO BUILD A PRISON THAT EVERYTHING COULD RUN SMOOTHLY IT IS IMPORTANT TO PLAN AHEAD AND CONSIDER DIFFERENT ASPECTS THOROUGHLY. SUCH AS ELECTRICITY. WATER SUPPLY. ETC. MONEY IS ALSO A LIMITING FACTOR THAT MUST BE CONSIDERED IN ORDER TO KEEP THE PRISON RUNNING AND GROW ING. MORE FACILITIES AND FEATURES ARE UNLOCKED AS THE GAME GOES ON. AND IT GETS MORE SOPHISTICATED AND INTERESTING TO PLAN AND BUILD THE PRISON.

PRISON ARCHITECT IS DIFFERENT FROM OUR STUDIO PROJECTS IN THE SENSE THAT PRACTICAL THINGS THAT SUPPORT THE BUILDINGS ARE REQUIRE MOST CONCERN. WHILE THESE ARE THE THINGS THAT WE COULD NOT MANAGE TO CON SIDER IN DETAILS IN OUR DESIGN STUDIO. PRISON ARCHITECT PROVIDES A SIMU LATION OF REAL LIFE CONSTRAINTS DURING THE BUILDING PROCESS AND THIS IS SOMETHING THAT WE CAN NOT OVERLOOK IN A REAL PROJECT. 


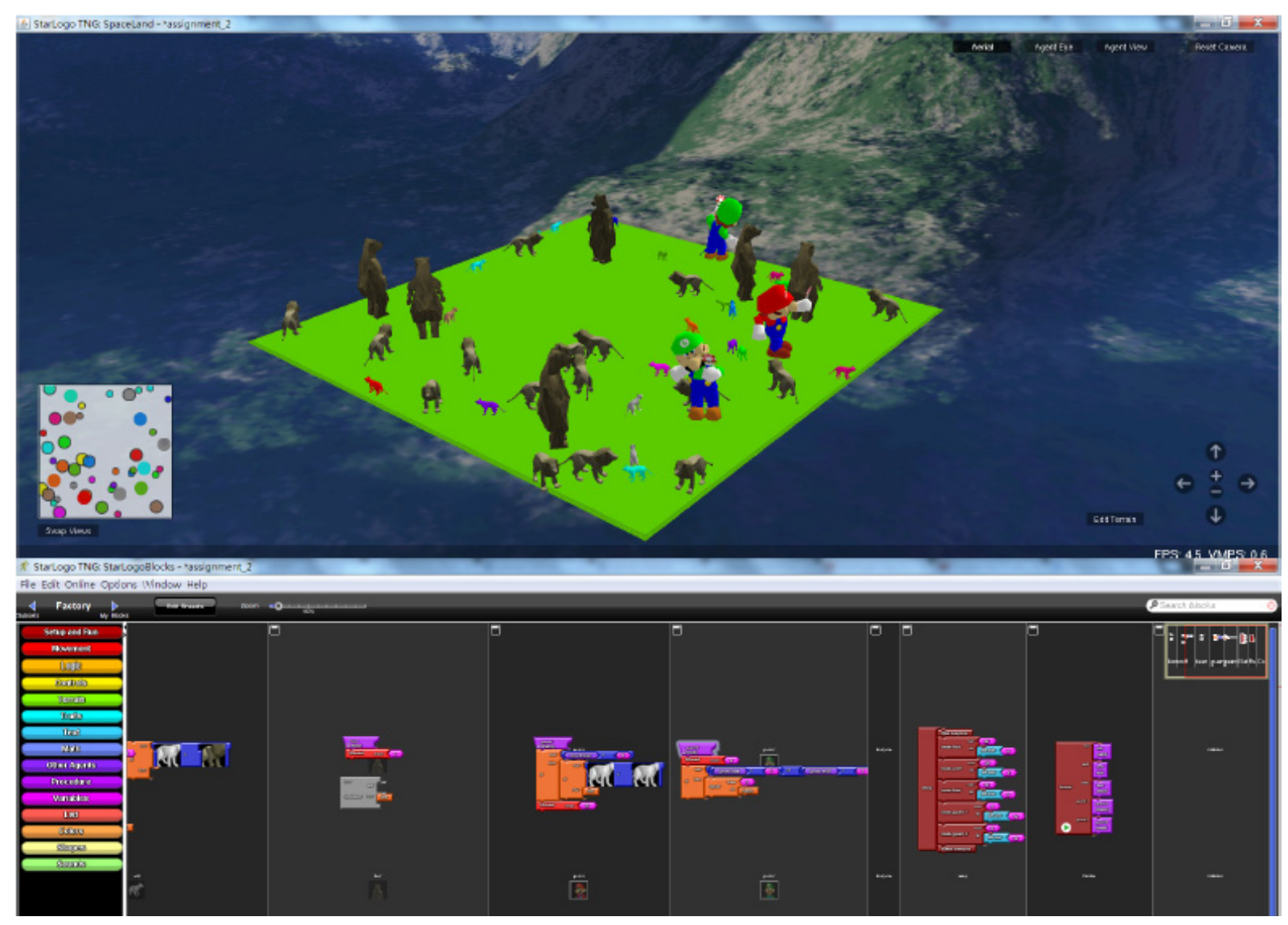

I HAVE SET UP FOUR BREEDS. THEY ARE WOLVES. LIONS. BEARS AND GUARD. MY PROGRAMME TRY TO STIMULATE THE FOOD CHAIN IN NATURE. WHEN WOLF COL LIDES WITH LION. WOLF WOULD DIE. WHEN LION COLLIDES WITH BEARS. LION WOULD DIE. WHEN BEAR COLLIDE WITH WOLF. BEAR WOULD DIE. THESE THREE BREEDS ARE PLAYING THE ROLE IN THE FOOD CHAIN CYCLE. WHEN THE NUMBERS OF ONE OF THE BREEDS DECREASE TO A VERY LOW LEVEL. ONE OF THE GUARD WOULD DESTROY THE DOMINATE BREED AND ONE OF THEM WOULD CREATE THE WEAKER BREED.

FOR ME. IT IS QUITE DIFFICULT TO DUE WITH. BUT WHEN I PLAY THIS GAME. I THINK OF THE POSSIBILITY TO SETTING UP DIFFERENT KINDS OF PARAMETERS AND GIVING THE BREEDS NEW MEANING TO STIMULATE SOME CASES THAT ARE DIFFICULT TO PREDICT. THROUGH OBSERVING THE RUNTIME RESULT. NEW PARAMETERS CAN BE ADDED TO HAVE A BETTER RESULT. 


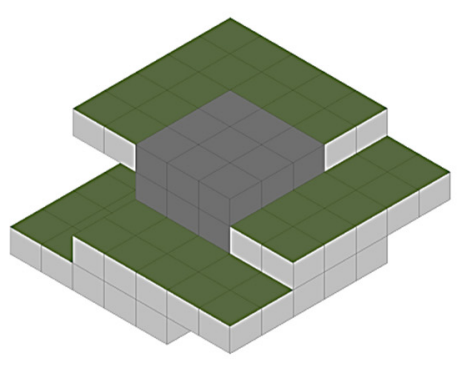

PARAMETER

EACH UNIT IS 3 M $\times 3$ M

UNITS SHADED WITH GRAY COLOUR IS THE PUBLIC SPACE WHICH CANT BE OCCUPIED EVERY FLATS SHOULD AT LEAST OCCUPY 2 DIFFERENT FLOOR

EVERY FLATS SHOULD BE CONTINUOUS

UNIT PRICE : 1/F 20 DOLLARS

2IF 30 DOLLARS

$3 / F 40$ DOLLARS

5/F 60 DOLLARS

WEALTH CHART: EVERY ADULT 80 DOLLARS

EVERY CHILD WOULD GET 40 DOLLARS SUBSIDY

EVERY ELDERLY WOULD GET 60 DOLLARS SUBSIDY

EVERY SINGLE - WOMEN WOULD GET 50 DOLLARS SUBSIDY

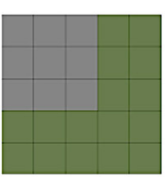

G/F

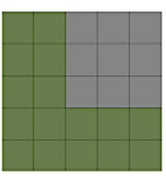

$1 / F$

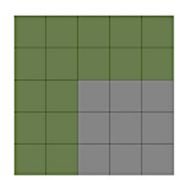

2/F

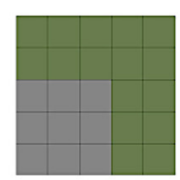

3/F

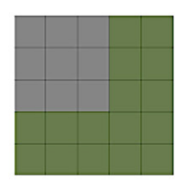

$4 / \mathrm{F}$

FINAL ASSIGNMENT I MASS HOUSING CONTEXT

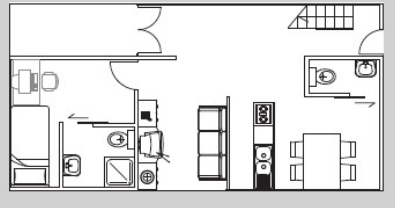

1/F PLAN

$\ominus$

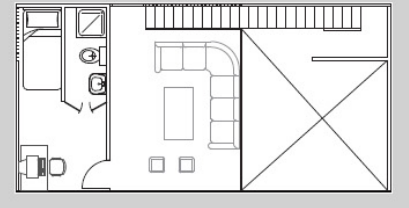

F PLAN
T 1

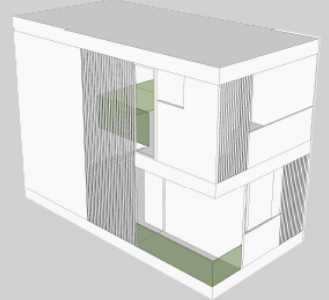

$\Theta$ 
2. Collaboration process using ModRule.

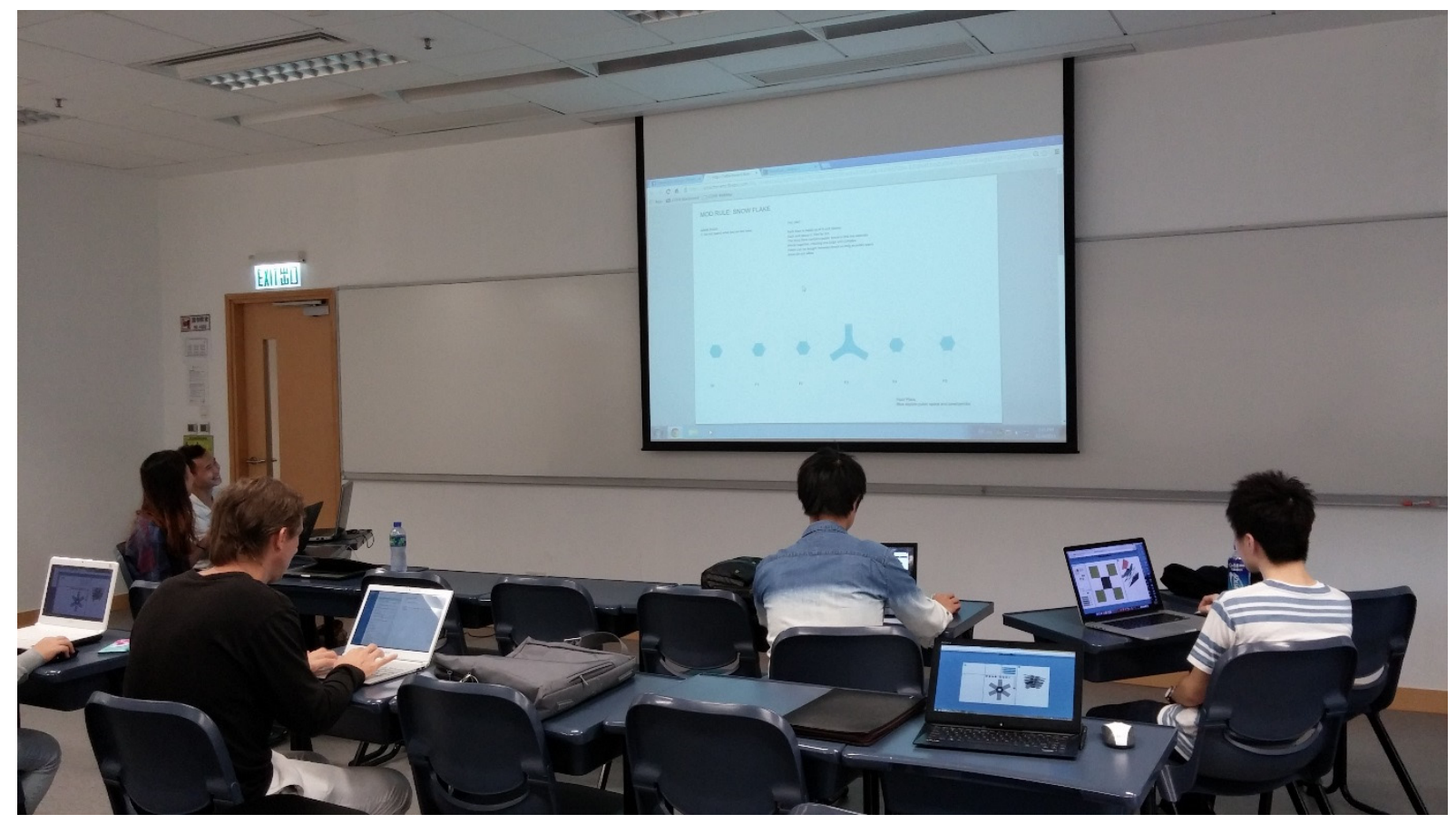

3. Choosing the role to play.

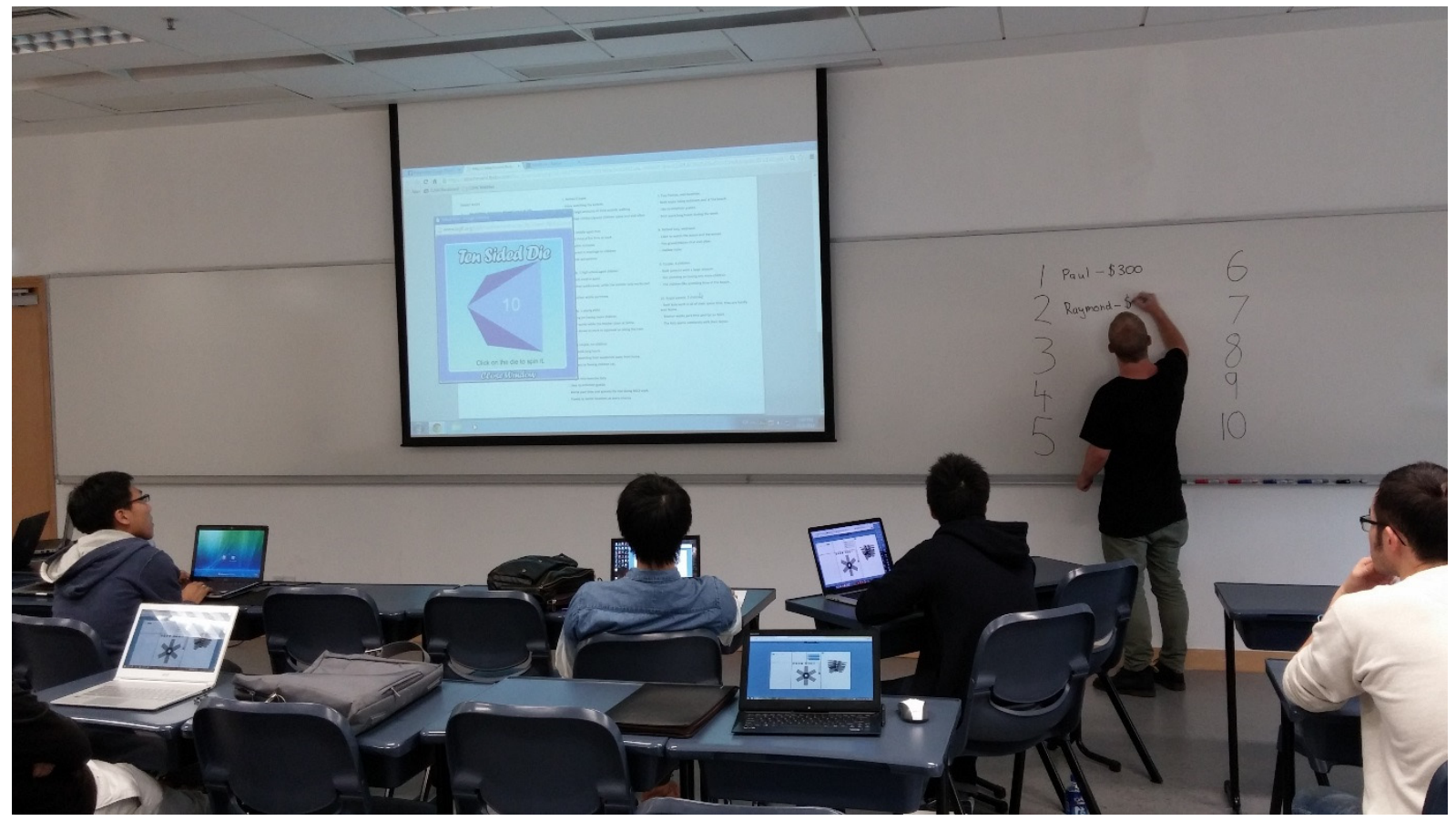


4. Collaborating using one of the design.

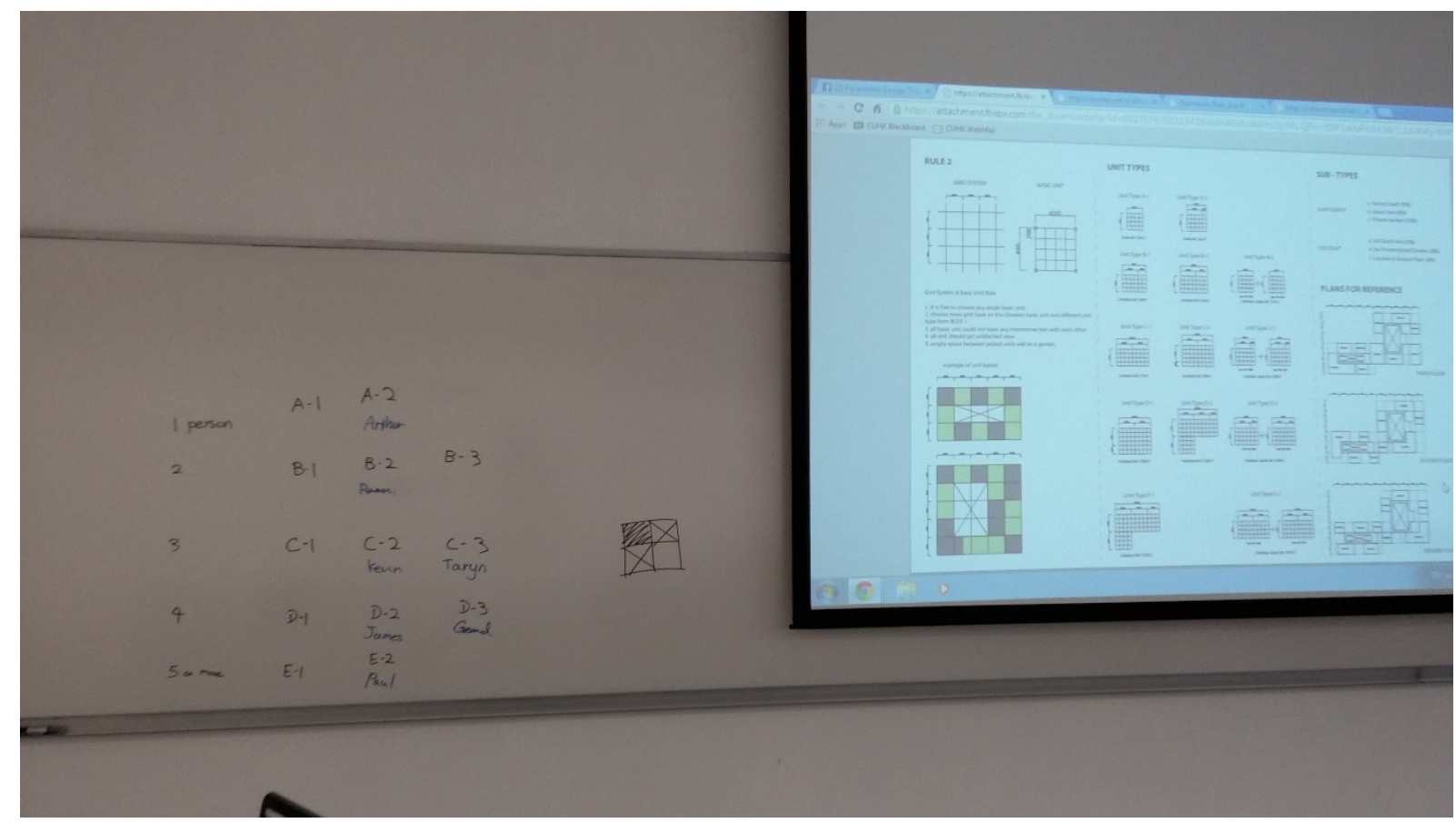

5. Setup of one of the design.

\section{ModRule}

$\frac{\text { ROLES: }}{1, \operatorname{Sin}}$

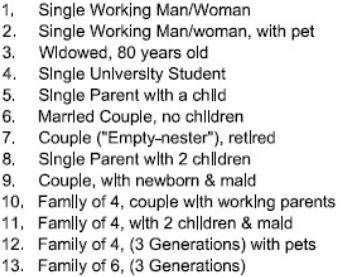

$\$ 150$
$\$ 175$
$\$ 125$
$\$ 100$
$\$ 200$
$\$ 300$
$\$ 400$
$\$ 250$
$\$ 425$
$\$ 700$
$\$ 625$
$\$ 575$
$\$ 800$

Guideline for minimum required living space:

1 person $-25 \mathrm{~m}^{2}$

2 persons $-50 \mathrm{~m}^{2}$

3 persons $-60 \mathrm{~m}^{2}$
4 persons $-75 \mathrm{~m}^{2}$

6 persons $-100 \mathrm{~m}^{2}$

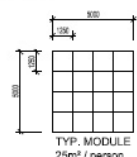

RULES:

1. Create living unit based on chosen role

Must malntaln mln|mum Ilving space as specifleded

. Each unlt must have access to central core

Each unlt must have frontage to exterlor

For famlly with elders and/or pets, unlt must be

adjacent to green space, or located on ground floo

TYPICAL UNIT SIZES (FOR REFERENCE):

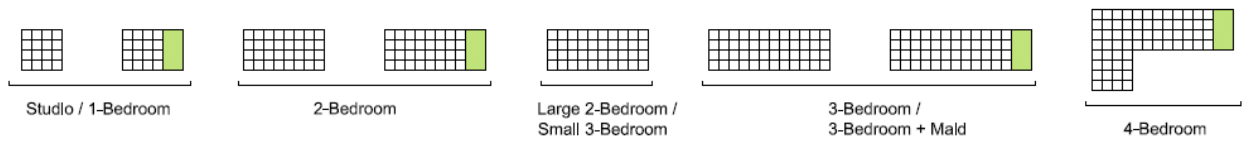

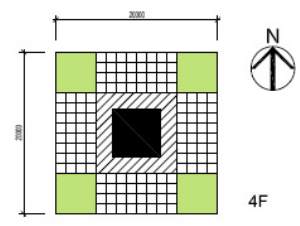
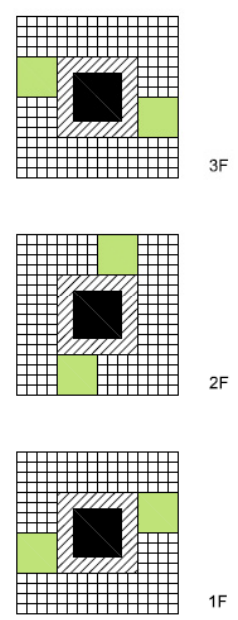

$\stackrel{s x}{+1}$

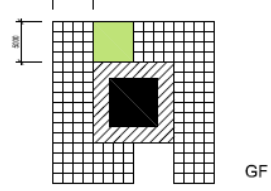


6. Outcome of the collaboration using the nine models created by the nine participants (bottom right was selected to go into details).
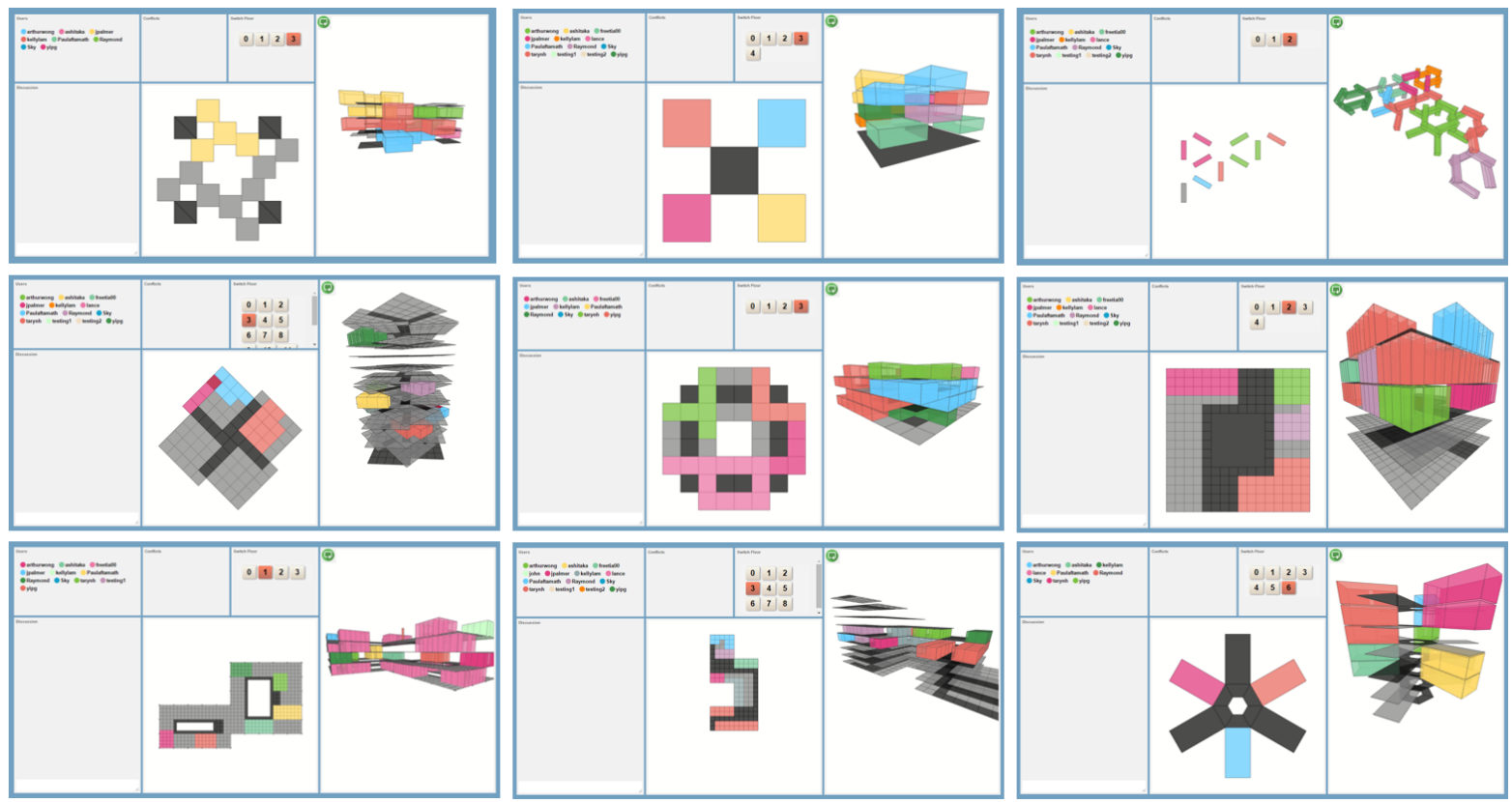

7. The collective final outcome.

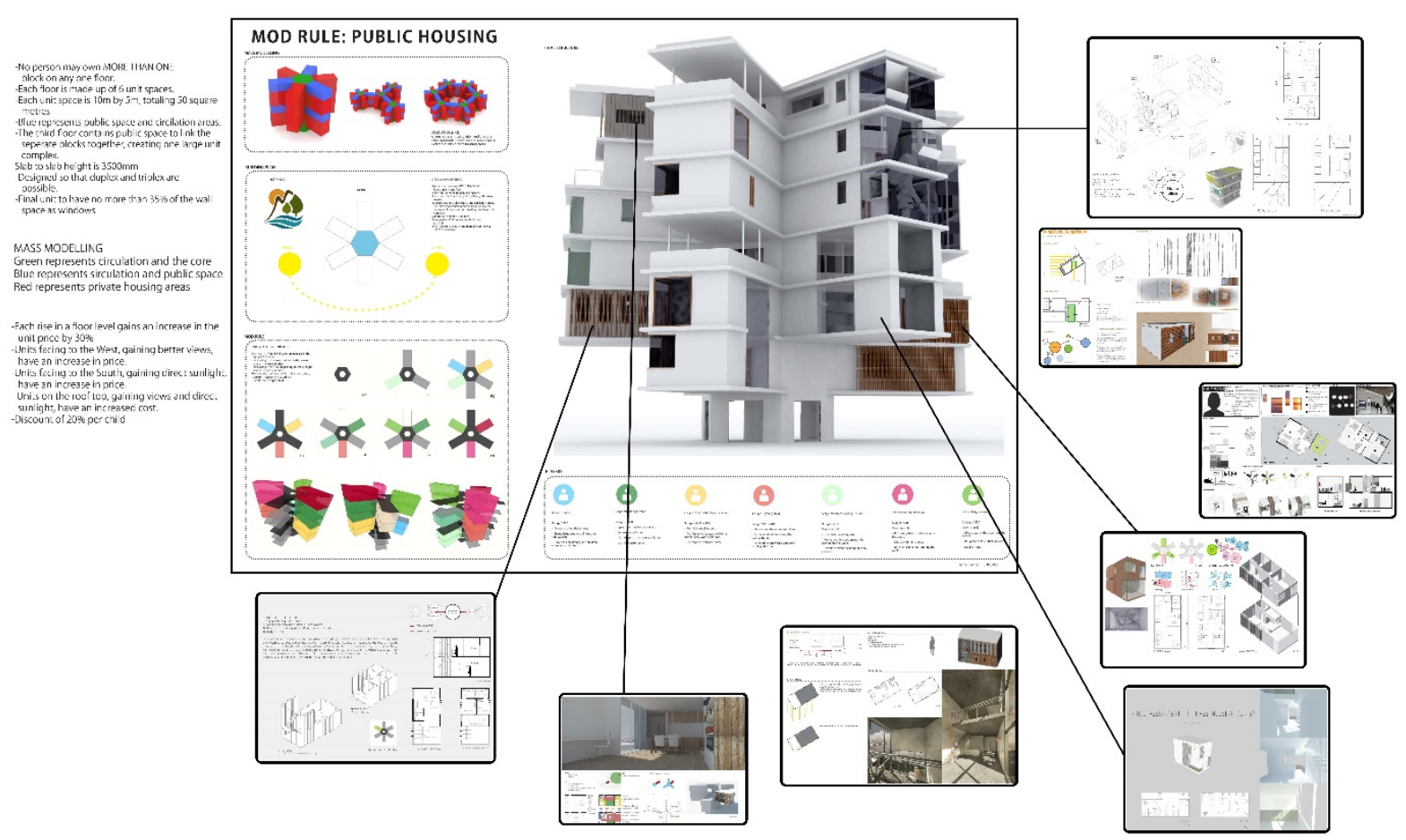


8. Detailed design of each participants with one enlarged.

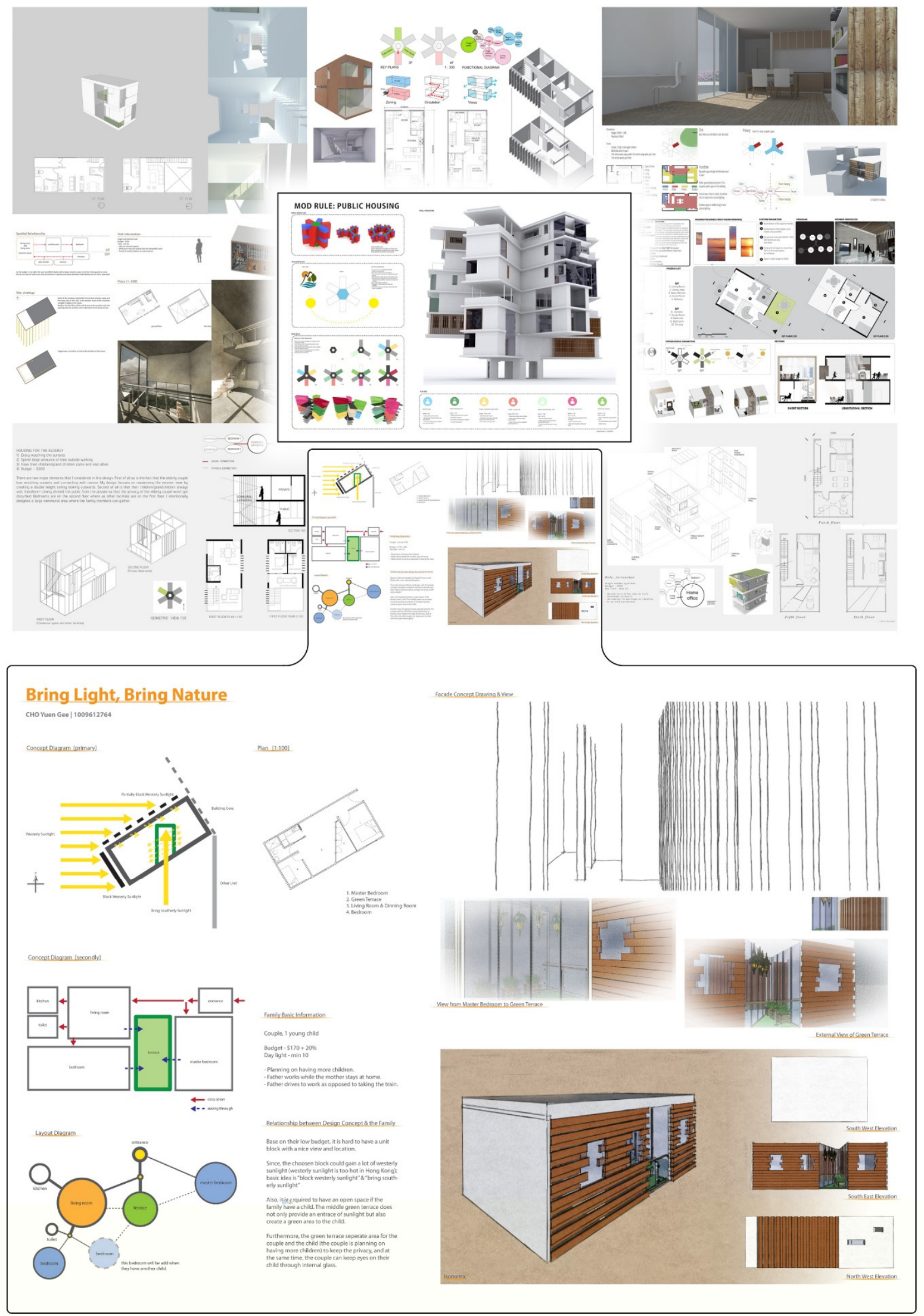




\section{Public Participation}

The public engagement activity using ModRule-Fuzor was held in March 2017.

\section{Publicity of the event}

The activity was published on the website of Te Papa Museum.

MUSEUM OF

NEW ZEALAN

TE PAPA

TONGAREWA

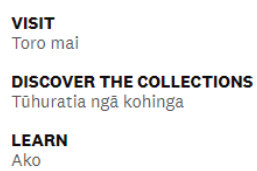

LEARN
AKO
ABOUT

SHOP

Wharehoko

SUPPORT \& JOIN
Search

f

> 10
Open every day $10 \mathrm{am}-6 \mathrm{pm}$

Free entry Free entry xhibitions and activities

\section{Let's Design Together! A Collaborative Housing VR Experience}

By Sky Lo, Prof Marc Aurel Schnabel and Tane Moleta

Victoria University of Wellington School of Architecture

Sky Lo combines 2-D digital design with VR to create a collaborative housing experience. People can design their own living space and communicate with neighbours. The experience aims to create communities through architectural design.

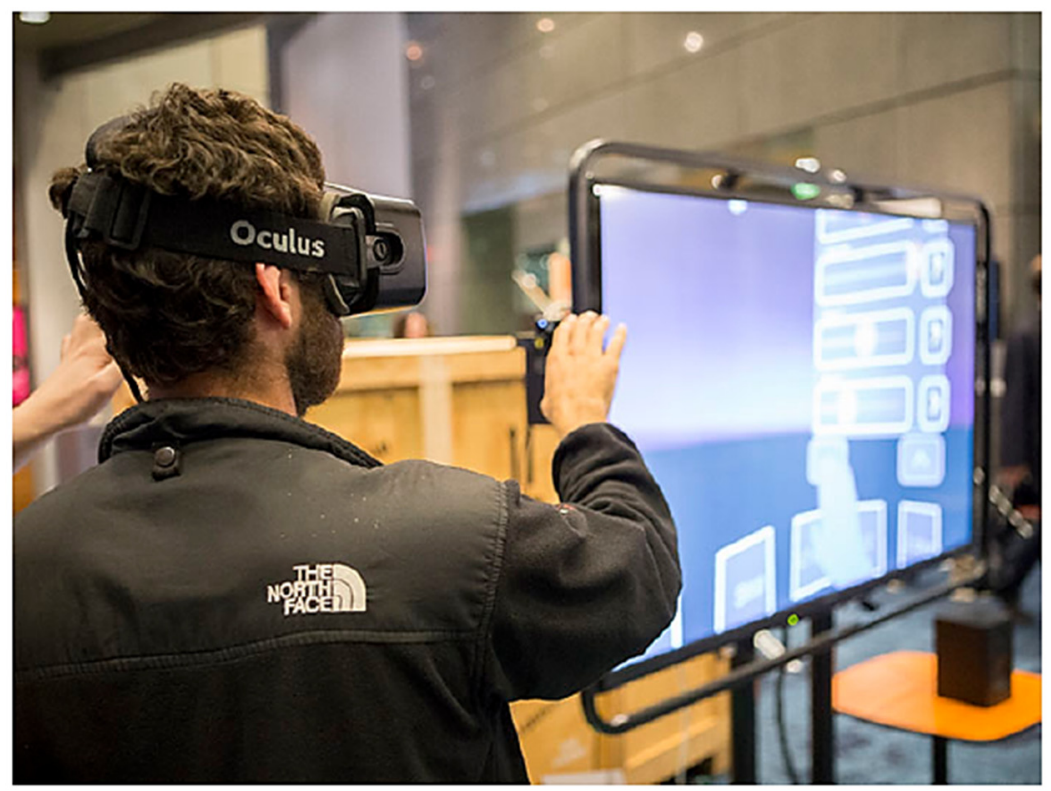

Virtual Reality experience at a Te Papa Talks event, 2016. Photograph by Kate Whitley 
Setup of the hardware and software

1. The setup simulated using digital environment.

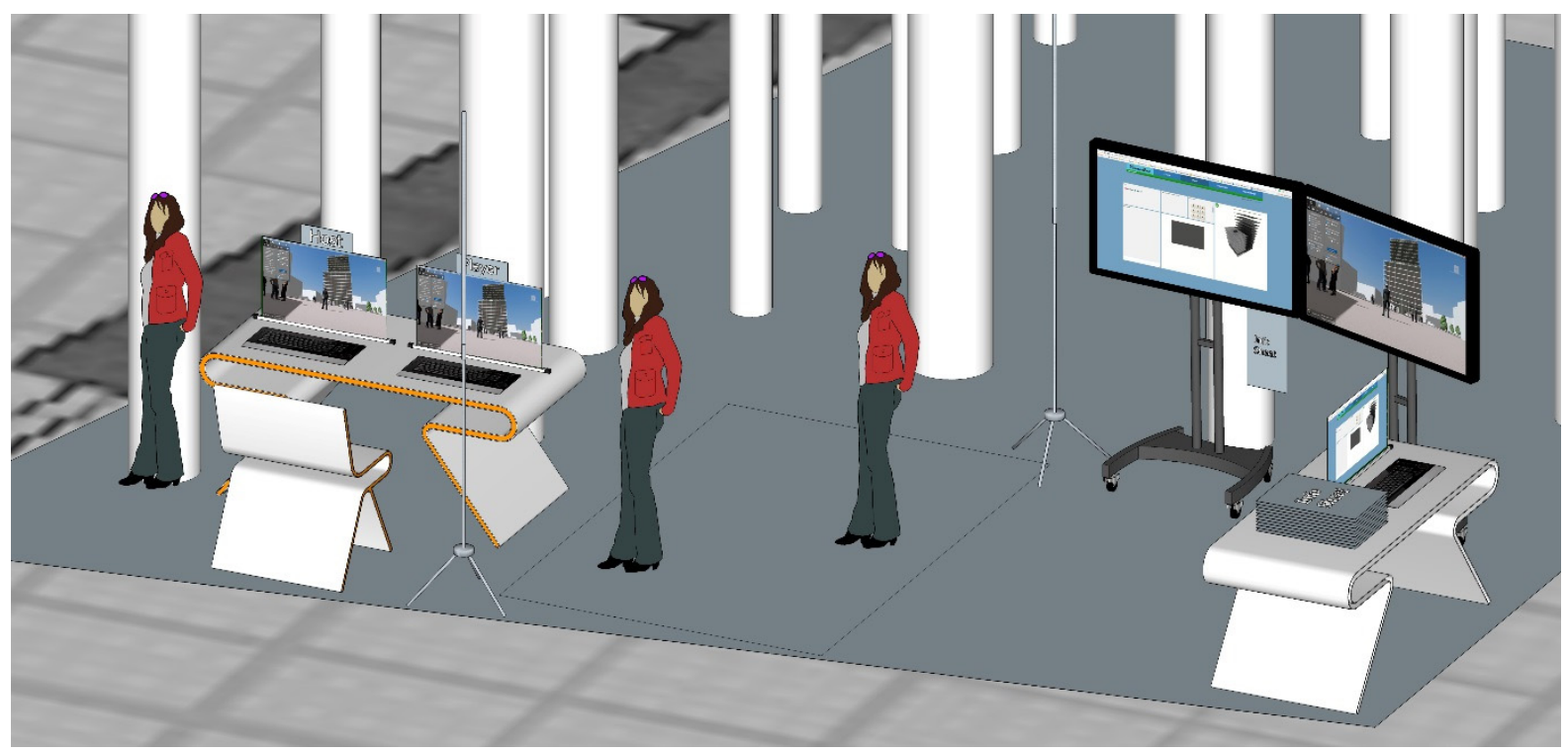

2. The actual setup at the Te Papa museum.

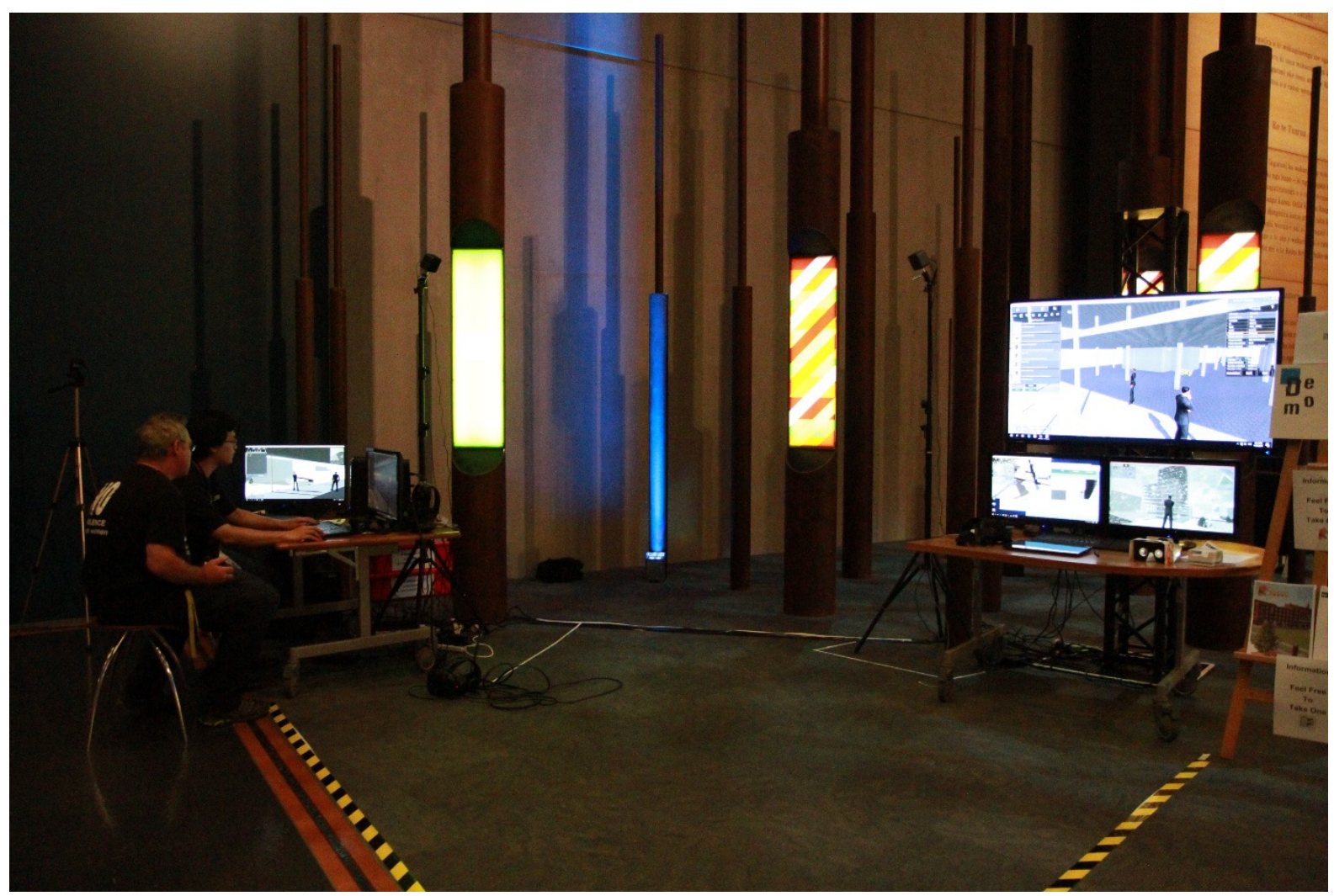


3. Digital model prepared for the design engagement in Fuzor.

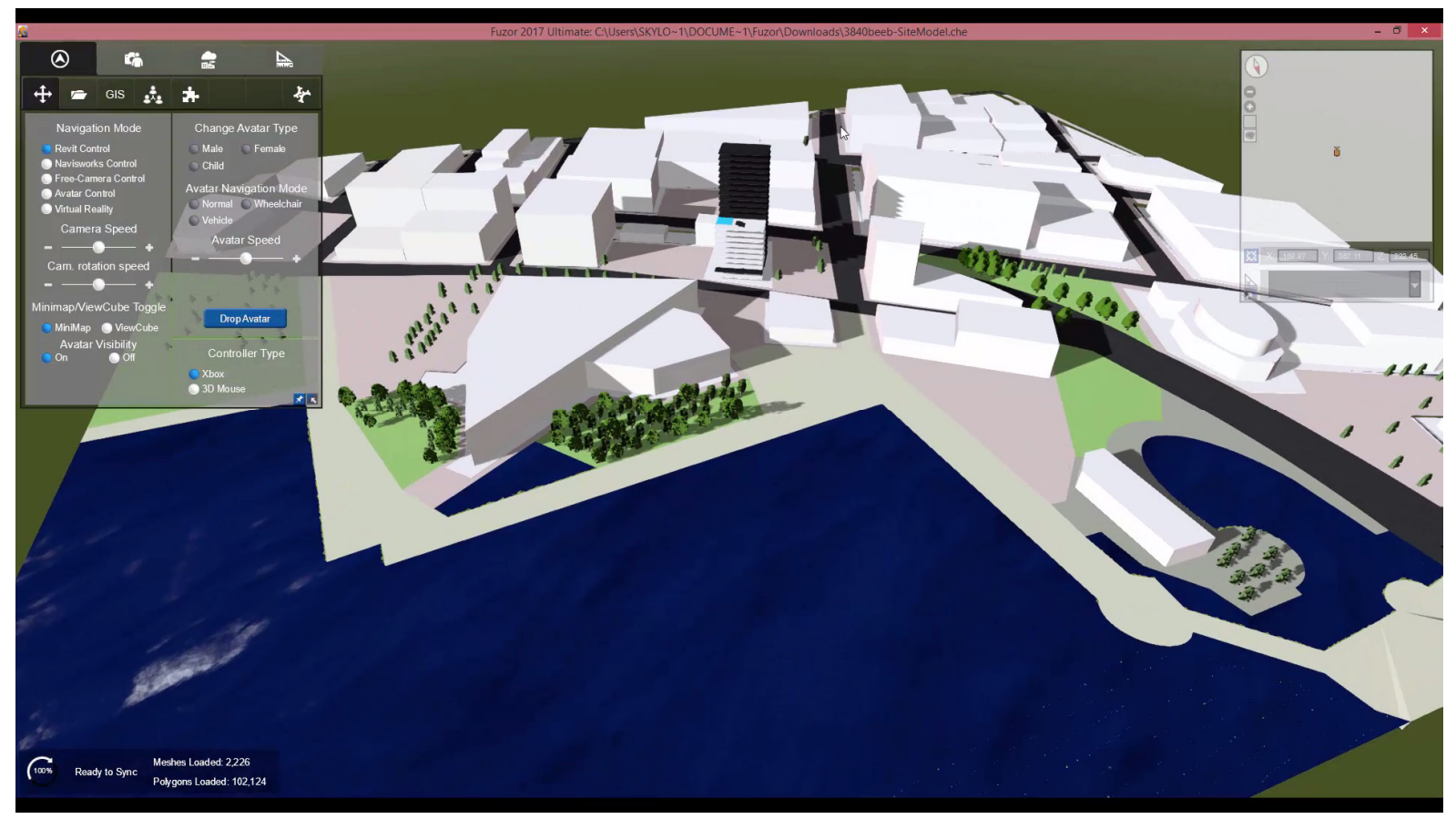

4. Digital model is connected in real-time sync with ModRule input.

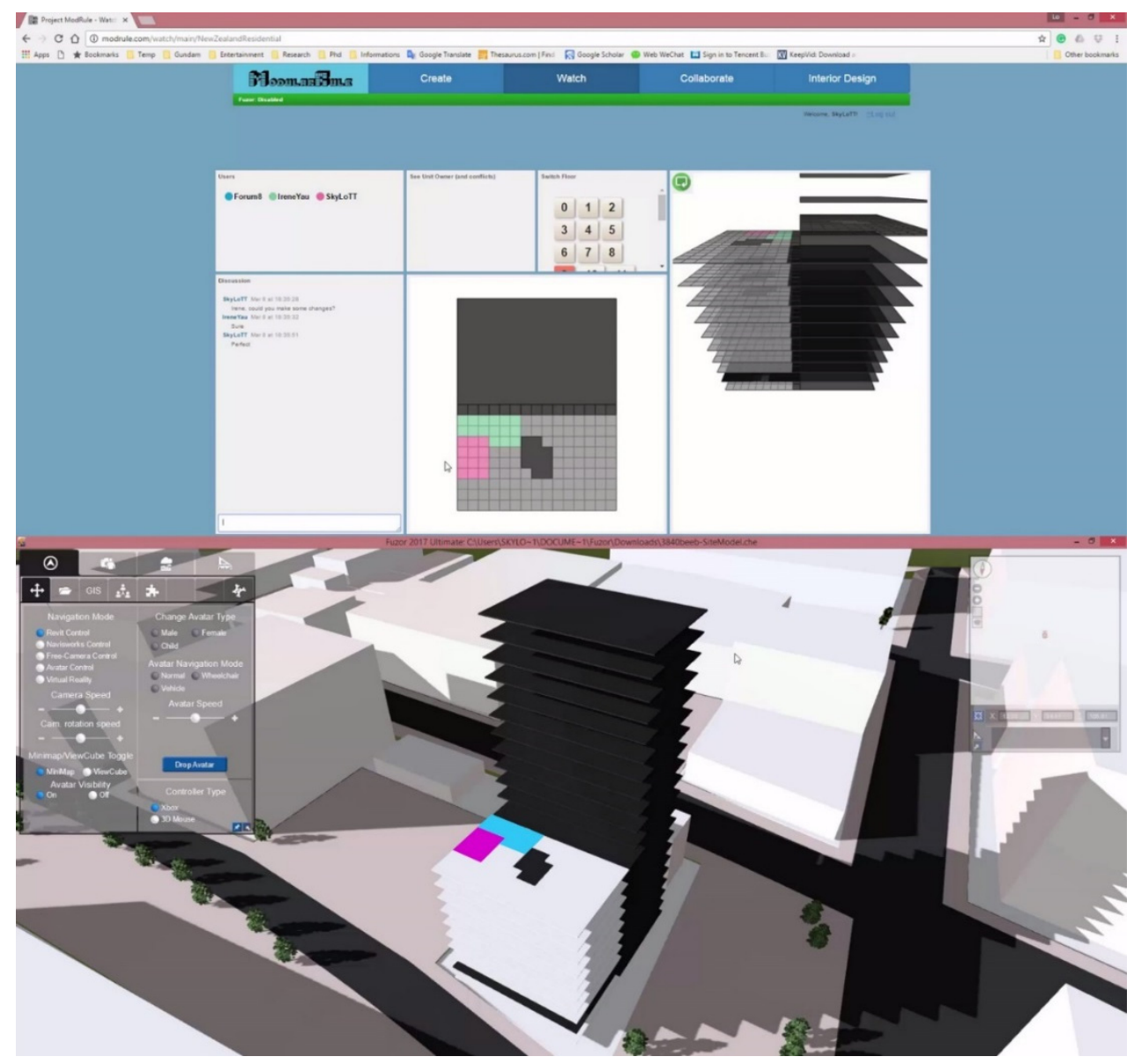




\section{Engaging the Public}

1. Family engaging the design collaboratively using VR tool.

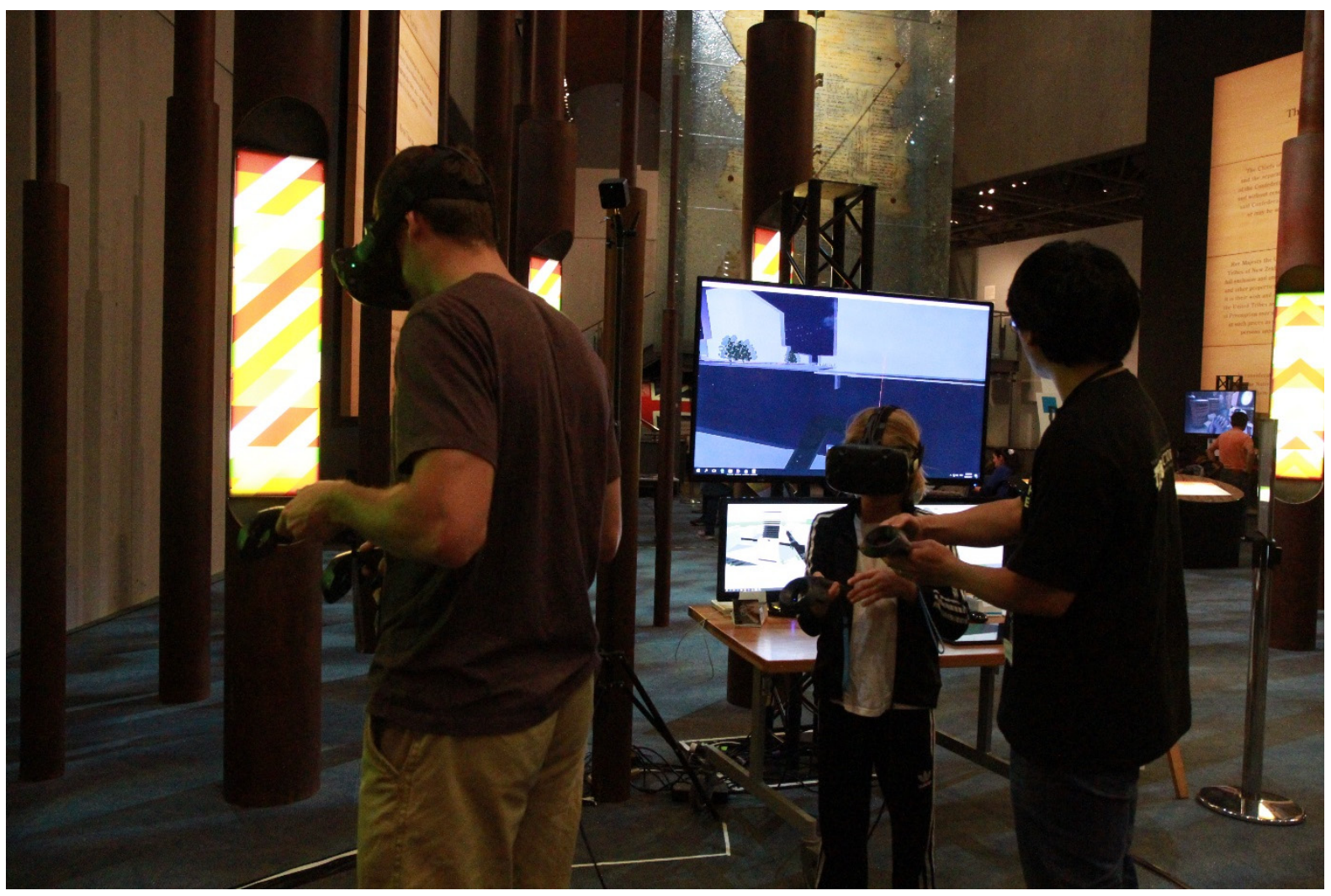

2. Engaging a diverse type of people.

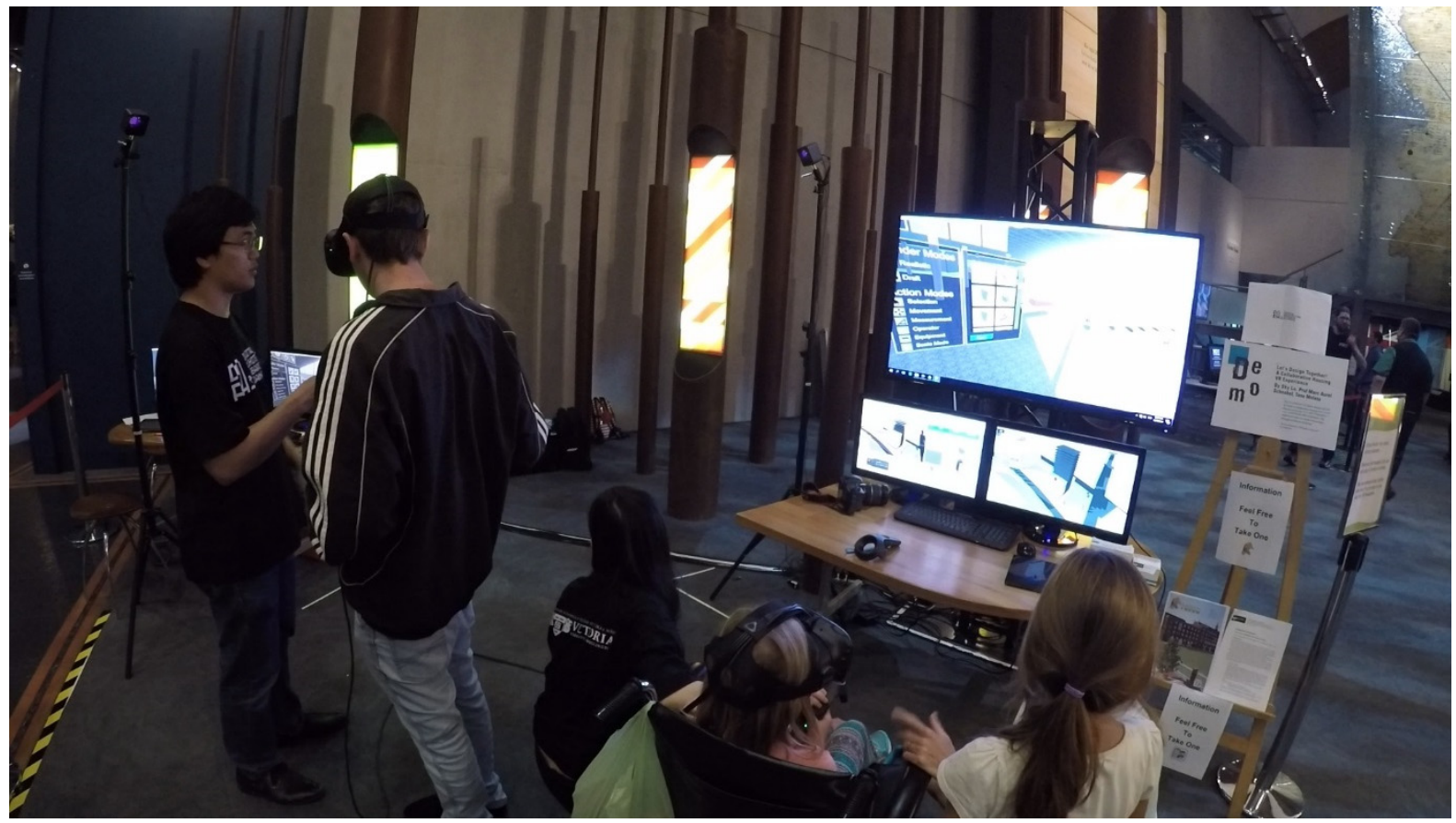




\section{Recorded Outcomes}

1. Moving wall components.

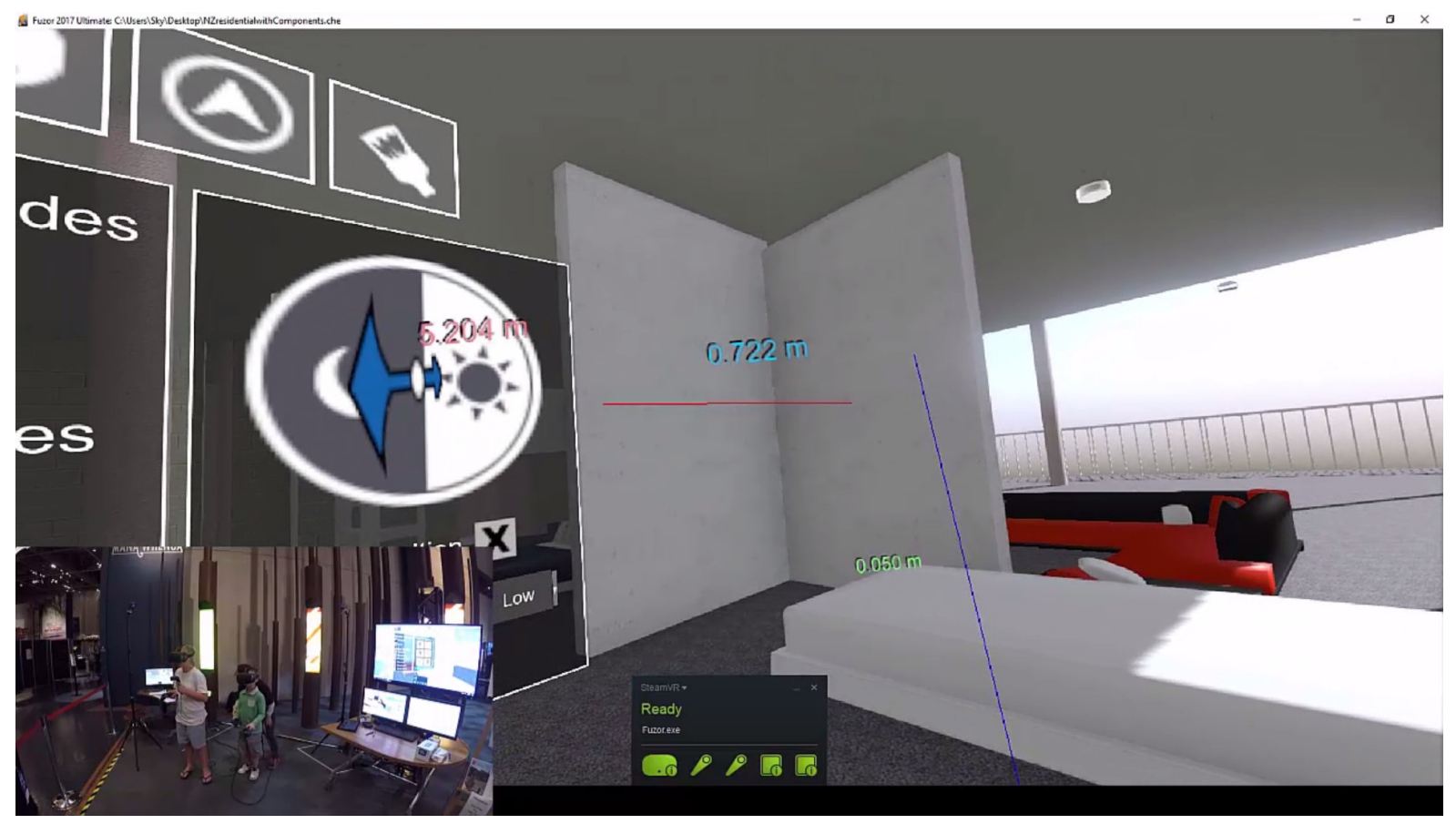

2. Moving furniture.

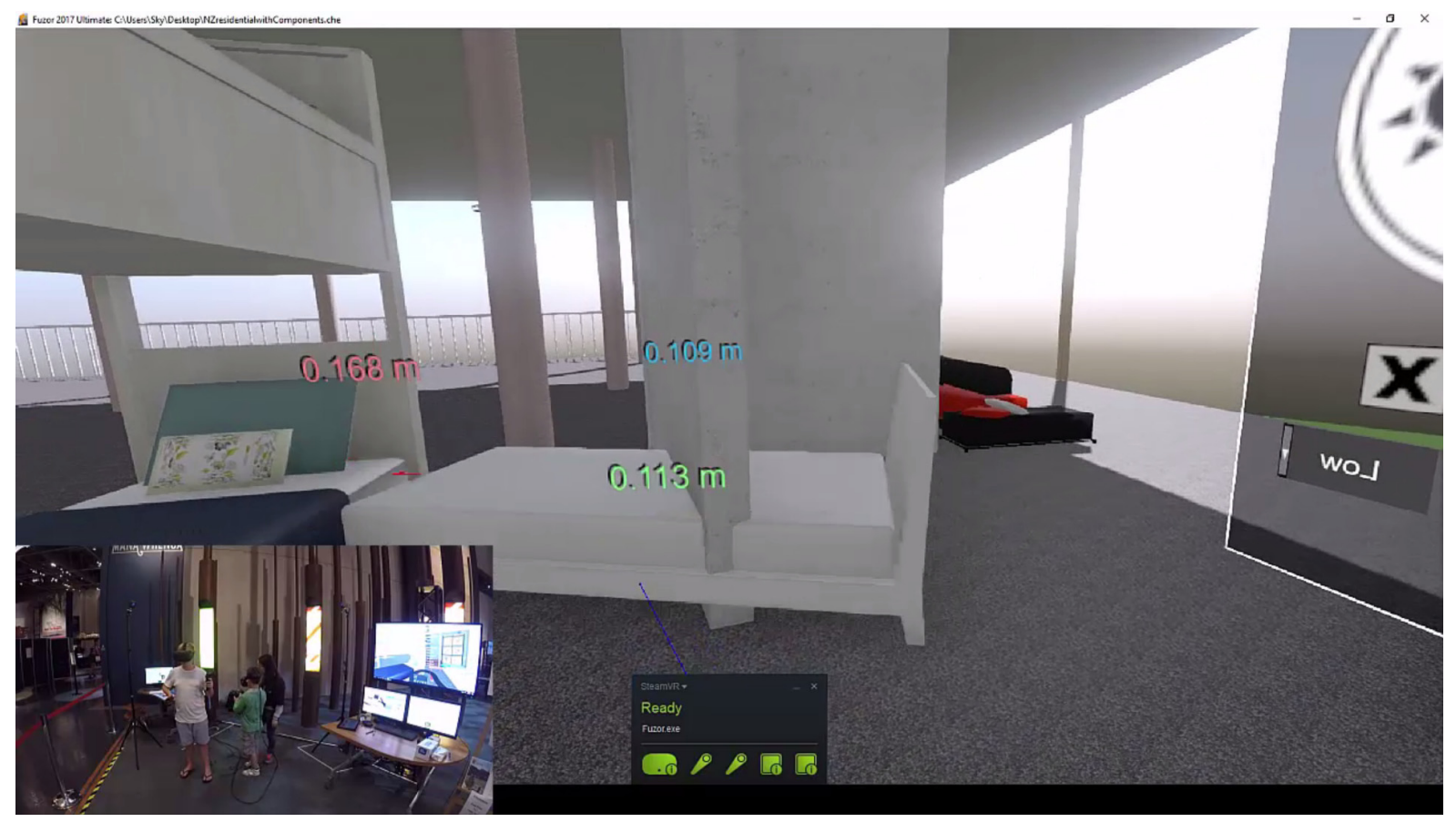




\section{Results}

Two surveys were given to the participants; one before trying out the system, and one after they are done with the design.

1. The 'Pre' survey.

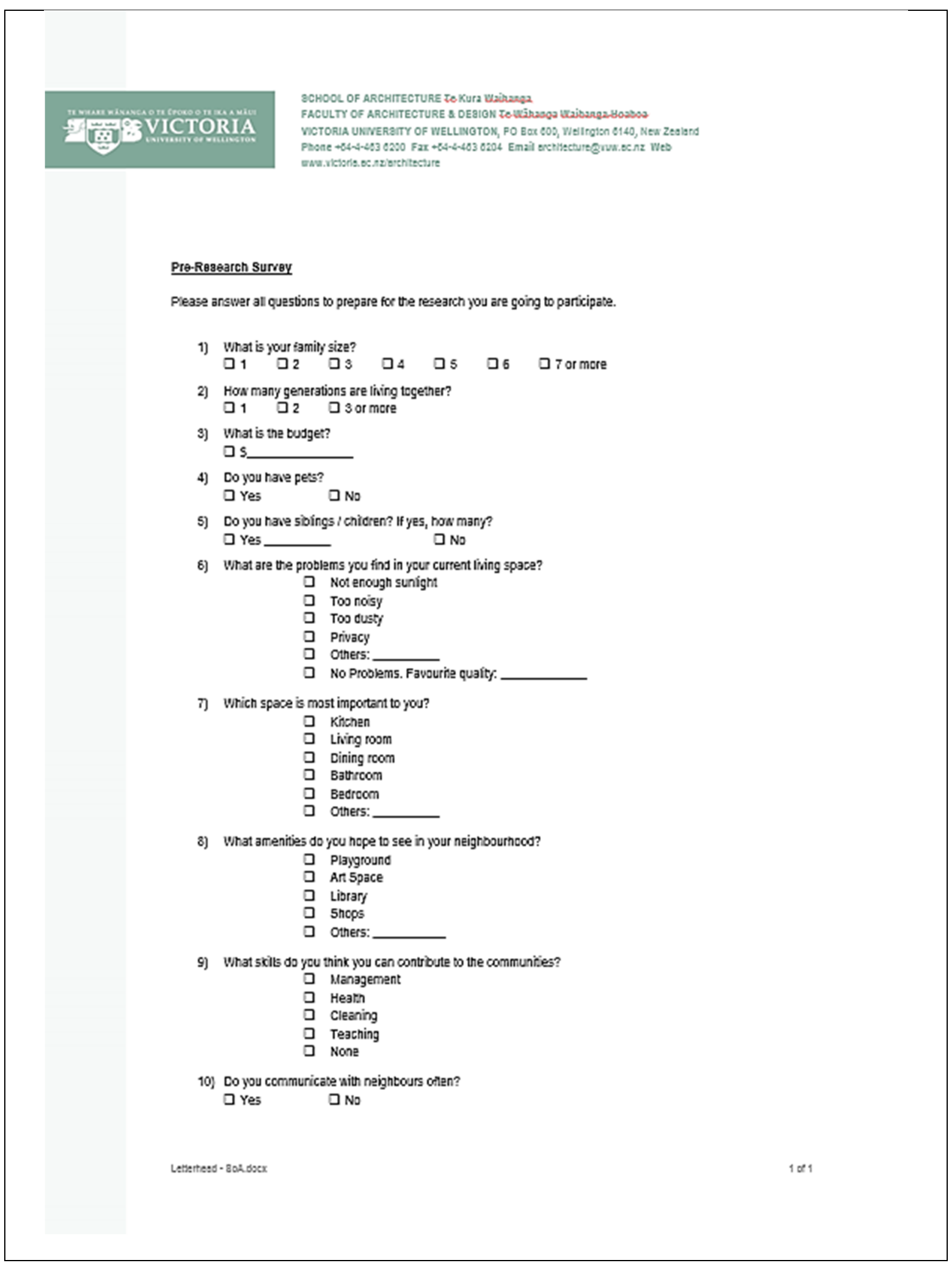


2. Results of the 'Pre' survey (Total 74 participants, including 12 professionals).

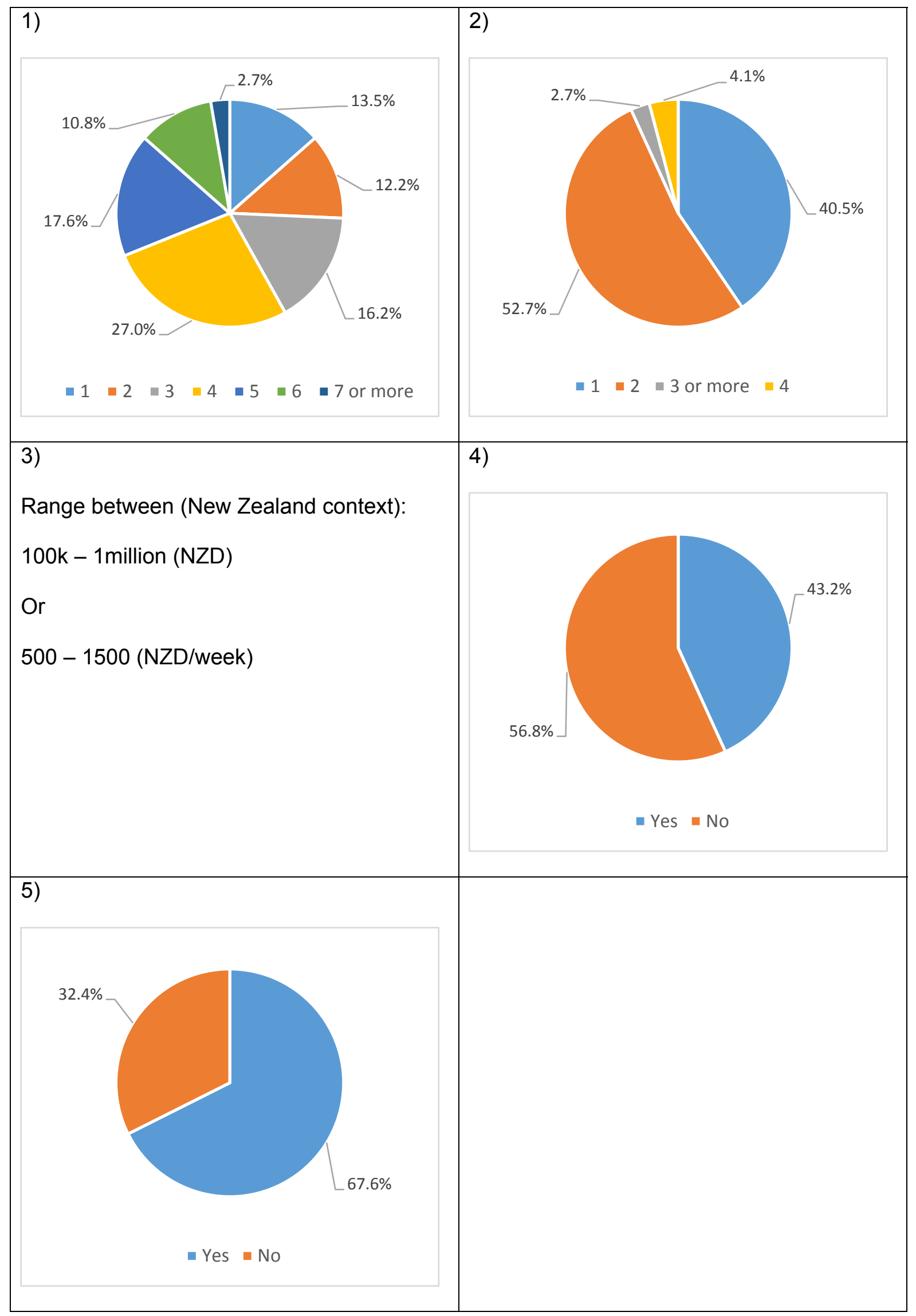




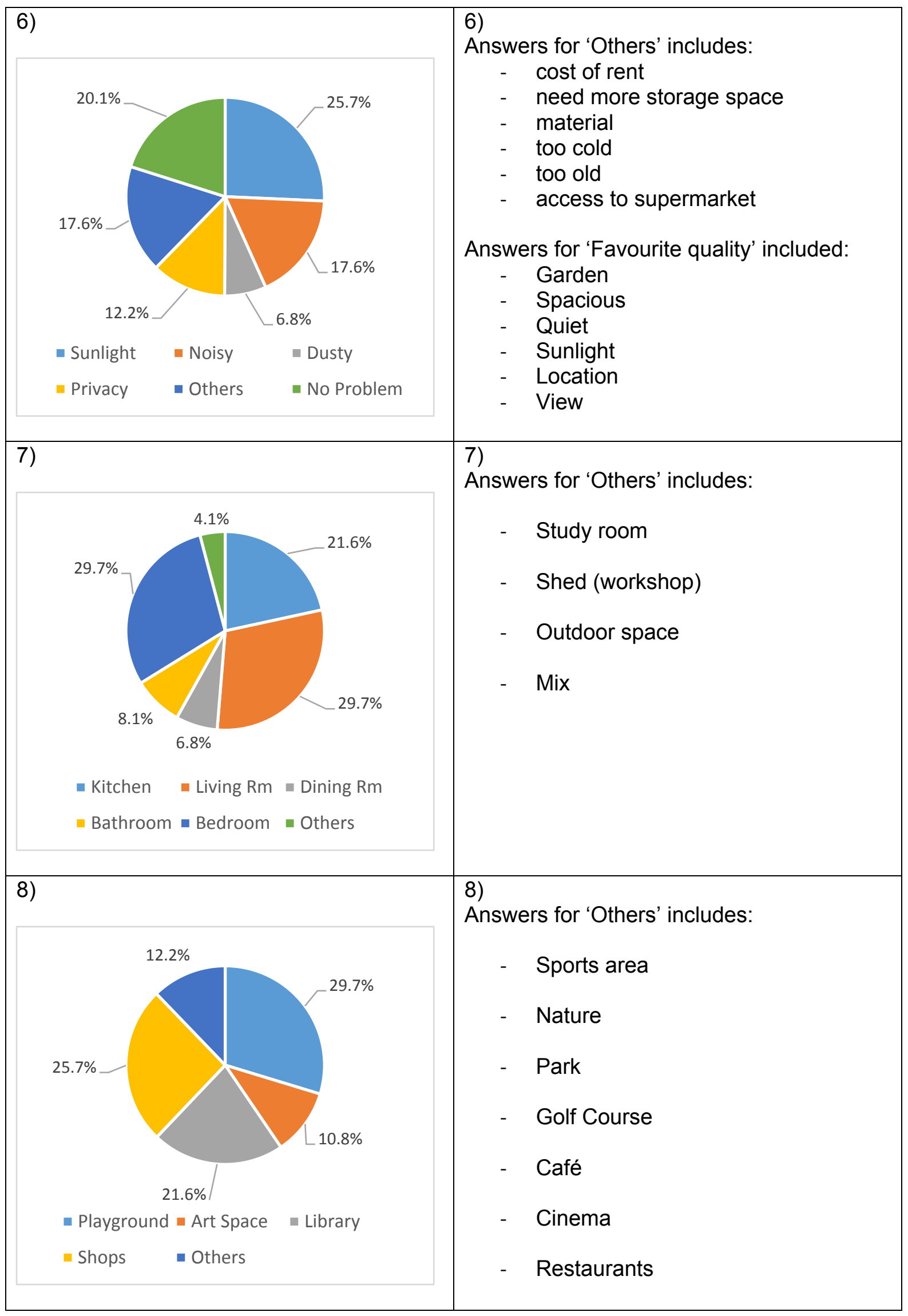




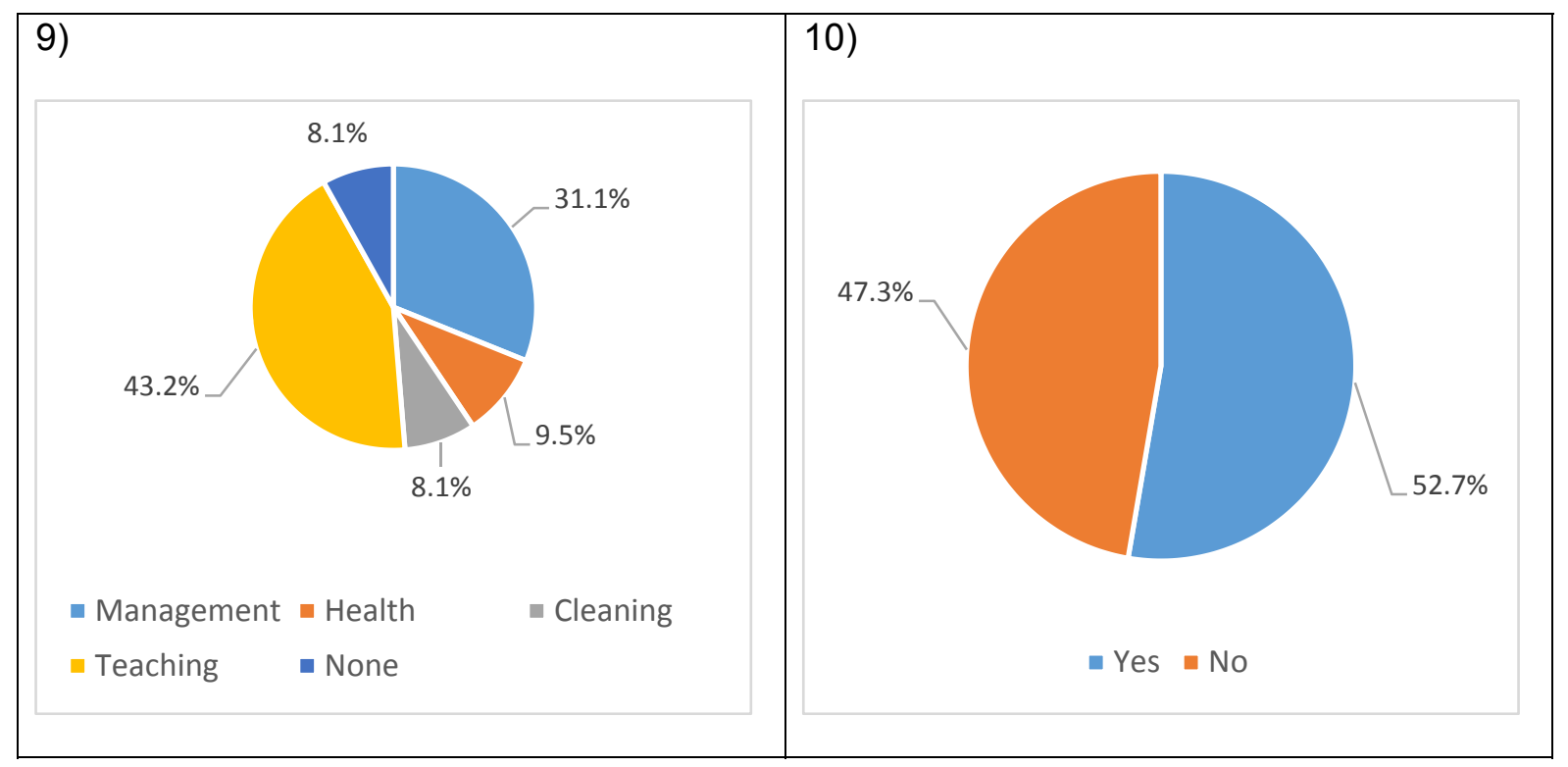


3. The 'Post' survey.

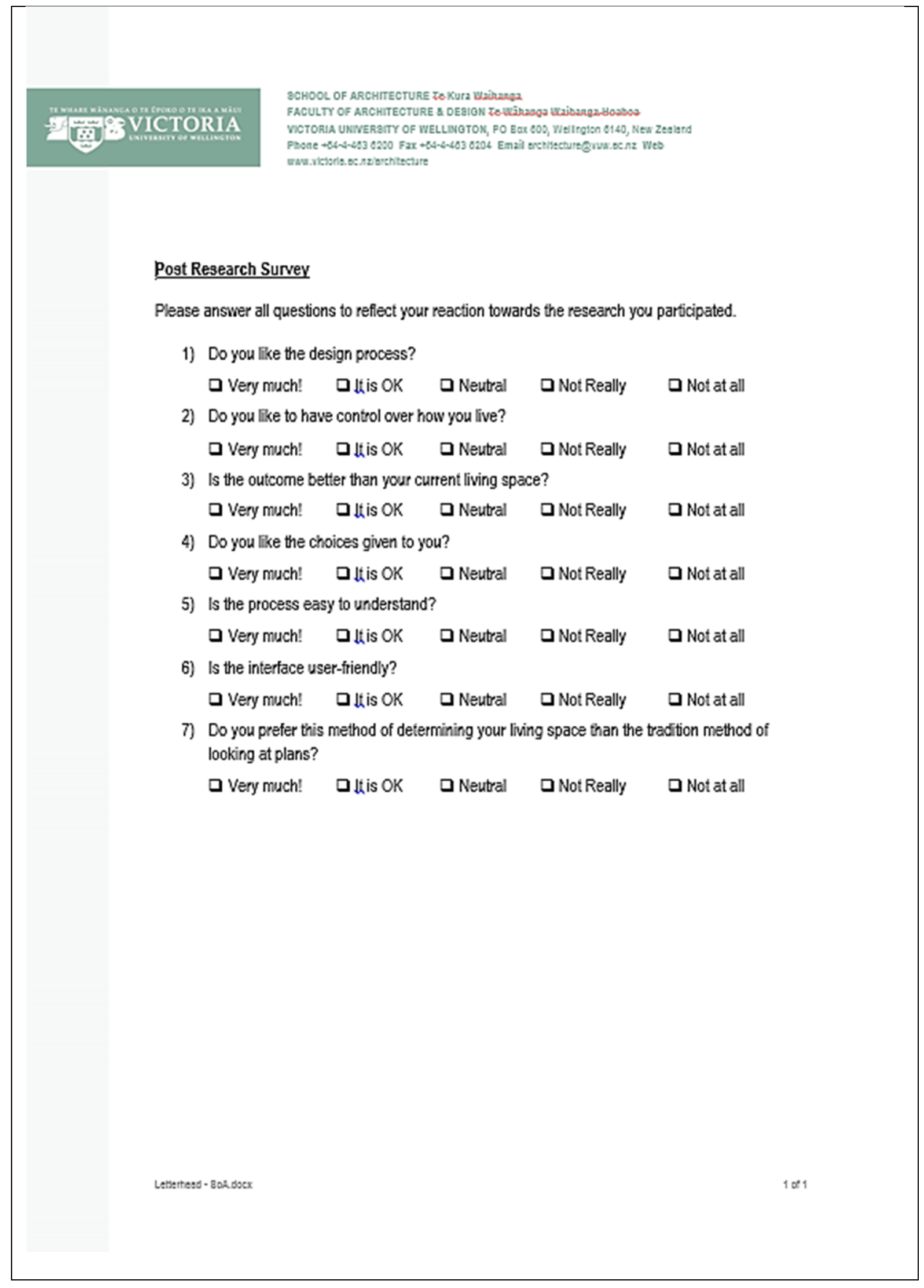


4. Results of the 'Post' survey (Total 73 participants, including 12 professionals).

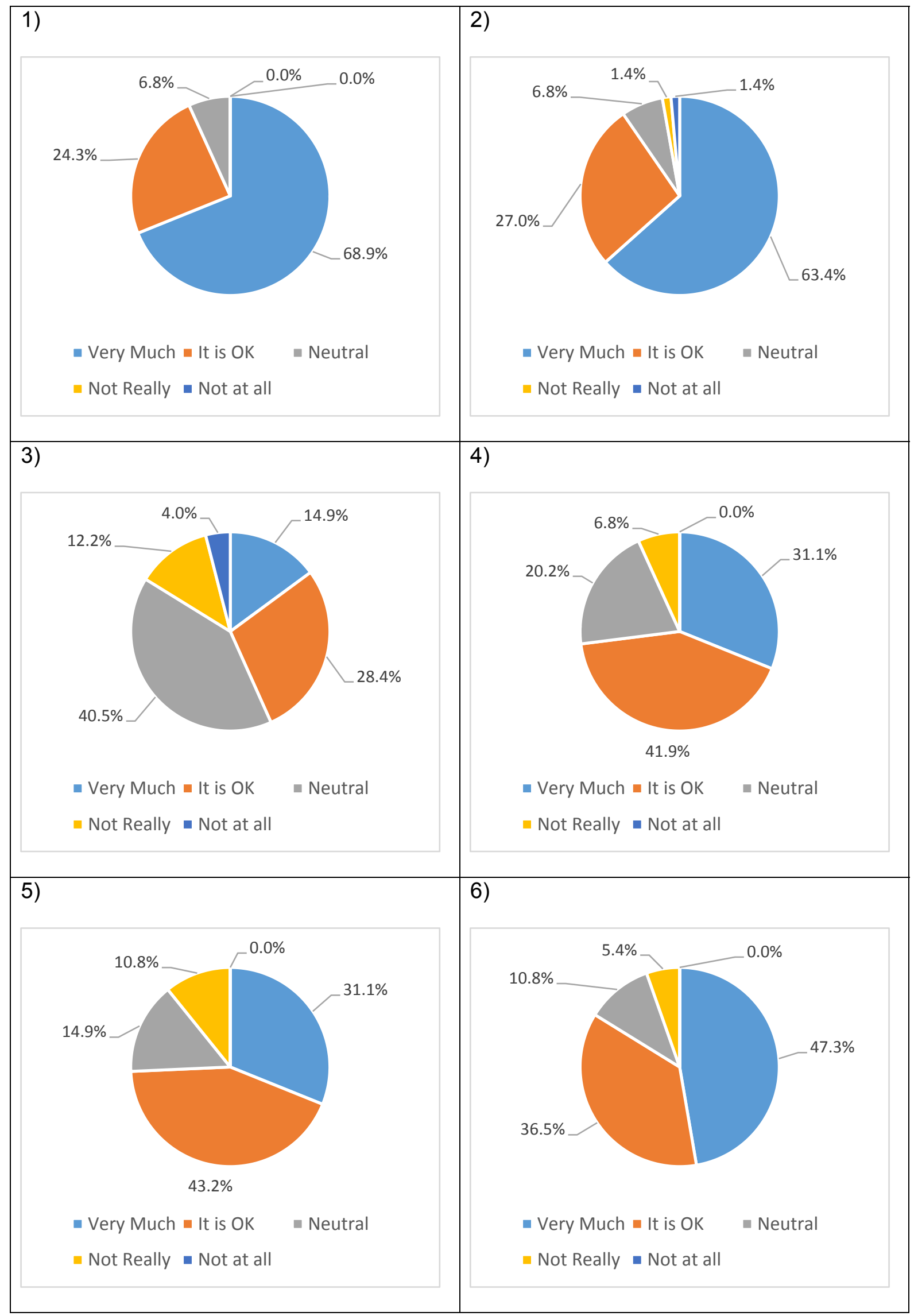




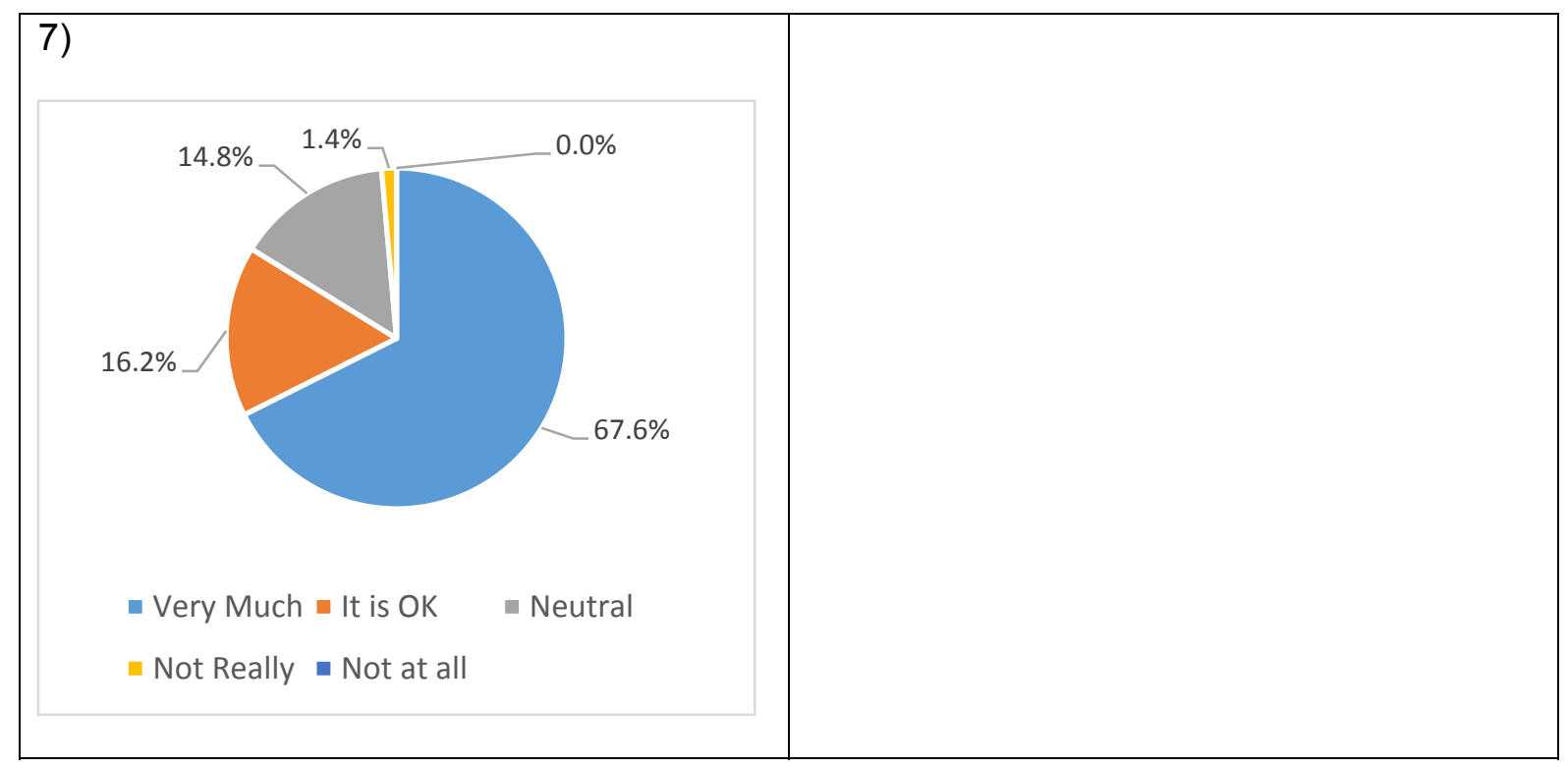




\section{Article published after the event.}

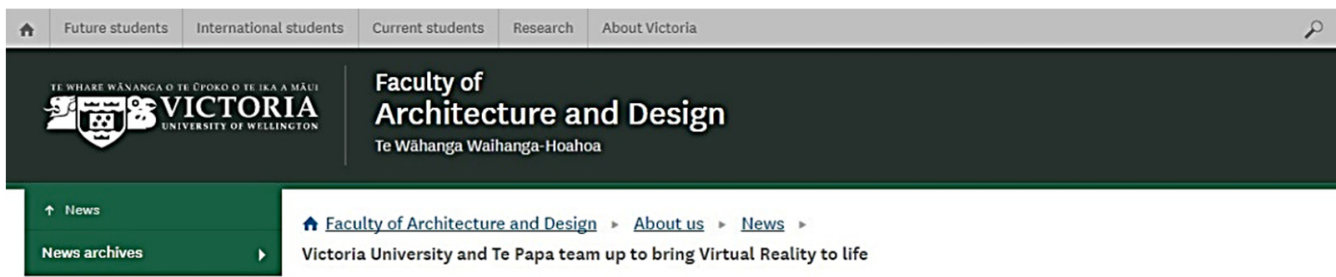

\section{Victoria University and Te Papa team up to bring Virtual Reality to life}

Victoria University of Wellington shared the latest research in virtual reality last weekend at the Te Papa Virtual Realities showcase.

28 March 2017

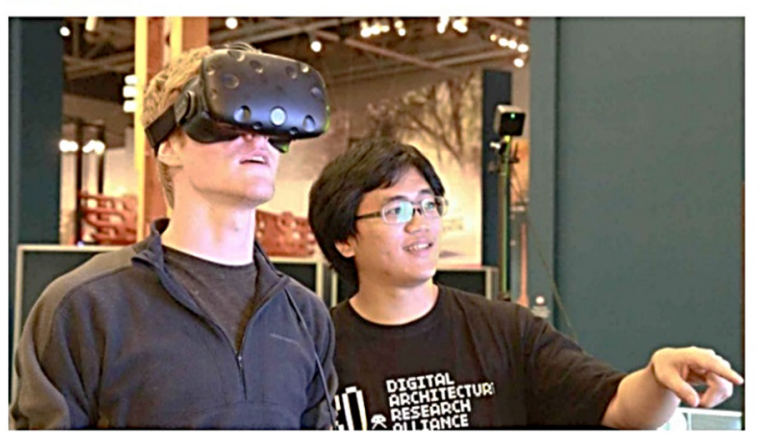

Architecture student Sky Lo talking a member of the public through what he is seeing through the VR headset

The event and exhibition ran over three days and was co-curated by Tane Moleta from the School of Architecture with colleagues from Te Papa. Demos from Victoria showcasing staff and student research came from the Schools of Architecture, Design and Engineering and Computer Science, and Associate Professor Dr Leon Gurevitch from the School of Design kicked off the weekend as MC for the sold-out Te Papa Talks' Virtual Realities

Tane explains, "the event gave people the opportunity to experience live demos, meet the creators and be actively involved in novel ways of design communication."

Two of the student projects showcased at the event included Postgraduate Architecture students Sky Lo, who showed how VR can be used to create collaborative living spaces, with the aim of creating communities through architectural design, and Serdar Aydin whose research looks at the digital heritage experience of China's westernmost city, Kashgar

This digital heritage project uses the Faculty of Architecture and Design's new Hyve-3D. Dubbed a social, immersive 3-D virtual environment, it is a wraparound screen you stand in front of which displays the images. It doesn't require headsets so numerous people can experience it at the same time and is powered by the users smartphones.

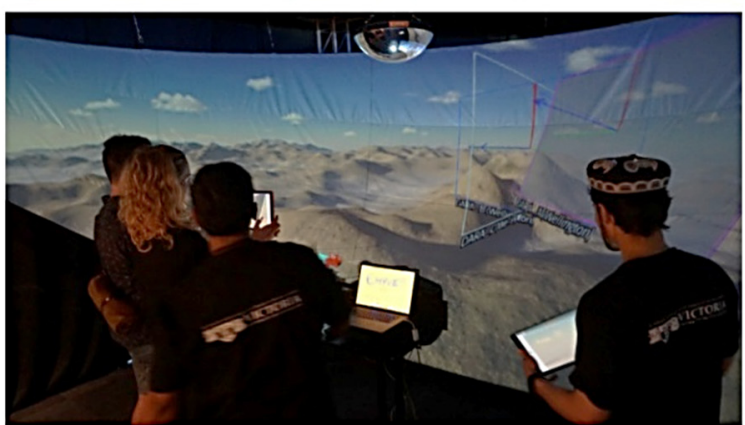

The Faculty of Arhitecture and Designs Hyve 3D

Dean of the Faculty of Architecture and Design, Professor Marc-Aurel Schnabel says, "virtual and augmented realities will become the norm for designers and architects. These technologies are already becoming mainstream in gaming and entertainment.

"Our Faculty's unique Virtual Environment Studio allows students and researchers to see how architecture can be produced and communicated seamlessly through digital platforms, and these designs can then be fed into our digital fabrication facilities using 3D printing and robotics."

Victoria is dedicated to exploring our digital future across disciplines. Researchers from various faculties are collaborating to explore and find solutions to the many challenges and opportunities associated with it. 